Wolfgang Grimme

\title{
Ordnungspolitisches Konzept der Regionalpolitik
}




\section{Wolfgang Grimme}

\section{Ordnungspolitisches Konzept der Regionalpolitik}

Institutionen bestimmen die Anpassungsfähigkeit und die Innovationsfähigkeit politischer und wirtschaftlicher Systeme und entscheiden damit mittel- und langfristig über deren Erfolg. Am Beispiel der Regionalpolitik in MecklenburgVorpommern untersucht die Arbeit die institutionellen Strukturen in Europa und Deutschland. Regionalpolitik wird durch konstruktivistische Politikkonzeptionen geprägt und im Rahmen eines verflochtenen politischen Systems geplant und implementiert. Dies führt zur Zentralisierung der Entscheidungskompetenzen, zu massivem rent-seeking-Verhalten und somit zur ineffizienten Allokation knapper Mittel. Durch eine ordnungspolitische Reform der Regionalpolitik, die Entscheidungskompetenzen radikal dezentralisiert, könnte ihre Effektivität erheblich erhöht werden.

Wolfgang Grimme wurde 1965 in Uetersen geboren. Studium der Wirtschaftsund Organisationswissenschaften an der Universität der Bundeswehr Hamburg von 1987 bis 1991 im Rahmen der Ausbildung zum Luftwaffenoffizier. Nach dem Ausscheiden aus der Bundeswehr (1994) wissenschaftlicher Mitarbeiter eines Bundestagsabgeordneten sowie freiberufliche Tätigkeit als Berater, Dozent und Trainer. Seit 1996 wissenschaftlicher Mitarbeiter von HAUS RISSEN Internationales Institut für Politik und Wirtschaft (Hamburg). Promotion 1997. 
Ordnungspolitisches Konzept der Regionalpolitik 


\title{
SCHRIFTEN ZUR WIRTSCHAFTSTHEORIE UND WIRTSCHAFTSPOLITIK
}

\author{
Herausgegeben von \\ Rolf Hasse, Wolf Schäfer, Thomas Straubhaar und Klaus W. Zimmermann
}

\section{Band 6}

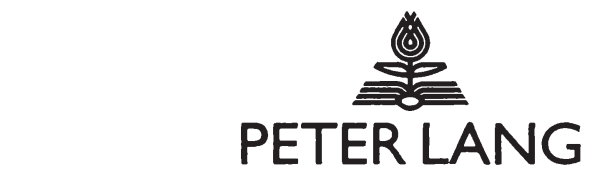

Frankfurt am Main - Berlin - Bern - New York - Paris - Wien 


\title{
Wolfgang Grimme
}

\section{Ordnungspolitisches Konzept der Regionalpolitik}

\author{
Darstellung der Defizite
}

und des Reformbedarfs der Regionalpolitik am Beispiel Mecklenburg-Vorpommerns

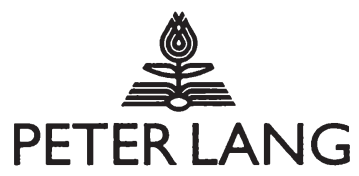

Europäischer Verlag der Wissenschaften 
Die Deutsche Bibliothek - CIP-Einheitsaufnahme

Grimme, Wolfgang:

Ordnungspolitisches Konzept der Regionalpolitik : Darstellung der Defizite und des Reformbedarfs der Regionalpolitik am Beispiel Mecklenburg-Vorpommerns / Wolfgang Grimme. Frankfurt am Main ; Berlin ; Bern ; New York ; Paris ; Wien : Lang, 1997

(Schriften zur Wirtschaftstheorie und Wirtschaftspolitik ;

Bd. 6)

Zugl.: Hamburg, Univ. der Bundeswehr, Diss., 1997

ISBN 3-631-32442-1

Open Access: The online version of this publication is published on www.peterlang.com and www.econstor.eu under the international Creative Commons License CC-BY 4.0. Learn more on how you can use and share this work: http://creativecommons. org/licenses/by/4.0.

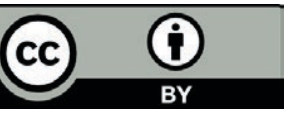

This book is available Open Access thanks to the kind support of ZBW - Leibniz-Informationszentrum Wirtschaft.

\author{
D 705 \\ ISSN 1433-1519 \\ ISBN 3-631-32442-1 \\ ISBN 978-3-631-75004-9 (eBook) \\ (c) Peter Lang $\mathrm{GmbH}$ \\ Europäischer Verlag der Wissenschaften \\ Frankfurt am Main 1997 \\ Alle Rechte vorbehalten.
}

Das Werk einschließlich aller seiner Teile ist urheberrechtlich geschützt. Jede Verwertung außerhalb der engen Grenzen des

Urheberrechtsgesetzes ist ohne Zustimmung des Verlages unzulässig und strafbar. Das gilt insbesondere für Vervielfältigungen, Übersetzungen, Mikroverfilmungen und die Einspeicherung und Verarbeitung in elektronischen Systemen.

\title{
Printed in Germany 124567
}




\section{Vorwort}

Am Anfang der Arbeit stand die unbegründete Vermutung, daß die Effektivität der wirtschaftlichen Förderprogramme für die Neuen Länder weit von dem entfernt ist, was man als optimal bezeichnen könnte. Die ersten Anstrengungen widmete ich somit dem Versuch, diese zu ermitteln. Eine adäquate theoretische Basis in Form von ökonometrischen Modellen schien vorhanden zu sein. Es zeigte sich jedoch sehr bald, daß diese Modelle nicht anwendbar sind. Es mangelt an der notwendigen Datenbasis, um die Wirksamkeit der Regionalförderung mit einer Zeitreihenanalyse zu untersuchen. Bei näherem hinsehen wurde jedoch ein viel wichtigerer Mangel deutlich. Es gab und gibt keine hinreichend operationalisierten Ziele für die vielfältigen Programme.

Dies führte zu einer anderen Fragestellung. Nicht die Effektivität der Regionalförderung stand im Mittelpunkt. Vielmehr galt es unter einer ordnungspolitischen Perspektive zu untersuchen, mit welchen institutionellen Rahmenbedingungen eine möglichst effektive Allokation knapper öffentlicher Fördermittel zu gewährleisten ist.

Den weiteren Gang der Arbeit haben viele Menschen positiv begleitet. Besonderer Dank gilt zunächst meinem Erstgutachter Prof. Dr. Rolf H. Hasse, der mit den richtigen Fragen und Hinweisen für Struktur und Stringenz meiner Argumentation sorgte. Mein Dank gilt ebenso Prof. Dr. Thomas Straubhaar, der das Zweitgutachten erstellte. Die anregenden Diskussionen mit Dr. Wolfram Högel ermöglichten mir tiefe Einblicke in das Werk von F.A. von Hayek.

Last, put not least möchte ich mich für die Geduld und die tatkräftige Unterstützung bei der Korrektur unzähliger Manuskriptseiten bei meiner Verlobten Jutta ganz herzlich bedanken. 
Wolfgang Grimme - 978-3-631-75004-9

Downloaded from PubFactory at 01/11/2019 09:23:21AM

via free access 
Inhaltsverzeichnis

$\begin{array}{ll}\text { Problemstellung } & 13\end{array}$

I. Theoretische Fundierung der Regionalpolitik und Zuord- 17 nung regionalpolitischer Kompetenzen im föderalen Staat

1. Regionalpolitik: Definition und Einordnung des Gegenstan- 18 des

$1.1 \quad$ Regionalpolitik und wirtschaftspolitische Konzepte 18

1.2 Regionalpolitik als öffentliches Gut 20

1.3 Zusammenfassung 22

2. Theoretische Fundierung der Regionalpolitik und unterneh- 23 merischer Standortentscheidungen

$2.1 \quad$ Die theoretische Fundierung der Regionalpolitik 26

2.1.1 Theorie der zentralen Orte 26

$\begin{array}{ll}2.1 .2 & \text { Die Exportbasistheorie } \\ 2.3 & 27\end{array}$

2.1.3 Die Konzeption der Wachstumspole 29

2.1.4 Neue Wachstumsmodelle 31

2.2 Die unternehmerische Standortentscheidung 33

$\begin{array}{lll}2.3 & \text { Zusammenfassung } & 34\end{array}$

3. Zuordnung regionalpolitischer Entscheidungs- und Finanzie- 36 rungskompetenzen im föderalen Staat

3.1 Zentrale versus dezentrale Regionalpolitik 36

3.2 Ordnungspolitische Regionalpolitik 40

3.2.1 Wettbewerb als Entdeckungsverfahren 41

3.2.2 Wettbewerb zwischen regionalen Einheiten - ein ordnungspoliti- 44 sches Konzept der Regionalpolitik

$\begin{array}{lll}3.3 & \text { Zusammenfassung } & 47\end{array}$

II. Regionalpolitik in Mecklenburg-Vorpommern 49

4. Wirtschaftsstruktur Mecklenburg-Vorpommerns 50

5. Regionalpolitik in Mecklenburg-Vorpommern: Die Ziele 54

5.1 Grundlegende regionalpolitische Ziele in Verfassungen und Ge- 55 setzen

5.1.1 Zielvorgaben des Grundgesetzes und der Landesverfassung 55

5.1.2 Das Raumordnungsgesetz 56

5.1.3 Raumordnung in Mecklenburg-Vorpommern 58 
5.1.4 Prozeß der Landesplanung: Top-down statt bottom-up 61

5.2 Ziele der regionalen Wirtschaftspolitik 62

5.2.1 Regionalpolitik der EG: Ziele 63

5.2.2 Ziele der Gemeinschaftsaufgabe 66

5.2.3 Regional- und Wirtschaftspolitische Ziele Mecklenburg- 67 Vorpommerns

$\begin{array}{lll}\text { 5.2.3.1 Allgemeine wirtschaftspolitische Ziele } & 67\end{array}$

5.2.3.2 Ziele der Regionalförderung in Mecklenburg-Vorpommern 69

5.3 Zusammenfassung: Das Zielsystem der Regionalpolitik für 71 Mecklenburg-Vorpommern

6. Regionalpolitik in Mecklenburg-Vorpommern: Die Instru- 73 mente Gemeinschaftsaufgabe und EFRE

$\begin{array}{lll}6.1 & \text { Die Gemeinschaftsaufgabe } & 74\end{array}$

$\begin{array}{lll}\text { 6.1.1 Institutionelle Rahmenbedingungen } & 74\end{array}$

$\begin{array}{lll}\text { 6.1.2 Inhaltliche Vorgaben der GA } & 78\end{array}$

6.2 Einsatz des EFRE in Mecklenburg-Vorpommern 83

6.2.1 Institutionelle Rahmenbedingungen der EG Strukturpolitik 83

6.2.2 Inhaltliche Vorgaben der EG Strukturpolitik 87

$\begin{array}{lll}6.3 & \text { Zusammenfassung } & 90\end{array}$

7. Regionalpolitik in Mecklenburg-Vorpommern: Implementa- 91 tion der Gemeinschaftsaufgabe und des EFRE

7.1. Implementation der Regionalpolitik: Programme, Verfahren, 92 Mittel

7.1.1 Regionalförderprogramme für Mecklenburg-Vorpommern 92

7.1.2 Bewilligungsverfahren 96

7.1.3 Mittelausstattung der Regionalförderung in Mecklenburg- 98 Vorpommern

7.2 Einsatz der Fördermittel 101

7.2.1 Förderung im Rahmen der GA: Ein Überblick 102

$\begin{array}{lll}\text { 7.2.2 Schwerpunkte der Regionalförderung } & 109\end{array}$

$\begin{array}{lll}\text { 7.2.3 Förderung der zentralen Orte } & 114\end{array}$

7.3 Umsetzung der regionalpolitischen Ziele und Programme 116

$\begin{array}{ll}7.4 & 120\end{array}$

8. Wirtschaftspolitik und Wirtschaftsförderung des Landes 122 Mecklenburg-Vorpommern

8.1 Handlungsfreiräume für eine eigenständige Wirtschaftspolitik des 122 Landes

8.1.1 Das Land als Träger der regionalen Wirtschaftspolitik 
8.1.2 Finanzielle Handlungsspielräume des Landes Mecklenburg- 128 Vorpommern

8.2 Wirtschaftspolitik und Wirtschaftsförderung des Landes Meck- 134 lenburg-Vorpommern

8.2.1 Handlungsfelder der Wirtschaftspolitik und der Wirt- 134 schaftsförderung des Landes Mecklenburg-Vorpommern

$\begin{array}{ll}\text { 8.2.2 Wirtschaftsförderung des Landes } & 136\end{array}$

$\begin{array}{lll}\text { 8.2.2.1 Mittel der Landesförderung } & 136\end{array}$

8.2.2.2 Die Instrumente der Wirtschaftsförderung 138

$\begin{array}{lll}8.3 & \text { Zusammenfassung } & 149\end{array}$

III. Reform, Entwicklung und Reformfähigkeit der regionalpoli- 153 tischen Institutionen

9. Notwendige Reformen zur Einführung einer ordnungspoliti- 154 schen Regionalpolitik

9.1 Allgemeine Prinzipien einer ordnungspolitischen Regionalpolitik 154

9.1.1 Ordnungspolitische Regionalpolitik in der Gemeinschaft und in 158 Deutschland

9.2 Entwicklung der regionalpolitischen Institutionen 161

9.2.1 Die Reform der EG Strukturfonds 162

9.2.2 Entwicklung der Regionalpolitik im Rahmen der GA 164

9.3 Ursachen der Reformblockade 166

$\begin{array}{lll}9.4 & \text { Zusammenfassung } & 172\end{array}$

10. Zusammenfassung der Arbeit 175

$\begin{array}{ll}\text { Literaturverzeichnis } & 181\end{array}$ 


\section{Verzeichnis der Tabellen und Abbildungen}

Tabelle 1 Strukturunterschiede in Ostdeutschland 51

Tabelle 2 Beschäftigtenanteile nach Wirtschaftssektoren in Meck- 52 lenburg-Vorpommern und Schleswig-Holstein 1990

Tabelle 3 Beschäftigtenabbau ausgewählter Zweige des verarbeiten- 53 den Gewerbes

Tabelle $4 \quad$ Umsätze des verarbeitenden Gewerbes 54

$\begin{array}{lll}\text { Abbildung } 1 \text { Regionalpolitische Ziele in Mecklenburg-Vorpommern } & 72\end{array}$

Abbildung 2 Wirkungsschema der Politikverflechtung 86

Abbildung 3 Regionalförderprogramme Mecklenburg-Vorpommern 93 1991-1994

Abbildung 4 Fördergebietskulisse in Mecklenburg-Vorpommern 95

Tabelle 5 Bearbeitungsdauer von Förderanträgen für Mittel der GA 97 in Mecklenburg-Vorpommern von 1990-1994

Tabelle 6 Mittelansatz Gemeinschaftsaufgabe, Aufschwung Ost und 99 EFRE in Mecklenburg-Vorpommern

Tabelle 7 Zuweisungen von Bund und EG für MVP: Mittelansatz 100 des Haushaltes

Tabelle 8 Förderung der gewerblichen Wirtschaft 01.10 .1990 - 103 30.06.1994 in Mecklenburg-Vorpommern

Tabelle 9 Förderung der wirtschaftsnahen Infrastruktur vom 105 01.10.90 bis 30.06.1994 in Mecklenburg-Vorpommern

Tabelle 10 Gewerbeflächenbestand in Mecklenburg-Vorpommern 107 1992

Tabelle 11 Förderintensität in Mecklenburg-Vorpommern vom 111/ 01.10 .1990 - 30.06.1994

Tabelle 12 Förderung von OZ, MZ und MZTF in Mecklenburg- 115 Vorpommern vom 01.10.1990 - 30.06.1994

Tabelle 13 Bedeutung der Wirtschaftsförderung des Landes Mecklen- 129 burg-Vorpommern

Tabelle 14 Bedeutung von Zweckzuweisungen im Etat des Wirt- 131 schaftsministeriums

Tabelle 15 Fiskalische Kosten der Förderung privater Investitionen in 133 den neuen Bundesländern

Tabelle 16 Landesprogramme in Mecklenburg-Vorpommern

Abbildung 5 Landeseigene Programme der Wirtschaftsförderung in 139 Mecklenburg-Vorpommern 1994 


\section{Abkürzungsverzeichnis}

Abl (EG) Amtsblatt der Europäischen Gemeinschaft

AO Aufschwung Ost

BGB1 Bundesgesetzblatt

BMWi Bundesministerium für Wirtschaft

EFRE Europäischer Fonds für Regionale Entwicklung

ESF Europäischer Sozialfonds

EUGH Europäischer Gerichtshof

EWGV Europäische Wirtschaftsgemeinschaft - Vertrag

FAZ Frankfurter Allgemeine Zeitung

GA Gemeinschaftsaufgabe zur Verbesserung der regionalen Wirtschaftsstruktur

GI Gemeinschaftsinitiative

IWH Institut für Wirtschaftsforschung Halle

KMU Klein- und mittelständische Unternehmen

L Landkreis

LPlg Landplanungsgesetz

MVP Mecklenburg-Vorpommern

MZ Mittelzentrum

MZTF Mittelzentrum mit Teilfunktion

NBB Neubrandenburg

ÖPNV Öffentlicher Personennahverkehr

o.V. ohne Verfasser

$\mathrm{OZ} \quad$ Oberzentrum

ROG Raumordnungsgesetz

SPNV Schienenpersonennahverkehr

ST Stadt

THA Treuhandanstalt

WD Wirtschaftsdienst 
Wolfgang Grimme - 978-3-631-75004-9

Downloaded from PubFactory at 01/11/2019 09:23:21AM

via free access 


\section{Problemstellung}

Ökonomisch wie politisch leben wir in einer Zeit des durchgreifenden Wandels. Das letzte Jahrzehnt des ausgehenden Jahrtausends ist geprägt durch die Begriffe „Globalisierung“ und „Multipolarität". Politische und insbesondere wirtschaftspolitische Akteure erkennen zunehmend, daß die alten Konzepte und Programme den neuen Herausforderungen nicht mehr gerecht werden. Dies ist historisch gesehen keine neuartige Situation, denn Zeiten rasanten Wandels, die politische und ökonomische Systeme verändert haben, hat es immer wieder gegeben. Der Erfolg politischer und wirtschaftspolitischer Systeme hängt daher mittel- und langfristig von der Fähigkeit ab, auf Veränderungen der Rahmendaten mit adäquaten Systemveränderungen zu reagieren. Die politische und wirtschaftspolitische Innovationsfähigkeit eines Staates ist in dieser Situation der wichtigste Erfolgsfaktor, da die Gesellschaft, ,... that permits the maximum generation of trials will be most likely to solve problems through time."' Die Handlungs- und Innovationsfähigkeit eines Systems wird durch die das System begründenden Institutionen bestimmt. Institutionen sind im Sinne der modernen Institutionenökonomie alle formalen und informalen Regelungen, die menschliches Verhalten bestimmen. D. North führt hierzu aus: "Institutions are the rule of the game in a society or, more formally, are the humanly devised constraints that shape human interaction. ... Institutions define and limit the set of choices of individuals." ${ }^{2}$ Die Leistungsfähigkeit eines institutionellen Rahmens zeigt sich somit immer dann besonders deutlich, wenn auf Grund signifikanter Datenänderungen Anpassungen notwendig werden. Die aktuellen politischen und wirtschaftspolitischen Herausforderungen stellen derzeit die institutionelle Struktur Deutschlands und Europas auf die Probe. Im Kern aller wirtschaftspolitischen Herausforderungen steht die Frage nach der Leistungsfähigkeit der vorhandenen Institutionen. Da es nicht möglich ist, im Rahmen dieser Arbeit, eine Totalanalyse der institutionellen Strukturen vorzunehmen, beschränke ich mich exemplarisch auf einen Teilbereich, in dem der Anpassungsbedarf besonders signifikant ist. Gegenstand der Untersuchung ist daher die Regional- und Strukturpolitik, die im Rahmen eines verflochtenen Systems von der EG, dem Bund und den Ländern getragen und gestaltet wird. Sowohl in der Gemeinschaft als auch in Deutschland dominieren in diesem Bereich quantitativ interventionistische Politikkonzeptionen. Diese wurden im Zuge der deutschen Einheit mit geringfügigen Änderungen auf die neuen Bundesländer übertragen. Zentrales Instrument der ersten vier Aufbaujahre war die Gemeinschaftsaufgabe zur Verbesserung der regionalen Wirtschaftsstruktur (GA), die in Deutschland seit 1969 eingesetzt wird.

D.C. North: "Institutions, Institutional Change and economic performance", 1990, S. 3. 
Die europäische und deutsche Regionalpolitik steht vor großen Herausforderungen. Für ein Gelingen der Währungsunion ist ein Mindestmaß an realer Konvergenz eine notwendige Bedingung. Ferner will Europa die mittel- und osteuropäischen Staaten in seine Strukturen integrieren. Auch hierfür ist ein Mindestmaß an realer Konvergenz eine notwendige Bedingung. Die Aufgabe deutscher Regionalpolitik ist es, der politischen Zielvorgabe der Einheitlichkeit der Lebensverhältnisse folgend, die Disparitäten in der wirtschaftlichen Leistungsfähigkeit zwischen Ost- und Westdeutschland abzubauen. Regionalpolitik hat also für die deutsche Einheit, die europäische Integration sowie im globalen Standortwettbewerb eine herausragende ökonomische und politische Bedeutung. Falsche Politikkonzeptionen, unzureichende institutionelle Strukturen und die daraus resultierende ineffiziente Allokation knapper Mittel können das Gelingen der Währungsunion, der Integration Mittel- und Osteuropas und die ökonomische Stabilisierung der deutschen Einheit gefährden. Aus ökonomischer Sicht gilt es, die für diese Herausforderungen richtigen wirtschaftspolitischen Konzeptionen zu definieren.

In der Regionalpolitik geht es immer auch um die Verteilung bzw. Umverteilung von finanziellen Ressourcen zugunsten bestimmter Regionen. Da dies so ist, sind regionalpolitische Entscheidungen in hohem Maße politik-ökonomischen Rationalitäten unterworfen. Regionalpolitik hat daher ihren hohen politischen Stellenwert, nicht zuletzt weil sie im Sinne der Nutzenmaximierung der politischen Akteure eingesetzt werden kann.

Sie befindet sich damit ganz besonders im Spannungsverhältnis zwischen ökonomischer und politischer Rationalität. Dieser Umstand dürfte eine der wesentlichen Ursachen für die nur sehr geringen Erfolge der deutschen und europäischen Regionalpolitik sein. Im Rahmen der Arbeit sollen folgende Thesen geprüft werden:

a. Die deutsche und europäische Regionalpolitik ist durch konstruktivistische wirtschaftspolitische Konzeptionen geprägt. Hierin liegt ihr primäres Defizit und die Ursache für die geringe Effektivität.

b. Die aus den bestehenden institutionellen Rahmenbedingungen resultierende Kompetenzzuweisung führt - im Sinne der regionalen Präferenzen und der fiskalischen Äquivalenz - zu einer ineffizienten Allokation regionalpolitischer Mittel.

c. Eine Reform muß sich an dem Leitbild einer ordnungspolitischen Regionalpolitik orientieren. Hierfür sind grundlegende Änderungen der institutionellen Strukturen notwendig.

d. Die vorhandenen politik-ökonomischen Rationalitäten stehen einer durchgreifenden Reform der institutionellen Strukturen im Wege. Es sind lediglich partielle Veränderungen realisierbar. 
Der Gang der Untersuchung gliedert sich daher wie folgt. Im ersten Abschnitt wird die theoretische Grundlage für die empirische Analyse gelegt. Der Untersuchungsgegenstand wird eingegrenzt (Kapitel 1), die die Regionalpolitik fundierenden regionalwirtschaftlichen Theorien werden analysiert (Kapitel 2), und es werden Kriterien für die Zuordnung der regionalpolitischen Kompetenzen dargestellt (Kapitel 3). Ferner wird, basierend auf dem Hayekschen Wettbewerbsverständnis, ein ordnungspolitisches Konzept der Regionalpolitik entworfen, das Grundlage für die weiteren Analysen ist (Kapitel 3).

Basierend auf diesen theoretischen Überlegungen werden im zweiten Abschnitt der Arbeit die institutionellen Strukturen, in denen Regional- und Strukturpolitik definiert und implementiert wird, am Beispiel Mecklenburg-Vorpommerns untersucht. Der empirische Teil der Untersuchung bezieht sich exemplarisch auf die Regional- und Strukturpolitik in Mecklenburg-Vorpommern vom 03.10.1990 bis zum 30.06.1994. Die Einschränkung des Zeitraumes ist notwendig, da eine Querschnittsanalyse der vorhandenen Daten aufgrund der Kreisgebietsreform, die ab 01.07.94 in Kraft trat, nur für diesem Zeitraum möglich ist. Die Gliederung des Abschnitts orientiert sich an den Phasen eines idealtypischen Planungsprozesses. Die Darstellung der Ausgangslage (Kapitel 4), d.h. eine Analyse der Wirtschaftsstruktur Mecklenburg-Vorpommerns, bildet die Basis für den Planungsprozeß. Anschließend werden die regionalpolitischen Ziele (Kapitel 5) untersucht. Es gilt zu prüfen, ob der Regionalpolitik ein konsistentes Zielsystem zugrunde liegt. Die Umsetzung der Ziele erfolgt mittels regionalpolitischer Programme (Kapitel 6), die auf einer oder mehreren regionalwirtschaftlichen Theorien und einem wirtschaftspolitischen Konzept basieren. Das Kapitel konzentriert sich auf die institutionelle Struktur, in der Regionalpolitik geplant und implementiert wird, und prüft, ob ordnungspolitische Ansätze erkennbar sind. Den Abschluß eines Planungsprozesses bildet die Evaluation der Maßnahmen und Programme (Kapitel 7). Aufgrund fehlender Daten und der Kreisgebietsreform in MecklenburgVorpommern ist keine Wirkungsanalyse im engeren Sinne möglich. Mit einer Querschnittsanalyse kann jedoch geprüft werden, ob die Implementation der Programme, an den Zielvorgaben orientiert, erfolgt ist. Da eine ordnungspolitische Regionalpolitik auf den konkreten Maßnahmen und Programmen vornehmlich der Landesebene beruht, gilt es zu untersuchen, ob und wenn ja, welche Aktivitäten das Land Mecklenburg-Vorpommern neben der Gemeinschaftsaufgabe (Gemeinschaftsaufgabe zur Verbesserung der regionalen Wirtschaftsstruktur, kurz: GA) und dem Europäischen Fonds für regionale Entwicklung (EFRE) entwickelt (Kapitel 8). Hierzu werden zunächst die derzeit vorhanden potentiellen Handlungsfreiräume und deren finanzielle Absicherung betrachtet.

Im dritten Abschnitt werden, basierend auf der empirischen Analyse, die notwendigen Reformen für eine ordnungspolitische Regionalpolitik herausgearbeitet und mit den nach 1994 tatsächlich durchgeführten Veränderungen der GA und des EFRE verglichen (Kapitel 9). Die Gründe für die erwartbaren Reformdefizite 
werden analysiert, und es werden die im Rahmen der bestehenden politikökonomischen Rationalitäten möglichen Reformen herausgearbeitet. Das abschließende Kapitel 10 faßt die wesentlichen Ergebnisse der Arbeit zusammen. 


\section{Theoretische Fundierung der Regionalpolitik und Zuordnung regional- politischer Kompetenzen im föderalen Staat}

Im ersten Abschnitt der Arbeit soll die theoretische Basis für die empirische Analyse der Regionalpolitik in Mecklenburg-Vorpommern gelegt werden. Zunächst gilt es, den Untersuchungsgegenstand näher einzugrenzen. Es gibt keine einheitliche Definition für Regionalpolitik und auch kein einheitliches wirtschaftspolitisches Konzept für regionalpolitische Ansätze und Programme. Den meisten regionalpolitischen Konzepten liegt die Vorstellung zugrunde, daß Regionalpolitik ein öffentliches Gut sei. Die Bandbreite der durch die Regionalpolitik bereitgestellten Güter reicht von der Infrastrukturförderung bis zur direkten Unternehmenssubvention, eine Spannweite die Zweifel aufkommen läßt, ob die pauschale Interpretation der Regionalpolitik als öffentliches Gut gerechtfertigt ist. Diese Fragen werden im ersten Kapitel behandelt.

Die in Deutschland und Europa praktizierte Regionalpolitik erhebt den Anspruch, Einfluß auf die wirtschaftliche Entwicklung in bestimmten Regionen nehmen zu können. Dieser Anspruch ist nur dann glaubhaft, wenn ihr eine Theorie zugrunde liegt, die die wirtschaftliche Entwicklung im Raum erklärt. Eine bewußte und geplante Entwicklung ist nur dann möglich, wenn die Faktoren bekannt sind, die eine wirtschaftliche Entwicklung in einer Region determinieren. Im zweiten Kapitel werden die verschiedenen Regionaltheorieen analysiert, die für die Begründung der deutschen bzw. europäischen Regionalpolitik herangezogen werden. Es wird geprüft, ob diese Theorien eine Basis für regionalpolitische Ansätze und Programme sein können. Ferner wird analysiert, welche wirtschaftspolitische Konzeption einer Regionalpolitik zugrunde liegt, die auf eine bestimmte Theorie fußt.

Regionalpolitik findet statt. Sie wird im Rahmen der föderalen europäischen Struktur geplant und implementiert. Damit stellt sich die Frage, auf welcher Ebene dieses föderalen Systems die Handlungs- und Finanzierungskompetenz für Regionalpolitik angesiedelt werden sollte. Hierzu werden zwei Ansätze gewählt. Zum einen wird grundsätzlich gefragt, ob eine öffentliche Leistung zentral oder dezentral angeboten werden sollte. Diese statische Analyse wird ergänzt durch eine dynamische Analyse. Auch öffentliche Leistungen sind einem Wettbewerb ausgesetzt, Art, Qualität und Struktur müssen sich veränderten Rahmenbedingungen und Anforderungen anpassen. Basierend auf dem dynamischen Wettbewerbskonzept Hayeks wird ein ordnungspolitisches Konzept der Regionalpolitik entwickelt, dessen wesentliches Merkmal ein regelgebundener Wettbewerb der Regionen ist. Damit dieser Wettbewerb möglich ist, muß die Kompetenzzuordnung in einer bestimmten Weise erfolgen. 


\section{Regionalpolitik: Definition und Einordnung des Gegenstandes}

In diesem ersten Kapitel müssen zunächst drei grundlegende Fragen geklärt werden.

a. Zunächst grenze ich den Begriff Regionalpolitik ein, der im Rahmen dieser Arbeit Anwendung findet.

b. Um regionalpolitische Aktivitäten einordnen zu können, müssen die ihnen zugrunde liegenden wirtschaftspolitischen Konzeptionen betrachtet werden. Ich werde drei Konzepte vorstellen, die sich durch den Grad der angenommenen Plan- und Steuerbarkeit wirtschaftlicher Prozesse unterscheiden.

c. Abschließend wird die Frage diskutiert, ob bzw. bis zu welchem Grad Regionalpolitik ein öffentliches Gut ist. Da öffentliche Güter nicht über den Markt bereitgestellt werden können, findet sich hier eine Legitimation für staatliches Handeln.

\subsection{Regionalpolitik und wirtschaftspolitische Konzepte}

Was ist Regionalpolitik eigentlich? Vor der Analyse der Regionalpolitik muß versucht werden, den Gegenstand der Untersuchung abzugrenzen. Der scheinbar klare Begriff entzieht sich bei genauerem Hinsehen einer konkreten Definition. Regionalpolitik ist ein Teilbereich der Gesamtpolitik. Sie umfaßt "... alle Bestrebungen, Handlungen und Maßnahmen, die darauf abzielen, den Ablauf des Wirtschaftsgeschehens in einem Gebiet oder Bereich zu ordnen, zu beeinflussen oder unmittelbar festzulegen. ${ }^{3}$ Unter diesen weiten Begriff fällt nicht nur die regionale Wirtschafts- und Strukturpolitik, sondern er umfaßt alle infrastrukturpolitischen und arbeitsmarktpolitischen Maßnahmen, die zur Realisierung regionaler Ziele eingesetzt werden. Andere Autoren sprechen von Raumwirtschaftspolitik. Dies ist „,... die Summe aller Bestrebungen, Maßnahmen und Handlungen, welche die Stimulierung und Lenkung von Entwicklungen in der räumlichen Verteilung ökonomischer Aktivitäten verfolgen." ${ }^{4}$

Unter beide Begriffe lassen sich alle politischen Maßnahmen subsumieren, die Auswirkungen auf die wirtschaftliche Situation einer Region haben. Neben der Infrastrukturpolitik und der eigentlichen Wirtschaftspolitik fällt unter diese Definition auch die Bildungspolitik, die Forschungspolitik, die Haushaltspolitik und die Umweltpolitik. Da der empirische Teil der Arbeit auf der Ebene Bundesland durchgeführt wird, müßte, ausgehend von dieser Definition, fast die gesamte Landespolitik betrachtet werden. Bezogen auf ein Bundesland wäre die Regionalpoli-

Definition von Herbert Giersch, zitiert nach Bernhard Oswald: "Erfolgskontrolle in der Regionalpolitik", 1980, S. 20.

Harald Jürgensen: „Raumwirtschaft II: Politik“, in Handwörterbuch der Wirtschaftswissenschaft, 1980, S. 429. 
tik nicht Teil der Wirtschaftspolitik, sondern umgekehrt. Die Analyse muß sich daher auf einen Teilbereich der regionalpolitischen Aktivitäten konzentrieren. Eine trennscharfe Abgrenzung läßt sich am besten an Hand der eingesetzten Instrumente bzw. Programme durchfuihren. Daher konzentriert sich die Arbeit auf der europäischen Ebene auf die Programme des EFRE (Europäischer Fonds für regionale Entwicklung) und auf der Bundesebene auf die GA (Gemeinschaftsaufgabe zur Verbesserung der regionalen Wirtschaftsstruktur). Ferner werden gleichartige Programme des Landes berücksichtigt. Diese Programme umfassen u.a. Maßnahmen aus den Bereichen Infrastrukturpolitik, Ausbildungspolitik, Umweltpolitik und Forschungspolitik.

Um regionalpolitische Ansätze und Programme miteinander vergleichen zu können, muß eine weitere Ebene in die Diskussion eingeführt werden. Eine Unterscheidung an Hand der Programme bzw. Programmgegenstände ist nicht sehr ergiebig. Aus ordnungspolitischer Sicht ist das den regionalpolitischen Ansätzen und Programmen zugrundeliegende wirtschaftspolitische Konzept relevant. Basierend auf diesen Konzepten können regionalpolitische Ansätze eingeordnet und bewertet werden. Ich stelle drei Konzepte vor. Auf einem Kontinuum zwischen Planbarkeit und Nichtplanbarkeit regionaler wirtschaftlicher Entwicklung werde ich auf die beiden Extremfälle und einen mittleren Ansatz eingehen.

\section{Marktversagen und Planbarkeit}

Das konstruktivistische regionalpolitische Konzept geht von der Plan- und Steuerbarkeit wirtschaftlicher Prozesse aus. Die Marktkräfte tendieren demnach zu einer ineffizienten räumlichen Allokation der Faktoren. Dieses Marktversagen muß durch politische Programme korrigiert werden. Die Raumordnungs- und Regionalpolitik soll "... den sich jeden Moment vollziehenden Entwicklungsprozeß einer Volkswirtschaft in räumlicher und in sachlicher Hinsicht ..." Aufgabe der Regionalpolitik ist die Beeinflussung der Verteilung der verschiedenen Aktivitäten auf der zur Verfügung stehenden Fläche. "Es geht also darum, jedem Teilgebiet des Staates oder des gerade betrachteten Raumes die Funktion zuzuweisen, die es im Gesamtinteresse wahrnehmen sollte." ${ }^{6}$ Der Konzeption der Regionalpolitik liegt somit die Annahme des "benovelent despotism that will implement the efficiency criteria" ${ }^{7}$ zugrunde.

5 Rainer Thoss: "Ermittlung der konkurrierenden Regionen - Interregionale und intraregionale Konkurrenzbeziehungen", in "Konkurrenz zwischen kleinen Regionen", Nomos, 1978, S. 41 .

6 Rainer Thoss: "Ermittlung der konkurrierenden Regionen - Interregionale und intraregionale Konkurrenzbeziehungen", a.a.O., S. 48.

7 J.M. Buchanan: „The Power to Tax. Analytical Foundations of a Fiscal Constitution“, Cambridge University Press, 1980", S. 175. 


\section{Markt- und Politikversagen}

Es ist vielfach zweifelhaft, ob Marktversagen durch politische Planung und Steuerung korrigiert werden kann. Es ist ebenso zweifelhaft, ob denn überhaupt in allen Fällen ein Marktversagen vorliegt. Handelt es sich bereits wirklich um Marktversagen, wenn die räumliche Allokation von dem Leitbild der staatlichen Planung abweicht? Ist die räumliche Allokation lediglich das Ergebnis von Marktkräften, oder spielen nicht auch politisch gesetzte Regeln und Anreize eine Rolle? Eine genaue Analyse der Prozesse zeigt, daß die Märkte ihre steuernde Funktion oftmals gar nicht wahrnehmen können. Durch die bewußte Milderung von Agglomerationsnachteilen und die Erhaltung bzw. Stabilisierung von Verdichtungsräumen werden die Preise der Produktionsfaktoren verfälscht. Paul Klemmer kommt daher auch zu folgendem Schluß: "Die suboptimale Regionalstruktur ist somit mehr ein Politik-, als ein Marktversagen." ${ }^{8}$ Dies führt im Ergebnis zu anderen wirtschaftspolitischen Konzeptionen. Nicht ein Mehr an politischer Planung und Steuerung ist gefragt, sondern eine Verringerung der politischen Einflußnahme und die stärkere ordnungspolitische Orientierung regionalpolitischer Ansätze.

\section{Ordnungspolitik}

Ordoliberale Ökonomen wenden sich entschieden gegen die These des Marktversagen und der konstruktivistischen Annahme der Planbarkeit und Steuerbarkeit wirtschaftlicher Prozesse. Der wirtschaftliche Entwicklungsprozeß wird als ein evolutorischer Prozeß verstanden, dessen Ergebnisse nicht prognostizierbar sind. Motor dieser Entwicklung ist der regelgebundene marktwirtschaftliche Koordinierungsprozeß zwischen den unzähligen Akteuren einer Volkswirtschaft. Er garantiert eine effiziente Allokation der Faktoren. Dies gilt auch für die räumliche Allokation. Die Politik erliegt aus dieser Sicht immer wieder der Versuchung, statt allgemeine Regeln zu setzen, wirtschaftliche Prozesse bewußt zu planen und zu steuern. Diese Versuche scheitern an den ,permanenten Grenzen unseres Tatsachenwissens ${ }^{\text {" } 9}$. Sie machen es den politischen Akteuren unmöglich, die relevanten Faktoren zu kennen, die die wirtschaftliche Entwicklung in einer Region beeinflussen. Wenn aber die relevanten Faktoren nicht bekannt sind, kann es auch kein Programm geben, das eine Region entsprechend eines wirtschaftlichen Leitbildes entwickelt. Somit gibt es keine Legitimation für regionalpolitische Programme.

\subsection{Regionalpolitik als öffentliches Gut}

Nicht nur die der Regionalpolitik zugrunde liegenden wirtschaftspolitischen Konzepte sind von Bedeutung, es gilt auch zu prüfen, ob Regionalpolitik ein öffentli-

$8 \quad$ Paul Klemmer: "Regionalpolitik auf dem Prüfstand", 1986, S. 30.

9 F.A. von Hayek: „Recht, Gesetzgebung und Freiheit: Band 1: Regeln und Ordnung“, S.27. 
ches Gut ist. Handelte es sich hier um ein solches, wäre eine Legitimation staatlichen Handelns gegeben.

Wie oben erwähnt, werde ich mich in dieser Arbeit auf die Regionalpolitik im Rahmen des EFRE, der GA sowie gleichartiger Landesprogramme konzentrieren. Die Programme beinhalten eine breite Palette unterschiedlicher Förderarten (wie wird gefördert) und Fördertatbeständen (was wird gefördert). Etwa 50\% der Mittel werden für die Förderung der wirtschaftsnahen Infrastruktur eingesetzt. Da dieser Begriff sehr weit interpretiert wird, können u.a. Gewerbegebiete, Kläranlagen, Straßen oder touristische Basisinfrastruktur bezuschußt werden. Die Förderung erfolgt über Zuschüsse oder über verbilligte Kredite. Ferner werden Unternehmensinvestitionen direkt gefördert. Auch hier werden Zuschüsse oder verbilligte Kredite eingesetzt.

Im Bereich der wirtschaftsnahen Infrastruktur ist nur die Förderung der öffentlichen Hand möglich. Die Programme unterstellen somit implizit, daß es sich hier um öffentliche Güter handelt, die von Privaten nicht bereitgestellt werden können. In der Literatur finden sich vier Kriterien für die Abgrenzung zwischen öffentlichen und privaten Gütern. Dies sind:
a. Ausschlußprinzip gilt nicht
b. Unteilbarkeit
c. Nichtrivalität im Konsum
d. Externe Effekte

Wie die Theorie der öffentlichen Güter zeigt, gibt es nur wenige rein öffentliche Güter. In den meisten Fällen handelt es sich um Güter, die Eigenschaften von privaten und von öffentlichen Gütern aufweisen. Dies gilt insbesondere für die im Rahmen der wirtschaftsnahen Infrastruktur geförderten Investitionen der öffentlichen Hand. Der Bau von Straßen fällt sehr deutlich in den Bereich der öffentlichen Güter, obwohl selbst in diesem Bereich teilweise eine privates Angebot möglich ist. Die Erschließung von als Gewerbegebiet ausgewiesenen Flächen ist hingegen eher den privaten Gütern zuzuordnen. Die Flächen werden an die Nutzer verkauft, so daß das Ausschlußprinzip jederzeit gewährleistet werden kann. Auch die Nichtrivalität im Konsum ist nicht gegeben. Die Grenzkosten eines zusätzlichen Nutzers sind eindeutig größer als Null. Es kann nur eine begrenzte Anzahl von Unternehmen auf der vorhandenen Fläche bauen. Je mehr Nutzer vorhanden sind, desto schwieriger wird es für den einzelnen Unternehmer, sein Projekt durchzuführen.

Das Vorliegen von externen Effekten ist ein umstrittenes Kriterium für die Abgrenzung von öffentlichen Gütern. Im Falle eines Gewerbegebietes ist es nicht das Gebiet an sich, sondern die private Nutzung, die die erhofften externen Effekte erzeugt. Obwohl die Förderrichtlinien ausdrücklich die Erschließung von Gewerbegebieten durch die öffentliche Hand vorsehen, handelt es sich um private Güter. 
Die Einordnung der Unternehmenssubventionen über Zuschüsse oder verbilligte Kredite ist etwas komplizierter. Der Ausschluß ist möglich, die Zuschüsse sind teilbar, und es herrscht eindeutig eine Rivalität im Konsum. Die Zuteilung von Zuschüssen wird ganz bewußt von der Erfüllung bestimmter Bedingungen abhängig gemacht und jede Inanspruchnahme von Fördergeldern reduziert die Chancen der übrigen Antragsteller. Gemessen an den Kriterien handelt es sich um ein privates Gut. Betrachtet man den Zweck dieser Maßnahmen, kommt man zu einer differenzierteren Einordnung. Durch die Zuschüsse soll die räumliche Allokation von Kapital verändert werden. Im Ergebnis senken die Zuschüsse die Kapitalkosten der Unternehmen und können somit die Nachfrage nach Kapital bzw. Investitionen steigern. Ausgehend von einer - gemessen an dem regionalpolitischen Leitbild - zu geringen Nachfrage nach Investitionen in einer Region wird versucht, über eine Meritorisierung dieses Gutes die Nachfrage zu erhöhen. Zunächst hat diese Förderung betriebswirtschaftlich einen eindeutigen Effekt. Die Kapitalkosten für den Unternehmer sinken. $\mathrm{Ob}$ und wenn ja, welche externen Effekte durch eine derartige Förderung erzielt werden können - Reduzierung der Arbeitslosigkeit, Induzierung von Wirtschaftswachstum, Erhalt bzw. Steigerung von Arbeitseinkommen, Erhöhung des regionalen Steueraufkommens - ist sehr fraglich und im einzelnen nur bedingt nachweisbar.

Diese wenigen Beispiele zeigen sehr anschaulich, daß die pauschale Einordnung der Regionalpolitik als öffentliches Gut unzutreffend ist. Auch aus dieser Analyse läßt sich daher keine pauschale Legitimation für staatliches Handeln im Bereich der Regionalpolitik ableiten. Es gilt vielmehr, von Fall zu Fall zu prüfen, ob eine öffentliche Bereitstellung sinnvoll und notwendig ist. Ist dies nicht der Fall, wäre einer privaten Lösung stets der Vorrang zu geben.

\subsection{Zusammenfassung}

Abschließend lassen sich folgende Ergebnisse dieses Kapitels zusammenfassen:

a. Gemäß den bestehenden Definitionen umfaßt Regionalpolitik fast alle Politikbereiche, die einen Bezug zur wirtschaftlichen Entwicklung in einzelnen Regionen haben. Eine Analyse aller relevanten Politikbereiche wäre im Rahmen dieser Arbeit nicht möglich. Die Arbeit konzentriert sich daher auf die Regionalpolitik, die im Rahmen des EFRE, der GA sowie vergleichbarer Landesprogramme durchgeführt wird.

b. Regionalpolitische Programme lassen sich anhand der ihnen zugrundeliegenden wirtschaftspolitischen Konzeption einordnen. Maßgebliches Kriterium hierfür sind die Annahmen, die über die Plan- und Steuerbarkeit wirtschaftlicher Prozesse gemacht werden. Auf dem einen Ende des Kontinuums steht das Konzept Marktversagen und Planbarkeit. Da die Marktkräfte zu einer suboptimalen regionalen Allokation führen, muß der Staat planend und steuernd eingreifen. Basierend auf einem regionalen 
Leitbild weist er den Teilräumen konkrete Funktionen $\mathrm{zu}$ und steuert somit die wirtschaftliche Entwicklung.

Das zweite Konzept geht primär von einem Politikversagen aus. Nicht Marktversagen, sondern der Einfluß von politisch gesetzten Anreizstrukturen führt $\mathrm{zu}$ den unerwünschten regionalen Disparitäten und einer $\mathrm{zu}$ starken Herausbildung von Agglomerationen. Es ist nicht ein Mehr an politischer Steuerung gefragt, sondern ein Abbau der Eingriffe und eine Abkehr von dem Versuch, wirtschaftliche Prozesse bewußt zu planen. Regionalpolitik muß stärker als Ordnungspolitik begriffen werden.

Am deutlichsten unterscheidet sich der ordnungspolitische Ansatz von der These der Planbarkeit und des Marktversagens. Eine Planbarkeit und Steuerbarkeit wirtschaftlicher Entwicklung ist nicht gegeben, sie verläuft vielmehr im Rahmen eines evolutionären Prozesses. Die regelgebundene Koordinierung der unzähligen einzelwirtschaftlichen Entscheidungen führt zu einer effizienten Allokation. Die wichtige Funktion der politischen Akteure ist die Setzung und Durchsetzung dieser Regeln.

c. Regionalpolitik läßt sich nicht pauschal als öffentliches Gut charakterisieren. Eine Analyse von Beispielen zeigt, daß es neben öffentlichen Gütern auch private und meritorische Güter gibt. Es bedarf einer Einzelfallprüfung, um eine Zuordnung vorzunehmen und gegebenenfalls staatliches Handeln zu legitimieren.

\section{Theoretische Fundierung der Regionalpolitik und unternehmerischer Standortentscheidungen}

Die Entwicklung der Wirtschaft im Raum sowie die Allokation der mobilen Produktionsfaktoren verläuft nicht gleichmäßig. Es bilden sich Zentren mit hohem wirtschaftlichem Wachstum und hoher Attraktivität für mobile Produktionsfaktoren heraus. Andere Bereiche sind weniger attraktiv, können nur wenige mobile Produktionsfaktoren anziehen oder verlieren diese im schlechtesten Fall sogar: es kommt zur Abwanderung von Kapital und Arbeitskräften. Das Kapital ist eindeutig den mobilen Produktionsfaktoren zuzuordnen. Bei den Arbeitskräften muß eine differenzierte Betrachtung vorgenommen werden. Grundsätzlich ist der Mobilitätsgrad der Arbeitskräfte in Deutschland nicht sehr hoch. Er steigt tendenziell mit dem Grad der Qualifikation an. Familiäre Bindungen und die Angst, das gewohnte soziale Umfeld zu verlieren, sind selbst für Arbeitslose ein wichtiger Grund, in ihrer Heimatregion zu bleiben.

Dieser Befund gilt grundsätzlich auch für die neuen Bundesländer. Allerdings gibt es eine Vielzahl von Menschen, die Ausbildungsplätze und Arbeit in den alten Bundesländern suchen. So wurde der ostdeutsche Arbeitsmarkt 1990/91 durch Umzüge nach Westdeutschland (ca. 300.000 Personen) und durch Tages- und 
Wochenpendler (500.000 Personen) stark entlastet. ${ }^{10}$ Ein Großteil des Arbeitskräftepotentials ist jedoch eher als immobiler Produktionsfaktor zu interpretieren. Die Mobilität wird im wesentlichen durch drei Faktoren begrenzt. Neben den oben bereits erwähnten sozialen Bindungen beschränkt die Aufnahmefähigkeit des westdeutschen Arbeitsmarktes den Umfang der Wanderung bzw. der Pendler. Ferner bestimmt die vorhandene Qualifikation des Arbeitnehmers die Chancen und den Willen zur Arbeitsplatzsuche außerhalb des gewohnten Umfeldes.

In den neuen Bundesländern stand und steht die Wirtschaftspolitik vor einer enormen Herausforderung. Die durch die sozialistische Planwirtschaft geschaffenen Wirtschaftsstrukturen brachen zusammen und deren Reste konnten dem Druck des Wettbewerbs nicht standhalten. Der rapide Rückgang der Industriearbeitsplätze verdeutlicht diese Entwicklung besonders anschaulich. Von 131 Beschäftigten in der Industrie je 1000 Einwohner 1991 waren 1992 nur noch 54 übrig. ${ }^{11}$ Der Entwertung des Kapitalstocks standen keine gleichwertigen Zuflüsse an neuem Kapital gegenüber. Da die Mobilität der Arbeitskräfte nur sehr eingeschränkt vorhanden ist und eine Entleerung der ostdeutschen Regionen bzw. ein "brain drain" deren Chancen für eine erfolgreiche wirtschaftliche Entwicklung weiter reduzieren würde, steht die Wirtschaftspolitik vor der Aufgabe, Anreize für den Aufbau eines neuen, leistungsfähigen Kapitalstockes zu geben.

Die Auswahl adäquater Mittel zur Erreichung dieses Zieles setzt voraus, daß man die ökonomischen Wirkungszusammenhänge kennt, die zu der räumlich stark differenzierten Entwicklung führen. Es bedarf also einer Theorie, die als Größe den Raum in der Analyse berücksichtigt.

Zur theoretischen Fundierung der Regionalpolitik gibt es eine Vielzahl unterschiedlicher Ansätze. ${ }^{12}$ Diese lassen sich in statische und dynamische Theorien unterteilen. $\mathrm{Zu}$ den statischen Theorien gehören u.a. die verschiedenen Standorttheorien. Deren Ursprung liegt in der 1826 durch Thünen entwickelten landwirtschaftlichen Standorttheorie, die später Grundlage für eine Standorttheorie der

Vgl. hierzu Deutscher Sparkassen- und Giroverband: "Ostdeutsche Wirtschaft im Wandel: Bestandsaufnahme und Perspektiven eines Aufholungsprozesses", Stuttgart 1992, S. $130 / 131$.

In Westdeutschland verringerte sich dieser Wert im gleichen Zeitraum von 116 auf 112 . Vgl. "Kahlschlag verhindern - industrielle Kerne erhalten", WSI Materialien Nr. 36, S. 19.

Einen sehr guten Überblick über die unterschiedlichen Ansătze und deren wirtschaftspolitischen Implikationen gibt Christiane Krieger-Boden: "Neue Argumente fur Regionalpolitik? Zur Fundierung der Regionalpolitik in ălteren und neueren regionalokkonomischen Theorien", in: Die Weltwirtschaft, Heft 2/1995, S. 193 ff. 
Städte geworden ist. Eine zweite Säule ist die von W. Launhardts und A. Weber entwickelte Industriestandortlehre. Die Theorie der räumlichen Konkurrenz, die die Frage der optimalen Verteilung der Anbieter eines Gutes im Raum behandelt, bildet die dritte Säule. Die vierte und letzte Säule wurde von W. Christaller begründet. Seine Theorie der zentralen Orte weist die Angebotsorte aus, an denen insbesondere staatliche Dienstleistungen angeboten werden sollten. ${ }^{13}$

Die dynamischen Theorien spielen für die Legitimierung regionalpolitischen Handelns eine größere Rolle als die statischen. ${ }^{14} \mathrm{Zu}$ ihnen zählen die Theorie der Wirtschaftsstufen, die neoklassische Wachstumstheorie, die Polarisationstheorien sowie die Theorie der öffentlichen Güter. Insbesondere die Polarisationstheorien spielen in der regionalpolitischen Diskussion eine bedeutende Rolle, da "... aus ihnen eine direkte Aufforderung zum regionalpolitischen Handeln herauszulesen ist ...". ${ }^{15}$

Die Frage nach dem richtigen Standort einer Unternehmung ist auch Gegenstand betriebswirtschaftlicher Untersuchungen. Die betriebswirtschaftlichen Standorttheorien gehen auf die von A. Weber und W. Launhardts entwickelte Industriestandortlehre zurück. Betriebswirtschaftliche und volkswirtschaftliche Theorien mit Raumbezug haben somit teilweise die gleiche Basis. Die betriebswirtschaftliche Standorttheorie läßt sich in deskriptive und präskriptive Ansätze unterteilen.

Es ist nicht Ziel dieser Arbeit, alle oben angeführten Theorien zu analysieren. Im Rahmen dieser Untersuchung sind nur die Theorien relevant, die zur Begründung von regionalpolitischen Ansätzen und Programmen herangezogen werden. Dies ist zum einen die Theorie der zentralen Orte, die Exportbasistheorie sowie die Theorie der Wachstumspole. Ferner sind die neuen Wachstumsmodelle von Bedeutung, da sie neue theoretische Überlegungen zur Fundierung der Regionalpolitik bieten. Alle Theorien werden zunächst kurz skizziert. Die Analyse konzentriert sich dann auf zwei Fragen:

a. Liefern die Ansätze eine schlüssige Begründungen für regionalpolitische Programme?

b. Welches wirtschaftspolitische Konzept liegt der Theorie zu Grunde?

Abschließend wird noch geprüft, ob und inwieweit betriebswirtschaftliche Theorien eine Orientierungshilfe für regionalpolitische Programme sein können. Wenn Regionalpolitik einzelwirtschaftliche Entscheidungen beeinflussen will, kann das

13 Vgl. Edwin v. Böventer: "Raumwirtschaft I: Theorie", a.a.O., S. 409.

14 Vgl. Christiane Krieger-Boden: "Neue Argumente furr Regionalpolitik? Zur Fundierung der Regionalpolitik in ălteren und neueren regionalökonomischen Theorien", a.a.O., S. 197. 
Wissen um die maßgebliche Faktoren, die diese Entscheidungen determinieren, sehr hilfreich sein. Auch hier wird geprüft, ob diese Theorien ein tragfähiges Fundament für regionalpolitische Programme sein können.

\subsection{Die Theoretische Fundierung der Regionalpolitik}

\subsubsection{Theorie der zentralen Orte}

Die Theorie der zentralen Orte beruht auf einer Untersuchung Christallers in Süddeutschland (1933). Ein zentraler Ort ist Mittelpunkt eines Gebietes. Die Zentralität ergibt sich aus der Überschußfunktion im Angebot zentraler Güter und Dienste für die nähere Umgebung. Der Schwerpunkt der Theorie liegt auf dem tertiären Sektor, da Dienstleistungen weder transportiert noch gelagert werden können. Die Theorie der zentralen Orte ist daher eine Standorttheorie des tertiären Sektors, wobei unerheblich ist, ob es sich um öffentliche oder private Güter handelt.

Die Theorie folgt einem absatzorientierten Ansatz, d.h. die zentralen Güter sollen bei möglichst geringen Transportkosten den Nutzern zur Verfügung gestellt werden können. Christaller unterscheidet in die obere und untere Reichweite eines zentralen Gutes. Die obere Reichweite gibt die Entfernung an, die die Konsumenten bereit sind, für ein zentrales Gut zurückzulegen. Die untere Reichweite wird durch die Mindestmenge bestimmt, die von einem zentralen Gut abgesetzt werden muß, damit die Produktion rentabel erfolgen kann. ${ }^{16}$ Basierend auf der Annahme, $\mathrm{da} ß$ die Bevölkerung gleichmäßig im Raum verteilt ist und die Transportkosten proportional zur Entfernung sind, kommt Christaller zu den bekannten sechseckigen Flächen, in deren Mitte die zentralen Orte liegen.

Die Theorie der zentralen Orte liefert keine Begründung für regionalpolitisches Handeln. Dies gilt sowohl allokationspolitisch, da durch die Entstehung zentraler Orte eine optimale Versorgung der Bevölkerung gewährleistet ist, als auch distributionspolitisch, da die Verteilung von Einkommen im Rahmen dieser Theorie keine Rolle spielt. Die Theorie der zentralen Orte kann bei der betriebswirtschaftlichen Kalkulation öffentlicher Leistungen nützlich sein, geht es doch letztendlich darum, bei einem gegebenen Absatzraum mit gegebener Bevölkerungsverteilung und gegebener Kostenstruktur eine optimale Standortwahl für die Bereitstellung einer öffentlichen Leistung zu treffen.

Regionalpolitisch bedeutsam ist die Erkenntnis, daß es wirtschaftlich effizient ist, das Angebot von Dienstleistungen in zentralen Orten zu konzentrieren. Die Theorie der zentralen Orte kommt somit bei der Frage der Implementation von Förderprogrammen zum Tragen. Sie bietet eine Methode, mit der die Förderung des tertiären Sektors hinsichtlich der räumlichen Verteilung effizient gestaltet werden kann. 
Ein wirtschaftspolitisches Konzept ist bei dieser Theorie nicht feststellbar, da es sich im wesentlichen um einzelwirtschaftliche Entscheidungen handelt, die für die Standortwahl von Krankenhäusern, Amtsgerichten oder Schwimmbädern relevant sind.

\subsubsection{Die Exportbasistheorie}

Die Exportbasistheorie zählt zu den Wirtschaftsstufentheorien. Nach der Exportbasistheorie sind Exportströme der entscheidende Faktor für Wohlstand, Wachstum und Entwicklung in einer Region. Diese exportorientierten Aktivitäten werden als Basisaktivitäten bezeichnet, die übrigen als sekundäre Aktivitäten (NonBasic-Activities). Die Exportbasistheorie geht auf die Lehre von den Entwicklungsstufen zurück. Die Wirtschaft einer Region entwickelt sich hiernach von der autarken Familienwirtschaft über die Bauern- und Handwerkerwirtschaft hin zu einer arbeitsteilig organisierten modernen Wirtschaft. Im Zuge dieser Entwicklung sind die Städte nicht mehr in der Lage, sich selbst zu versorgen. Um den Import von Lebensmitteln und Vorprodukten bezahlen zu können, müssen Güter exportiert werden. ${ }^{17}$

Für Amerika hält D. North dieses Konzept für nicht zutreffend. Ausschlaggebend waren hier die Vorkommen an natürlichen Reichtümern, die exportiert wurden. Über diesen Export wurde die Entwicklung anderer Sektoren der Wirtschaft finanziert. ${ }^{18}$ Die Bestimmung der Basisaktivitäten ist nicht exakt möglich. Die Unternehmen einer Region, die für den Export produzieren, gehören gemäß der Theorie zu den Basisaktivitäten. Es ist unbedeutend, ob sie aus dem tertiären, sekundären oder primären Bereich stammen. Aus der formalen Darstellung ergibt sich, daß das Einkommen einer Region ausschließlich von den Exporterlösen abhängt. Hierbei wird eine konstante marginale Konsumquote für innerregionale Konsumgüter unterstellt. ${ }^{19}$

Auch diese Theorie hat ihre Schwächen. Das größte Problem liegt in der Abgrenzung der Region, die als "Inland" gelten soll. Je kleiner eine Region ist, desto mehr Exportmöglichkeiten bestehen. Kleinere Einheiten müßten somit größere Wachstumspotentiale haben.

Ferner bleibt unberücksichtigt, daß auch durch eine Importsubstitution positive Einkommenseffekte erzielt werden können. Bei gegebenen Exporten und einer vom Gesamteinkommen abhängigen Produktion im Non-Basic-Sektor, führt eine

Vgl. Bernhard Oswald: "Erfolgskontrolle in der Regionalpolitik", a.a.O., S. 72.

Vgl. D. North: "Location Theory and Regional Economic Growth", in JPE, Bd 23, 1955, S. 243-258.

Das Einkommen einer Region $\mathrm{Y}(\mathrm{L})$ ist dann wie folgt definiert: $\mathrm{Y}(\mathrm{L})=\mathrm{Y}(\mathrm{EX})(1 / 1-b-1)$. $(b=$ marginale Konsumquote fur heimische Guter, $Y(E X)=$ Exporteinkommen). 
Importsubstitution zu einer Einkommenssteigerung in der Region. ${ }^{20}$ Ferner zeigt dieses Beispiel, daß die Relation zwischen Basic und Non-Basic-Activities nicht konstant sein muß, sondern sich durchaus verändern kann.

Die Kapitalströme, die mit dem Export und Import verbunden sind, bleiben in der Analyse unberücksichtigt. Nicht immer führt ein Exportüberschuß auch zu einer positiven Zahlungsbilanz. Steigende Exporte können mit steigenden Importen Import von Vorprodukten - verbunden sein. Ist die Zunahme der Importe größer als die Zunahme der Exporte, ergibt sich keine Verbesserung der Zahlungsbilanz. ${ }^{21}$ Neben den exportorientierten Betrieben sind auch die innerregional orientierten Betriebe von besonderer Bedeutung, da sie der Ursprung für die Basisaktivitäten sind. Steigt die Exportnachfrage an, so muß die Region in der Lage sein, hierauf zu reagieren. Dies kann sie nur, wenn nicht voll ausgelastete Produktionskapazitäten vorhanden sind bzw. die Produktionsfaktoren nicht voll beschäftigt sind. Siebert weißt daraufhin, daß Exportgüter zunächst regionale Güter gewesen sein müssen, um dann im Zuge eines Entwicklungsprozesses zu Exportgütern zu werden. $^{22}$

Auch aus dieser Theorie lassen sich weder allokationspolitische noch distributionspolitische Begründungen für eine Regionalpolitik ableiten. Sie liefert keine Erklärung für die bestehenden wirtschaftlichen Disparitäten, sondern ein Modell für den Verlauf eines Entwicklungsprozesses. Das wirtschaftspolitische Konzept, das einer Regionalpolitik, die sich auf die Exportbasistheorie beruft, zugrundeliegt, ist ganz eindeutig ein Konzept der bewußten Planung und Steuerung wirtschaftlicher Abläufe. Die Theorie unterstellt ein bestimmtes Entwicklungsmuster für Volkswirtschaften. Ferner wird ein Versagen der Marktkräfte angenommen, da die Exportchancen nicht hinreichend genutzt bzw. nicht erkannt werden. Der politische Planer muß diese Defizite ausgleichen. Er muß die Branche bzw. das Unternehmen mit den besten Exportchancen auswählen und diese dann gezielt fördern, damit ein wirtschaftlicher Entwicklungsprozeß angestoßen werden kann. Dieser konstruktivistische Politikansatz versucht, in Unkenntnis der Ursachen für die wirtschaftlichen Disparitäten, einen Entwicklungsprozeß gemäß dem Modell zu initiieren. Es liegt auf der Hand, daß dem politischen Planer die notwendigen Informationen für eine Identifikation und Förderung der Exportbasis fehlen.

Vgl. N. Irsch, B. Muller-Kåstner: "Vorschläge zur Weiterentwicklung der regionalen Wirtschaftspolitik", in Wirtschaftsdienst, 1984, H. 12, S. $626 \mathrm{f}$ sowie Bernhard Oswald, "Erfolgskontrolle in der Regionalpolitik", a.a.O., S 80-82.

Vgl. H.W. Richardson: "Regional Growth Theory", London 1973, S. 17f.

Vgl. Horst Siebert: "Zur Theorie regionalen Wirtschaftswachstums", Diss., Münster 1965, S. 91 . 


\subsubsection{Die Konzeption der Wachstumspole}

Einen wesentlichen Einfluß auf die theoretische Fundierung der Regionalpolitik haben die Polarisierungstheorien. Sie gehen davon aus, daß einmal eingetretene Ungleichgewichte dazu neigen, "... sich zirkulär zu verstärken und zu einer starken Polarität zwischen Zentren einerseits und der Peripherie andererseits zu führen."23

Die Theorie geht von der Erkenntnis aus, daß wirtschaftliches Wachstum nicht überall zum gleichen Zeitpunkt auftritt, sondern es Punkte bzw. Pole mit unterschiedlicher Wachstumsintensität gibt. ${ }^{24}$ Die Wirkungen von Wachstumspolen breiten sich durch verschiedene Kanäle aus.

Im Zentrum der Theorie der Wachstumspole steht der Begriff der "motorischen" Firmen. Dies sind Firmen, die Einfluß auf andere Firmen ausüben und starke marktmäßige und außermarktliche Beziehungen haben. Die Konzentration von motorischen Industrien oder eines Industriekomplexes an einem Ort wird als Wachstumspol bezeichnet. "Es handelt sich dabei also um einen räumlich lokalisierten, (kräftig) expandierenden, funktionalen Komplex von interindustriellen, kommerziellen und finanziellen Beziehungen, der über die Märkte und Informationsmedien andere Wirtschaftseinheiten im umgebenden Raum direkt und/oder indirekt beeinflußt." 25

Die motorischen Industrien werden primär dem Sekundärbereich zugeordnet. Sie geben mehr Impulse ab als sie empfangen. Der Pol beeinflußt die sektorale und regionale Struktur über technische, ökonomische und psychologische Kanäle. Die Theorie der Wachstumspole ist eine Multiplikatortheorie des Sekundärbereichs ${ }^{26}$ sowie eine Theorie der externen Effekte von Wachstumspolen.

Die Theorie vernachlässigt, daß zur Ansiedlung von motorischen Industrien in einer Region das Bestehen von positiven externen Effekten nicht ausreicht. Es müssen auch andere Standortfaktoren "... wie die Infrastrukturausstattung, das Angebot von sowie die Nachfrage nach unterschiedlich qualifizierten Arbeitskräften oder die Entfernung zu den Märkten gebührend berücksichtigt werden." ${ }^{27}$

Die Theorie der Wachstumspole bietet sowohl eine distributionspolitische als auch eine allokationspolitische Fundierung der Regionalpolitik. Ursache für die Entstehung von Wachstumspolen ist ein Versagen des Marktes, der nicht in der

Christiane Krieger-Boden: "Neue Argumente fur Regionalpolitik? Zur Fundierung der Regionalpolitik in ălteren und neueren regionalઠkkonomischen Theorien", a.a.O., S. 199.

Entstanden ist die Theorie der Wachstumspole in Frankreich. Sie wurde von F. Perroux 1955 entwickelt und von verschiedenen Autoren verfeinert.

Bernhard Oswald: "Erfolgskontrolle in der Regionalpolitik", a.a.O., S. 59.

Investitionen im Sekundärbereich vergrößern das Einkommen $(\mathrm{dY}=1 / \mathrm{s} \mathrm{dI}), \mathrm{Vgl}$. hierzu Bernhard Oswald, "Erfolgskontrolle in der Regionalpolitik", a.a.O., S. 60.

Bernhard Oswald: "Erfolgskontrolle in der Regionalpolitik", a.a.O., S. 70. 
Lage ist, die vorhandenen Ungleichgewichte abzubauen. Allokationspolitisch ist bedeutsam, daß dieses Marktversagen zu einer sich zirkulär verstärkenden Entwicklung führt, die im Ergebnis die Unterschiede zwischen Zentrum und Peripherie vergrößert. ${ }^{28}$ Die so entstandenen Disparitäten zwischen Zentrum und Peripherie sind dann der Grund für distributionspolitische Aktivitäten. Das Entstehen von Ungleichgewichten und sich zirkulär verstärkenden Entwicklungen kann jedoch auch als Politikversagen interpretiert werden. Politisch bestimmte Anreizstrukturen können derartige Entwicklungen verstärken und eine Entfaltung der Marktkräfte verhindern. Diese Interpretation ist allerdings nicht geeignet, regionalpolitische Programme zu legitimieren. Sie würde vielmehr zu einer Abschaffung vorhandener öffentlicher Regelungen und Leistungen führen.

Auch hier gilt: Einer Regionalpolitik, die sich auf die Theorie der Wachstumspole beruft, liegt ein wirtschaftspolitisches Konzept zugrunde, das an die bewußte Plan- und Steuerbarkeit wirtschaftlicher Entwicklungen glaubt. In der Anwendung der Theorie der Wachstumspole stellt sich das Problem der Identifikation motorischer Industrien. Die Theorie gibt keine Erklärung dafür, warum und wie motorische Industrien entstehen. Die regionalpolitischen Akteure stehen somit vor dem Problem, die richtige Industrie für eine Förderung auszuwählen. Aus ordnungspolitischen Erwägungen stellt sich, wie bei jeder staatlichen Förderung und jedem wirtschaftspolitischen Programm, die Frage der Informationsgewinnung. Können Behörden mit ihren Mitteln motorische Industrien besser identifizieren als der Markt? Nach welchem Kriterium wird eine motorische Industrie ausgewählt? Da die Theorie auf diese Fragen keine Antwort gibt, ist die Auswahl der zu fördernden Industrien eine rein politische Entscheidung.

Verschiedene Studien aus Frankreich belegen diese Problematik eindrucksvoll. Oswald führt z.B. die Ansiedlung von Erdgasindustrie im Raum Lacq (nördlich von Marseille) an. Die erhofften Ausstrahlungseffekte blieben aus, die Erdgasindustrie entwickelte sich nicht zu einem Wachstumspol. ${ }^{29}$ Eine ähnliche Frage stellt sich in Mecklenburg-Vorpommern für die Werftindustrie. Sind die Werften den motorischen Industrien zuzurechnen, können sie einen expandierenden Kern darstellen, der positive Ausstrahlungseffekte für das Wachstum der Region hat? Die Theorie der Wachstumspole führt die politischen Entscheidungsträger in die Dilemmata staatlicher Industriepolitik. Die Auswahl wachstumsstarker und innovativer Industrien wird nicht der in einer Marktwirtschaft hierfür zuständigen Institution, dem Markt, überlassen. Vielmehr müssen die politischen Institutionen, wollen sie dieses Konzept anwenden, dem Markt diese Entscheidung abnehmen. Regionalpolitik in älteren und neueren regionalokonomischen Theorien", a.a.O., S. 199. 
Somit basieren die Entscheidungen der politischen Planer nicht auf ökonomischen, sondern auf politik-ökonomischen Kalkülen.

\subsubsection{Neue Wachstumsmodelle}

Die Neuen Wachstumsmodelle haben auch der Regionaltheorie neue Impulse gegeben. In der "Neuen Wachstumstheorie" wird neben Arbeit und Kapital der technische Fortschritt als dritte endogene Variable zur Erklärung von Wachstumsprozessen herangezogen. ${ }^{30}$ Voraussetzung für ein beständiges Wachstum ist, daß der Anreiz zur Forschung und zum Einsatz neuer Technologien nicht mit zunehmenden Bestand an Technologien zurückgeht. ${ }^{31}$ Unter dieser Bedingung zeigt die gesamtwirtschaftliche Produktionsfunktion zunehmende Skalenerträge.

Mit der Berücksichtigung von Forschung und Entwicklung stellt sich auch die Frage der externen Effekte und ihrer Wirkungen auf regionalwirtschaftliche Agglomerationsprozesse. Die bisher diskutierten Modelle, die die Verteilung von Industrien im Raum, also die Erklärung von Agglomerationen, zum Gegenstand haben, nutzen die Transportkosten als die entscheidende Erklärungsvariable. ${ }^{32}$ Dies führt unter der Bedingung einer in einem Punkt konzentrierten Nachfrage zu einer kreisförmigen Anordnung der Industrien um ein Zentrum (Thünen - Ringe). Bei einer im Raum gleichmäßig verteilten Nachfrage ergeben sich wabenförmige Marktgebiete. Diese Bedeutung der Transportkosten läßt sich indes empirisch nicht belegen. Die Standortentscheidungen der Unternehmen hängen von vielen verschiedenen Faktoren ab. Welcher Faktor ausschlaggebend ist, wird durch die Branche, das Produkt, die Größe der Firma und durch institutionelle Rahmenbedingungen beeinflußt.

Neuere Ansätze, die das Entstehen von Agglomerationen erklären, führen diese auf historische Zufälligkeit und Pfadabhängigkeit zurück. ${ }^{33}$ So waren es in vielen Fällen "... unternehmerische und innovatorische Einzelleistungen, die das Entstehen von Branchenzentren auslösten. Ihnen allen ist gemein, daß einmal eingeschlagene Entwicklungsrichtungen eine erstaunliche Beharrungstendenz auf-

Vgl. Bert Hofman, Michael J. Koop: "Die 'Neue Wachstumstheorie' und ihre Bedeutung furr die Wirtschaftspolitik", in "Die Weltwirtschaft", Heft 2, 1991, S. 86-94.

Die Grenzertrăge des akkumulierbaren Faktors müssen daher konstant sein, vergl. Bert Hofman, Michael J. Koop: "Die 'Neue Wachstumstheorie' und ihre Bedeutung fur die Wirtschaftspolitik", a.a.O., S. 89.

Vgl. H. Klodt und K.-H. Paqué: "Am Tiefpunkt der Transformationskrise: Industrie- und lohnpolitische Weichenstellungen in den jungen Bundeslăndern", Kieler Diskussionsbeitrăge, 1993, S.8.

Vgl. hierzu Paul Krugmann, "History and industry location: The case of the US manufactoring belt", in: The American Economic Review, Vol. 81, 1991, S. 80-83. 
weisen....$^{134}$ Im Gegensatz zu den traditionellen Erklärungsansätzen für die Bildung von Agglomerationen, die einen gleichgewichtigen Anpassungsprozess zur Optimierung von Transportkosten unterstellen, werden hier Agglomerationen "... als Ergebnis ungleichgewichtigen Wachstums, das von stochastischen Ergebnissen ausgelöst wird", ${ }^{35}$ gesehen.

Durch die neuen Wachstumsmodelle rückte die Bedeutung von externen Effekten für die Entstehung von Agglomerationen in den Mittelpunkt des Interesses. Angelehnt an Erkenntnisse aus der Innovationsforschung und der Wachstumstheorie spielen in den neusten Modellen kommunikative Netzwerke eine besondere Rolle. Dieses Konzept entwickelt die Idee der Agglomerationsvorteile (Positive externe Effekte durch ein großes Angebot qualifizierter Arbeitskräfte, informelle Kommunikationsnetze etc.) weiter. Ein kommunikatives Netzwerk ist ein Geflecht von formellen und informellen Informationskanälen. Die positiven externen Effekte beschreiben Klodt und Paqué wie folgt: "Aus Sicht eines einzelnen Unternehmens stellt ein kommunikatives Netzwerk ebenso wie ein Pool qualifizierter Arbeitskräfte eine positive Externalität dar: Jedes Unternehmen profitiert hier von den Aktivitäten der anderen, ohne dafür einen Preis entrichten zu müssen ${ }^{136}$ Wenn Arbeitskräftepools und kommunikative Netzwerke für die wirtschaftliche Entwicklung einer Region von Bedeutung sind, dann kann es wirtschaftspolitisch sinnvoll sein, das Entstehen dieser Bedingungen zu fördern. Klodt und Paqué kommen zu dem Schluß: "Auf diese Weise läßt sich in vollem Einklang mit den ordnungspolitischen Regeln einer Marktwirtschaft eine aktive Regionalpolitik zugunsten von Regionen begründen, in denen es ohne Staatsförderung nicht zu Ansiedlungen von Unternehmen käme, die positive Externalitäten ausstrahlen. ${ }^{137}$

Der Reiz der neuen Wachstumsmodelle liegt darin, daß sie eine direkte Legitimation für regionalpolitisches Handeln liefern. Mit Hilfe regionalpolitischer Ansätze und Programme ist der politische Planer aufgefordert, Bedingungen zu schaffen, die zur Ansiedlung von Unternehmen mit positiven Externalitäten führen. Auch diese Theorie ist ein konstruktivistischer Ansatz, der die Plan- und Steuerbarkeit von externen Effekten voraussetzt. Auch hier steht die Wirtschafts- und Regionalpolitik vor der Aufgabe, die Industrien mit positiven Externalitäten zu identifizieren, damit eine erfolgreiche Förderung möglich wird. Fördert sie die Ansiedlung von Industrien, die keine oder im schlimmsten Fall sogar negative externe Effekte haben, führt dies nicht zu Wachstum und Entwicklung, sondern zu Stagnation und Rezession. Auch die neuen Wachstumsmodelle lösen das Informationsproblem

H. Klodt u. K.-H. Paqué: "Am Tiefpunkt der Transformationskrise", a.a.O., S. 8.

H. Klodt u. K.-H. Paqué: "Am Tiefpunkt der Transformationskrise", a.a.O., S. 8.

H. Klodt u. K.-H. Paqué: "Am Tiefpunkt der Transformationskrise", a.a.O., S. 10.

H. Klodt u. K.-H. Paqué: "Am Tiefpunkt der Transformationskrise", a.a.O., S. 10. 
nicht, sondern verschärfen es vielmehr. Basis für wirtschaftliche Entwicklung sind kommunikative Netzwerke, Arbeitskräftepools und sonstige positive Externalitäten. Es gibt keine Eingrenzung oder nähere Definition der Faktoren, die positive Externalitäten erzielen, es gibt - ganz im Gegenteil - eine breite Palette von potentiellen Möglichkeiten. Dies stellt den politischen Planer vor ein sehr großes Informationsproblem. Zunächst muß er die relevanten Externalitäten identifizieren und dann muß er die passende Maßnahme planen und implementieren. Das diese Aufgabe nicht lösbar ist und der konstruktivistische Ansatz zum scheitern verurteilt ist, wird offensichtlich. Doch, was aus ordnungspolitischer Sicht unheilvoll ist, ist aus politik-ökonomischer Sicht sehr wertvoll. Mit den neuen Wachstumsmodellen lassen sich fast alle regionalpolitischen Aktivitäten ökonomisch legitimieren, obwohl sie eigentlich politik-ökonomisch begründet sind.

\subsection{Die unternehmerische Standortentscheidung}

Die betriebswirtschaftlichen Standorttheorien betrachten die unternehmerische Standortentscheidung aus zwei Perspektiven. Gegenstand der deskriptiven Ansätze sind die Faktoren, die Standortentscheidungen maßgeblich beeinflussen. Die präskriptiven Ansätze entwickeln Modelle für eine optimale Standortplanung.

Für die Regional- und Strukturpolitik könnten die deskriptiven Ansätze von Interesse sein. Ziel dieser teilweise empirisch orientierten Arbeiten ist es, die für eine Entscheidung relevanten Standortfaktoren herauszuarbeiten. Bereits Weber versuchte 1909 einen Katalog der relevanten Standortfaktoren aufzustellen. In der Folge gab es eine Vielzahl von Vorschlägen für eine sinnvolle Systematisierung der Standortfaktoren. Einer der neueren Kataloge von Domschke und Drexel beinhaltet folgende Faktoren: ${ }^{38}$ Grund und Boden, Arbeitskräfte, Verkehr und Transport, Beschaffung und Entsorgung, Produktion, Absatz, Investition und Finanzierung, öffentliche Hand, allgemeine Infrastruktur und persönliche Präferenzen.

Der Katalog sagt noch nichts über die Gewichtung der einzelnen Faktoren aus, die nach Branchen und Betriebsgrößen sehr unterschiedlich sein kann. Es gibt verschiedene empirische Untersuchungen über die Bedeutung der einzelnen Standortfaktoren. Im Zentrum der jüngsten Untersuchungen steht zumeist die Bedeutung der sogenannten weichen Standortfaktoren. Dies sind Faktoren, die nicht in monetären oder anderen quantitativen Größen meßbar sind, sondern lediglich einer qualitativen Bewertung zugänglich sind. Im Rahmen einer Studie hat das Deutsche Institut für Urbanistik 1993 die Wichtigkeit von Standortfaktoren ermittelt. ${ }^{39}$ Unter den zehn Standortfaktoren, die Unternehmen für die wichtigsten halten, sind sechs den harten Faktoren zuzuordnen (Verkehrsanbindung, Arbeitsmarkt, Flä-

Vgl. W. Domschke, A. Drexl: "Logistik: Standorte", München 1990, sowie A. Drexl, "Standorttheorien", in Enzyklopădie der Betriebswirtschaftslehre, Stuttgart, 1993, S. $3965 f$.

O.V.: "Bedeutung weicher Standortfaktoren", Difu-Berichte 1/1994, S. 2-6. 
chen-/Bürokosten, Kommunale Abgaben, Flächen-/Büroverfügbarkeit, Kontakte zu Unternehmen) und vier den weichen Faktoren (Wohnen und Wohnumfeld, Umweltqualität, wirtschaftspolitisches Klima, Unternehmensfreundlichkeit der kommunalen Verwaltung).

Die Difu Studie zeigt jedoch auch sehr deutlich, daß die Bedeutung der Standortfaktoren nicht für alle Unternehmen gleich ist. Die Bewertung der Standortfaktoren ist z.B. abhängig von der Branche und der Betriebsgröße. Auch zwischen Westdeutschland und Ostdeutschland gibt es geringe Bewertungsunterschiede. Von den 26 abgefragten Standortfaktoren werden nur sieben in Ostdeutschland als deutlich wichtiger eingestuft. Bei den harten Faktoren gilt dies für die Nähe zu Absatzmärkten, den Arbeitsmarkt, die Verfügbarkeit qualifizierter Arbeitnehmer und die Subventionen und Fördermittel. Fast alle weichen unternehmensbezogenen Faktoren werden als wesentlich bedeutsamer beurteilt als im Westen. ${ }^{40}$

Die empirischen Untersuchungen zur Standortwahl können der Regional- und Strukturpolitik einen Anhalt geben, welche Standortfaktoren verbessert werden müßten, damit eine Ansiedlung von Unternehmen befördert werden könnte. Allerdings sind die Ergebnisse der Untersuchungen sehr instabil und weisen sowohl regionale wie auch sektorale Besonderheiten auf. Sie können somit nicht die Informationsprobleme lösen, die der politische Planer bei der Anwendung regionalpolitischer Theorien hat.

\subsection{Zusammenfassung}

Es zeigt sich recht deutlich, daß jede Theorie nur einen eingeschränkten Erklärungsgehalt für die Entstehung regional unterschiedlicher Wirtschaftskraft hat. Dies wird ganz besonders deutlich, wenn mit Hilfe komplizierter ökonometrischer Modelle eine Evaluation von regionalpolitischen Programmen vorgenommen wird. Basis eines ökonometrischen Modells ist die Kenntnis um den regionalen Funktionszusammenhang. Dieser kann nur so gut sein wie die Regionaltheorie. Da diese jedoch nicht in der Lage ist, Modelle zu entwickeln, die die regionale Wirtschaftsentwicklung erklären können, basieren die ökonometrischen Untersuchungen auf vielfältigen Annahmen, die ihre Aussagefähigkeit sehr stark einschränken. $^{41}$

Regionalwirtschaftliche Modelle können immer nur partialanalytische Modelle sein. Welchen Erklärungsgehalt die unabhängige Variable hat, hängt auch von der

O.V.: "Bedeutung weicher Standortfaktoren", Difu-Berichte 1/1994, S. 5.

41

Diese Verfahren scheitern theoretisch an einem fehlenden Regionalmodell, methodisch an fehlenden statistischen Material und empirisch an den Problemen der Datenbeschaffung. Siehe auch Klaus Hembach: "Der Stellenwert von Wirkungsanalysen fur die Regionalpolitik - Eine Systematisierung der Problematik am Beispiel der regionalen Wirtschaftspolitik", 1980, S. 53 f. 
Lage, der Struktur und den Institutionen einer Region ab sowie von der allgemeine volkswirtschaftlichen Situation. Basis der einen Modelle sind die Transportkosten, die die räumliche Verteilung determinieren. Der Einfluß der Transportkosten ist abhängig von der Art der Produktion, dem Stand der Verkehrstechnik sowie dem Ausmaß der Internalisierung externer Effekte. Die Verlagerung der Lagerhaltung auf die Straße im Rahmen der ,just-in-time-Fertigung" war unter anderem deshalb wirtschaftlich sinnvoll, weil sich im Preis für Transportleistungen nicht alle externen Kosten widerspiegeln.

Zentrales Argument der anderen Modelle sind externe Effekte, die durch Agglomerationsvorteile bzw. -nachteile entstehen. Bei den Polarisierungsmodellen ist Marktversagen die Ursache für eine nicht optimale Allokation der Produktionsfaktoren im Raum. Es ist allerdings sehr zweifelhaft, ob hier ein reines Marktversagen vorliegt.

Die neusten Ansätze, die auf der neuen Wachstumstheorie aufbauen, sehen positive externe Effekte als notwendige Bedingung für die Entstehung regionaler Wirtschaftszentren und somit für regionales Wachstum an. Sie liefern jedoch keine Anhaltspunkte für die Auswahl der Industrien bzw. der Dienstleistungen, die diese externen Effekte erzeugen können. Auch diese Modelle überlassen die Förderentscheidung letztendlich der politischen Rationalität.

Eine schlüssige theoretische Fundierung regionalpolitischer Aktivitäten liefern die herkömmlichen und auch die neueren Ansätze nicht. Die Theorien, die zur Begründung regionalpolitischer Aktivitäten herangezogen werden können, die Polarisierungstheorien sowie die neuen Wachstumsmodelle, weisen erhebliche Defizite bezüglich ihrer praktischen Umsetzbarkeit auf. Für beide Ansätze ist eine "... Vielzahl von Informationen über Produktionsfunktionen, Nachfrageverhalten, künftige Innovationen, Größe externer Effekte usw. ..." ${ }^{142}$ erforderlich, die jedoch nicht verfügbar sind.

Trotz der theoretischen Defizite und der massiven Informationsprobleme beruft sich die derzeit praktizierte Regionalpolitik implizit oder explizit auf eine oder mehrere der oben skizzierten Theorien. Regionalpolitik in Deutschland und Europa beruht auf konstruktivistischen wirtschaftspolitischen Konzepten. Es wird eine Planbarkeit und Steuerbarkeit regionaler wirtschaftlicher Entwicklung unterstellt. 


\section{Zuordnung regionalpolitischer Entscheidungs- und Finanzierungs- kompetenzen im föderalen Staat}

"Aber ich glaube, daß eine zentralisierte Verwaltung zu nichts anderem taugt, als die ihr unterworfenen Volker zu schwăçhen, denn sie vermindert in ihnen ohne Unterlaß den Bürgergeist." Alexis de Tocqueville

Obwohl die theoretische Basis ausgesprochen brüchig ist, und der Anspruch regionalpolitischer Programme an der komplexen Realität scheitert, wird auf allen Ebenen des politischen Systems versucht, die Entwicklung der regionalen Wirtschaft bewußt zu planen und zu steuern. Den regionalpolitischen Ansätzen aller Ebenen liegt fast ausnahmslos ein konstruktivistischer Politikansatz zugrunde. Es finden sich nur sehr wenige Elemente ordnungspolitischer Ansätze, die auf bewußte Steuerung und Planung verzichten. Die Fehlsteuerung dieser Ansätze kann durch eine falsche Zuweisung der relevanten Kompetenzen noch verschärft werden. Ausgehend von diesen Rahmenbedingungen gilt es zu prüfen, auf welcher Ebene regionalpolitische Kompetenzen angesiedelt werden müßten, um das Aus$\mathrm{ma} ß$ der Fehlsteuerung zu reduzieren. Hierzu wird grundsätzlich die Frage abgewogen, ob eine zentrale oder dezentrale Zuweisung von Entscheidungs- und Finanzierungskompetenzen sinnvoll ist. Die in der Literatur entwickelten Kriterien werden vorgestellt und auf ihre Anwendbarkeit geprüft.

Ferner gilt es, alternativ ein ordnungspolitisches Konzept der Regionalpolitik zu entwickeln. Basis hierfür ist das ordnungspolitisch fundierte Wettbewerbskonzept Hayeks. Dieses Modell wird auf die Beziehung zwischen regionalen staatlichen Organisationen angewandt. Das in diesem Abschnitt dargestellte Konzept des Wettbewerbs zwischen regionalen Einheiten wird im weiteren Verlauf der Arbeit Maßstab für die Analyse der Regionalpolitik in Mecklenburg-Vorpommern sein.

\subsection{Zentrale versus dezentrale Regionalpolitik}

Im Rahmen der Theorie des fiscal federalism sind Kriterien für die Zuordnung von Kompetenzen in einer föderalen Struktur entwickelt worden, die auch Maßstab für die Zuordnung regionalpolitischer Kompetenzen sein können. Ein Kriterium ist die Internalisierung externer Effekte eines öffentlichen Gutes, ein anderes die Berücksichtigung der regionalen Präferenzen. Beide Kriterien werden kurz dargestellt und ihre Wirkung analysiert.

Ein wichtiges Kriterium für die Zuteilung von Kompetenzen sind externe Effekte bzw. deren Internalisierung. Die effiziente Allokation eines öffentliches Gutes ist nicht sichergestellt, wenn "... die Grenzen einer Jurisdiktion mit der Reichweite

43 Alexis de Tocqueville: „Über die Demokratie in Amerika“ (1835), aus dem franzősischen von Hans Zbinden, Zürich 1987, Band 1, S. 127. 
eines von ihr angebotenen öffentlichen Gutes nicht deckungsgleich sind. ${ }^{44}$ In dieser Situation fallen Kosten und Nutzen eines öffentlichen Gutes auseinander, und es kann im Ergebnis zur Über- oder Unterversorgung kommen. ${ }^{45}$ Das Prinzip der fiskalischen Äquivalenz fordert, öffentliche Güter auf der Ebene anzubieten, die die Kosten der Bereitstellung trägt und den Nutzen aus ihnen zieht.

In arbeitsteiligen, durch vielfältige Interdependenzen geprägten Industriegesellschaften stößt dieses Prinzip jedoch sehr rasch an seine Grenzen. Ausmaß und Reichweite der externen Effekte sind für jedes öffentliche Gut verschieden. Dieses Prinzip konsequent zuende gedacht, würde bedeuten, daß für jedes öffentliche Gut "eine separate Regierungsinstitution" ${ }^{46}$ gefordert werden muß. Unter der Annahme, daß die Struktur des förderalen Systems langfristig als konstant anzusehen ist, erscheint eine optimale Internalisierung externer Effekte kaum möglich. Mit der Bildung von Zweckverbänden ist allerdings eine Annäherung an diese Forderung möglich.

Die ausschließliche Orientierung an der Internalisierung externer Effekte führt jedoch zu problematischen Ergebnissen. ${ }^{47}$ Es haben sich zwei Problemlösungsstrategien herausgebildet:

Die eine Strategie sucht, über die Zentralisierung der Kompetenzen die externen Effekte zu internalisieren. Hierbei muß man auf eine Ebene gehen, auf der keine Externalitäten mehr vorliegen. ${ }^{48}$ Tullock zeigt die Absurdität dieses Vorhabens anhand der Straßenreinigung, die "...möglicherweise nur im Rahmen eines Entscheidungskollektivs von kontinentalen oder planetarischen Ausmaßen erfolgen ..." ${ }^{49}$ könnte. Das Kriterium der bestmöglichen Internalisierung von Externalitäten

M. Olson: "Das Prinzip der fiskalischen Gleichheit: Die Aufteilung der Verantwortung zwischen verschiedenen Regierungsebenen", in G. Kirsch, "Föderalismus", 1977, S. 71.

Wenn der Nutzen uber die Jurisdiktion hinaus streut, die Kosten jedoch von ihr alleine zu tragen sind, kommt es zu einer Unterversorgung. Der umgekehrte Fall fuhrt zu einer Überversorgung.

M. Olson: "Das Prinzip der fiskalischen Gleichheit: Die Aufteilung der Verantwortung zwischen verschiedenen Regierungsebenen", a.a.O., S. 71.

Olson begreift daher sein Modell der fiskalischen Äquivalenz auch als Vormodell und warnt vor der direkten Übertragung in die Realität: "Es ist selten angebracht, direkt von der Theorie zur Politik uberzugehen. Die Realităt ist fast immer zu komplex, um Empfehlungen fur die Politik zuzulassen, die aus nur einem Modell abgeleitet worden sind, geschweige denn aus einem Vor-Modell von der hier skizzierten Art". M. Olson: "Das Prinzip der fiskalischen Gleichheit", a.a.O., S. 75.

Vgl. hierzu: R. Peffokoven: "Finanzausgleich I: Wirtschaftstheoretische Grundlagen", in Handwörterbuch der Wirtschaftswissenschaft, 1980, S. 613.

G. Tullock: "Főderalismus: Zum Problem der optimalen Kollektivgröße", in G. Kirsch, "Foderalismus", 1977, S. 27. 
bietet kein Argument für den Föderalismus, da es keine Basis "... for diriving an upper bound on the size of political juristdiction" ${ }^{50}$ ist. Ursache für diesen Effekt ist die Annahme, daß zentrale Entscheidungsträger das Effizienzkriterium stets erfüllen können und wollen. ${ }^{51} \mathrm{Daß}$ diese Annahme nicht haltbar ist, zeigen die politischen Steuerungsdefizite, die zentralistisch strukturierte Systeme aufweisen.

Die andere Strategie versucht, die externen Effekte auf dem Wege der Verhandlung zwischen den verschiedenen Ebenen zu internalisieren. Ziel dieser Verhandlung ist es, die Aufgaben- und Finanzierungskompetenz gemeinsam zu tragen. Die Folgen einer Verhandlungslösung zeigt Scharpf in seiner detaillierten Analyse der deutschen Politikverflechtung auf. ${ }^{52}$ Ein System der Politikverflechtung bildet Entscheidungsstrukturen aus, die "...eine massive Restriktion für die im Verflechtungssystem realisierbaren Steuerungsprogramme..." ${ }^{53}$ darstellen. Bei bestimmten Problemtypen erhöht "...die systematische Beschränkung des verfügbaren Steuerungsinstrumentariums die Wahrscheinlichkeit von 'objektiven' Steuerungsdefiziten...". ${ }^{54}$ Die starke Ausweitung von Verflechtungsstrukturen hat nicht die erhofften Effizienzgewinne gebracht, sondern vielmehr eine Lähmung des politischen Systems. ${ }^{55}$ Trotz massiver Unzufriedenheit mit den vorhandenen Strukturen ist weder eine weitere Integration noch eine Desintegration politisch durchsetzbar. Neben den Steuerungsdefiziten tendieren verflochtene Systeme zu einer Verlagerung von Kompetenzen auf die zentrale Ebene. Die Handlungsspielräume der dezentralen Einheiten nehmen mit zunehmender und fortdauernder Verflechtung immer mehr $a b$, die Verantwortung für die getroffenen Entscheidungen verschwimmt, eine Kontrolle der Entscheidungen durch den Wähler wird fast unmöglich. Bezogen auf die Regionalpolitik schlußfolgert daher Ulrich van Suntum: "Damit aber wird eines der Grundanliegen des Föderalismus, nämlich die Verhinderung einer 'Monopolisierung der Zuständigkeiten' tendenziell unterlaufen: Mischfinanzierung und Gemeinschaftsaufgaben sind keine Begrenzung der Staatsmacht, sondern ihre Bündelung."

50 J.M. Buchanan, G. Brennan: "The Power to Tax - Analytical foundations of a fiscal constitution", 1980, S. 174.

Buchannan und Brennan schreiben: "The normative advise proffered by the theory is presumably directed toward the benovelent despotism that will implement the efficiency criteria.": "The Power to Tax", a.a.O., S. 175.

Vgl. hierzu: F.W. Scharpf, B. Reissert, F. Schnabel: "Politikverflechtung: Theorie und Empirie des kooperativen Föderalismus in der Bundesrepublik", 1976.

53 F.W. Scharpf, B. Reissert, F. Schnabel: "Politikverflechtung", a.a.O., S. 66.

54 F.W. Scharpf, B. Reissert, F. Schnabel: "Politikverflechtung", a.a.O., S. 66.

Scharpf nennt dieses Phănomen „Politikverflechtungsfalle“. 
Das Prinzip der fiskalischen Äquivalenz vernachlässigt die regionalen Präferenzen, die jedoch aus wohlfahrtsökonomischer Sicht von elementarer Bedeutung für die effiziente Allokation der Ressourcen sind. Die Bereitstellung eines öffentlichen Gutes auf zentraler Ebene kann zu Wohlfahrtsverlusten führen, da die regionalen Präferenzen bei der Allokation nur einen geringen Einfluß haben. In kleinen Einheiten hingegen ist die Übereinstimmung zwischen dem angebotenen Bündel öffentlicher Güter und der Nachfrage, d.h. den Präferenzen der Einwohner, in der Regel größer. Die Mißachtung der regionalen Präferenzen führt also zu einer ineffizienten Allokation öffentlicher Güter und somit zu Wohlfahrtsverlusten.

Die Zuordnung von Kompetenzen in einem föderalen System muß sich daher an beiden Kriterien orientieren. Es müssen sowohl die Kosten der Mißachtung der regionalen Präferenzen als auch die Kosten der Vernachlässigung des Prinzips der fiskalischen Äquivalenz berücksichtigt werden. Bei einer dezentralisierten Bereitstellung eines öffentlichen Gutes sind die Kosten der Vernachlässigung der regionalen Präferenzen gering, die Kosten der Mißachtung des Prinzips der fiskalischen Äquivalenz können dagegen hoch sein. Die Beurteilung der Zuweisung von Aufgaben- und Finanzierungskompetenzen im föderalen Staat ist somit eine Abwägung zwischen zwei in ihrer Wirkung gegenläufigen Prinzipien.

Versteht man die Berücksichtigung der regionalen Präferenzen als Korrektiv, kann es dazu kommen, daß öffentliche Güter unterhalb der gemäß der fiskalischen Äquivalenz optimalen Ebene angeboten werden. ${ }^{57}$ Neben das Prinzip der fiskalischen Äquivalenz muß also das Kriterium der bestmöglichen Übereinstimmung mit den regionalen Präferenzen treten. Es ist jedoch nach wie vor notwendig, diese Abwägung für jedes einzelne öffentliche Gut vorzunehmen.

Abschließend sei noch darauf verwiesen, daß auch der Stand der Regionaltheorie bei der Zuordnung der regionalpolitischen Kompetenzen berücksichtigt werden sollte. Da es keine schlüssige theoretische Fundierung für regionalpolitisches Handeln gibt, erscheint es nicht empfehlenswert, die diesbezüglichen Kompetenzen zu zentralisieren. Die ohnehin vorhandenen Informationsprobleme sind auf der zentralen Ebene noch größer. Ferner würde der notwendige Suchprozeß nach neuen Ansätzen und Maßnahmen erheblich erschwert. Da nur ein Akteur handelt, gibt es nur eine geringe Anzahl von Versuchen. Regionalpolitische Kompetenzen müßten vielmehr dezentralisiert sein. Auch auf dezentraler Ebene ist das Informationsproblem nicht grundsätzlich gelöst, jedoch ist es im Vergleich zu zentralisierten Entscheidungskompetenzen entschärft. Ferner gibt es die Chance für eine größere Vielfalt regionalpolitischer Ansätze und Maßnahmen.

Vgl. hierzu: G. Tullock: "Föderalismus: Zum Problem der optimalen Kollektivgröße", a.a.O. sowie Pennock: "Federal and Unitary Government-Disharmony and Frustration", in "Behaiviournal Science, IV (April 1959), S. 147-157. 


\subsection{Ordnungspolitische Regionalpolitik}

Eine Volkswirtschaft ist ein dynamisches System, die Wirtschaftssubjekte müssen ständig auf Datenänderungen reagieren. Für die Zuordnung von Kompetenzen in einem föderalen System scheinen die statischen Ansätze des fiscal federalism nicht ausreichend. Eine dynamische Betrachtung, die Veränderungen der öffentlichen Leistung, der Reichweite des Nutzens oder der Präferenzen berücksichtigt, ist nicht möglich. Veränderte Rahmenbedingungen, neue wissenschaftliche Erkenntnisse oder technische Innovationen können einen hohen Anpassungsbedarf bei öffentlichen Leistungen bewirken. So muß die Polizei als Garant der inneren Sicherheit ständig auf neue Formen von Gewalt und Verbrechen reagieren. Staatliche Förderprogramme sind nur so gut, wie sie den herrschenden Bedingungen angepaßt sind. Änderungen der wirtschaftlichen Rahmenbedingungen müssen zu einer Veränderung der Programmstruktur führen.

Die Leistungsfähigkeit eines politischen Systems hängt daher von der Fähigkeit ab, neue Problemlösungen zu entwickeln und auszuprobieren. Das System muß in der Lage sein, Innovationen im Bereich der öffentlichen Leistungen zu erzeugen. Im Bereich der privaten Güter ist der Wettbewerb die entscheidende Triebfeder für Innovationen. Ältere Wettbewerbsmodelle, die von der Preistheorie beeinflußt sind, stellen nicht den Wettbewerbsprozeß, sondern sein Ergebnis in den Mittelpunkt. Ziel der Arbeiten ist es, die Bedingungen zu formulieren, die für ein Gleichgewicht erfüllt sein müssen. ${ }^{58}$ Wettbewerb kann demnach nur zu einem Gleichgewicht führen, wenn u.a. eine hohe Anzahl von Marktteilnehmern vorhanden ist, die Produkte homogen sind und vollständige Transparenz herrscht. ${ }^{59}$ Das Erkenntnisinteresse der dynamischen Wettbewerbsmodelle richtet sich auf den Prozeß des Wettbewerbs und die Voraussetzungen, die hierfür notwendig sind. Es zeigt sich, daß die Bedingungen, die für ein Gleichgewicht erfüllt sein müssen, den Wettbewerbsprozeß behindern. Das dynamische Wettbewerbskonzept kann als Basis für die Entwicklung einer ordnungspolitischen Regionalpolitik genutzt werden, die den einzelnen Ebenen des föderalen Systems bestimmte Kompetenzen zuweist. Um dies zu entwickeln, wird zunächst das Wettbewerbskonzept Hayeks dargestellt. Anschließend wird dieses Modell auf die Beziehung zwischen regionalen staatlichen Einheiten übertragen.

Hierzu gehorren das Leitbild der Marktform der vollstăndigen Konkurrenz und das Modell der vollstăndigen Konkurrenz. Vgl. hierzu Hartmut Berg: "Wettbewerbspolitik", in Vahlens Kompendium der Wirtschaftstheorie und Wirtschaftspolitik, Munchen 1990, S. 235-291/ Ernst Heuß: "Wettbewerb", in Handworterbuch der Wirtschaftswissenschaft, Göttingen 1980.

Vgl. hierzu Ernst Heuß: "Wettbewerb", a.a.O., S. 679. 


\subsubsection{Wettbewerb als Entdeckungsverfahren}

Das dynamische Wettbewerbskonzept Hayeks basiert auf seinem wirtschafts- und gesellschaftspolitischen Ordnungskonzept. Es gibt zwei Arten von Ordnungen, die gewachsene oder spontane Ordnung und die gemachte Ordnung, die von Hayek auch Organisation nennt. Die spontane Ordnung hat keinen konkreten Zweck, sie ist nicht bewußt vom Menschen erschaffen, vielmehr bildet sie den Rahmen für die in ihr handelnden Subjekte. Sie ist, so schreibt Hayek, ,... stets eine Anpassung an die große Anzahl von besonderen Tatsachen, die in ihrer Gesamtheit niemandem bekannt sind. “60 Eine Ordnung kann immer dann entstehen, wenn die handelnden Subjekte bestimmten Regeln unterworfen sind. Das heißt nicht, ,.... $\mathrm{da} ß$ die verschiedenen Personen in ähnlichen Umständen genau das gleiche tun."61 Es bedeutet nur, daß ihre Reaktion auf bestimmte Ereignisse bezüglich bestimmter abstrakter Aspekte ähnlich sein muß.

Im Gegensatz zur spontanen Ordnung verfolgt die Organisation bewußte Ziele und Zwecke. Sie reagiert auf die in ihrem Umfeld relevanten Tatsachen und paßt sich Veränderungen der Bedingungen entweder an oder geht unter. Organisationen spielen jedoch auch eine wichtige Funktion bei der Entstehung und Sicherung einer Ordnung. Die Regeln, die zur Entstehung einer Ordnung notwendig sind, entspringen drei Quellen. ${ }^{62}$

a. Die Subjekte nehmen ihre Umgebung in gleicher Art wahr

b. Regeln werden spontan befolgt, da sie Teil kultureller Überlieferung sind

c. Regeln werden auf Grund von Zwang befolgt

Die dritte Form der Regeln wird durch öffentliche Organisationen, d.h. durch die Regierung gesetzt und durchgesetzt. Eine spontane Ordnung bietet den Rahmen für das Handeln von Individuen und Organisationen. Sie ist aber gleichfalls auf die bewußte Regelsetzung durch die Regierung angewiesen. Ordnungsbildende Regeln im Sinne Hayeks sind die „rules of just conduct“, die allgemeinverbindlich sind. ${ }^{63}$ Der Regierung kommt die wichtige Aufgabe zu, diese Regeln zu definieren und die notwendigen Institutionen für ihre Durchsetzung bereitzustellen.

60 F.A. von Hayek: „Recht, Gesetzgebung und Freiheit: Band 1: Regeln und Ordnung“, a.a.O., S. 62.

61 F.A. von Hayek: „Recht, Gesetzgebung und Freiheit: Band 1: Regeln und Ordnung“, a.a.O., S. 67.

62 Vgl. F.A. von Hayek: „Recht, Gesetzgebung und Freiheit: Band 1: Regeln und Ordnung“, a.a.O., S. 68.

63 Vgl. hierzu F.A. von Hayek: „Recht, Gesetzgebung und Freiheit: Band 1: Regeln und Ordnung“, a.a.O., S. 178. 
Diesem Konzept des regelgebundenen Handelns folgt auch die Wettbewerbskonzeption Hayeks. Wettbewerb, so sagt Hayek, "... ist seiner Natur nach ein dynamischer Prozeß..." ${ }^{64}$, der mit dem Konzept des Gleichgewichts nicht beschrieben werden kann. Wettbewerbsgleichgewicht ist ein unglücklicher Ausdruck, "... because such an equilibrium presupposes that the facts have already all been discovered and competition therefore has ceased." ${ }^{65}$ Hayek setzt dem Konzept des Gleichgewichts das Konzept der Ordnung gegenüber. Eine Wettbewerbsordnung kann zu unterschiedlichen Graden erreicht sein, sie kann sich einem theoretisch bestimmten Optimum annähern, und sie kann während eines Veränderungsprozesses erhalten bleiben. ${ }^{66}$

Wettbewerb wird als „discovery procedure“ verstanden. Das Entdeckungsverfahren setzt nicht - wie das klassische Modell - bei im Markt vorhandenen Informationen an, die lediglich optimal genutzt werden müssen. Im Zentrum steht die Möglichkeit, neue Informationen, neue Verfahren, neue Produkte zu entdekken, die dann zu bisher nicht gekannten Problemlösungen beitragen. Hayek sagt: "Competition is valuable only because, and so far, its results are unpredictable and on the whole different from those which anyone has, or could have, deliberately aimed at. ${ }^{167}$ Dieses Verständnis des Wettbewerbs bringt allerdings methodologische Probleme mit sich, da es nicht möglich ist, diese Theorie empirisch zu testen. ${ }^{68}$ Hayek sieht in einem historischen Ansatz die einzige Möglichkeit, die Überlegenheit des Wettbewerbs zu beweisen. Die Fähigkeit der Gesellschaft als Ganzes, Chancen wahrzunehmen, Ziele zu erreichen und auf Änderungen zu reagieren, bestimmt den Erfolg der Gesellschaft und kann somit ein Indikator für die Wirksamkeit einer Wettbewerbsordnung sein. ${ }^{69}$

Auch für die organisatorische Struktur eines Staates lassen sich aus diesen grundlegenden Konzepten der Ordnung und der Wettbewerbsordnung Schlußfolgerungen ziehen. Die sich daraus ableitenden Empfehlungen sind von dem Bestreben geprägt, eine regelgebundene Ordnung zu sichern, die Freiraum für den Wettbe-

64 F.A. von Hayek: "Der Sinn des Wettbewerbs", in Individualismus und wirtschaftliche Ordnung, Salzburg 1976, S. 125.

F.A. von Hayek: "Competition as a Discovery Procedure", in New Studies in Philosophy, Politics, Economics and the History of Ideas, London 1978, S. 184.

Vgl. hierzu F.A. von Hayek: "Competition as a Discovery Procedure", a.a.O., S 184.

67 F.A. von Hayek: "Competition as a Discovery Procedure", a.a.O., S. 180.

68 Hayek beschreibt dieses Problem sehr plastisch: "If we do not know the facts we hope to discover by means of competition, we can never ascertain how effective it has been in discovering those facts that might be discovered." Competiton as a Discovery Procedure, a.a.O. S 180 .

Vgl. F.A. von Hayek: "Competition as a Discovery Procedure", a.a.O., S 180. 
werbsprozeß läßt. Hayek beschreibt dieses Grundproblem so: "The relevant distinction is ... between conditions, on the one hand, in which alternative ways based on different views or practices may be tried and conditions, on the other, in which one angency has the exclusive right and the power to prevent others from trying." ${ }^{70}$ Somit ist die staatliche Struktur zu wählen, die einer Machtkonzentration Vorschub leistet, die zur Abschaffung einer regelgebundenen Ordnung Mißbraucht werden kann. Allerdings bedarf es einer zentralen Regierung, die die Funktion der Setzung und Durchsetzung von Regeln wahrnehmen kann. Unabhängig von der staatlichen Ebene richtet sich Hayek gegen "... all exclusive, privileged, monopolistic organizations, against the use of coercion to prevent others from trying to do better." ${ }^{71}$ Ein Staat kann nur dann Wachstum und Wohlstand sichern bzw. mehren, wenn er den einzelnen Einheiten Freiraum für eigene Entscheidungen läßt. ${ }^{72}$ Wesentliche Voraussetzung einer Wettbewerbsordnung in einem System ist somit die Dezentralisierung der Kompetenzen. Hayek versteht diese Konzeption bewußt als ein Modell für die Beziehungen zwischen zentralen und dezentralen Einheiten. ${ }^{73}$

Diese Grundgedanken finden sich auch in Hayeks Standpunkt zur Dezentralisierung von öffentlichen Leistungen wieder. Unter der Bedingung der strikten Regelbindung könnten die meisten Dienstleitungsaktivitäten ,... dann in der Tat vorteilhafterweise an regionale oder lokale Behörden delegiert werden..." “74 Strikte Regelbindung bedeutet, daß keine Diskriminierung innerhalb der regelsetzenden Gebietskörperschaft erfolgen kann und damit „Subventionswettläufe“ unterbunden werden. Diese Lösung ist auch deshalb vorteilhaft, weil die Bereitstellung öffentlicher Leistungen durch regionale und lokale Einheiten generell die erstbeste Alternative für ein privates Leistungsangebot ist. Sie hat, so Hayek, viele Vorteile der privaten Leistungserbringung und ,... fewer dangers of the coercive action of government." 675

F.A. von Hayek: "The constitution of Liberty", London 1960, S. 37.

F.A. von Hayek: "The constitution of Liberty", a.a.O., S. 37.

Von Hayek sieht die Abwesenheit von umfassender Kontrolle als Voraussetzung fur die Entwicklung einer Gesellschaft: "There can be little doubt that man owes some of the greatest successes in the past to the fact that he has not been able to control social life.", aus "The Constitution of liberty", .a.a.O., S. 38.

In dem Vorwort zu dem Buch "The constitution of Liberty" sagt von Hayek sehr deutlich, daß er ein Ideal darstellen will: "My aim is to picture an ideal, to show how it can be achieved, and to explain what it realization would mean in practice."

74 F.A. von Hayek: „Recht, Gesetzgebung und Freiheit: Band 1: Regeln und Ordnung“, a.a.O., S. 182. 
Hayek interpretiert den regelgebundenen Wettbewerb zwischen lokalen und regionalen Einheiten als ein Wettbewerb um die Wohnbevölkerung. Wenn sie weder die Freizügigkeit beschränken noch in der Besteuerung diskriminieren können, würden sie sich darauf konzentrieren, ,... diejenigen anzulocken, die in ihrer besonderen Situation den größten Beitrag zu dem gemeinsamen Produkt leisten könnten. ${ }^{676}$ Die Verlagerung des Management der meisten Dienstleistungen auf die kommunale Ebene ,... würde wahrscheinlich zu einer Wiederbelebung kommunalen Geistes führen, der weitgehend durch die Zentralisierung erstickt worden ist. “77

\subsubsection{Wettbewerb zwischen regionalen Einheiten - ein ordnungspolitisches Konzept der Regionalpolitik}

Um ein ordnungspolitisches Konzept der Regionalpolitik entwickeln zu können, muß es Kriterien oder Prinzipien für eine Zuordnung der regionalpolitischen Kompetenzen in der bestehenden föderalen Struktur geben. Wie oben gezeigt, läßt sich Wettbewerb im privatwirtschaftlichen Bereich als regelgebundenenes Entdeckungsverfahren interpretieren. Nur ein dynamischer Ansatz wird der Komplexität der Märkte und der Geschwindigkeit, mit der sie sich verändern, gerecht. Auch die Anbieter öffentlicher Leistungen sind von diesen Prozessen betroffen, da die Rahmenbedingungen, unter denen sie agieren, sowie die Anforderungen an ihre Leistungen einem ständigen Wandel unterworfen sind. Daher erscheint es durchaus als sinnvoll, das dynamische Konzept des Wettbewerbs auf die Beziehung zwischen unterschiedlichen Anbietern öffentlicher Leistungen zu übertragen. So läßt sich ein ordnungspolitisches Konzept der Regionalpolitik entwickeln. Ferner können Prinzipien für die Zuordnung regionalpolitischer Kompetenzen ableitet werden.

Eine ordnungspolitische Regionalpolitik geht davon aus, daß regionale wirtschaftliche Entwicklungsprozesse nicht bewußt plan- und steuerbar sind. Die Anbieter staatlicher Leistungen stehen in diesem Modell im Wettbewerb um mobile Faktoren. Sie sind an die Regeln des Wettbewerbs gebunden und können diese nicht ändern. Parameter in diesem Wettbewerbsprozeß sind die Faktoren, die die Attraktivität einer Region für mobile Produktionsfaktoren determiniert. Dies sind u.a. Art, Qualität, Preis und Struktur der öffentlichen Leistungen.

Im Rahmen einer dynamischen Wettbewerbsordnung können die regionalen Einheiten im Sinne der Hayekschen Terminologie als Organisationen verstanden

76 F.A. von Hayek: „Recht, Gesetzgebung und Freiheit: Band 1: Regeln und Ordnung“, a.a.O., S. 198.

77 F.A. von Hayek: „Recht, Gesetzgebung und Freiheit: Band 1: Regeln und Ordnung“, a.a.O., S. 198/199. 
werden, die durch bewußtes ziel- und zweckgerichtetes Verhalten gekennzeichnet sind. Im Rahmen unserer existierenden föderalen Struktur sind dies die Länder sowie die kommunalen Gebietskörperschaften. Mit ihrem öffentlichen Leistungsangebot stehen die regionalen Einheiten untereinander im Wettbewerb um mobile Produktionsfaktoren. Qualität, Preis und Struktur der öffentlichen Leistungen sind wichtige Faktoren der Attraktivität für Kapital, Humanressourcen und technologisches Know-How. Im Rahmen eines als Entdeckungsverfahren verstandenen Wettbewerbs können Innovationen im Bereich der öffentlichen Leistungen generiert werden. Strukturen, Finanzierungsmodelle, Art und Qualität der Leistung, die Zusammensetzung des Leiustungsbündels und viele andere Parameter können Gegenstand eines so verstandenen Wettbewerbsprozesses sein.

Damit ein solcher Prozeß möglich ist und die positiven Effekte des Wettbewerbs realisiert werden können, muß eine adäquate Ordnung geschaffen werden. Dies führt direkt zu der Frage, welche regionalpolitischen Kompetenzen auf welcher Ebene zuzuordnen sind. Die zuzuordnenden Kompetenzen unterteilen sich in die reinen Ziel-Mittel Entscheidungen und die Festlegung allgemeiner Regeln für den Wettbewerb. Die Setzung und Durchsetzung von Regeln muß auf zentraler Ebene geschehen. Die späteren Analysen werden zeigen, daß dies auf europäischer Ebene angesiedelt werden muß. Im Rahmen dieser Regeln kann dann ein Wettbewerb zwischen den regionalen Einheiten entstehen. Die reinen Mittel-Zweck Entscheidungen, also die Entwicklung und Implementation von Programmen, muß in der Verantwortung der regionalen Einheiten liegen. Die Dezentralisierung dieser Kompetenzen ist daher Grundvoraussetzung für die Bereitstellung öffentlicher Güter im Rahmen einer Wettbewerbsordnung.

Bisher haben wir eine eindeutige Zuordnung der Kompetenz zur Regelsetzung auf zentraler Ebene. Bei einem mehrstufigen föderalen Aufbau, wie wir ihn in Deutschland haben, bleibt die Frage offen, auf welcher Ebene die Entscheidung über Programme und deren Implementation angesiedelt werden soll. Die Zuordnung sollte, orientiert am Prinzip der fiskalischen Äquivalenz unter Berücksichtigung der regionalen Präferenzen, vorgenommen werden. Wie die Analyse gezeigt hat, führen diese Prinzipien nicht $\mathrm{zu}$ eindeutigen Zuordnungen. Im Sinne des Wettbewerbskonzeptes muß die Zuordnung im Zweifel so weit unten wie möglich erfolgen. Dieses Prinzip steht durchaus im Einklang mit der fiskalischen Äquivalenz. Wie oben dargestellt, müßte theoretisch für jede öffentliche Leistung ein eigenes öffentliches Kollektiv gebildet werden. Diese Forderung läßt sich in der Realität nicht durchsetzen. Anzahl und Struktur der föderativen Ebenen ist in der Regel nicht bzw. nur unter Einsatz hoher Transaktionskosten veränderbar. ${ }^{78}$ Die einzelnen Ebenen können sich jedoch jederzeit zu Zweckverbänden zusammen-

Im Rahmen von Kreisgebietsreformen bzw. der Fusion von Ländern wird lediglich die Anzahl der Mitglieder einer Ebene verăndert. 
schließen, um gemeinsam ein öffentliches Gut anzubieten. Durch dieses Verfahren können sich maßgeschneiderte Kollektive gründen, die eine optimale Internalisierung der externen Effekte sicherstellen. ${ }^{79} \mathrm{Da}$ es grundsätzlich ein Kündigungsrecht gibt, können die Mitglieder das Kollektiv auch wieder verlassen. ${ }^{80}$ Durch dieses Konzept ist der Wettbewerb zwischen den föderativen Einheiten bzw. zwischen verschiedenen Zweckverbänden sichergestellt.

Ein ordnungspolitisches und dezentralisiertes Modell der Regionalpolitik hat Vorteile gegenüber einem zentralisierten konstruktivistischen Ansatz. Es ist einem zentralisierten System bezüglich der Anpassungsfähigkeit und Innovationsfähigkeit überlegen. In dezentralisierten Systemen gibt es eine große Vielfalt von Wissen, Fähigkeiten und Begabungen. Dieses Humankapital ist ungleichmäßig über die verschiedenen Akteure in den Regionen verstreut. Daher gilt auf der regionalen Ebene, da $§$ "... individuals with very different and even quite extraordinary backgrounds can be selected as government agents, and the peculiar stimuli provided by each local environment offers most favourable conditions for the emergence of new ideas and discoveries. ${ }^{81}$ In einem zentralisierten System ist diese Vielfalt stark eingeschränkt, die fehlenden Handlungsspielräume der zentral berufenen lokalen Repräsentanten sind keine erfolgversprechende Ausgangsbasis für die Entdeckung neuer Problemlösungen.

Ein zentrales Argument für Wettbewerb zwischen regionalen Einheiten ist die Verfügbarkeit von Informationen. Die wohlfahrtsökonomischen Vorteile der Zentralisierung lassen sich nur realisieren, wenn die Zentrale in der Lage ist, die gleiche Menge und Vielfalt an Informationen zu gewinnen, zu speichern und zu bearbeiten, die in einem dezentralisierten System vorhanden ist. In der Realität ist die Informationsgewinnungs- und verarbeitungsfähigkeit einer zentralen Entscheidungseinheit der bedeutendste Engpaßfaktor. Die Zentrale wird nie die Vielfalt und Menge an Informationen gewinnen, speichern und bearbeiten können, die in den regionalen Einheiten vorhanden ist. ${ }^{82}$

79 Dieses Konzept des funktionalen Foderalismus wird von Straubhaar vorgeschlagen. Vgl. hierzu: "Zur optimalen Größe eines integrierten Wirtschaftsraumes: Das Konzept des funktionalen Föderalismus", Diskussionsbeiträge zur Wirtschaftspolitik, Institut fur Wirtschaftspolitik, Universităt der Bundeswehr Hamburg, 1993.

80 Diese "exit option" wird allerdings durch die Hobhe der sunk cost eingeschränkt. Der Austritt einer Gemeinde aus einem Abwasserzweckverband ist daher eher unwahrscheinlich.

81 Martti Vihanto: "Competition between Local Governments",in: Journal of Institutional and Theoretical Economics (JITE), 148(1992)., S. 417.

Vgl. hierzu Martti Vihanto: "Competition Between Local Governments", a.a.O., S. 415418. 


\subsection{Zusammenfassung}

Zunächst wurde in diesem Kapitel geprüft, auf welcher Ebene regionalpolitische Kompetenzen angesiedelt werden sollten. Dies geschah unter der Prämisse, daß die praktizierte Regionalpolitik einem konstruktivistischen Politikansatz folgt, der versucht, wirtschaftliche Entwicklung bewußt zu planen und zu steuern. Die $\mathrm{Zu}$ ordnung der Zuständigkeiten im Rahmen der bestehenden föderalen Struktur kann die Defizite dieser Regionalpolitik noch verschärfen. Daher war zu prüfen, ob eine zentrale oder dezentrale Zuordnung vorteilhaft ist.

Die Analyse hat gezeigt, daß die in der Literatur vorhandenen Kriterien zu keiner eindeutigen Antwort führen. Das Prinzip der fiskalischen Äquivalenz orientiert sich an der Internalisierung der externen Effekte öffentlicher Leistungen. Die Größe der regionalen Einheit, die eine öffentliche Leistung bereitstellt, muß sich somit an der Reichweite des Nutzens dieser Leistung orientieren. Auch wenn keine hundertprozentige Internalisierung der externen Effekte angestrebt wird, tendiert dieses Prinzip letztendlich zu einer Zentralisierung von Kompetenzen. Es haben sich zwei Strategien herausgebildet, um externe Effekte öffentlicher Leistungen zu internalisieren. Entweder wählt man die Ebene, auf der keine Externalitäten mehr vorhanden sind. Dies führt in einer stark verflochtenen Volkswirtschaft im Ergebnis zur Zentralisierung. Oder man wählt den Weg der Politikverflechtung. Hierbei wird die Entscheidungs- und Finanzierungskompetenz über mehrere Ebenen eines politischen Systems aufgeteilt. Auch diese Strategie führt letztendlich zu einer Zentralisierung von Kompetenzen.

Als weiteres Kriterium für die Zuordnung von Kompetenzen sollte die Berücksichtigung der regionalen Präferenzen herangezogen werden. Dieses aus der Wohlfahrtsökonomie stammende Kriterium verlangt, daß sich die Allokation öffentlicher Güter an den regionalen Präferenzen orientieren sollte. Je weiter die Kompetenzen dezentralisiert sind, desto mehr können die regionalen Präferenzen Berücksichtigung finden.

Im Ergebnis ist die Zuordnungsentscheidung eine Abwägung zwischen den beiden Prinzipien. Die Kosten der Vernachlässigung der regionalen Präferenzen sind gegen den Nutzen der Beachtung des Prinzips der fiskalischen Äquivalenz abzuwägen. Im Zweifel sollte einer dezentralisierten Zuordnung der Vorzug gegeben werden.

Diese statische Betrachtung der Zuordnungsentscheidung ist nicht ausreichend. Öffentliche Leistungen werden im Rahmen einer sich dynamisch entwickelnden Volkswirtschaft erbracht. Wie die privaten Wirtschaftssubjekte stehen auch die öffentlichen Einheiten mit ihrem Leistungsangebot im Wettbewerb um mobile Produktionsfaktoren. Für den privatwirtschaftlichen Bereich läßt sich dieser Prozeß mit dem dynamischen Wettbewerbsmodell Hayeks beschreiben. Dieses Modell einer regelgebundenen Wettbewerbsordnung, das Wettbewerb als Entdekkungsverfahren versteht, in dem in einem offenen Prozeß neue, bis dahin nicht 
gekannte Tatsachen entdeckt werden, läßt sich auf die Beziehung zwischen regionalen Einheiten übertragen. Indem wir dies tun, entwickeln wir ein ordnungspolitisches Konzept der Regionalpolitik, das als Basis für die weitere Analyse dient.

Die regionalen Einheiten werden im Sinne der Hayekschen Terminologie als Organisationen interpretiert. Organisationen sind bewußt geschaffene Strukturen, die konkreten Zwecken und Zielen dienen. Sie handeln im Rahmen einer Ordnung, deren Strukturen nicht bewußt geplant oder geschaffen worden sind. Eine Ordnung entsteht vielmehr aus drei Quellen. Zum einen reagieren die Wirtschaftssubjekte auf bestimmte Tatsachen in ähnlicher Weise. Zum anderen prägen die herrschenden Sitten und Gebräuche eine Ordnung. Neben diesen beiden Quellen muß es jedoch noch eine Instanz geben, die Regeln setzt und durchsetzt. Diese Rolle kommt einer Organisation zu und zwar der Regierung.

Aus diesem Konzept lassen sich dann auch Schlußfolgerungen für die Zuordnung der Kompetenzen vornehmen. In einem ordnungspolitischen Konzept der Regionalpolitik obliegt der zentralen Instanz die Regelsetzung- und durchsetzung. Die regelgebundenen Ziel-Mittel Entscheidungen obliegen den regionalen Einheiten. 


\section{Regionalpolitik in Mecklenburg-Vorpommern}

Regionalpolitik wird in der Europäischen Gemeinschaft (EG) und in Deutschland im Rahmen eines verflochtenen Systems geplant und implementiert. Alle Ebenen des föderalen Systems Europa, von der Gemeinschaft über den Bund und die Länder bis hin zu den kommunalen Gebietskörperschaften sind an diesem Prozeß inhaltlich und finanziell beteiligt. Ausgehend von einem ordnungspolitischen Konzept der Regionalpolitik soll die so praktizierte Regionalpolitik untersucht werden. Die Analyse setzt ganz bewußt auf der Ebene der Bundesländer ein, da dies die Ebene im föderalen Staat ist, die im Rahmen eines ordnungspolitischen Konzepts der Regionalpolitik konkrete regelgebundene Maßnahmen beschließt und implementiert. Da Regionalpolitik in Europa und Deutschland in einem verflochtenen System durchgeführt wird, müssen auch die regionalpolitisch relevanten Aktivitäten der Gemeinschaft und des Bundes sowie deren Auswirkungen auf die Landesebene untersucht werden. Ausgehend von der theoretischen Analyse stehen folgende Fragen im Mittelpunkt:

a. Welches wirtschaftspolitische Konzept liegt der Regionalpolitik zugrunde?

b. Welche Regionaltheorien werden zur Fundierung der Programme herangezogen und gelingt es, in sich schlüssige Ziele und Instrumente zu definieren? Wenn dies nicht gelingt, warum nicht?

c. Wie werden die regionalpolitischen Handlungs- und Finanzierungskompetenzen zugeordnet? Folgt die Zuordnung dem Prinzip der fiskalischen Äquivalenz und werden regionaler Präferenzen berücksichtigt?

d. Sind Ansätze einer ordnungspolitischen Regionalpolitik erkennbar, gibt es einen regelgebundenen Wettbewerb regionaler Einheiten? Welche Gründe führen zu Abweichungen von dem ordnungspolitischen Konzept der Regionalpolitik?

Der Gang der Untersuchung orientiert sich an dem idealtypischen Ablauf eines politischen Planungsprozesses, der mit einer Analyse der Ausgangslage beginnt. In Kapitel 4 wird die Wirtschaftsstruktur Mecklenburg-Vorpommerns dargestellt und die Chancen und Risiken des Wirtschaftsraumes verdeutlicht. Der nächste Schritt eines idealtypischen Planungsprozesses ist die wirtschaftspolitische Zielsetzung. Da Regionalpolitik in einem verflochtenen System geplant und implementiert wird, werden auf allen beteiligten Ebenen Ziele definiert. In Kapitel 5 wird das sich daraus ergebende regionalpolitische Zielsystem im Lichte folgender Fragen untersucht: a. Gibt es ein konsistentes Zielsystem? b. Welche wirtschaftspolitischen Konzeptionen liegen den Zielen zu Grunde?

Zur Umsetzung dieses Zielsystems werden Instrumente entwickelt. Für die Regionalpolitik sind dies die unterschiedlichen Förderprogramme, die in Kapitel 6 dargestellt und analysiert werden. Es soll geprüft werden, welche Regionaltheo- 
rien Anwendung finden, wie die Kompetenzzuordnung erfolgt und welches wirtschaftspolitische Konzept den Programmen zu Grunde liegt.

Den Abschluß eines Planungsprozesses bzw. den Startpunkt eines neuen Prozesses bildet die Evaluation der Programme. Es wird geprüft, ob die Ziele erreicht wurden, ob die Instrumente umsetzbar waren und wenn nein, welche Veränderungen notwendig sind. Eine echte Wirkungsanalyse ist für die Regionalpolitik nicht darstellbar, jedoch kann in Kapitel 7 gezeigt werden, ob die Vorgaben der Programme im Rahmen der Implementation eingehalten wurden.

$\mathrm{Da}$ eine ordnungspolitische Regionalpolitik nur dann realisierbar ist, wenn die Länder eigene Programme entwickeln und zur erfolgreichen Umsetzung dieser Programme bereit und in der Lage sind, werden abschließend die landeseigenen regionalpolitischen Programme, die nicht im Rahmen des EFRE und der GA geplant und umgesetzt werden, betrachtet. Zunächst wird geprüft, welche Freiräume das Land für eigene Aktivitäten hat. In einem zweiten Schritt wird dann dargestellt, wie es diese ausfüllt.

\section{Wirtschaftsstruktur Mecklenburg-Vorpommerns}

Die regional- und strukturpolitischen Herausforderungen der neuen Länder werden maßgeblich durch die veränderten wirtschaftlichen, politischen und rechtlichen Rahmenbedingungen bestimmt. Alle neuen Länder haben zum Beispiel Probleme bezüglich der Wettbewerbsfähigkeit der Industrie und der Kompensation für die weggebrochenen Ostmärkte. In allen Ländern führt der Prozeß der Regelung der Eigentumsfragen zu mehr oder weniger starken Behinderungen.

Es gibt allerdings Unterschiede in der Betroffenheit durch diese überregionalen Faktoren, die im wesentlichen in den bestehenden Wirtschaftsstrukturen, der Lage und den sich daraus ergebenen Entwicklungspotentialen begründet sind.

Mecklenburg-Vorpommern ist mit 80 Einwohnern je qkm das am dünnsten besiedelte Land der Bundesrepublik Deutschland (der Durchschnitt der Bevölkerungsdichte im Bundesgebiet liegt bei 225 Einwohner je qkm). Lediglich in den sechs kreisfreien Städten, in denen ca. 34\% der Gesamtbevölkerung leben, liegt die Bevölkerungsdichte über 1000 Einwohnern je qkm. In den Landkreisen bewegt sie sich zwischen 31 und 104 Einwohnern je qkm. ${ }^{83}$ Eine derart dünne Besiedlung stellt die Regional- und Strukturpolitik vor besondere Herausforderungen.

Auch die Wirtschaftsstruktur des Landes ist keine besonders gute Ausgangsbasis für eine dynamische wirtschaftliche Entwicklung. Im Vergleich zu den anderen ostdeutschen Flächenländern hat Mecklenburg-Vorpommern den höchsten Be-

Die Zahlen stammen aus dem Statistischen Jahrbuch 1993 für MecklenburgVorpommern. 
schäftigtenanteil in der Land- und Forstwirtschaft und im Dienstleistungsbereich, den niedrigsten im produzierenden Gewerbe. Während bei modernen Volkswirtschaften allgemein ein hoher Dienstleistungsanteil als Anzeichen für eine gesunde Wirtschaftsstruktur gilt, hat dieses Urteil für Mecklenburg-Vorpommern keine Gültigkeit.

Tabelle 1

\begin{tabular}{|l|c|c|c|}
\hline \multicolumn{3}{|c|}{$\begin{array}{c}\text { Strukturunterschiede in Ostdeutschland } \\
\text { (Erwerbstätigenanteile 1989 nach Sektoren) }\end{array}$} \\
\hline & $\begin{array}{c}\text { Land und } \\
\text { Forstwirtschaft }\end{array}$ & $\begin{array}{c}\text { Produzierendes } \\
\text { Gewerbe }\end{array}$ & $\begin{array}{c}\text { Dienstleistungs- } \\
\text { bereich }\end{array}$ \\
\hline Brandenburg & $15,30 \%$ & $42,20 \%$ & $42,50 \%$ \\
\hline Meckl.-Vorp. & $\mathbf{1 9 , 6 0 \%}$ & $\mathbf{3 2 , 1 0 \%}$ & $\mathbf{4 8 , 3 0 \%}$ \\
\hline Sachsen & $7,30 \%$ & $52,80 \%$ & $40,00 \%$ \\
\hline Sachsen-Anhalt & $12,20 \%$ & $46,50 \%$ & $41,30 \%$ \\
\hline Thüringen & $10,20 \%$ & $50,80 \%$ & $39,10 \%$ \\
\hline Berlin (O) & $1,10 \%$ & $35,10 \%$ & $63,90 \%$ \\
\hline Quelle: Bundesministerium für Raumordnung, Bauwesen und Städtebau (Hrsg.): \\
Raumordnungsbericht 1991 der Bundesregierung, Bonn 1991, Anhang 8, S. 192.
\end{tabular}

Eine nähere Analyse der Zahlen zeigt, daß ein überproportional hoher Anteil der Beschäftigen des Dienstleistungsbereichs für den Staat tätig ist. Nimmt man das Flächenland Schleswig-Holstein als Maßstab, dann müßte der Anteil der beim Staat beschäftigten Personen auf ca. $12 \%$ zurückgehen. Die Beschäftigungsstruktur und die Entwicklung der Beschäftigung im verarbeitenden Gewerbe läßt weitere Strukturdefizite erkennen. Fast $25 \%$ der Beschäftigten des verarbeitenden Gewerbes sind direkt im Schiffbau tätig. Obwohl es mit $42 \%$ in diesem Bereich nicht zu einem überdurchschnittlichen Beschäftigungsabbau kam, ist die Abhängigkeit des verarbeitenden Gewerbes vom Schiffbau bedenklich. Der vorläufige Erhalt der Arbeitsplätze war nur mit erheblichen Subventionen möglich. Wie die internationale Entwicklung im Schiffbau zeigt, ist jedoch in diesem Bereich mit einem weiteren Arbeitsplatzabbau zu rechnen. Es ist fraglich, ob der Schiffbau in Mecklenburg-Vorpommern in dieser Größe ohne nennenswerte 
Subventionen zu halten sein wird. ${ }^{84}$ Die Abhängigkeit vom Schiffbau ist für die wirtschaftliche Entwicklung des Landes ein großes Risiko.

Tabelle 2

\begin{tabular}{|c|c|c|}
\hline \multicolumn{3}{|c|}{$\begin{array}{l}\text { Beschäftigtenanteile nach Wirtschaftssektoren in } \\
\text { Mecklenburg-Vorpommern und Schleswig-Holstein } 1990 \\
\text { (in Prozent der Gesamtbeschäftigten) }\end{array}$} \\
\hline & $\begin{array}{l}\text { Mecklenburg- } \\
\text { Vorpommern }\end{array}$ & $\begin{array}{l}\text { Schleswig- } \\
\text { Holstein }\end{array}$ \\
\hline Land und Forstwirtschaft & $15,00 \%$ & $8,70 \%$ \\
\hline Energie- und Wasservers., Bergbau & $2,00 \%$ & $1,40 \%$ \\
\hline Verarbeitendes Gewerbe & $20,40 \%$ & $27,30 \%$ \\
\hline Baugewerbe & $8,00 \%$ & $6,90 \%$ \\
\hline Handel und Verkehr & $19,70 \%$ & $19,40 \%$ \\
\hline Private Dienstleistungen & $7,60 \%$ & $24,70 \%$ \\
\hline Sonst. Dienstleistungen (Staat) & $27,30 \%$ & $11,60 \%$ \\
\hline
\end{tabular}

Sehr positiv stellt sich hingegen das Ernährungsgewerbe dar. Hier kam es nur zu unterdurchschnittlichen Arbeitsplatzverlusten. Es wird erwartet, daß in diesem Bereich mittelfristig neue Arbeitsplätze geschaffen werden können. ${ }^{85}$ Die Bedeutung dieser beiden Wirtschaftszweige wird noch deutlicher, wenn man die Anteile am Umsatz betrachtet. Im Jahr 1994 entfallen auf diese beiden Wirtschaftszweige $57,1 \%$ des Umsatzes im verarbeitenden Gewerbe. Das verarbeitende Gewerbe wird somit von einem dynamischen Wirtschaftszweig und einem stagnierenden bzw. schrumpfenden Wirtschaftszweig dominiert. Ziel der Regionalund Strukturpolitik ist es daher, die Einseitigkeit der Wirtschaftsstruktur zu verringern.

Eine umfassende Analyse der Situation und der Perspektiven des Schiffbaus in Mecklenburg-Vorpommern hat die Prognos AG fur das Wirtschaftsministerium vorgenommen. Sie entwickelt zwei mögliche Anpassungspfade, die allerdings beide einen hohen Beihilfenbedarf haben. Prognos AG: "Instrumente und Informationssysteme fur die Wirtschaftspolitik des Landes Mecklenburg-Vorpommern", 1992, S. 122-148.

Vgl. hierzu: Prognos AG: "Instrumente und Informationssysteme für die Wirtschaftspolitik des Landes Mecklenburg-Vorpommern", 1992, S. 104. 
Tabelle 3

\section{Beschäftigtenabbau ausgewählter Zweige des verarbeitenden Gewerbes (Mecklenburg-Vorpommern 1991-1992)}

\begin{tabular}{|l|c|c|c|}
\hline & $\begin{array}{c}\text { Beschäftigte } \\
\text { Dez. 92 }\end{array}$ & $\begin{array}{c}\text { Abbau von } \\
\mathbf{0 1 . 9 1} \text { bis } \\
\mathbf{1 2 . 9 2}\end{array}$ & $\begin{array}{c}\text { Abbau } \\
\text { in \%* }\end{array}$ \\
\hline $\begin{array}{l}\text { Verarbeitendes Gewerbe } \\
\text { Davon: }\end{array}$ & 54.446 & -65.160 & $43 \%$ \\
\hline Schiffbau & 13.365 & -16.514 & $42 \%$ \\
\hline Ernährungsgew., Tabakverarbeitung & 12.044 & -12.703 & $39 \%$ \\
\hline Maschinenbau & 5.875 & -12.692 & $56 \%$ \\
\hline Elektrotechnik & 6.132 & -5.281 & $33 \%$ \\
\hline Straßenfahrzeugbau, Reparatur v. Kfz & 2.762 & -2.481 & $43 \%$ \\
\hline Gewinnung u. Verarb. v. Steinen u. Erden & 1.747 & -1.410 & $24 \%$ \\
\hline Bekleidungsgewerbe & 500 & -2.418 & $78 \%$ \\
\hline * Abbau in Prozent der Beschäftigten am 01.01.1991 \\
$\begin{array}{l}\text { Quelle: Statistisches Landesamt Mecklenburg-Vorpommern: Statistische Monatshefte } \\
\text { 6/1993, Schwerin 1993, S 14 }\end{array}$ \\
\hline
\end{tabular}

Neben den allgemeinen regional- und strukturpolitischen Herausforderungen, die für alle neuen Länder gleich sind, gibt es landesspezifische Herausforderungen, die aus der regionalen Wirtschafts- und Bevölkerungsstruktur resultieren. Regional- und strukturpolitische Programme müssen daher auf die speziellen landestypischen Strukturen abgestimmt werden. Dies ist eine originäre landespolitische Aufgabe, die durch den Bund oder die Gemeinschaft nicht geleistet werden kann. Somit unterstreicht diese knappe Wirtschaftsstrukturanalyse die Bedeutung der richtigen Zuordnung von regional- und strukturpolitischen Kompetenzen. Das Land kann dieser Aufgabe nur gerecht werden, wenn es eigenständig Ziele setzen, Instrumente definieren und implementieren kann. Diese Eigenständigkeit muß sich in Entscheidungskompetenzen und Finanzierungskompetenzen widerspiegeln. 
Tabelle 4

\begin{tabular}{|c|c|c|}
\hline \multicolumn{3}{|c|}{$\begin{array}{l}\text { Umsätze des verarbeitenden Gewerbes } \\
\text { (Mecklenburg-Vorpommern } 1990 \text { und } 1993\end{array}$} \\
\hline & $\begin{array}{l}\text { Anteil am Gesamtum- } \\
\text { satz 2. Halbjahr } 1990\end{array}$ & $\begin{array}{c}\text { Anteil am } \\
\text { Gesamtumsatz } 1994\end{array}$ \\
\hline Steine und Erden & $1,1 \%$ & $9,3 \%$ \\
\hline Maschinenbau & $10,3 \%$ & $5,3 \%$ \\
\hline Straßenfahrzeugbau & $4,1 \%$ & $4,9 \%$ \\
\hline Schiffbau & $22,3 \%$ & $21,6 \%$ \\
\hline Elektrotechnik & $8,7 \%$ & $5,9 \%$ \\
\hline Holzbearbeitung & $0,6 \%$ & $1,1 \%$ \\
\hline Holzverarbeitung & $2,8 \%$ & $3,3 \%$ \\
\hline Ernährungsgewerbe & $39,8 \%$ & $35,5 \%$ \\
\hline Sonstige & $10,3 \%$ & $13,1 \%$ \\
\hline
\end{tabular}

\section{Regionalpolitik in Mecklenburg Vorpommern: Die Ziele}

Die Analyse beginnt mit der Zielsetzung für die Regionalpolitik in Mecklenburg-Vorpommern. Basierend auf Zielen lassen sich die notwendigen Maßnahmen bzw. Instrumente und Mittel definieren. Ferner sind Ziele Maßstab für eine Erfolgskontrolle, die dann Rückschlüsse auf notwendige Veränderungen der Maßnahmen und Instrumente geben kann. Die Zieldefinition ist daher die wichtigste Aufgabe in einem Planungsprozeß. Damit die Ziele ihre Funktion erfüllen können, müssen sie bestimmten Kriterien genügen. Die Ziele sollten sich logisch in eine Zielhierarchie ordnen lassen. Zumindestens auf den unteren Ebenen einer Zielhierarchie sollte der Grad der Operationalisierung der Ziele eine Kontrolle ermöglichen.

Für die Regionalpolitik sollte es ein Zielsystem geben, das diesen Anforderungen genügt. Da Regionalpolitik in der EG und in Deutschland in einem verflochtenen System durchgefuihrt wird, definiert jede Ebene regionalpolitische Ziele. Die Zielvorgaben der jeweils höheren Ebene haben Bindungswirkung für die nachgeordneten Ebenen. Die Länder sind daher bereits in der Phase der Zielsetzung in ihren Entscheidungen an Vorgaben der Kommission und des Bundes gebunden. Entsprechend der generellen Fragestellung dieses Abschnittes sind folgende Fragen zu prüfen: 
a. Ist aus der Zielsetzung bereits ein wirtschaftspolitisches Konzept erkennbar?

b. Welche regionalpolitischen Theorien liegen den Zielen zugrunde, gelingt es, eine konsistente Zielhierarchie zu entwickeln? Wenn nein, warum nicht?

c. Haben die Zielsetzungen Auswirkungen auf die Kompetenzzuordnung und wenn ja welche? Warum erfolgt die Kompetenzzuordnung so und nicht anders?

d. Sind die Ziele mit einem ordnungspolitischen Konzept der Regionalpolitik vereinbar?

Die Untersuchung geht von den allgemeinen regionalpolitischen Zielen $\mathrm{zu}$ den konkreten programmatischen Zielen. Daher werden zunächst die Verfassungsebene sowie allgemeine gesetzliche Bestimmungen des Bundes und des Landes betrachtet. Konkretisiert bzw. umgesetzt werden diese Vorgaben durch die regionalpolitischen Ziele der drei Ebenen. Diese sind Gegenstand des zweiten Teils der Untersuchung.

\subsection{Grundlegende regionalpolitische Ziele in Verfassungen und Gesetzen}

\subsubsection{Zielvorgaben des Grundgesetzes und der Landesverfassung}

Als erstes gilt es, die Vorgaben der Verfassungsebene zu betrachten, die grundsätzliche Kompetenzzuweisung und allgemein gehaltene Zielbestimmungen beinhalten.

Im Grundgesetz sind im wesentlichen zwei Bestimmungen für die Regionalpolitik relevant. In Art 91a (Gemeinschaftsaufgaben von Bund und Ländern) wird dem Bund ein Mitwirkungsrecht bei Länderaufgaben eingeräumt, soweit diese Aufgaben für die Gesamtheit bedeutsam sind und eine Beteiligung des Bundes zur "Verbesserung der Lebensverhältnisse" erforderlich ist. Desweiteren hat Art 106 Abs. 3 Nr. 2 Bedeutung. Der Bund wird verpflichtet, die "Einheitlichkeit der Lebensverhältnisse im Bundesgebiet" zu wahren. ${ }^{86}$

Die Landesverfassung von Mecklenburg-Vorpommern setzt andere Schwerpunkte. Der Artikel 17 (1) schreibt als Staatsziel fest: "Das Land trägt zur Erhaltung und Schaffung von Arbeitsplätzen bei. Es sichert im Rahmen des gesamtwirtschaftlichen Gleichgewichts einen hohen Beschäftigungsstand." Staatsziel ist die Schaffung von Arbeitsplätzen. Die Verfassung macht keine Aussagen über die Verteilung der Arbeitsplätze, sie fordert keinen Ausgleich zwischen den Regionen des Landes, wie es in Art 106. Abs. 3 Nr. 2 GG geschieht.

Diese Formulierung bezieht sich eigentlich auf die Verteilung der Umsatzsteuer zwischen Bund und Ländern. Sie wird jedoch als Begründung für die Ausgleichsfunktion der Regionalpolitik herangezogen. 
Die Kompetenzzuordnung des Grundgesetzes ist nicht eindeutig. Die verfassungsmäßige Zulässigkeit von Gemeinschaftsaufgaben ermöglicht dem Bund grundsätzlich ein Steuerungspotential in Politikbereichen, die ansonsten in die alleinige Zuständigkeit der Länder fallen würden. Ob der Bund diese Aufgabe dann durch Setzung allgemeiner Regeln oder durch konkrete Maßnahmenprogramme umsetzt, bleibt zunächst offen. Die Verfassung ist somit bezüglich der wirtschaftspolitischen Konzeption neutral. Sie läßt eine konstruktivistisch planende und steuernde Politik genauso zu, wie eine ordnungspolitische Konzeption. Dieser Befund gilt auch für die Landesverfassung, die bezüglich der wirtschaftspolitischen Konzepte, die zur Verfolgung des Staatsziel eingesetzt werden, keine Festlegungen trifft. Im Rahmen einer ordnungspolitischen Konzeption der Regionalpolitik müßte der Bund die Regeln für den Wettbewerb der regionalen Einheit setzen. Dem Land obläge es dann unter Beachtung dieser Regeln, regionalpolitische Programme zu definieren und zu implementieren.

\subsubsection{Das Raumordnungsgesetz}

Eine erste Konkretisierung der regionalpolitischen Ziele des Bundes findet sich im Raumordnungsgesetz (ROG) ${ }^{87}$. Leitvorstellung der Raumordnung ist die freie Entfaltung der Persönlichkeit, der Schutz der natürlichen Lebens-grundlagen, die Offenhaltung von Gestaltungsmöglichkeiten in der Raumnutzung sowie gleichwertige Lebensbedingungen der Menschen in allen Teilräumen. ${ }^{88}$

Die Grundsätze der Raumordnung konkretisieren das allgemeine Leitbild. In den alten Bundesländern sollen die Lebensbedingungen in Regionen verbessert werden, in denen diese "... in ihrer Gesamtheit im Verhältnis zum Bundesdurchschnitt wesentlich zurückgeblieben sind..." ${ }^{89}$. In den neuen Bundesländern sollen "... Lebensbedingungen sowie eine Wirtschafts- und Sozialstruktur geschaffen werden, die denen im übrigen Bundesgebiet gleichwertig sind."10

Das ROG sieht sowohl die Sicherung von Verdichtungsräumen ${ }^{91}$ als auch den Erhalt ländlicher Räume und ihrer Siedlungsstruktur vor, d.h. Abwanderungen sollen verhindert werden. Ein Wandel der Raumstruktur, eine Wanderung von Bevölkerung bzw. von Produktionsfaktoren ist nach den Bestimmungen des

Neufassung des Raumordnungsgesetzes vom 28.04.1993 (BGBl. I S. 630). Die Neufassung wurde auf Grund des Investitionserleichterungs- und Wohnbaulandgesetzes vom 22.04 .93 notwendig.

Vgl. §1 (1) Nr. 1-4 ROG.

$\S 2$ (1) Nr. 3 ROG.

$\S 2$ (1) Nr. 4 ROG.

91

Vgl. §2 (1) Nr. 5 ROG. 
ROG nicht gewollt. Der durch die Raumplanung - also politisch - bestimmte status quo soll erhalten bzw. verfestigt werden.

Die Grundsätze gelten unmittelbar für die Landesplanung in den Bundesländern $^{92}$, die die Verwirklichung des Leitbildes und der Grundsätze sicherstellen soll. ${ }^{93}$ Die Länder können weitere Grundsätze aufstellen, diese dürfen allerdings nicht im Widerspruch zu denen des ROG stehen.

Das Ziel der gleichwertigen Lebensbedingungen wird in dem Gesetz nicht näher definiert, ferner werden keine Indikatoren benannt, mit denen dieser Zustand gemessen werden könnte.

Die objektive Beurteilung von Lebensbedingungen ist von einer zentralen Instanz nicht leistbar. Man könnte versuchen, anhand von vielfältigen Infrastrukturkennziffern die Frage der Gleichwertigkeit der Lebensbedingungen von $\mathrm{Hu}-$ sum in Nordfriesland und Hannover zu klären. Dieser Versuch ist aber allein schon deshalb problematisch, weil es sich um eine Wertung von Lebensbedingungen handelt. ${ }^{94}$ Hierbei ist die Bewertung einer zentralen Instanz anhand eines einheitlichen Indikators von nachgeordneter Bedeutung. Von entscheidender Bedeutung sind vielmehr die Präferenzen der Einwohner, diese müßten der eigentliche Maßstab für die Bewertung von Lebensbedingungen sein. Doch weder in dem Leitbild, noch in den Grundsätzen der Raumordnung spielen die Präferenzen der Einwohner eine Rolle. Zwar verpflichtet das ROG die Länder zur Beteiligung der Kommunen an der Regionalplanung, jedoch sind die Kommunen genauso an die Vorgaben des ROG gebunden wie die Länder. ${ }^{95}$

Das Raumordnungsgesetz ist bezüglich der wirtschaftspolitischen Konzeption nicht mehr neutral. Die Leitvorstellungen ließen sich noch als allgemeine Regeln für regionalpolitisches Handeln regionaler Einheiten interpretieren. Jedoch zeigt die weitere Analyse, daß der Bund selber konkrete Vorstellungen über die Raumnutzung, die Siedlungsstruktur und die Lebensbedingungen in den Regionen entwickeln und durchsetzen will. Bereits auf dieser noch sehr wenig konkreten Zielebene deutet sich eine konstruktivistisch planende Politikkonzeption an.

Vgl. §3 (2) S. 1 ROG.

Vgl. §4 (3) ROG.

Die Lebensbedingungen sollen ja nicht gleich sein, sondern gleichwertig. Damit stellt sich die Frage, wieviel die Năhe zur Nordsee, die grunen Wiesen im Umland der Stadt und die relative Abgeschiedenheit im Vergleich zu zahlreichen Theatern, umfangreichen Einkaufsmöglichkeiten und einem breiten Schul- und Bildungsangebot wert sind.

Năheres regelt $§ 5$ ROG. Die Lănder werden verpflichtet, die Kommunen bzw. die regionalen Planungsverbände zu beteiligen. Ihnen bleibt allerdings ein Regelungsspielraum bezüglich der Organisation der Raumplanung. Diese kann entweder durch das Land, bzw. durch regionale Planungsgemeinschaften erfolgen. 
Ferner zeigen sich die erwartbaren Schwierigkeiten einer derartigen Politikkonzeption. Die Formulierung einer widerspruchsfreien Zielhierarchie gelingt selbst auf dieser sehr allgemeinen Ebene nicht. Das ROG will auf der einen Seite die bestehenden Strukturen bewahren (Verhinderung von Abwanderung, Sicherung des ländlichen Raumes und der Verdichtungsräume) und auf der anderen Seite Wachstums- und Anpassungsprozesse ermöglichen.

\subsubsection{Raumordnung in Mecklenburg-Vorpommern}

Als nächste relevante Ebene muß die Raumordnung in MecklenburgVorpommern analysiert werden. Sie wurde nach den Vorgaben des Bundesraumordungsgesetzes aufgebaut. Die gesetzliche Grundlage legte das Land 1992 mit dem Landesplanungsgesetz. Das erste Landesraumordnungsprogramm wurde im Juli 1993 in Kraft gesetzt.

\section{Das Landesplanungsgesetz}

Im Landesplanungsgesetz (LPIG) für Mecklenburg-Vorpommern finden sich erwartungsgemäß ähnliche Akzente wie im ROG, sowie weitergehende konkretere Zielvorgaben. Wichtigster Grundsatz der Landesplanung ist die Schaffung gleichwertiger Lebensbedingungen in allen Teilräumen des Landes sowie die Vermeidung von Abwanderungen. ${ }^{96}$ Ferner soll "die Wirtschaft nachhaltig gestärkt und der Strukturwandel so unterstützt werden, daß die Wirtschafts- und Leistungskraft möglichst rasch bundesweites Niveau erreicht und ausreichend viele Arbeitsplätze geschaffen sowie gesichert werden." ${ }^{\text {"97 }}$

Der Aus- bzw. Neubau von Verkehrsanlagen und Kommunikationsnetzen soll so betrieben werden, daß eine leistungsfähige Verbindung der einzelnen Landesteile sowie eine Kompensation der Randlage Mecklenburg-Vorpommerns innerhalb der Bundesrepublik möglich ist. Mecklenburg-Vorpommern will sich als Brücke zum Norden Europas profilieren.

Im Rahmen eines zentralörtlichen Netzes soll eine Infrastruktur geschaffen werden, die zwar einen Strukturwandel der Wirtschaft ermöglicht bzw. befördert, jedoch Abwanderungen verhindert.

Auch im LPIG Mecklenburg-Vorpommerns findet sich der Dualismus von Wandel und Beharrung. Auf der einen Seite wird der dringend notwendige Strukturwandel gefordert und unterstützt, auf der anderen Seite sollen Veränderungen in der bestehenden Bevölkerungsverteilung verhindert werden. Ob dieser Dualismus einer Entfaltung der dringend notwendigen Wachstumskräfte förderlich ist, bleibt zu prüfen.

Vgl. § 2 (1) Nr. 1 LPIG vom 31.03.1992, GVOBl. M-V S. 242. 
Das Ziel der Förderung von Arbeitsplätzen hat keine regionale Komponente. Es sollen möglichst viele neue Arbeitsplätze geschaffen werden. $\mathrm{Ob}$ und inwieweit dieses Ziel mit der Verwirklichung der nicht näher definierten gleichwertigen Lebensverhältnisse in Einklang zu bringen ist, bleibt offen. Durch die Einführung eines Achsenkonzeptes sind die Räume für wirtschaftliche Aktivitäten vorgegeben. Unternehmer, die außerhalb dieser Bereiche investieren wollen, werden nur wenig Aussicht auf die Genehmigung ihrer Tätigkeit haben.

Auch hier zeigen sich die erwartbaren Zielkonflikte, die entstehen, wenn wirtschaftliche Entwicklungsprozesse basierend auf unzureichenden theoretischen Konzeptionen gesteuert werden sollen. Ob gleichwertige Lebensverhältnisse und eine Stabilisierung des ländlichen Raumes vereinbar sind mit einem Achsenkonzept, das bewußt differenziert und nur in bestimmten Teilräumen wirtschaftliche Entwicklung zuläßt, bleibt fraglich.

\section{Das Landesraumordnungsprogramm}

Die nächste Stufe der Zieldefinition und Operationalisierung stellt das Landesraumordnungsprogramm ${ }^{98}$ dar. Diese Untersuchung beschränkt sich auf die Ziele, die den Bereich Wirtschaft betreffen. Zunächst werden drei allgemeine Ziele formuliert. Sie lassen sich wie folgt zusammenfassen: ${ }^{99}$

1. Schaffung guter Standortbedingungen zur Ansiedlung von Gewerbebetrieben sowie Ausbau der Infrastruktur mit Schwerpunkt in den zentralen Orten

2. Entwicklung des vorhandenen und ausbaufähigen Potentials der einzelnen Regionen

3. Berücksichtigung der örtlichen und regionalen Gegebenheiten

Dem produzierenden Gewerbe sollen bedarfsorientiert Industrie- und Gewerbeflächen bereitgestellt werden. Standorte für Industrie und Gewerbe sollen in der Regel die zentralen Orte sein. In Ausnahmefällen können auch "... Siedlungen im Zuge überregionaler und innerregionaler Achsen in Betracht ..." kommen. ${ }^{100}$

Das Land hält an den Werftstandorten fest, unterstützt die Werften bei der sozialverträgliche Umstrukturierung und der Erlangung internationaler Wettbewerbsfähigkeit. Die Nahrungsmittelindustrie hat für Mecklenburg-Vorpommern eine besondere Bedeutung. In zentralen Orten sollen wettbewerbsfähige Betriebe angesiedelt werden.

Der Wirtschaftsminister des Landes Mecklenburg-Vorpommern: "Erstes Landesraumordnungsprogramm Mecklenburg-Vorpommern", 16.07.1993.

Vgl. "Erstes Landesraumordnungsprogramm Mecklenburg-Vorpommern", a.a.O., S. 36.

Vgl. "Erstes Landesraumordnungsprogramm Mecklenburg-Vorpommern", a.a.O., S. 44. 
Auch im Bereich Handwerk, Handel und Dienstleistungen sollen moderne und wettbewerbsfähige Betriebe angesiedelt werden. Bevorzugte Standorte sind auch hier die zentralen Orte. Handwerk und Dienstleistungen sollen bedarfsorientiert unterstützt werden, wobei insbesondere bei Dienstleistungen auf ein räumlich ausgewogenes Angebot hinzuwirken ist.

Die Forderung nach Konzentration von Infrastruktur, produzierendem Gewerbe und Dienstleistungen in zentralen Orten deutet auf den ersten Blick auf eine starke Orientierung der Raumordnung an dem Konzept der zentralen Orte, dem Konzept der Wachstumspole sowie den Ansätzen der neuen Wachstumstheorie hin. Gemäß dieser Konzepte ist eine Konzentration der Maßnahmen auf zentrale Orte erforderlich, da eine Bündelung verschiedener Unternehmen und eine ausreichende Infrastrukturausstattung Grundvoraussetzung für Wachstum und Ausstrahlungseffekte eines Zentrums sind. ${ }^{101}$ Das Raumordnungsprogramm fordert, ganz im Einklang mit den theoretischen Konzepten, gute Standortbedingungen für Gewerbebetriebe, damit ausreichend Arbeitsplätze geschaffen werden können. Die zentralen Orte sollen "... Schwerpunkte der wirtschaftlichen Entwicklung bilden ..." ${ }^{102}$, in verstärktem Maße soll dies für Ober- und Mittelzentren gelten.

Die Zahl der zentralen Orte ist allerdings beträchtlich. Neben den vier Oberzentren (mehr als 100.000 Einwohner) gibt es 11 Mittelzentren (mehr als 15000 Einwohner) und 8 Mittelzentren mit Teilfunktionen (mehr als 10000 Einwohner). Ferner gehören zu den zentralen Orten noch die Unterzentren (mehr als 4000 Einwohner) sowie die ländlichen Zentralorte (mehr als 1000 Einwohner).

Aus den Ausführungen ist ganz eindeutig eine planende und steuernde Konzeption der Regionalpolitik abzulesen. Es sollen bedarfsorientiert Gebiete zugewiesen werden und ganz bestimmte Sektoren gefördert werden. Grundsätzlich wäre dies mit einer ordnungspolitischen Regionalpolitik vereinbar, da das Land die Ebene ist, die im Rahmen der Regeln konkrete ziel- und zweckgerichtete Maßnahmen beschließen kann. Doch auch hier zeigen sich die Schwächen der theoretischen Fundierung einer solchen Politik. Es gibt die gleichen Zielkonflikte, wie sie für das Raumordnungsgsetz und das Landesplanungsgesetz schon festgestellt worden. Ferner wird hier die Spannung zwischen ökonomischer und politischer Rationalität deutlich. Die ökonomische Theorie verlangt nach einer Konzentration der Anstrengungen. Die Zahl der zentralen Orte ist jedoch so groß, daß es zweifelhaft ist, ob eine effektive Konzentration von wirtschaftlicher

101 In neueren Untersuchungen wird eine Bevőlkerungsdichte von 150 Einwohnern je qkm als notwendig angesehen. Dies erreicht Mecklenburg-Vorpommern - auf Kreisebene betrachtet - nur in den Oberzentren.

Vgl. "Erstes Landesraumordnungsprogramm Mecklenburg-Vorpommern", a.a.O., S. 36. 
Tätigkeit und Infrastruktur wirklich gewollt ist. Die Formulierungen lassen sowohl eine Konzentration der Infrastruktur- und Wirtschaftsförderung in den Ober- und Mittelzentren zu als auch eine breite Streuung der Mittel über alle zentralen Orte. Es ist aus Sicht der politischen Ökonomie dieser Verteilungsentscheidung eben nicht unerheblich, wieviel Menschen in den Genuß der politischen Förderung gelangen. Die politische Rationalität spricht daher ganz deutlich für eine breite Streuung der Anstrengungen und Mittel.

\subsubsection{Prozeß der Landesplanung: Top down statt bottom up}

An dieser Stelle ist es angebracht, die Zuordnung der Kompetenz, regionalpolitisch relevante Ziele verbindlich festzulegen, näher zu analysieren.

Adressat der regionalpolitischen Ziele ist immer die untere Ebene des föderalen Systems. Diese hat im Bereich der Infrastrukturinvestitionen und der Ansiedlung von Gewerbe eine herausragende Bedeutung. Auf die gleiche Ebene zielt der Begriff des endogenen Potentials, das Basis für jedwede regionale Entwicklung sein soll bzw. muß. Im Prozeß der Raumordnung spielt die kommunale Ebene jedoch nur eine untergeordnete Rolle. Der Raumordnungsprozeß wird zentral gesteuert. Die Vorlage des Raumordnungsprogramms wird von der Landesplanungsbehörde erarbeitet. Hierbei sind u.a. die Landkreise und kreisfreien Städte zu beteiligen, die wiederum die kreisangehörigen Gemeinden beteiligen. ${ }^{103}$ Der so entwickelte Plan wird den Beteiligten mit der Bitte um Stellungnahme zugeleitet und letztendlich von der Landesregierung festgestellt. ${ }^{104}$ Die Landesplanung ist nicht die Summe der auf regionalen Präferenzen und Zielen beruhenden Einzelplanungen, sondern das Ergebnis eines Abstimmungsprozesses, in dem die Betroffenen zwar das Recht zur Meinungsäußerung haben, jedoch weder direkt noch indirekt Entscheidungskompetenz haben. ${ }^{105}$

Grundvoraussetzung für eine ordnungspolitische Regionalpolitik ist die Befugnis der regionalen Einheiten, bewußt Ziele zu setzen. Dies ist im Raumordnungsprozeß nur eingeschränkt möglich, da durch die zentrale Planung Funktionszuweisungen und Einschränkungen vorgenommen werden, die die Möglichkeit der eigenständigen Zielsetzung der regionalen Einheiten stark reduzieren. Ferner ist es aus wohlfahrtstheoretischen Überlegungen sinnvoll, die regionalen Präferenzen bei der Zielsetzung stärker zu berücksichtigen. Die Möglichkeiten, regionale Präferenzen in diesem Planungsprozeß zur Geltung zu bringen, sind sehr eingeschränkt. Der Raumordnungsprozeß in Mecklenburg-Vorpommern ist ein stark zentralisiertes Verfahren, die Kompetenzen zur Planung und Entscheidung der

Vgl. $§ 7$ (1) LPlG.

Weder ist eine Abstimmung im Landtag noch eine theoretisch denkbare Beteiligung der Kreise an der Feststellung des Raumordnungsprogramms vorgesehen.
} 
Landesplanungsprogramme sind überwiegend bei der Landesregierung konzentriert.

Um den Präferenzen der Regionen bei der Funktionszuweisung und der Zuweisung von Entwicklungsmöglichkeiten ein höheres Gewicht zu geben und auf der Ebene der Raumplanung einen Standortwettbewerb zu ermöglichen, müßte der Prozeß umgekehrt werden. Aus dem top-down-approach müßte ein bottom-upapproach werden. Das Raumordnungsprogramm des Landes müßte die Summe der regionalen Planungen sein. Die regionalen Planungsverbände, die es bereits gibt, könnten auf ihrer Ebene die Planungen der Gemeinden und Kreise abstimmen. Auch hier müßte jedoch der Ausgangspunkt aller Überlegungen die an den Präferenzen der Einwohner orientierten Vorgaben der Gemeinden sein. Der Planungsverband hat dann primär eine moderierende bzw. ausgleichende Funktion zu übernehmen. Ferner kann ihm die Planungshoheit für bestimmte öffentliche Güter übertragen werden, soweit eine Verletzung des Prinzips der fiskalischen Äquivalenz vorliegt und eine Internalisierung der externen Effekte über andere Strukturen - z.B. Zweckverbände - nicht gewährleistet werden kann.

Lediglich in den Fällen, in denen übergeordnete Interessen nicht ausreichend Berücksichtigung finden, kann und muß das Land die Planungshoheit behalten. Dies können nur solche Aufgaben sein, bei denen eine regionalisierte Planung zu einer Verletzung des Prinzips der fiskalischen Äquivalenz führen würde und eine anderweitige Internalisierung der externen Effekte nicht möglich ist.

Eine Reform des Raumordnungsprozesses scheitert an zwei Faktoren. Zum einen ist das Vertrauen und der Glaube an konstruktivistische Politikkonzeptionen ungebrochen. Das Land als „benevolent despot“ weiß besser als die Kreise, Städte und Gemeinden, welche regionalpolitischen Ziele und Maßnahmen notwendig sind. Regionalisierte Kompetenzen führen zu unsachgemäßen Entscheidungen. Ferner ist es politisch sehr mühselig, einen Planungsprozeß, der von unten determiniert wird, zu steuern. Entscheidend dürften jedoch die politikökonomischen Gründe sein. Das Land, d.h. die Landesregierung, die beteiligten Ministerien, das Parlament als Institution, die einzelnen Parlamentarier und nicht zuletzt die sie entsendenden Parteien haben kein Interesse, dieses Steuerungspotential aufzugeben. Mit der Planung und Zuweisung von Funktionen an Teilräume können Wahlchancen verbessert und somit Mehrheiten gesichert werden. Ein zentralisierter Planungsprozeß schafft entsprechende Stellen in der Landesbürokratie, die den Parteien zum Ausbau bzw. zur Stabilisierung ihrer internen und externen Machtstrukturen dienen.

\subsection{Ziele der regionalen Wirtschaftspolitik}

Bisher wurden die verfassungsrechtlichen beziehungsweise die allgemeinen gesetzlichen Zielvorgaben betrachtet. In diesem Abschnitt stehen die konkreten 
wirtschaftspolitischen Ziele der verschiedenen Träger bzw. Instrumente der regionalen Wirtschaftspolitik im Vordergrund. Auf dieser Ebene der Analyse sind die konkretesten Zielbestimmungen zu erwarten. Somit wird spätestens hier die die Regionalpolitik tragende wirtschaftspolitische Konzeption deutlich. Ferner läßt sich zeigen, ob die Gemeinschaft oder der Bund Ansätze einer ordnungspolitischen Regionalpolitik haben. Schlußendlich kann geprüft werden, welche Handlungsfreiräume den Ländern im Bereich der konkreten Zielsetzung bleiben.

\subsubsection{Regionalpolitik der EG: Ziele}

In der Präambel des Vertrages über die Europäische Wirtschaftsgemeinschaft (EWGV) von 1957 bringt die Gemeinschaft ihren Willen zum Ausdruck, "... ihre Volkswirtschaften zu einigen und deren harmonische Entwicklung zu fördern, indem sie den Abstand zwischen einzelnen Gebieten und den Rückstand weniger begünstigter Gebiete ..." ${ }^{106}$ verringert. Dieser allgemeinen regionalpolitischen Zielsetzung stand allerdings keine Handlungskompetenz der Gemeinschaft gegenüber. ${ }^{107}$ Im Jahre 1975 wurde mit dem Europäischen Fonds für Regionale Entwicklung (EFRE) ein regionalpolitisches Instrument der Gemeinschaft geschaffen. ${ }^{108}$ Eine Verankerung im EWGV fand die Regionalpolitik jedoch erst 1987 mit der Verabschiedung der Einheitlichen Europäischen Akte. ${ }^{109}$

Ziel der europäischen Regionalpolitik ist, den wirtschaftlichen und sozialen $\mathrm{Zu}-$ sammenhalt (Kohäsion) der Gemeinschaft zu stärken. Die Gemeinschaft setzt sich insbesondere zum Ziel, "... den Abstand zischen den verschiedenen Regionen und den Rückstand der am wenigsten begünstigten Gebiete zu verringern."110 Ausgehend von den drei großen europäischen Zielen der Wirtschaftspolitik - Sta-

Präambel des EWG Vertrages, zitiert nach "Europarecht", Baden-Baden, 1992, S. 18.

Grundsătzlich gilt für die EWG das Prinzip der enumerativen Einzelermăchtigung. Die Gemeinschaft darf nur insoweit tătig werden, als die Verträge als primäres Gemeinschaftsrecht ihr dazu ausdrucklich die Befugnis geben.

Die Gründung des Fonds stütze sich auf Art 235 EWGV. Dieser erlaubt der Gemeinschaft in Bereichen tătig zu werden, die für die Schaffung des gemeinsamen Marktes von $\mathrm{Be}$ deutung sind.

Zu der Entwicklung der Europäischen Regionalpolitik u.a.: Helmut Neupert: "Regionale Strukturpolitik als Aufgabe der Länder: Grundlagen, Verknüpfungen, Grenzen", BadenBaden, 19../ M. Schäfers, J. Starbatty: "Das Instrumentarium der EG zur Förderung innergemeinschaftlicher Kohäsion", in 'Aus Politik und Zeitgeschichte', B28/90, 06.07.1990, S. 3-15.

Art 130 a EWGV nach Änderung durch die EEA. 
bilität, Wachstum und Verteilungsgerechtigkeit ${ }^{111}$ - ist die Verwirklichung des Binnenmarktes das primäre wachstumspolitische Programm, die Schaffung der Wirtschafts- und Währungsunion das primäre stabilitätspolitische Programm. Die Regionalpolitik konzentriert sich auf ausgleichspolitische Programme. Betrachtet man die Entwicklungsgeschichte der europäischen Regionalpolitik, so wurde sie von den nationalen Regierungen stets als Kompensation für die Folgen fortschreitender wirtschaftlicher bzw. monetärer Integration gesehen. ${ }^{112}$ Dem Verständnis der Strukturfonds als quasi vertikalen Finanzausgleich der Gemeinschaft versuchte die Kommission eine eigenständige, ziel- und programmorientierte Regionalpolitik entgegen zu stellen. Die zum Zeitpunkt des Beitritts der fünf neuen Länder gültige Verordnung sah für die drei Strukturfonds EFRE, ESF (Europäischer Sozialfonds) und EAGFL (Europäischer Ausrichtungs- und Garantiefonds für die Landwirtschaft, Abteilung Ausrichtung) fünf Ziele vor: ${ }^{113}$

Ziel 1: Förderung von Regionen mit Entwicklungsrückstand

Ziel 2: Förderung von Regionen, die von rückläufiger industrieller Entwicklung schwer betroffen sind

Ziel 3: Bekämpfung der Langzeitarbeitslosigkeit

Ziel 4: Eingliederung von Jugendlichen in das Erwerbsleben

Ziel 5: Anpassung der Agrarstruktur und Entwicklung des ländlichen Raumes

Die Verordnung ordnet den Zielen einen oder mehrere Fonds zu, die die Finanzierung der Maßnahmen übernehmen sollen, damit eine Koordinierung der einzelnen Fonds sichergestellt ist. An den fünf Zielen wird die ausgleichspolitische

An diesen Zielen orientiert sich auch ein Bericht einer Expertenkommission, die die wirtschaftlichen Folgen des Beitritts Spaniens und Portugals sowie des Binnenmarktes untersucht hat. Tommaso Padoa-Schioppa: "Effizienz, Stabilität und Verteilungsgerechtigkeit", Gabler, 1987.

Jeder Vorstoß in Richtung Währungsunion bzw. Verwirklichung des Binnenmarktes fuhrte zu Ausgleichsforderungen seitens der wirtschaftlich schwăcheren Mitgliedsstaaten. Aber auch anläßlich von Erweiterungen waren und sind derartige Kompensationen notwendig. Im Zuge der Debatte um eine mögliche Osterweiterung der EU fordern die Empfängerländer eine Aufstockung der Fördermittel als Voraussetzung für eine Zustimmung. Vgl. hierzu R. Ridinger: "Aktuelle Diskussionen zur Finanzausstattung und Reform der EG-Regionalforderung", Wirtschaftsdienst 1992/XII, S. 650 / o.V.: "Ein neuer EU-Fonds fur dünnbesiedelte Gebiete - Endspurt bei Beitrittsverhandlungen", Die Tageszeitung vom 23.01.1994.

113 Vgl. Verordnung (EWG) Nr. 2052/88 des Rates vom 24.06.1988 uber Aufgaben und Effizienz der Strukturfonds und uber die Koordinierung ihrer Interventionen untereinander sowie mit denen der Europäischen Entwicklungsbank und der anderen vorhandenen Finanzierungsinstrumente. Amtsbl. EG, Nr. L 158/9 vom 15.07.1988. 
Ausrichtung der europäischen Regionalpolitik deutlich. Die im engeren Sinne regionalpolitischen Ziele (Ziel 1,2 und 5) konzentrieren sich auf regionalpolitische Problemregionen. Neben diesen regionalpolitischen Zielen verfolgt die Kommission mit den Strukturfonds auch sozial- und arbeitsmarktpolitische Ziele, die nicht unbedingt einen regionalen Bezug haben müssen. Diese Vermischung von regionalen und funktionalen Zielen birgt die Gefahr in sich, daß die Gemeinschaft in Bereichen tätig wird, in denen sie mit ihren beschränkten Mitteln nur wenig bewirken kann. ${ }^{114}$

Die Gemeinschaft verfolgt bewußt eine ziel- und programmorientierte Regionalpolitik, um ihren Anspruch als regionalpolitscher Akteur zu untermauern. Die Politik orientiert sich an einem konstruktivistischen wirtschaftspolitischem Konzept, das die Entwicklung in den Regionen Europas gemäß den fünf Zielen steuern will. In diesem Bereich der europäischen Regionalpolitik sind keine Ansätze für eine ordnungspolitische Regionalpolitik zu finden.

Ganz deutlich lassen sich die Zentralisierungstendenzen verflochtener Systeme aufzeigen. Die Regionalpolitik der Gemeinschaft ist von Ziel und Interessendivergenzen zwischen der Kommission und dem Europäischen Parlament auf der einen Seite sowie den nationalen Regierungen und Parlamenten auf der anderen Seite geprägt. Dies wurde zuletzt in der Debatte des Europäischen Parlaments zur Reform der Strukturfondsverordnung deutlich. Das Parlament, das die Kommission stets in ihrem Bestreben nach einem stärkeren Einfluß auf die Regionalpolitik der Mitgliedsländer unterstützt hat, kritisierte die im Rat vorherrschende Tendenz zur "Renationalisierung" der Regionalpolitik. ${ }^{115}$

Die am wenigsten von nationalen Interessen bestimmte europäische Institution, die Kommission, möchte ihre eigenen regionalpolitischen Ziele und Programme in den Regionen durchsetzen. Sie ist bemüht, ihren Einfluß auf die nationalen bzw. regionalen Entscheidungsträger auszubauen. ${ }^{116}$ Hierbei ist sie allerdings auf die Kooperation mit den nationalen und regionalen Regierungen bzw. Verwaltungen angewiesen, die tendenziell ihre Unabhängigkeit wahren wollen. Im Ergebnis führt diese Ziel- und Interessendivergenz zu einer Verbürokratisierung der Regionalförderung der Gemeinschaft.

Vgl. hierzu auch Padoa-Schiopa, T: "Effizienz, Stabilităt und Verteilungsgerechtigkeit", a.a.O., S. 255.

Vgl. Hartmut Hausmann: "Reform der Strukturfonds: Vom Mauerblümchen zum wichtigsten Finanzinstrument", Das Parlament, 30.07.1993.

Der zunehmende Einfluß der Gemeinschaft wird auch im Rahmen der Beihilfenkontrolle immer deutlicher. Waren bisher Infrastrukturmaßnahmen von der Beihilfenkontrolle ausgeschlossen, dehnt die Kommission ihre Überprufung derzeit auch auf diesen Bereich aus. 


\subsubsection{Ziele der Gemeinschaftsaufgabe}

Seit 1969 ist die Verbesserung der regionalen Wirtschaftsstruktur als Gemeinschaftsaufgabe von Bund und Ländern im Grundgesetz verankert. Die GA wurde in einer Zeit eingerichtet, in der wirtschaftspolitisches Handeln durch die Annahme der Planbarkeit und Steuerbarkeit volkswirtschaftlicher Prozesse bestimmt war.

Die Gemeinschaftsaufgabe muß "... mit den Grundsätzen der allgemeinen Wirtschaftspolitik und den Zielen und Erfordernissen der Raumordnung und Landesplanung übereinstimmen." ${ }^{117}$ Die Ziele und Programme der Raumordnung und der Landesplanung sind für die GA bindend ${ }^{118}$, ihre Funktionszuweisungen und Einschränkungen begrenzen den Handlungsspielraum der regionalen Wirtschaftspolitik.

Ferner hat sie auf gesamtdeutsche Belange und auf Erfordernisse der Europäischen Gemeinschaft Rücksicht zu nehmen. Die Programme der GA, die jährlich im Rahmenplan fortgeschrieben werden, müssen gemäß Art $92 \mathrm{ff}$ EWGV durch die Kommission genehmigt werden. Die Gemeinschaft hat somit einen erheblichen Einfluß auf die Ziele und Programme der bundesdeutschen Regionalpolitik. Die bundesdeutsche Regionalpolitik verfolgt drei Ziele. Das Ausgleichsziel, das dem Leitgedanken der gleichwertigen Lebensbedingungen der Raumordnung entspricht, will interregionale Unterschiede "... hinsichtlich der Möglichkeiten zur Einkommenserzielung und der Ausstattung mit Arbeitsplätzen"119 abbauen. Das Wachstumsziel besteht in der "... Mobilisierung von Wachstumsreserven in den Problemregionen, um den Beitrag dieser Gebiete zum gesamtwirtschaftlichen Wachstum zu erhöhen". ${ }^{120}$ Das Stabilitätsziel will die strukturelle und konjunkturelle Anfälligkeit der Regionen reduzieren.

Diese drei Ziele bilden kein konsistentes Zielbündel. Zwischen den Zielen können Zielkonflikte auftreten ${ }^{121}$, deren Lösung einer klaren Setzung von Prioritäten bedarf. Weder die Raumordnung, noch die GA gehen auf diese Zielkonflikte ein

$117 \S 2$ (1) Gesetz uber die Gemeinschaftsaufgabe "Verbesserung der regionalen Wirtschaftsstruktur" vom 06.10.1969, BGBl. I, S. 1861.

Eine Voraussetzung fur eine Förderung durch die GA ist daher die Übereinstimmung des Vorhabens mit der Landesplanung.

Deutscher Bundestag: Einundzwanzigster Rahmenplan der Gemeinschaftsaufgabe "Verbesserung der regionalen Wirtschaftsstruktur", vom 13.05.1992, Drucksache 12/2599, S. 6.

Deutscher Bundestag Einundzwanzigster Rahmenplan der Gemeinschaftsaufgabe "Verbesserung der regionalen Wirtschaftsstruktur", vom 13.05.1992, a.a.O., S. 6.

Die Zielkonflikte sollen hier nicht ausfuhrlich diskutiert werden. Eine ausfuhrliche Diskussion findet sich u.a. bei U. van Suntum: "Regionalpolitik in der Marktwirtschaft", Baden-Baden 1981, S. 26-54. 
und geben auch keinen Hinweis, welchem Ziel Priorität eingeräumt werden sollte. Die Schlußfolgerung van Suntums macht dieses Defizit sehr deutlich: "Wenn aber Zielkonflikte schon auf dieser relativ allgemeinen und damit unverbindlichen Ebene geleugnet bzw. einfach ignoriert werden, so ist die konsistente Ableitung einzelner Maßnahmen aus den übergeordneten regionalpolitischen Zielen praktisch unmöglich. Damit aber würde selbst dann, wenn die einzelnen regionalpolitschen Ziele operational definiert wären - was nicht der Fall ist - die Operationalität des Zielsystems insgesamt fehlen." ${ }^{122}$ Hier zeigen sich erneut die Defizite konstruktivistischer Politikansätze. Mangels einer geschlossenen Regionaltheorie scheitert der politische Planer bereits an der Bildung eines konsistenten Zielsystems. Dies ist jedoch nur eine Ursache für diesen Mangel. Ein weiterer Grund ist in den politik-ökonomischen Rationalitäten zu suchen. Die GA will die Wirtschaftsförderung der Länder koordinieren und einen ungeordneten Förderwettlauf unterbinden. Der Bund wählt nicht den ordnungspolitischen Ansatz, der die Regelsetzung für die Förderung strikt zentralisiert hätte. Er verlagert die Wirtschaftsförderung als Gemeinschaftsaufgabe vielmehr in ein verflochtenes System. In diesem System werden die Handlungs- und Finanzierungskompetenzen von Bund und Ländern gemeinsam getragen. Dies führt dazu, daß regionalpolitische Ziele so definiert sein müssen, daß jeder der Beteiligten Anrecht auf Förderung hat.

\subsubsection{Regional- und wirtschaftspolitische Ziele des Landes Mecklenburg- Vorpommern}

Alle bisher besprochenen Zielsysteme sind für die regional- und wirtschaftspolitischen Ziele des Landes von Bedeutung. Innerhalb dieses Rahmens definiert das Land seine allgemeinen wirtschaftspolitischen Ziele sowie die konkreten Ziele für die Durchführung der GA. Im Rahmen einer ordnungspolitischen Regionalpolitik wäre das Land die erste Ebene unterhalb des Bundes, die konkrete regelgebundene Ziele und Programme definieren könnte. Daher sollen zunächst die wirtschaftspolitischen Ziele des Landes dargestellt werden und anschließend die regionalpolitischen.

\subsubsection{Allgemeine wirtschaftspolitische Ziele}

Es ist nicht ganz einfach, eine Quelle zu finden, die das wirtschaftspolitische Zielsystem des Landes beschreibt. In verschiedenen Publikationen des Wirtschaftsministeriums findet man teilweise explizit, teilweise aber auch nur implizit formulierte Ziele. 
Nimmt man hingegen eine Publikation der Staatskanzlei zur Hand ${ }^{123}$, findet man ein Zielsystem der Wirtschaftspolitik, dem zugleich die entsprechenden Instrumente und Mittel zugeordnet werden. Das übergeordnete wirtschaftspolitische Ziel ist wie folgt formuliert: "Für die Schaffung sicherer Arbeitsplätze durch eine wettbewerbsfähige Industrie, einen starken Mittelstand in Produktion, Handwerk und Dienstleistungen und mit moderner Infrastruktur"

Dieses allgemeine Ziel wird im folgenden weiter konkretisiert. Ich stelle die Bereiche dar, die einen regionalpolitischen Bezug haben.

\section{Verbesserung der regionalen Wirtschaftsstruktur}

Ziel: Sicherung und Schaffung von Arbeitsplätzen in überregional tätigen Unternehmen, Aufbau einer modernen wirtschaftsnahen Infrastruktur vorrangig in den Regionen mit überdurchschnittlich hoher Arbeitslosigkeit und Förderbedürftigkeit.

2. Förderung eines starken Mittelstandes in Produktion, Handwerk und Dienstleistungen

Ziel: Schaffung geeigneter Rahmenbedingungen für die Entwicklung eines gesunden und leistungsfähigen einheimischen Mittelstandes für zusätzliche Arbeits- und Ausbildungsplätze in der Wirtschaft

3. Förderung eines leistungsstarken und umweltverträglichen Tourismus

Ziel: Tourismuswirtschaft als wichtigen Wirtschaftsfaktor entwickeln; damit Wirtschaftswachstum induzieren und Arbeitsplätze schaffen. $\mathrm{M}-\mathrm{V}$ kontinuierlich als Ferienland innerhalb der nationalen Urlaubsgebiete ausbauen und an die nationale Spitze heranführen.

4. Förderung von neuen Technologien, Produktinnovationen und des Technologietransfers

Ziel: Steigerung des Anteils für Forschung und Entwicklung an den Unternehmensaufwendungen, Entwicklung neuer Technologien durch verstärkte Zusammenarbeit von Wirtschaft und Wissenschaft, Beschleunigung von Produktinnovationen und des Technologietransfers zur Steigerung der Wettbewerbsfähigkeit der Unternehmen und damit zur Schaffung und Sicherung von Arbeitsplätzen

5. Schaffung einer leistungsfähigen Verkehrsinfrastruktur

Ziel: Nachhaltige Verbesserung der Verkehrsträger Straße, Schiene, Seeund Flughäfen zur Erhöhung der Standortattraktivität und Nutzung der Chancen des Landes als Brückenkopf im Ostseeraum. Schaffung leistungsfähiger überregionaler Hauptverkehrsachsen. Verbesserung der Arbeitsund Lebensbedingungen in den Städten und im ländlichen Raum 
In diesem Zielsystem werden für die einzelnen Unterziele konkrete Maßnahmen gefordert. Basierend auf diesen Vorgaben könnten die notwendigen Instrumente und Maßnahmen geplant werden. Allerdings werden auch in diesem Zielsystem die Beziehungen der Ziele zueinander nicht berücksichtigt. Ferner werden keine eindeutigen Schwerpunkte gesetzt, so daß der Eindruck entsteht, mit den knappen finanziellen Mitteln könnten allen Ziele gleichzeitig erreicht werden.

Für die Wirtschaftsförderung wird eine Konzentration auf Gebiete mit hoher Arbeitslosigkeit gefordert. Primäres Ziel ist hier der Ausgleich innerhalb des Landes. Die Angleichung der Lebensverhältnisse in Mecklenburg-Vorpommern im Vergleich zum Bundesgebiet, die Förderung des Mittelstandes und die Technologieförderung verlangen jedoch eine eindeutige Ausrichtung am Wachstumsziel. Technologieförderung und Mittelstandsförderung muß sich auf leistungsfähige und dynamische Zentren konzentrieren, wenn ein spürbarer Wachstumsund Arbeitsplatzeffekt von der Förderung ausgehen soll. Und Angesichts der schlechten Infrastrukturausstattung muß auch diese zuerst in den dynamischen Zentren aufgebaut werden, damit die Mittelstands- und Technologieförderung nicht versickert. Denn, was nützen Technologieparks und moderne mittelständische Unternehmen, wenn der Engpaßfaktor Infrastruktur nicht beseitigt ist.

Der Ansatz eines konsistenten Zielbündels scheitert an den politischen Rationalitäten. Eine Landesregierung in Mecklenburg-Vorpommern muß die Förderung der Werften und des ländlichen Raumes als Priorität benennen, wenn sie ihre Macht sichern will. Auch wenn es ökonomisch nicht rational ist, Milliarden von Fördergeldern den Werften zur Verfügung zu stellen, scheint es aus Sicht der politischen Akteure notwendig.

\subsubsection{Ziele der Regionalförderung in Mecklenburg-Vorpommern}

Die Regionalförderung in Mecklenburg-Vorpommern steht vor einem Dilemma. Das Land ist gekennzeichnet durch dünne Besiedlung, wenige industrielle Zentren (deren Schwerpunkt zudem in den Problembereich Werftindustrie fällt), einen relativ hohen Anteil der Landwirtschaft am Sozialprodukt sowie die für einen Flächenstaat typischen Infrastrukturprobleme. Alle Prognosen gehen von einer rückläufigen Bevölkerungsentwicklung sowie zunehmenden Strukturproblemen für die Werftindustrie aus.

Auf der anderen Seite kann Mecklenburg-Vorpommern den Anschluß an die alten Bundesländer nur erreichen, wenn die Wirtschaft wächst und moderne, wettbewerbsfähige Arbeitsplätze geschaffen werden. Alle theoretischen Konzepte der Raumwirtschaft sowie empirische Untersuchungen zeigen sehr deutlich, daß Wachstum und Ansiedlung moderner und wettbewerbsfähiger Betriebe eine 
wesentliche Voraussetzung haben: Es müssen Zentren mit positiven Externalitäten existieren, in denen sich kommunikative Netzwerke bilden können. ${ }^{124}$

Die Regionalförderung in Mecklenburg-Vorpommern schwankt daher zwischen Ausgleichs- und Wachstumsziel. In der Anfangsphase ist keine klare Zielorientierung ablesbar. Im regionalen Förderprogramm für die Zeit 1992 - 1995 wird keine Aussage zu dieser Problematik gemacht. ${ }^{125}$ Die Ausführungen zur Förderpraxis lassen allerdings erkennen, daß in der ersten Phase das Ausgleichziel im Vordergrund stand. In den Regionalen Förderprogrammen für die Jahre 19931996 bzw. 1994-1997 stehen die beiden Ziele gleichrangig nebeneinander. ${ }^{126}$ Die Staatskanzlei des Landes betont hingegen die ausgleichspolitische Ausrichtung der Regionalförderung. Dies gilt sowohl für die Förderung von Gewerbebetrieben als auch für die Infrastrukturförderung.

Auch für den Bereich der Regionalförderung in Mecklenburg-Vorpommern gibt es kein konsistentes Zielsystem. Wie bei den wirtschaftspolitischen Zielen werden die vorhandenen Zielkonflikte weder benannt noch wird eine Lösung angeboten. Das Ausgleichs- und das Wachstumsziel lassen sich gleichrangig nebeneinander nicht erreichen. Die Landespolitik muß einem Ziel den Vorrang geben. Dies bedeutet jedoch nicht, daß das andere völlig vernachlässigt wird. ${ }^{127} \mathrm{Die}$ wachstumspolitisch notwendige Konzentration der Förderung auf die wirtschaftlichen Zentren des Landes hat erhebliche Ausstrahlungseffekte in den ländlichen Raum hinein. Über die Verteilung der Zentren kann die Streuung der positiven

Nach Erkenntnissen der Wachstumstheorie und empirischen Untersuchungen sind Zentren mit 100000 Einwohnern und einer Dichte von 150 Einwohnern je qkm von Relevanz. Vgl. R. Budde, R. Hamm, P. Klemmer u.a.: "Übertragung regionalpolitischer Konzepte auf Ostdeutschland", Untersuchung des RWI, Essen 1991.

Vgl. Einundzwanzigster Rahmenplan der Gemeinschaftsaufgabe "Verbesserung der regionalen Wirtschaftsstruktur", a.a.O., S.77 ff.

Vgl.: Zweiundzwanzigster Rahmenplan der Gemeinschaftsaufgabe "Verbesserung der regionalen Wirtschaftsstruktur", vom 19.05.1993, Drucksache 12/4850, S. 78/79 / Deutscher Bundestag: Dreiundzwanzigster Rahmenplan der Gemeinschaftsaufgabe "Verbesserung der regionalen Wirtschaftsstruktur", vom 28.04.1994, Drucksache $12 / 7175$.

In verschiedenen Studien wird ausdrucklich dafür plädiert, dem Wachstumsziel in den neuen Lăndern den Vorrang zu geben. Vgl. hierzu P. Klemmer et alia: "Übertragung regionalpolitischer Konzepte auf Ostdeutschland", a.a.O. / Prognos AG: "Instrumente und Informationssysteme fur die Wirtschaftspolitik des Landes Mecklenburg-Vorpommern", Untersuchung im Auftrag des Wirtschaftsministers des Landes MecklenburgVorpommern, 1992, S. 198-202. 
externen Effekte so gesteuert werden, daß sie in jeder Region des Landes wirksam werden. ${ }^{128}$

\subsection{Zusammenfassung: Das Zielsystem der Regionalpolitik für Mecklen- burg-Vorpommern}

Abbildung 1 stellt die oben diskutierten Ziele und ihre Beziehungen zueinander dar. Wichtigstes Ziel der Gemeinschaft ist die Kohäsion der Volkswirtschaften. Entsprechend dieser Vorgabe dient die Regionalpolitik dem Ausgleichsziel. Die fünf Unterziele, die regionale und funktionale Ziele miteinander vermengen, konkretisieren dieses Anliegen. Das Ergebnis der Konkretisierung sind Zielkonflikte. Aus einem in sich widersprüchlichen Zielsystem kann keine schlüssige Operationalisierung erfolgen. Diese Zielvorgaben sind bindend für die Mitgliedsstaaten. Sobald Mittel der Gemeinschaft zur Kofinanzierung nationaler Programme eingesetzt werden, müssen diese berücksichtigt werden.

Der Bund stellt auf der Verfassungsebene die Einheitlichkeit der Lebensverhältnisse in den Vordergrund. Die Bestimmungen des Grundgesetzes sehen vor, daß der Bund zur Erreichung dieses Zieles an der Wahrnehmung von Länderaufgaben beteiligt wird. Das Raumordnungsgesetz des Bundes konkretisiert den Verfassungsauftrag. Seine Zielvorgaben sehen sowohl die Förderung des ländlichen Raumes, als auch den Erhalt von Verdichtungsräumen vor. Die Raumordnung will eine geplante Raumstruktur, die einzelnen Gebieten bestimmte Funktionen und somit Entwicklungsmöglichkeiten zuweist, umsetzen. Dieser Ansatz einer zentralen Planung, der zudem die Länder und auch die Regionalpolitik bindet, kann nur begrenzt regionale Präferenzen berücksichtigen und Freiräume für einen Wettbewerb der Regionen schaffen. Die Regionalpolitik des Bundes orientiert sich an drei grundsätzlichen Zielen, zwischen denen es jedoch erhebliche Zielkonflikte gibt.

Das Land legt auf der Verfassungsebene den Schwerpunkt auf den Erhalt und die Schaffung von Arbeitsplätzen. Diese Zielvorgabe ist für alle Ebenen des Zielsystems bindend. Die wirtschaftspolitischen Ziele, das Landesraumordnungsgesetz und das Raumordnungsprogramm konkretisieren die Maßnahmen, mit denen das Ziel erreicht werden soll. Innerhalb dieses Zielsystems bleibt jedoch der Konflikt zwischen Ausgleichsziel und Wachstumsziel, der auch die Raumordnung und Regionalpolitik des Bundes bestimmt, ungelöst. Auf allen Ebenen gibt es Maßnahmen mit ausgleichspolitischer Zielsetzung und Maßnahmen mit wachstumspolitischer Zielsetzung.

128 Diese positiven Ausstrahlungseffekte von Zentren betont auch Giersch. Vgl. hierzu Herbert Giersch: "Kern und Rand in der spontanen Ordnung", FAZ vom 21.05.1994, S. 13. 
Abbildung 1: Regionalpolitische Ziele in Mecklenburg-Vorpommern

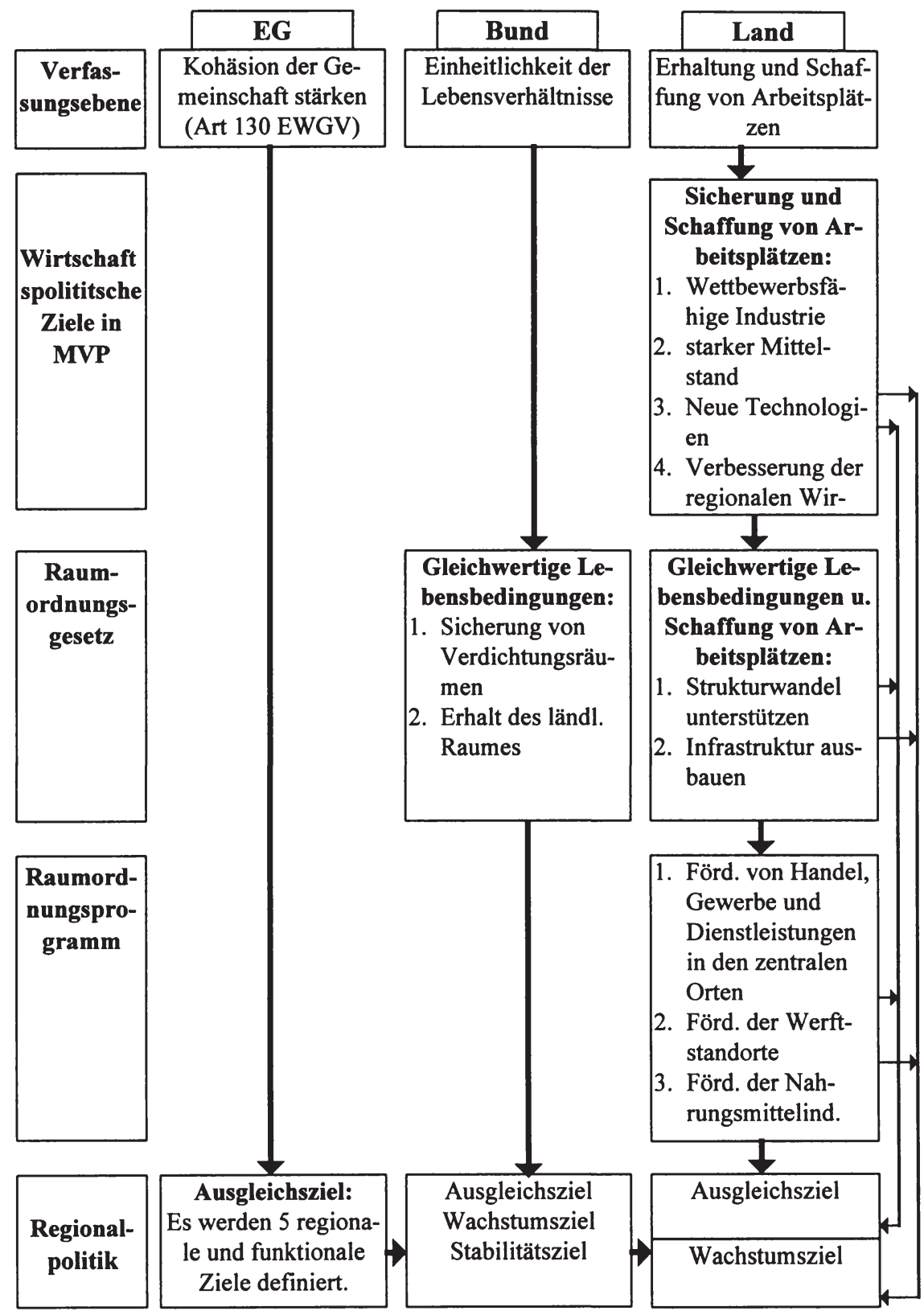


Die Handlungsfreiräume des Landes werden im Bereich der Raumordnung und der Regionalpolitik schon in der Zielsetzungsphase eingeschränkt. Allerdings lassen die Vorgaben des Bundes und der Gemeinschaft dem Land noch ausreichend Freiräume. Das Land hätte die Möglichkeit, die in den Zielen von Gemeinschaft und Bund angelegten Zielkonflikte zu lösen. Es könnte seine Politik z.B. eindeutig am Wachstumsziel orientieren.

Auf allen Ebenen zeigt sich eine Dominanz der politik-ökonomischen Rationalität, die eine Orientierung der Ziele an ökonomischen Rationalitäten verhindert. Es wird deutlich, daß verschiedenen Regionaltheorien bei der Formulierung und Fundierung der Ziele eine Rolle spielen. Die Defizite der Regionaltheorie, die ihre Umsetzung in politisches Handeln erschweren, spiegeln sich in den inkonsistenten Zielsystemen wieder. Der gewichtigste Grund für die unklaren und inkonsistenten Zielsysteme ist allerdings in politik-ökonomischen Gründen zu suchen. Ansätze für eine ordnungspolitische Regionalpolitik sind nicht zu sehen, es dominiert das konstruktivistische Konzept der Regionalpolitik, das die regionalen wirtschaftlichen Aktivitäten bewußt planen und steuern will.

\section{Regionalpolitik in Mecklenburg-Vorpommern: Die Instrumente Ge- meinschaftsaufgabe und EFRE}

Mit den Instrumenten der Regionalpolitik sollen die Ziele der verschiedenen Akteure umgesetzt werden. Da es kein konsistentes Zielsystem gibt, ist zu erwarten, daß auch die regionalpolitischen Programme nicht schlüssig sind. In wirtschaftspolitischen Programmen wird eine Mittel-Zweck-Beziehungen festgelegt, die auf einer der dargestellten Regionaltheorien beruht. Die Programme legen die Regeln für die Implementation fest und bieten eine Basis für die Evaluation. Hierbei spielen zunächst nur die Instrumente eine Rolle, die gemeinsam von der Gemeinschaft, dem Bund und den Ländern definiert, finanziert, umgesetzt und kontrolliert werden. Die Betrachtung beschränkt sich daher auf die Gemeinschaftsaufgabe und den EFRE.

In diesem Kapitel steht die institutionelle Struktur, in der die Programme definiert und implementiert werden, im Vordergrund. Die Analyse will folgende Fragen klären:

a. Wie sind in der jeweils relevanten institutionellen Struktur die Kompetenzen im Planungsprozeß zugeordnet? Welche Kriterien sind für die Entstehung der institutionellen Struktur ausschlaggebend?

b. Welches wirtschaftspolitische Konzept liegt den regionalpolitischen Programmen zu Grunde?

c. Welche Regionaltheorien werden zur Fundierung der Programme herangezogen? Gelingt es, basierend auf diesen Theorien, konsistente MittelZweck-Beziehungen zu formulieren? Wenn nein, warum nicht?

d. Gibt es Ansätze für eine ordnungspolitische Regionalpolitik? 
Der Gang der Untersuchung beginnt in diesem Kapitel mit der Landes- und Bundesebene. Die Gemeinschaftsaufgabe ist historisch gesehen die ältere Verflechtungsstruktur, die dann in die sich entwickelnde europäische Regionalpolitik integriert wurde. Ferner waren die Förderrichtlinien der GA im Untersuchungszeitraum auch für die EFRE Maßnahmen bindend. Anschließend wird dann die europäische Regionalpolitik mit Schwerpunkt auf dem EFRE untersucht.

\subsection{Die Gemeinschaftsaufgabe}

\subsubsection{Institutionelle Rahmenbedingungen}

Mit dem Einigungsvertrag erhielt das Gesetz über die „Gemeinschaftsaufgabe Verbesserung der regionalen Wirtschaftsstruktur" Gültigkeit für die fünf neuen Länder. ${ }^{129}$ Das Gesetz stammt aus dem Jahre 1969 und ist seitdem in seinen wesentlichen Bestandteilen unverändert. Wie bereits erwähnt, ist es in einer Phase von Bundestag und Bundesrat beschlossen worden, in der die Wirtschaftspolitik ökonomische Aktivitäten bewußt planen und steuern wollte. Ein wesentliches Ziel der GA war die Verhinderung bzw. Eindämmung eines Förderwettbewerbs zwischen den Ländern. Durch die Einführung eines gemeinsamen Planungs- und Steuerungsverfahren wurde dieses Ziel erreicht. Allerdings führte dies auch zu einer system-immanenten Tendenz der Anspruchsausweitung. ${ }^{130}$

Das Gesetz definiert in $\S 1$ Abs. 1 die Maßnahmen, die durch die GA im Bereich der gewerblichen Wirtschaft und der Infrastrukturförderung durchgeführt werden dürfen. ${ }^{131}$ Zentrales Steuerungsorgan der GA ist der Planungsausschuß, in dem der Bundeswirtschaftsminister und der Finanzminister für den Bund sowie die Länder Sitz und Stimme haben. Analog zu der finanziellen Lastenverteilung (Bund und Land tragen jeweils 50\%) sind auch die Stimmen gewichtet. Jedes Land hat eine Stimme, die Stimmenzahl des Bundes entspricht der Zahl der Länder. Beschlüsse kommen mit den Stimmen des Bundes und der Mehrheit der

Vgl. Einigungsvertrag vom 28.09.1990, Kap V, Sachgebiet A, Abschnitt III Nr. 1.

Die Fördergebiete haben sich kontinuierlich ausgeweitet - zuletzt lebten uber $40 \%$ der Bevolkerung in Fördergebieten. Erst durch den massiven Druck der Kommission im Rahmen ihrer Beihilfenkontrolle wurden die Fördergebiete eingeschränkt. Zuletzt wurde dies 1987 für das Jahr 1991 im sogenannten Bangemann-Sutherland-Kompromiß vereinbart. Der Anteil der Bevölkerung, der in Fördergebieten lebt, soll auf $27 \%$ verringert werden. Vgl. hierzu Deutscher Bundestag: Einundzwanzigster Rahmenplan der Gemeinschaftsaufgabe Verbesserung der regionalen Wirtschaftsstruktur, Drucksache 12/2599, vom 13.05.1992, S. 20.

Gesetz uber die Gemeinschaftsaufgabe Verbesserung der regionalen Wirtschaftsstruktur vom 06.10.1969 (BGBI. I, S. 1861). 
Länder zustande. ${ }^{132}$ In der derzeitigen Situation sind demnach neun Länder und die Stimmen des Bundes für eine Mehrheit erforderlich.

Der Planungsausschuß stellt den Rahmenplan auf, der Grundlage der GA ist und jedes Jahr sachlich zu prüfen, der Entwicklung anzupassen und dementsprechend fortzuführen ist. ${ }^{133}$ In ihm werden die Fördergebiete abgegrenzt, die regionalen Förderziele definiert sowie Voraussetzungen, Art und Intensität der Förderung bestimmt.

Der im Rahmen dieses Systems erstellte Rahmenplan muß von der Bundesregierung der Europäischen Kommission zur Genehmigung vorgelegt werden. Im Rahmen der Beihilfenkontrolle prüft die Kommission nationale Beihilfen auf ihre Vereinbarkeit mit dem gemeinsamen Markt. Gemäß Art 92 (1) EWGV sind "... staatliche oder aus staatlichen Mitteln gewährte Beihilfen gleichwelcher Art, die durch die Begünstigung bestimmter Unternehmen oder Produktionszweige den Wettbewerb verfälschen oder zu verfälschen drohen, mit dem Gemeinsamen Markt unvereinbar, soweit sie den Handel zwischen den Mitgliedstaaten beeinträchtigen."

Die Beihilfenkontrolle war zunächst auf die Förderung gewerblicher Investitionen beschränkt. Die Infrastrukturförderung, die im Rahmen der GA eine wichtige Rolle spielt, war nicht Gegenstand der Kontrolle. Helmut Neupert stellt noch 1986 hierzu fest: "Bei aller Weite des Beihilfenbegriffs des Art. 92 EWGV trägt Infrastrukturförderung im allgemeinen jedoch nicht den Charakter der Unternehmensbeihilfen und scheidet damit von vornherein aus der Beihilfenkontrolle aus." ${ }^{134}$ Seit der zweiten Hälfte der 80er Jahre dehnt die Kommission die Beihilfenkontrolle zunehmend auf Teilbereiche der wirtschaftsnahen Infrastruktur aus. ${ }^{135}$ Der Verkauf durch GA Mittel geförderter Gewerbegebiete an Unternehmen wird seitdem unter bestimmten Bedingungen als Subvention eingestuft. ${ }^{136}$ Im Zuge des Genehmigungsverfahrens für den 22. Rahmenplan hat die Kom-

Vgl. § 6 (1) und (2) Gesetz uber die Gemeinschaftsaufgabe Verbesserung der regionalen Wirtschaftsstruktur.

Vgl. § 4 (2) Gesetz über die Gemeinschaftsaufgabe Verbesserung der regionalen Wirtschaftsstruktur.

Helmut Neupert: "Regionale Strukturpolitik als Aufgabe der Länder: Grundlagen, Verknüpfungen, Grenzen", Baden-Baden 1986, S. 361.

Neben dem Verkauf von Gewerbeflächen an Unternehmen bezieht die Kommission die Überlassung von Räumen in Technologie-, Gründer- und Innovationszentren in die Beihilfenkontrolle ein.

Der Subventionscharakter hängt im wesentlichen vom Grundstückspreis ab. Liegt dieser unter dem Marktwert, ist in der Regel von einer Subvention auszugehen. Vgl. ausfuhrlich Arbeitskreis der EU-Wirtschaftsreferenten: "EG-Beihilfenkontrolle bezlglich Maßnahmen im Bereich der wirtschaftsnahen Infrastrukturen", Herbst 1994 / P. Schutterle: "EGBeihilfenkontrolle und kommunale Grundstücksverkăufe", in EuZW-Heft 20/1993. 
mission erstmals bestehende Beihilfesysteme unter diesem Aspekt überprüft und die Veränderung bestimmter Föderkonditionen gefordert. Dieser Forderung wurde dann im 23. Rahmenplan Rechnung getragen. ${ }^{137}$

\section{Mischfinanzierung und Politikverflechtung}

Die GA ist durch ein System der Mischfinanzierung und verflochtene Planungsund Steuerungsstrukturen gekennzeichnet. Die regionalpolitischen Entscheidungs- und Finanzierungskompetenzen werden zwischen Bund und Ländern aufgeteilt, eine klare Zuordnung wird mit dieser Strategie vermieden. Die theoretische Analyse hat gezeigt, daß dies eine Möglichkeit sein kann, externe Effekte öffentlicher Leistungen zu internalisieren. Die Mischfinanzierung bewirkt in diesem Fall jedoch das Gegenteil, da Kosten und Nutzen regionalpolitischer $\mathrm{Maßnahmen} \mathrm{auseinanderfallen.} \mathrm{Die} \mathrm{Förderung} \mathrm{wirtschaftsnaher} \mathrm{Infrastruktur}$ bzw. gewerblicher Investitionen nutzt, je nach Größe, Art und Ausstrahlungseffekt primär der Kommune, der Region bzw. dem Land. ${ }^{138}$ Die Kosten werden jedoch zu 50\% vom Bund mitgetragen, so daß das Land tendenziell an einer Ausweitung der Förderung interessiert sein muß, da der dem Land zukommende Nutzenanteil höher ist, als der Finanzierungsanteil. Die Länder sind somit daran interessiert, im Rahmen des Verhandlungsprozesses einen möglichst hohen Anteil der Bundesgelder zu erhalten. Im Rahmen dieses rent-seeking-Verhaltens orientieren sich Art und Umfang der Förderung nicht primär an regionalen Notwendigkeiten und Präferenzen, sondern an dem Ziel, möglichst viele Bundesmittel zu binden.

Die Berücksichtigung regionaler Präferenzen ist in diesem System nur in engen Grenzen möglich. Die neuen Länder mit ihren einmaligen Anpassungs- und Strukturproblemen, die die Deutsche Einheit und das Experiment der Transformation einer Plan- in eine Marktwirtschaft mit sich bringen, müssen sich eine Mehrheit für ihre Positionen suchen. Auch über die Landtage ist keine effektive Berücksichtigung der regionalen Präferenzen sichergestellt. Die im Rahmenplan festgelegten Mittel werden vom Bund bzw. von den Ländern in die Haushaltspläne aufgenommen. Die Landtage haben in dieser Phase keinen Einfluß mehr auf die inhaltliche Ausgestaltung des Rahmenplans. Sie könnten zwar den Haushaltsansatz streichen oder kürzen, dies hätte jedoch nur zur Folge, daß der Bun-

Vgl. Dreiundzwanzigster Rahmenplan der Gemeinschaftsaufgabe "Verbesserung der regionalen Wirtschaftsstruktur" vom 28.03.1994, Drucksache 12/7175, S.28 sowie Arbeitskreis der EU-Wirtschaftsreferenten: "EG-Beihilfenkontrolle bezüglich Maßnahmen im Bereich der wirtschaftsnahen Infrastrukturen", S. 2-4.

Diese Auffassung vertreten auch Gerhard Heimpold und Martin Junkernheinrich: "Der räumliche Streuungsbereich regionalpolitischer Maßnahmen liegt deutlich unterhalb der Landesebene, so daß sich eine Dezentralisierung der politischen Entscheidungskompetenzen anbietet." „Regionale Wirtschaftspolitik in den neuen Bundesländern“, in Institut für Wirtschaftsforschung Halle. "Wirtschaft im Systemschock", Berlin 1994, S. 166. 
desanteil im gleichen Umfang verändert würde. Auch gegenüber der Kommission haben die Länder nur eine schwache Verhandlungsposition. Für die im Rahmenplan festgelegten Programme ist der Bund Verhandlungspartner der Kommission (KOM). Direkte Verhandlungen zwischen Land und Kommission sind nur dann möglich, wenn das Land eigene Programme auflegt.

Im Rahmen der Gemeinschaftsaufgabe wird die Dominanz der politikökonomischen Rationalitäten noch verstärkt. Das verflochtene Planungs- und Steuerungssystem ist aufgrund seiner Struktur und Abstimmungsregel nicht in der Lage, Ansprüche einzelner Länder abzuwehren. Bei Einführung der Gemeinschaftsaufgabe wurden die bestehenden Fördergebiete, die vorher in bilateralen Absprachen zwischen dem Bund und dem jeweiligen Land festgelegt wurden, in das neue System übernommen. Eine Anpassungen der Förderung an neue regionale Probleme war in diesem System nur pareto-optimal möglich. Sollte z.B. eine Region neu aufgenommen werden, durfte dies nicht zu Lasten der vorhandenen Gebiete geschehen. Da jedoch jedes Land grundsätzlich an der Ausweitung seiner Fördergebiete interessiert war, fand sich stets eine Mehrheit für die pareto-optimale Ausweitung der Förderung.

Der Eigendynamik eines solchen Systems kann nur durch übergeordnete allgemeinverbindliche Regeln begegnet werden. Da auf der Bundesebene eine solche Politik nicht durchsetzbar war, müßte die Regelsetzung auf Gemeinschaftsebene erfolgen. Im Rahmen der Beihilfenkontrolle (Art 92 ff EWGV) werden staatliche Beihilfen grundsätzlich untersagt. Die Kommission hat das Recht, jede staatliche Beihilfe zu prüfen und notfalls zu versagen. Die Entwicklung der GA zeigt, daß der oben geschilderte Mechanismus erst durch die Auflagen der EG unterbrochen wurde, die die Bundesrepublik dazu zwangen, den Anteil der Fördergebiete an der Gesamtfläche auf $27 \%$ zu reduzieren. Durch die Ausweitung der Beihilfenkontrolle wurde der Einfluß der Kommission auf die GA noch weiter verstärkt. Da der Rahmenplan nur nach der Genehmigung durch die Kommission Gültigkeit erlangt, antizipieren Bund und Länder die Restriktionen der Kommission bereits im Aufstellungsverfahren des Rahmenplans. Trotzdem verzögert der Genehmigungsvorbehalt der Kommission oftmals das Inkrafttreten des Rahmenplans. Ursache hierfür sind Änderungswünsche der Kommission, die zumeist im Rahmen von Verhandlungen zwischen Bundesregierung und Kommission abgewiesen werden können.

Grundsätzlich scheint auf der Gemeinschaftsebene ein Ansatz für eine ordnungspolitische Regionalpolitik gegeben zu sein. Die Beihilfenkontrolle könnte Regeln für einen Wettbewerb der regionalen Einheiten setzen. Eine genauere Analyse in Kapitel acht wird untersuchen, ob die Realität der Beihilfenkontrolle diesem Anspruch gerecht wird. An dieser Stelle reicht zunächst der Hinweis, daß die Gemeinschaft neben der Beihilfenkontrolle eine eigene ziel- und programmorientierte Regionalpolitik betreibt. Es widerspricht dem Konzept der ord- 
nungspolitischen Regionalpolitik, wenn ein Akteur sowohl die Regeln setzt als auch selber konkrete Maßnahmen durchführt. Dies verleitet dazu, die allgemeinen Regeln zu Einzelfallregelungen zu mißbrauchen.

\subsubsection{Inhaltliche Vorgaben der GA}

Das Gesetz über die Gemeinschaftsaufgabe "Verbesserung der regionalen Wirtschaftsstruktur" sowie der Rahmenplan definieren die Fördertatbestände sowie die Förderarten, die im Rahmen der GA zulässig sind: ${ }^{139}$

1. Förderung der gewerblichen Wirtschaft bei Errichtung, Ausbau, Umstellung oder Rationalisierung von Gewerbebetrieben

2. Förderung beim Ausbau wirtschaftsnaher Infrastruktur: Erschließung von Gewerbegebieten, Ausbau von Verkehrsverbindungen, Energie- und Wasserversorgungsanlagen, Abwasser- und Abfallbeseitigungsanlagen, öffentliche Fremdenverkehrseinrichtungen, Errichtung bzw. Ausbau von Ausbildungs-, Fortbildungs- und Umschulungsstätten sowie Ausbau bzw. Errichtung von Gewerbezentren.

3. Förderungsarten: Investitionszuschüsse, Darlehen, Zinszuschüsse und Bürgschaften

Im Rahmenplan werden dann die Fördervoraussetzungen spezifiziert. Für die Infrastrukturförderung sowie für die Förderung der gewerblichen Wirtschaft gilt das Schwerpunktprinzip. Die Mittel sollen im Einklang mit der Landesplanung auf ausgewiesene Schwerpunktorte konzentriert werden. Hier finden sich die bereits erwähnten empirisch wie theoretisch belegten Erkenntnisse wieder, daß ein Mindestmaß an Agglomeration Voraussetzung für eine sich selbst tragende wirtschaftliche Entwicklung einer Region ist.

Investitionen der gewerblichen Wirtschaft müssen ferner dazu geeignet sein, "durch Schaffung von zusätzlichen Einkommensquellen das Gesamteinkommen in dem jeweiligen Wirtschaftsraum unmittelbar und auf Dauer nicht unwesentlich zu erhöhen (Primäreffekt). " ${ }^{140}$ Diese Voraussetzung kann als erfüllt angesehen werden, wenn der Absatz des Unternehmens überwiegend (mehr als 50\%) überregional erfolgt. Für die neuen Länder ist der Absatz bereits dann überregional, wenn er außerhalb eines Radius' von $30 \mathrm{~km}$ (alte Bundesländer $50 \mathrm{~km}$ )

Vgl. hierzu $§ 1$ (1) Nr. 1. und 2 Gesetz uber die Gemeinschaftsaufgabe "Verbesserung der regionalen Wirtschaftsstruktur", a.a.O.

Dreiundzwanzigster Rahmenplan, a.a.O., S. 22 (Hervorhebung durch den Verfasser). 
um die Gemeinde der Betriebsstätte liegt, oder der Betrieb in einem Bereich tätig ist, der in einer Positivliste vermerkt ist. ${ }^{141}$

Der Einigungsvertrag sieht für die neuen Länder die Möglichkeit der Abweichung von den im Gesetz bestimmten Regeln vor. Für folgende Bereiche können Abweichungen beschlossen werden: ${ }^{142}$

1. Bezüglich der Maßnahmen, die zur Förderung der gewerblichen Wirtschaft bzw. der Infrastruktur vorgesehen sind

2. Bezüglich der Förderungsarten

Diese Abweichungen werden im Rahmenplan festgelegt, d.h. der Einigungsvertrag öffnet die GA zwar grundsätzlich für neue Maßnahmen und Förderungsarten, überträgt jedoch dem Planungsausschuß die Entscheidungsbefugnis. Die neuen Länder waren erstmals an der Aufstellung des Zwanzigsten Rahmenplanes für den Zeitraum 1991-1994 beteiligt. In diesem Rahmenplan werden Ausnahmeregelungen für die fünf neuen Länder für einen Zeitraum von fünf Jahren beginnend am 03.10.1990 festgelegt. ${ }^{143}$ Diese sehen im wesentlichen eine Erhöhung der Fördersätze sowie eine Ausweitung der Fördertatbestände der GA vor. Im konzeptionellen Bereich wird auf das Schwerpunktprinzip verzichtet und das Exportbasiskonzept weiter verwässert. Im einzelnen wurden folgende Regelungen beschlossen:

a. Die neuen Länder müssen keine räumlichen Schwerpunkte für ihre Förderung schaffen (In den alten Bundesländern müssen Schwerpunkte gesetzt werden)

b. Es gelten höhere Fördersätze für Investitionszuschüsse: Errichtung 23\%, Erweiterung 20\%, Umstellung 15\%

c. Gewerbliche Fremdenverkehrsinvestitionen können mit bis zu 23\% gefördert werden (Übriges Bundesgebiet 15\%)

d. Hochwertige Arbeitsplätze mit einem Jahreseinkommen von mindestens 40.000 DM brutto (West: $60.000 \mathrm{DM}$ brutto) werden mit bis $\mathrm{zu} 25.000$ DM gefördert

141 Die Positivliste wird in jedem Rahmenplan veroffentlicht. Vgl. z.B. Dreiundzwanzigster Rahmenplan, a.a.O., S. 152.

Vgl. Einigungsvertrag vom 28.09.1990, Kap V, Sachgebiet A, Abschnitt III Nr. 1.

143

Vgl. Zwanzigster Rahmenplan der Gemeinschaftsaufgabe "Verbesserung der regionalen Wirtschaftsstruktur", vom 03.07.1991, Drucksache 12/895, S. 30. 
e. Die Förderhöchstsätze der regionalen Beihilfen können durch Beihilfen ohne regionalen Charakter (z.B. Investitionszulage) um bis zu 12\% erhöht werden $^{144}$

f. Als überregional gilt ein Absatz außerhalb eines Radius' von $30 \mathrm{~km}$ um die Gemeinde, in der die Betriebsstätte liegt (West: $50 \mathrm{~km}$ )

g. Möglichkeit der Förderung des Bauhaupt- und Ausbaugewerbes bis zum 31.12.1992 (In den alten Bundesländern nicht zulässig)

Zusätzlich zu diesen Maßnahmen wurden im Zuge der jährlichen Anpassungen der Rahmenpläne weitere Ausnahmen aufgenommen:

h. In Regionen, die besonders von Stillegungen betroffen sind, werden Hilfen für Beratungsmaßnahmen zur Ansiedlungsförderung und Projektdurchführung gewährt. (In den alten Ländern nicht zulässig) ${ }^{145}$

i. Wenn in Regionen, die in besonderem Maße von Arbeitslosigkeit betroffen sind, bei Ausnutzung aller bestehenden Beihilfen die Förderhöchstsätze nicht erreicht werden, können die Investitionszuschüsse in Ausnahmefällen so erhöht werden, daß die Förderhöchstsätze erreicht werden (in den alten Bundesländern nicht zulässig) ${ }^{146}$

j. Vor der Bewilligung von Infrastrukturfördermitteln, soll der Träger der Maßnahme im Rahmen eines Interessenbekundungsverfahren prüfen, ob durch Einschaltung privater Unternehmen Kosten- bzw.- Zeitvorteile erzielt werden können (in den alten Bundesländern nicht vorgesehen) ${ }^{147}$

\section{Bewertung}

Die GA basiert auf einem konstruktivistischen wirtschaftspolitischen Konzept. Sie orientiert sich an einem regional- und strukturpolitischem Leitbild, das mit den Maßnahmen der GA implementiert werden soll. Die GA bezieht sich explizit auf die Exportbasistheorie und die Theorie der Wachstumspole. Jedoch lassen sich auch Elemente aus den neuen Wachstumsmodellen erkennen.

Auf die theoretischen und praktischen Probleme der Exportbasistheorie wurde bereits hingewiesen. Am Beispiel der Regionalförderung in Mecklenburg-

\footnotetext{
144

Die Errichtung eines Gewerbebetriebes kann bei einem Fördersatz von $23 \%$ durch weitere nicht regional spezifizierte Beihilfen auf einen Fördersatz von $35 \%$ erhöht werden.

Vgl. Einundzwanzigster Rahmenplan der Gemeinschaftsaufgabe "Verbesserung der regionalen Wirtschaftsstruktur" vom 13.05.1992, Drucksache 12/2599, S. 29.

Vgl. Zweiundzwanzigster Rahmenplan der Gemeinschaftsaufgabe "Verbesserung der regionalen Wirtschaftsstruktur" vom 19.05.1993, Drucksache 12/4850, S. 26/27. regionalen Wirtschaftsstruktur" vom 28.03.1994, Drucksache 12/7175, S.29.
} 
Vorpommern läßt sich die Unzulänglichkeit der Theorie und ihrer Umsetzung darstellen. Da die Theorie keinen Hinweis gibt, welche Industrien exportorientiert sind, hilft sich die Regionalförderung mit einer räumlichen Abgrenzung. Betrachtet man z.B. die Stadt Schwerin als einen möglichen Standort für einen Gewerbebetrieb, dann wäre das Kriterium des überregionalen Absatzes bereits erfüllt, wenn die Produkte in den umliegenden Kreisen vertrieben würden. Das zusätzliche Einkommen aus dem überregionalen Absatz wird somit in Kreisen erwirtschaftet, deren wirtschaftliche Situation weitaus schlechter ist als an dem geförderten Gewerbestandort Schwerin. Schwerin selber ist aus Sicht der umliegenden Kreise ein Gebiet, in dem überregionaler Absatz getätigt werden kann. $\mathrm{Da}$ die neuen Länder in ihrer Gesamtheit Förderregion sind, ist jeder Kreis potentielle Quelle für zusätzliche Einkommen aus überregionalem Absatz.

Die Förderbedingungen, mit denen dieses Konzept umgesetzt werden sollen, führen den Gedanken der Exportbasistheorie ad absurdum. Es wäre daher ehrlicher, die Bedingung des überregionalen Absatzes zu streichen. Das Land Mecklenburg-Vorpommern hat zuletzt im Sommer 1994 versucht, den Primäreffekt als Förderkriterium zu streichen. ${ }^{148}$ Die Wirtschaft in Mecklenburg-Vorpommern ist durch kleine und mittlere Unternehmen sowie Handwerksbetriebe geprägt. Nach Ansicht des Landes erfüllen nur wenige dieser Unternehmen das Kriterium des überregionalen Absatzes, so daß eine gezielte Förderungen von kleinen und mittleren Unternehmen als Keimzelle bzw. Basis für einen weiteren Aufbau exportorientierter Unternehmen mit dem Instrument der GA nur eingeschränkt möglich ist. Die Position des Landes fand jedoch sowohl bei den Ländern als auch beim Bund keine Unterstützung. ${ }^{149}$

Auch die Umsetzung der Theorie der Wachstumspole und der neuen Wachstumsmodelle zeigt die erwartbaren Schwierigkeiten. Die GA schafft insbesondere durch die Sonderregelungen ein Fördergefälle zugunsten der fünf neuen Länder. Die Höchstsätze der Förderung mit regionalem Charakter liegen deutlich über den Fördersätzen in den alten Bundesländern. In Verbindung mit nicht regional spezifizierten Subventionen (z.B. Investitionszulage und Sonderabschreibungen) kann eine Investition in den neuen Ländern auch dann noch interessant sein, wenn sie keinen positiven Grenzertrag abwirft. ${ }^{150}$

Obwohl dynamische Wirtschaftszentren Bedingung für Wachstumspole und die Erzeugung externer Effekte sind, werden die neuen Länder von der Verpflichtung befreit, räumliche Schwerpunkte für die Förderung zu definieren. Aufgrund der enormen regionalpolitischen Probleme sowie in Ermangelung der notwendi-

Vgl. interner Vermerk des Wirtschaftsministeriums vom 21.07.1994.

Vgl. interner Vermerk des Wirtschaftsministeriums vom 14.09.1994.

Vgl. ifo studien zur finanzpolitik 53: "Die Effizienz der finanzpolitischen Fördermaßnahmen in den neuen Bundeslăndern", Münschen 1994, S. 34/35.
} 
gen Daten setzte der Planungsausschuß keine Förderschwerpunkte für die neuen Länder fest. Dies geschah aber auch mit der Absicht, den Ländern die Möglichkeit zu geben, "... bei der Förderung in Kenntnis der regionalen Entwicklungspotentiale und -hemmnisse räumliche Schwerpunkte selbst zu setzen." ${ }^{151}$

Trotz der erheblichen Mittel, die die Europäische Gemeinschaft, der Bund und die Länder zur Verfügung gestellt haben - für Mecklenburg-Vorpommern standen pro Jahr ca. 1 Mrd. DM zur Verfügung - ist angesichts des enormen Anpassungs- und Modernisierungsbedarfs das Setzen von Prioritäten erforderlich. ${ }^{152}$ Obwohl diese Notwendigkeit erkannt wird, entbindet man die neuen Länder von der Pflicht, räumliche Schwerpunkte zu definieren, da es nicht möglich erscheint, diese "... im Umstrukturierungsprozeß in den neuen Ländern zu erkennen ..." sowie "... Investitionen außerhalb von möglichen Schwerpunktorten gegenüber denjenigen in Schwerpunktorten zu benachteiligen." ${ }^{153}$ Die ohnehin dünne theoretische Basis hat faktisch keine Bedeutung mehr, Regionalförderung findet mehr oder weniger zentral gesteuert auf Verdacht statt. Dies bedeutet in der Konsequenz ein Scheitern des wirtschaftspolitischen Ansatzes, da der Anspruch, wirtschaftliche Entwicklungen planen und steuern zu können, aufgegeben wird.

Der Verzicht auf Schwerpunktsetzung führt zu Unstimmigkeiten und Zielkonflikten mit den anderen regionalpolitisch relevanten Bereichen. Der Rahmenplan verlangt als allgemeine Zulässigkeitsvoraussetzung von gewerblichen Investitionen bzw. Infrastrukturmaßnahmen eine Übereinstimmung mit der Landesplanung. Wie oben dargestellt, ist es originäre Aufgabe der Landesplanung, dem Raum bestimmte Funktionen zuzuweisen und die wirtschaftliche Entwicklung in Schwerpunktorten zu fördern. Wenn die Verbindung zwischen GA und Landesplanung eingehalten werden soll, muß sich die Förderung an Schwerpunkten orientieren.

Die inhaltlichen Vorgaben der GA basieren somit auf einem wirtschaftspolitischen Konzept, das nicht durchführbar ist. Entweder ist die Umsetzung der Theorie in praktische Politik unsinnig (Exportbasistheorie) oder die theoretisch begründeten Förderschwerpunkte werden Mangels ausreichender Informationen

Michael Zarth: "Neuordnung der Regionalforderung im Rahmen der Gemeinschaftsaufgabe Verbesserung der regionalen Wirtschaftsstruktur", in Informationen zur Raumentwicklung, Heft 9/10.1991, S. 554.

Diese Auffassung wird auch im Bundeswirtschaftsministerium geteilt. Iris HenselerUnger sagt: "Die neuen Länder sind gezwungen, bei der Forderung durch Investitionszuschüsse angesichts der knappen Mittel eigene Priorităten zu setzen.": "Regionale Wirtschaftsforderung in den neuen Bundesländern", in Jahrbuch fur Regionalwissenschaft, 12./13. Jahrgang, S. 63.

Iris Henseler-Unger: "Regionale Wirtschaftsforderung in den neuen Bundesländern", a.a.O., S. 63 . 
Bindung an die Vorgaben der GA. Diese Beliebigkeit der inhaltlichen Regelungen im Bereich der Wirtschaftsförderung gibt den Ländern zwar gewisse Handlungsspielräume, führt jedoch auch dazu, daß keine klaren allgemeinverbindlichen Regeln für die Regionalpolitik existieren.

\subsection{Einsatz des EFRE in Mecklenburg-Vorpommern}

Wie oben bereits dargestellt, steht der Ausgleich zwischen armen und reichen Regionen in der Gemeinschaft im Zentrum der europäischen Regionalpolitik. Die neuen Länder bilden mit Portugal, Spanien, Griechenland und Irland das untere Ende der Wohlstandsskala der Gemeinschaft. Das Pro-Kopf-BIP in den fünf neuen Ländern erreichte nach Schätzungen der Kommission nur 35\% des Gemeinschaftsdurchschnitts. ${ }^{154}$ Bereits im Dezember 1990 beschloß der Rat, die neuen Länder in die Strukturpolitik der Gemeinschaft zu integrieren. ${ }^{155}$ Die Gemeinschaft stellte drei Mrd. Ecu für die Jahre 1991 bis 1993 zur Verfügung und verzichtete mangels statistischer Daten auf eine "... vorherige Einstufung der Regionen und Gebiete dieses Gebietes nach den regionalpolitischen Zielen...". ${ }^{156}$ Im folgenden sollen die institutionellen Rahmenbedingungen der europäischen Strukturpolitik für die Zeit von 1991 bis 1993 sowie die inhaltlichen Vorgaben diskutiert werden.

Von 1994 an gilt eine neue Strukturfondsverordnung, da die bestehende Verordnung 1993 überprüft und angepaßt wurde. Für die Zeit von 1994 bis 1999 entfallen die Ausnahmeregelungen für die neuen Länder. Die Grundprinzipien der Strukturpolitik bleiben bestehen, allerdings kommt es durch verschiedene Veränderungen zu einer weiteren Bürokratisierung. Eine vertiefte Diskussion der neuen Fondsverordnung erfolgt in Kapitel neun.

\subsubsection{Institutionelle Rahmenbedingungen der EG Strukturpolitik}

Die wichtigsten Prinzipien der Reform der Strukturfonds von 1988 waren Konzentration, Programmplanung, Partnerschaft und Zusätzlichkeit. Im Rahmen der Partnerschaft zwischen Kommission und nationalen bzw. regionalen Behörden werden Programme entwickelt, die die Vergabe der zusätzlich zu den nationalen bzw. regionalen Mitteln zur Verfügung gestellten Strukturfondsgelder regeln.

Vgl. Europäische Kommission: "Wettbewerbsfăhigkeit und Kohäsion: Tendenzen in den Regionen - Fünfter periodischer Bericht uber die soziő̈konomische Lage und Entwicklung der Regionen der Gemeinschaft", Luxemburg 1994, S. 40.

Vgl. Verordnung (EWG) Nr. 3575/90 des Rates vom 4. Dezember 1990 ubber die Intervention der Strukturfonds im Gebiet der ehemaligen Deutschen Demokratischen Republik.

Artikel 2 (5) Verordnung (EWG) Nr. 3575/90 des Rates. 
Die Bundesregierung wurde verpflichtet, bis zum 31.01.91 für die neuen Länder einen Regionalentwicklungsplan für die Strukturinterventionen vorzulegen. Dieser Plan mußte eine "... möglichst detaillierte Analyse der sozio-ökonomischen Lage ..." ${ }^{157}$ der neuen Länder, eine auf angemessener regionaler Ebene erstellte Beschreibung der Gemeinschaftsinterventionen und ihre Schwerpunkte sowie Angaben zur Verwendung der Fonds enthalten.

Die Kommission prüft, ob die vorgeschlagenen Pläne mit den Zielen der Strukturfondsverordnung übereinstimmen und legt daraufhin "... im Rahmen der Partnerschaft ... und im Einvernehmen mit dem betreffenden Mitgliedstaat ... das gemeinschaftliche Förderkonzept ..." für die Strukturinterventionen der Gemeinschaft fest. ${ }^{158}$ Partnerschaft bedeutet eine enge Konzertierung zwischen der Kommission, dem Mitgliedstaat und den betroffenen nationalen, regionalen und lokalen Behörden. Alle Parteien sollen als Partner "... ein gemeinsames Ziel verfolgen." 159

Um eine möglichst reibungslose Antragsbearbeitung für konkrete Interventionen zu gewährleisten, legte das Land Mecklenburg Vorpommern für die Mittel des EFRE ein sogenanntes operationelles Programm vor. ${ }^{160}$ Ein operationelles Programm ist "... ein kohärentes Bündel mehrjähriger Maßnahmen, zu deren Durchführung ein oder mehrere Fonds ..." ${ }^{161}$ eingesetzt werden können. Derartige Programme sind nicht zwingend vorgeschrieben, jedoch werden sie von der Kommission für Interventionen im Rahmen der Ziele 1 und 2 erwartet. ${ }^{162}$

Das Gemeinschaftliche Förderkonzept (GFK) sieht einen begleitenden Ausschuß für das Gebiet der fünf neuen Länder vor. Diesem Ausschuß stehen sechs Unterausschüsse zur Seite, die jeweils für ein Land zuständig sind. In dem Ausschuß ist die Kommission, die Europäische Investitionsbank (EIB) sowie der Mitgliedstaat und die betroffenen nationalen und regionalen Behörden vertreten. Für

Artikel 2 (1) Verordnung (EWG) Nr. 3575/90 des Rates, Hervorhebung durch den Autor.

Artikel 8 (5) Verordnung (EWG) Nr. 2052/88 des Rates, Abl. Nr. L 158/9 vom 15.07.1988.

Artikel 4 (1) Verordnung (EWG) Nr. 2052/88 des Rates, Abl. Nr. L 158/9 vom 15.07.1988.

Ministerium fur Wirtschaft des Landes Mecklenburg-Vorpommern: "Operationelles Programm des Europäischen Fonds fur regionale Entwicklung fur das Land MecklenburgVorpommern im Zeitraum 1991-1993" vom 08.03.1991.

Artikel 5 (5) Verordnung (EWG) Nr. 2052/88 des Rates, Abl. Nr. L 158/9 vom 15.07.1988.

Vgl. Artikel 8 (6) und Artikel 9 (10) Verordnung (EWG) Nr. 2052/88 des Rates, Abl. Nr. L 158/9 vom 15.07.1988. 
die Unterausschüsse wird die Beteiligung oder Konsultierung der Sozialpartner vorgeschlagen. ${ }^{163}$

Der begleitende Ausschuß soll den Mitteleinsatz koordinieren, beurteilen und Änderungsvorschläge prüfen. Ferner ist er für die Erhebung von Indikatoren verantwortlich, die eine Bewertung der Maßnahmen zulassen. Das Gemeinschaftliche Förderkonzept sieht eine umfassende Aufschlüsselung verschiedener Indikatoren vor, deren Erhebung und Bearbeitung einen erheblichen Aufwand darstellt. Neben dieser laufenden Kontrolle verlangt das GFK einen jährlichen Lagebericht sowie einen Schlußbericht nach Abschluß der Aktion. ${ }^{164}$

\section{Politikverflechtung und Mischfinanzierung}

Ziel der Gemeinschaft ist eine eigenständige europäische Regional- und Strukturpolitik. Sie versucht deshalb, im Rahmen der bestehenden Verflechtungsstrukturen ihren Einfluß auf die regionalpolitischen Programme des Bundes und der Länder auszuweiten. Die europäische Regionalpolitik ist noch mehr als die bundesdeutsche durch die negativen Folgen der Mischfinanzierung und der Politikverflechtung gekennzeichnet. Neben Bund und Länder tritt eine dritte Ebene, die eigene regionalpolitische Ziele mit eigenen Instrumenten verfolgt. Eine Koordinierung der regionalpolitischen Ziele und Programme mit der Kommission wird durch folgende Faktoren erschwert:

1. Die Ziele der GA und der europäischen Strukturpolitik sind nur sehr eingeschränkt kompatibel. Die GA konzentriert sich auf die Ziele Wachstum, Stabilität und Ausgleich, wobei den Ländern vorbehalten ist, den Schwerpunkt frei zu wählen. Die Kommission gibt basierend auf dem Ausgleichsziel ein inkonsistentes Zielbündel vor. Beschränken sich Bund und Länder auf die mit der GA kompatiblen Ziele, riskieren sie stets den Widerspruch der Kommission.

2. Die Politik der Kommission basiert auf einem aus der zentralistischen französischen Tradition erwachsenen Staatsverständnis. Dieses kollidiert zwangsläufig mit dem förderalistischen Staatsverständnis der Bundesrepublik.

3. Die Kommission hat keinen eigenen Verwaltungsunterbau. Sie ist bei der Implementation ihrer Programme auf die Mitarbeit der nationalen bzw. regionalen Behörden angewiesen.

\footnotetext{
163 Vgl. Kommission der Europăischen Gemeinschaften: "Gemeinschaftliches Förderkonzept für die Gebiete von Ost-Berlin, Mecklenburg-Vorpommern, Brandenburg, SachsenAnhalt, Thüringen und Sachsen 1991-1993", Brussel, 13.03.1991, S. 43/44.

Vgl. "Gemeinschaftliches Forderkonzept 1991-1993", a.a.O., S. 45.
} 
Im Ergebnis führt dies zu einer immer weiter fortschreitenden Bürokratisierung der Regionalpolitik. Die Kommission möchte in den Mitgliedstaaten und in den Regionen eigene Ziele und Programme durchsetzen sowie die Verwendung ihrer Gelder kontrollieren. Engpaß dieses ehrgeizigen Vorhabens ist die Informationsbeschaffung und Verarbeitung durch die Kommission. Es ist eine „Anmaßung von Wissen "165, wenn die Kommission versucht, für jedes Mitglied, für jede Region maßgeschneiderte Entwicklungsprogramme zu entwerfen. Man kann diese Entwicklung an einem einfachen Wirkungsschema verdeutlichen.

Abbildung 2: Wirkungsschema der Politikverflechtung

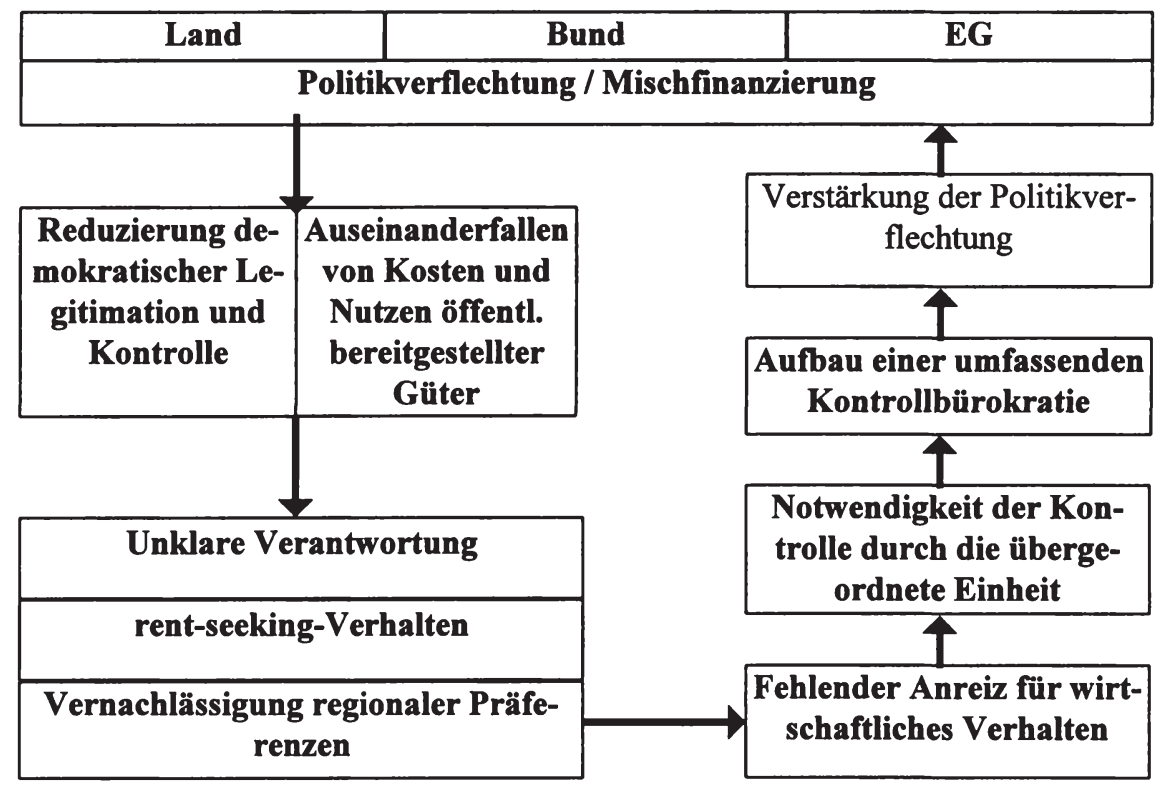

Durch Mischfinanzierung und Politikverflechtung fallen Kosten und Nutzen der regionalpolitischen Programme auseinander. Dies führt zu einem massiven rentseeking-Verhalten der unteren Ebenen und zu einer Verwischung der Verantwortung für die durchgeführten Projekte. Ferner ist die demokratische Kontrolle fast ausgeschaltet. Diese Strukturen bieten keinen Anreiz für einen wirtschaftlichen Umgang mit den vorhandenen Geldern, außerdem kommt es zu allokativer Ineffizienz, da die regionalen Präferenzen vernachlässigt werden. Die logische Re- 
aktion des Systems ist eine massive Ausweitung der Kontrolle, eine Verdichtung der Reglementierungen und somit eine Verstärkung der Verflechtung. Dies endet dann im Ergebnis in einem total verbürokratisierten System der Regionalpolitik, quasi der Schaffung einer "zweiten EG-Agrarpolitik". ${ }^{166}$

Dieses System hat keine Ansätze für eine ordnungspolitische Regionalpolitik. Über die Beihilfenkontrolle hätte die Kommission die Möglichkeit, allgemeingültige Regeln für einen Wettbewerb der regionalen Einheiten zu definieren. Da sie als regionalpolitischer Akteur jedoch eigene Ziele und Interessen verfolgt, kann sie die Funktion der Regelsetzung und Durchsetzung nicht mehr glaubwürdig wahrnehmen. Jede beteiligte Ebene hat ein Interesse, einen Teil der Kompetenzen für sich zu beanspruchen. Die politik-ökonomischen Rationalitäten stabilisieren und verfestigen die Verflechtungsstrukturen.

\subsubsection{Inhaltlichen Vorgaben der EG Strukturpolitik}

Die Betrachtung der unterschiedlichen Vorgaben beschränkt sich auf die EG Mittel, die über den Haushalt des Schweriner Wirtschaftsministeriums verwaltet werden, d.h. auf die Mittel des EFRE. Die Mittel des EFRE tragen in Mecklenburg-Vorpommern "... zur Verstärkung der nationalen Mittel im Rahmen der Gemeinschaftsaufgabe Verbesserung der regionalen Wirtschaftsstruktur bei." ${ }^{167}$ Die Finanzierung erfolgt somit aus Mitteln des EFRE sowie aus Mitteln der GA des Landes und des Bundes. Sowohl in den Finanzplänen der Rahmenpläne als auch im Haushaltsplan des schweriner Wirtschaftsministeriums ist die notwendige Kofinanzierung der EFRE Mittel nicht eindeutig ersichtlich. In dem für diese Untersuchung relevanten Zeitraum wurde die Verbuchungspraxis laufend geändert. Die Übersichtlichkeit und Vergleichbarkeit der Zahlen wird dadurch erheblich erschwert. ${ }^{168}$

\footnotetext{
166 Rudolf Ridinger: "Aktuelle Diskussionen zur Finanzausstattung und Reform der EGRegionalforderung", a.a.O., S. 654.

Ministerium für Wirtschaft Mecklenburg-Vorpommern: "Operationelles Programm 19911993", a.a.O., S. 24.

168 Im zwanzigsten und einundzwanzigsten Rahmenplan werden die EG Mittel uberhaupt nicht erwähnt. Erst im zweiundzwanzigsten Rahmenplan für 1993-1996 werden die EFRE Mittel für die fünf neuen Lănder beziffert. Im dreiundzwanzigsten Rahmenplan werden die EFRE Mittel sowie die Kofinanzierung durch den Bund zum erstenmal explizit ausgeworfen.
} 
Das Gemeinschaftliche Förderkonzept für die neuen Länder sieht eine erheblich breitere Palette von förderungsfähigen Maßnahmen vor als die GA. ${ }^{169}$ Basierend auf den Fondsverordnungen werden aus vier globalen Entwicklungszielen acht Förderschwerpunkte abgeleitet. ${ }^{170}$ Das operationelle Programm für den Einsatz der EFRE Mittel in Mecklenburg-Vorpommern konzentriert sich auf vier der acht Schwerpunkte. Die im Rahmen dieser Schwerpunkte förderungsfähigen Maßnahmen entsprechen im wesentlichen den Regelungen der GA. ${ }^{171}$ Für die Jahre 1991 bis 1993 ist eine enge Anlehnung der europäischen Förderung an die Gemeinschaftsaufgabe allein schon durch die Größenordnung der bereitgestellten Mittel geboten. Der Anteil der EFRE Mittel an den regionalpolitischen Fördermaßnahmen in Mecklenburg-Vorpommern lag zwischen 19\% 1991 und 11\% 1993. ${ }^{172}$ Dies wird auch von der Kommission so gesehen, die im Gemeinschaftlichen Förderkonzept feststellt: "Die Intervention der EG-Strukturfonds ist zwar aus Sicht der Gemeinschaft bedeutend, doch nur Teil eines viel umfassenderen Programms, das von der Bundesrepublik finanziert wird. Dabei ist der Einordnung des Gemeinschaftsanteils in diesen breiteren Rahmen Priorität beizumessen. ${ }^{173} \mathrm{Im}$ Ergebnis bedeutet dies, daß für den Zeitraum 1991-93 die inhaltlichen Vorgaben der Gemeinschaftsaufgabe maßgeblich waren. Erst ab 1994, mit dem Auslaufen der Ausnahmeregelungen, dem Inkrafttreten einer neuen Fondsverordnung sowie einer erheblichen Aufstockung der EFRE Mittel für die neuen Länder, gewann die Kommission einen größeren Einfluß auf die Ausgestaltung der inhaltlichen Vorgaben. ${ }^{174}$

Für die Regionalpolitik im Rahmen des EFRE gilt somit zunächst alles das, was zur theoretischen Fundierung der Gemeinschaftsaufgabe gesagt worden ist. Die Kommission hält jedoch an dem Anspruch der Plan- und Steuerbarkeit regiona-

Auch für die Gelder des ESF, die úber die BA vergeben wurden, gilt dieser Befund. Mit diesen Mitteln wurden u.a. Projekte finanziert, die durch das recht umfassende Programmangebot des AFG nicht abgedeckt waren. Vgl. Kommission der Europäischen Gemeinschaften: "Gemeinschaftliches Förderkonzept für die Entwicklung und die strukturelle Anpassung der Regionen mit Entwicklungsruckstand (Ziel 1) 1994-1999" a.a.O., S. 24.

Vgl. Kommission der Europăischen Gemeinschaften: "Gemeinschaftliches Förderkonzept 1994-1999", a.a.O., S 17ff.

Vgl. Ministerium fur Wirtschaft Mecklenburg-Vorpommern: "Operationelles Programm 1991-1993", a.a.O., S. 6.

Basis dieser Berechnung sind die in dem Hauhalt des schweriner Wirtschaftsministeriums veranschlagten Ausgaben der Gemeinschaftsaufgabe, des Gemeinschaftswerkes Aufschwung Ost sowie des EFRE.

Kommission der Europäischen Gemeinschaften: "Gemeinschaftliches Förderkonzept 1994-1999", a.a.O., S. 28.

Siehe hierzu Abschnitt 9. 
ler wirtschaftlicher Entwicklungen fest. Sie fordert für die Programme, die sie mitfinanziert, schlüssige wirtschaftspolitische Programme, die klare Ziel-MittelBeziehungen darstellen und überprüfbar sind. Neben einer Analyse der Ausgangslage werden Entwicklungsziele definiert, aus denen die Förderschwerpunkte abgeleitet werden. Diese bilden die Grundlage für die konkreten förderfähigen Maßnahmen. Die Länder müssen diese Programme über den Bund der Kommission zur Genehmigung vorlegen. Im Rahmen einer ordnungspolitischen Regionalpolitik wäre auf Länderebene eine detaillierte und überprüfbare Programmplanung durchaus wünschenswert. Wie die Analyse der Verflechtungstrukturen jedoch gezeigt hat, werden diese Maßnahmenprogramme nicht im Rahmen einer regelgebundenen Wettbewerbsordnung aufgestellt, sondern ganz maßgeblich inhaltlich durch die zentrale Instanz EG mitbestimmt. Hat der Bund im Rahmen der Gemeinschaftsaufgabe den Anspruch auf Steuerung der regionalen Entwicklung teilweise aufgegeben, so besteht die Gemeinschaft nachwievor auf diesem Anspruch und setzt ihn mittels einer Kontrollorganisation auch durch.

Die größere Breite der förderfähigen Maßnahmen - insbesondere im Bereich der Infrastruktur sowie der gewerblichen Wirtschaft - könnte durchaus positiv bewertet werden. Die Länder können im Rahmen ihrer operationellen Programme diese Fördermaßnahmen nach ihren eigenen Präferenzen kombinieren und eigene Schwerpunkte setzen. Damit wäre grundsätzlich ein höherer Grad der allokativen Effizienz erreichbar.

Allerdings gibt es andere Faktoren, die diesen Effekt aufheben können. Zum einen können die regionalen Präferenzen lediglich im Rahmen der Partnerschaft durchgesetzt werden. Das Land muß Bund und Kommission von seinem Programm überzeugen, damit es letztendlich von der Kommission genehmigt wird. Im Ergebnis können sich die Präferenzen des Bundes bzw. der Kommission durchsetzen.

Ferner muß kritisch hinterfragt werden, wer auf der Landesebene die Präferenzen artikuliert, die dann Basis für ein Förderprogramm werden. Wie oben dargestellt, findet der Verhandlungsprozeß im wesentlichen zwischen den Landes- und Bundesministerien statt. Die regionalen Behörden werden in der Regel nicht beteiligt. In der derzeitigen Situation sind die Präferenzen des Landes identisch mit den Präferenzen der Bürokratie.

Dies führt dazu, daß jedes Haus im Rahmen einer Budgetmaximierungsstrategie an möglichst hohen Zuweisungen von Bund und EG interessiert ist. Dadurch, daß der Landesanteil bei EG kofinanzierten Projekten nur noch $25 \%$ beträgt, ist die Motivation zum rent-seeking besonders ausgeprägt. In Gesprächen im schweriner Wirtschaftsministerium wurde deutlich, daß man die engen Vorgaben der Gemeinschaftsaufgabe sehr begrüßt, da dadurch Ansprüche anderer Ministerien auf Teile der EFRE Gelder abgewehrt werden können. Wie oben bereits 
dargestellt, führen derartige Rahmenbedingungen nicht zu einer Orientierung an den regionalen Präferenzen.

Im Ergebnis muß man feststellen, daß es eher unwahrscheinlich ist, durch eine breite Palette von Fördermaßnahmen die Berücksichtigung regionaler Präferenzen und somit die allokative Effizienz zu erhöhen.

\subsection{Zusammenfassung}

Die Regionalförderung in den neuen Ländern wird im Rahmen der Gemeinschaftsaufgabe gemeinsam von Bund und Ländern geplant und finanziert. Der Bund trägt 50\% der Finanzierung und hat somit auch genauso viele Stimmen wie alle Länder zusammen. Die Ergebnisse der Bund-Länder-Planungen müssen allerdings im Rahmen der Beihilfenkontrolle noch der Kommission zur Genehmigung vorgelegt werden. Die Kommission hat diese Kontrolle auf immer mehr Fördertatbestände ausgedehnt. Die Förderung wirtschaftsnaher Infrastruktur galt bisher nicht als beihilferelevant, seit jüngstem wird jedoch auch sie der Beihilfenkontrollle unterzogen.

Die Zuordnung der Handlungs- und Finanzierungskompetenzen verletzt das Kriterium der fiskalischen Äquivalenz und berücksichtigt die regionalen Präferenzen nur ungenügend. Dies führt zu rent-seeking-Verhalten und somit tendenziell zu einer Ausweitung der Fördergebiete. Es zeigt sich, daß die innere Logik des Systems nur durch die Beihilfenkontrolle der EG durchbrochen werden kann. Sie zwingt Bund und Länder zur Reduzierung der Fördergebiete. Die Gemeinschaft könnte somit gemäß dem Konzept einer ordnungspolitischen Regionalpoltik die Funktion der Regelsetzung- und durchsetzung übernehmen. Da sie jedoch eigene konkrete Ziele und Maßnahmenprogramme verfolgt, kann sie diese Funktion nicht wahrnehmen. Sie ist immer versucht, die allgemeingültigen Regeln für Einzelfallentscheidungen zu verändern.

Die Gemeinschaftsaufgabe verfolgt ein konstruktivistisches wirtschaftspolitisches Konzept, das die regionale Entwicklung nach konkreten Vorstellungen steuern will. Sie basiert auf der Exportbasistheorie, dem Konzept der Wachstumspole und Elementen der neuen Wachstumsmodelle. Die Umsetzung der Regionaltheorien stößt an die erwartbaren Grenzen. Die exportorientierten Industrien werden durch den Radius - in Ostdeutschland liegt dieser bei 30 Kilometern ihres Absatzgebietes abgegrenzt. Diese völlig unzureichende Kriterium führt die Idee der Exportbasistheorie ad absurdum.

Auch die Orientierung an den Wachstumsmodellen ist nicht stringent. Beide Konzepte fordern eine Konzentration der Mittel in dynamischen Zentren, damit die für einen Wachstumsprozeß notwendigen externen Effekte erzeugt werden können. Obwohl das Wachstumsziel als wichtig angesehen wird, sind die ostdeutschen Länder nicht zur Konzentration der Mittel verpflichtet. Dies wird mit Informationsdefiziten und der deshalb nicht möglichen Unterscheidung zwischen 
Förder- und Nichtfördergebieten begründet. Der hehre theoretische Anspruch wird in der Realität nicht umgesetzt.

Bezieht man die Gemeinschaft in die Analyse ein, zeigen sich in der institutionellen Analyse die gleichen Defizite wie bei der Gemeinschaftsaufgabe, verstärkt um den eher zentralistischen Politikansatz der Kommission. Die Mißachtung der fiskalischen Äquivalenz, das dadurch induzierte rent-seeking-Verhalten und die Vernachlässigung der regionalen Präferenzen führen im Ergebnis zu einer völligen Verbürokratisierung und somit tendenziell zu einer Zentralisierung der Handlungskompetenzen.

Die Regionalpolitk der Gemeinschaft ist noch stärker als die Gemeinschaftsaufgabe an einem konstruktivistischen wirtschaftspolitischen Konzept orientiert. Die Kommission erwartet von den Ländern umfangreich begründete Maßnahmenprogramme sowie eine schlüssige Evaluation der Förderung. Hat die Gemeinschaftsaufgabe den Anspruch auf bewußte Planung und Steuerung in bestimmten Bereiche fallengelassen, besteht die Gemeinschaft nach wie vor auf diesem Ansatz.

Die institutionellen Strukturen, die wirtschaftspoltischen Konzepte und die regionaltheoretische Fundierung lassen keinen Raum für ein ordnungspolitisches Konzept der Regionalpolitik. Die Gemeinschaft hätte potentiell die Möglichekit, dem Prinzip des regelgebundenen Wettbewerb zwischen regionalen Einheiten zum Durchbruch zu verhelfen. Da sie jedoch eigene Ziele und Maßnahmenprogramme verfolgt, kann sie diese Funktion nicht mehr ausfüllen. Die politische Ökonomie der bestehenden Verflechtungsstrukturen stabilisiert diese und läßt nur wenig Raum für eine Umorientierung der Regionalpolitik.

\section{Regionalpolitik in Mecklenburg-Vorpommern: Implementation der Gemeinschaftsaufgabe und des EFRE}

Nachdem die Ziele und Instrumente der Regionalpolitik dargestellt und analysiert worden sind, gilt es nunmehr, deren Implementation zu untersuchen. Die Implementation der regionalpolitischen Instrumente obliegt den Ländern. Im Rahmen der Gemeinschaftsaufgabe sind sie für die Benennung von sachlichen und räumlichen Förderschwerpunkten zuständig, sie verwalten und vergeben die Mittel und sind auch für die Kontrolle verantwortlich. Die Basis für die Implementation ist sehr brüchig. Es gibt kein konsistentes Zielsystem und die Rahmenpläne der Gemeinschaftsaufgabe bieten kein schlüssiges Maßnahmenkonzept an. Dem Land Mecklenburg-Vorpommern ist es im wesentlichen freigestellt, ob es seine Förderung am Wachstumsziel orientiert konzentriert oder dem Ausgleichsziel folgend auf die benachteiligten Regionen verteilt. Ausgehend von dieser Situation sind drei Fragen zu klären:

a. Gelingt die Umsetzung der wirtschaftspolitischen Konzepte, die Basis der Regionalprogramme sind? 
b. Orientiert sich die Implementation der Regionalpolitik an den Zielen der Programme?

c. Basiert die Implementation auf den die Regionalpolitik fundierenden Theorien? Lassen sich diese Ansätze in die Praxis umsetzen?

Die Untersuchung teilt sich in zwei Komplexe auf. Im ersten Schritt werden die eher technischen Fragen der Implementation untersucht: Förderbedingungen, das Bewilligungsverfahren und die zur Verfügung stehenden Mittel. Im zweiten Schritt wird dann die Verwendung der Fördergelder dargestellt und analysiert. Abschließend wird auf die Ursachen für den Grad der Zielerreichung eingegangen.

\subsection{Implementation der Regionalpolitik: Programme, Verfahren, Mittel}

\subsubsection{Regionalförderprogramme für Mecklenburg-Vorpommern}

Bestandteil des Rahmenplans ist ein Regionalförderprogramm für jedes Fördergebiet der Gemeinschaftsaufgabe. Im Fall der neuen Länder gibt es für jedes Land ein eigenes Programm, das durch die Länder aufgestellt wird. Wesentliche Bestandteile der Regionalförderprogramme sind eine Analyse der Ausgangslage in dem betreffenden Gebiet, die Darstellung der Förderschwerpunkte und Fördermaßnahmen, die Darstellung anderer regionalpolitischer Maßnahmen außerhalb der Gemeinschaftsaufgabe sowie ein meist sehr kurzer Verwendungsnachweis für das abgelaufene Jahr.

Im Rahmen dieser Analyse sind zunächst die räumlichen und sachlichen Schwerpunkte sowie das Bewilligungsverfahren von Interesse. Abbildung 3 gibt eine Übersicht über die im Rahmen dieser Arbeit wichtigen Festlegungen in den Regionalförderprogrammen von 1991 bis 1994. Mecklenburg-Vorpommern versuchte zu Beginn, die Regionalförderung auf die Problemgebiete zu konzentrieren. Die Entwicklung geeigneter Indikatoren, die die Auswahl dieser Gebiete ermöglichen, ist allerdings aufgrund der unzureichenden statistischen Datenlage erst Ende 1992 möglich. Ab dem 22. Rahmenplan wird ein Unterbeschäftigungsindikator ${ }^{175}$ sowie ein Erreichbarkeitsindikator ${ }^{176}$ zur Abgrenzung der Fördergebiete herangezogen. Die Auswahl der Indikatoren zeigt sehr deutlich die Dominanz des Ausgleichsziels in der Förderpolitik des Landes.

In den Unterbeschaftigungsindikator werden alle Menschen einbezogen, die direkt und indirekt von Arbeitslosigkeit betroffen sind. Neben den in den Arbeitslosenstatistik erfaßten Personen werden Personen in Kurzarbeit, Arbeitsbeschaffungsmaßnahmen, Fortbildung oder Umschulung und Vorruhestand/Altersubergangsgeld berucksichtigt. Die so ermittelte Unterbeschätigung erreicht in Mecklenburg-Vorpommern teilweise bis zu $40 \%$. Vgl. "Zweiundzwanzigster Rahmenplan", a.a.O., S. 78.

Der Erreichbarkeitsindikator bildet die verkehrliche Erschließung und das erreichbare Marktpotential ab. 


\begin{tabular}{|c|c|c|c|c|}
\hline \multicolumn{5}{|c|}{$\begin{array}{c}\text { Abbildung 3 } \\
\text { Regionalförderprogramme Mecklenburg-Vorpommern 1991-1994 }\end{array}$} \\
\hline & 1991 & 1992 & 1993 & 1994 \\
\hline Indikator & $\begin{array}{l}\text { Bevölkerungs- } \\
\text { dichte }\end{array}$ & $\begin{array}{l}\text { Arbeitsmarkt } \\
\text { Wirtschaftskraft } \\
\text { Förderergebnisse } \\
\text { Strukturnachteile }\end{array}$ & $\begin{array}{l}\text { Unterbeschäfti- } \\
\text { gungsindikator } \\
\text { Erreichbarkeits- } \\
\text { indikator }\end{array}$ & $\begin{array}{l}\text { Unterbeschäfti- } \\
\text { gungsindikator } \\
\text { Erreichbarkeits- } \\
\text { indikator }\end{array}$ \\
\hline $\begin{array}{l}\text { räumliche } \\
\text { Schwer- } \\
\text { punkte }\end{array}$ & $\begin{array}{l}\text { 1. Oberzentren } \\
\text { 2. } \text { Mittelzentren } \\
\text { mit Teilfunk- } \\
\text { tion eines } \mathrm{OZ} \\
\text { 3. } \text { Mittelzentren } \\
\text { 4. Unterzentren } \\
\text { mit Teilfunk- } \\
\text { tion eines } \mathrm{MZ}\end{array}$ & $\begin{array}{l}\text { Keine explizite } \\
\text { Benennung von } \\
\text { Schwerpunkten }\end{array}$ & $\begin{array}{l}\text { Förderkulisse } \\
\text { mit drei Förder- } \\
\text { regionen }\end{array}$ & $\begin{array}{l}\text { Förderkulisse } \\
\text { mit drei Förder- } \\
\text { regionen }\end{array}$ \\
\hline $\begin{array}{l}\text { sachliche } \\
\text { Schwer- } \\
\text { punkte }\end{array}$ & $\begin{array}{l}\text { Sachliche } \\
\text { Schwerpunkte } \\
\text { für die Kreise } \\
\text { des Landes, dif- } \\
\text { ferenziert nach } \\
\text { Struk- } \\
\text { turproblemen }\end{array}$ & $\begin{array}{l}\text { Keine explizite } \\
\text { Benennung von } \\
\text { Förderschwer- } \\
\text { punkten }\end{array}$ & $\begin{array}{l}\text { Gew. Wirt- } \\
\text { schaft: } \\
\text { 1. Multiplikator- } \\
\text { effekt } \\
\text { 2. Arbeitsplatz- } \\
\text { effekt } \\
\text { 3. Realisierungs } \\
\text {-zeit } \\
\text { Infrastruktur: } \\
\text { 1. wenige neue } \\
\text { Gewerbegeb. } \\
\text { 2. Technologie- } \\
\text { zentren } \\
\text { 3. Überbetriebli- } \\
\text { che Ausbil- } \\
\text { dung }\end{array}$ & $\begin{array}{l}\text { Gew. Wirt- } \\
\text { schaft: } \\
\text { 1. Multiplikator- } \\
\text { effekt } \\
\text { 2. Arbeitsplatz- } \\
\text { effekt } \\
\text { 3. Realisierungs } \\
\text {-zeit } \\
\text { Infrastruktur: } \\
\text { 1. wenige neue } \\
\text { Gewerbegeb. } \\
\text { 2. Technologie- } \\
\text { zentren } \\
\text { 3. Überbetriebli- } \\
\text { che Ausbil- } \\
\text { dung }\end{array}$ \\
\hline $\begin{array}{l}\text { Sonder- } \\
\text { programm } \\
\text {-gebiete }\end{array}$ & \begin{tabular}{|l|} 
Werftstandorte: \\
Stadt und Land- \\
kreis Rostock, \\
Neubrandenburg, \\
Wismar, Stral- \\
sund, Greifs- \\
wald, Landkreis \\
Wolgast \\
$38,2 \%$ der Be- \\
völkerung \\
\end{tabular} & $\begin{array}{l}\text { Östliche Lan- } \\
\text { desteile: } \\
\text { Drei kreisfreie } \\
\text { Städte, } 12 \text { Land- } \\
\text { kreise }\end{array}$ & entfallen & entfallen \\
\hline
\end{tabular}


Die Förderbedürftigkeit steht im Vordergrund, die Förderwürdigkeit, im Sinne der Förderung wachstumsstarker Regionen mit positiven Ausstrahlungseffekten, kommt nicht zum Tragen.

Im ersten Regionalförderprogramm wird die Bevölkerungsdichte als Indikator genutzt. Allerdings entscheidet sich das Land dann zur schwerpunktmäßigen Förderung der zentralen Orte. Obwohl zu dieser Zeit der Landesraumordnungsplan noch erarbeitet wurde, stimmen die hier genannten Städte im wesentlichen mit den dann später durch die Raumordnung definierten Städten überein. Das Programm faßt die Kreise nach Strukturproblemen zusammen (z.B. Werftstandorte und Tourismusregionen) und empfiehlt für die so entwickelten Regionstypen sachliche Schwerpunkte. ${ }^{177}$ Den besonderen Anpassungsproblemen der Werftstandorte wird mit einem Sonderprogramm Rechnung getragen.

Das Regionalförderprogramm 1991 konzentriert die Förderung in den Schwerpunktorten und versucht, ausgehend von einer groben Regionstypologie, abgestimmte Förderkonzepte zu entwickeln. Dieser Ansatz findet sich in den folgenden Programmen nicht wieder. Allerdings fehlt eine räumliche Differenzierung der Fördersätze. Dies wäre nötig gewesen, um ein Instrument zu haben, die festgelegten Schwerpunkte auch durchzusetzen.

Im Regionalförderprogramm 1992 wurden zwar neue Indikatoren eingeführt, jedoch werden hieraus keine sachlichen und räumlichen Schwerpunkte abgeleitet. Der Schwerpunkt des Sonderprogrammgebietes verlagert sich von den Werftstandorten zu den besonders benachteiligten östlichen Landesteilen. Da gemäß Beschluß des Planungsausschusses maximal $40 \%$ der Bevölkerung in Sonderprogrammgebieten leben dürfen, werden Stadt und Landkreis Wismar und Rostock herausgenommen.

Der Versuch, die Regionalförderung nach einem Konzept zu betreiben, das räumliche und sachliche Schwerpunkte setzt, scheint gescheitert. Ein Grund für dieses Scheitern wird in dem Ausmaß der Strukturprobleme gelegen haben, die es zu bewältigen galt. Die verfügbaren Mittel reichten 1991 nicht aus, die eingegangenen Anträge auf Förderung zu bewilligen. ${ }^{178} \mathrm{Da}$ es keine abgestuften Fördersätze und keine eindeutigen Kriterien für die Umsetzung der räumlichen und sachlichen Schwerpunkte gab, gab es kein Instrument, das Förderkonzept durchzusetzen. Auch 1992 war das statistische Datenmaterial unverändert unzulänglich und somit erschien eine verläßliche Abgrenzung von räumlichen und sachlichen Schwerpunkten unmöglich. Erst 1993 gelang es, mit zwei Indikatoren eine Förderkulisse zu definieren, die das Land in drei Regionen einteilt und für diese Regionen unterschiedliche Fördersätze vorsieht (Abbildung 4). Die Förderregion III, die die höchsten Fördersätze erhält, entspricht weitestgehend dem Sonderprogrammgebiet des Jahres 1992. Allerdings wird diese Abgrenzung durch Aus-

Vgl. „Zwanzigster Rahmenplan", a.a.O., S. 78/79.

Vgl. "Einundzwanzigster Rahmenplan", a.a.O., S. 81. 
nahmeregelungen aufgeweicht. So kann bei gewerblichen Vorhaben mit hohem Struktur- oder Arbeitsplatzeffekt , "... ausnahmsweise der zulässige Höchstfördersatz in jeder Region angewendet werden." ${ }^{179}$ Wann ein solcher Effekt vorliegt, obliegt der Entscheidung der zuständigen Stellen des Landes, d.h., hier bietet sich ein weiter Interpretationsrahmen. Sowohl für die Förderung der gewerblichen Wirtschaft als auch für die Infrastrukturförderung werden sachliche Schwerpunkte definiert. Dieses Konzept behielt auch für 1994 Gültigkeit. Das Ausgleichsziel dominiert die Regionalförderprogramme des Landes. Abgesehen von dem auf die zentralen Orte konzentrierten Regionalförderprogramm 1991 werden keine räumlichen Förderschwerpunkte gebildet. Die Regionalförderprogramme versuchen, das vermeintliche allokative Marktversagen durch eine redistributive Politik zu korrigieren.

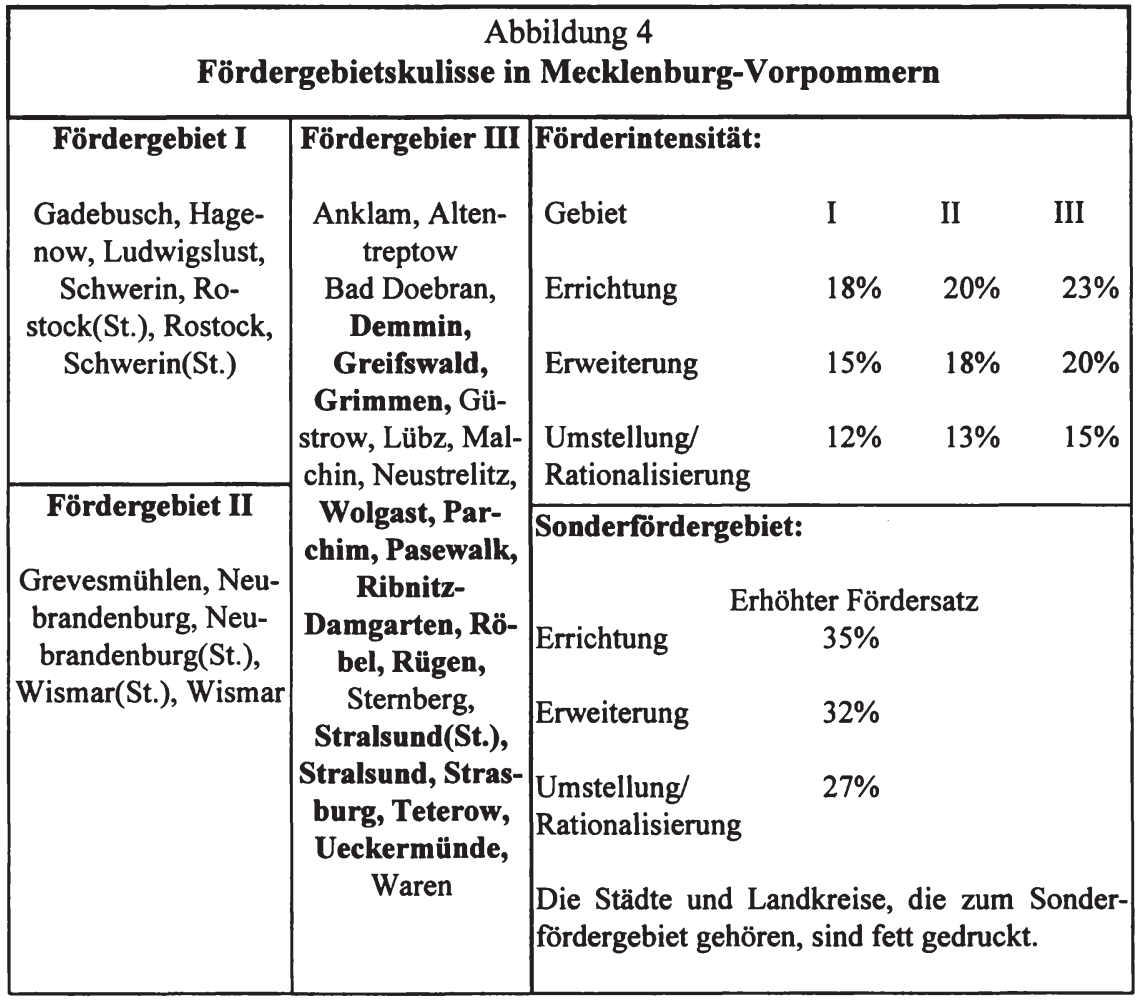


Im Bereich der räumlichen Schwerpunkte gibt es keine Ansätze für gezielte wachstumspolitische Maßnahmen. Lediglich bei den sachlichen Schwerpunkten ist im Ansatz eine Orientierung am Konzept der Wachstums-pole bzw. der neuen Wachstumsmodelle erkennbar. Beides zusammen ergibt jedoch keine stringente Förderstrategie. Die Konzeptionslosigkeit wird noch dadurch verstärkt, daß gemäß dem Rahmenplan die abgeschwächte Form der Exportbasistheorie weiterhin Gültigkeit hat und die Raumordnung sehr dezidiert Schwerpunkte für eine wirtschaftliche Entwicklung definiert hat.

Eine Umsetzung der Ziele und Programme scheitert zunächst an ungenügenden Daten und Informationen. Sie scheitert jedoch im wesentlichen an der politikökonomischen Rationalität. Auch, wenn es unmittelbar einleuchtend ist, daß man im Osten Mecklenburg-Vorpommerns kurz- und mittelfristig keine selbsttragende wirtschaftliche Entwicklung anregen kann, sondern nur in ausgewählten Zentren die Chance hat, Wachstum und damit Annäherung an westdeutsche Standards zu erzielen, ist die Landespolitik nicht in der Lage, diese Erkenntnis in politisches Handeln umzusetzen. Eine bewußte Differenzierung würde vermeintlich zu Stimmenverlusten in den benachteiligten Regionen führen.

\subsubsection{Bewilligungsverfahren}

Für die Bewilligung von Föderanträgen sind in Mecklenburg-Vorpommern grundsätzlich zwei Stellen zuständig. Das Wirtschaftsministerium nahm 1990 und 1991 alle Infrastrukturförderanträge sowie einen Großteil der Föderanträge der gewerblichen Wirtschaft an. Bereits im Laufe des Jahres 1991 nahm das Landesförderinstitut (LFI) seine Tätigkeit auf und übernahm ab 1992 im wesentlichen die Bearbeitung der Förderanträge der gewerblichen Wirtschaft. Die Infrastrukturförderung verblieb in der alleinigen Zuständigkeit des Wirtschaftsministeriums. Das LFI wurde zunächst getragen und aufgebaut durch die Berliner Bank. Nachdem Mecklenburg-Vorpommern sich jedoch für die Norddeutsche Landesbank (Nord LB) als Hausbank entschieden hatte, übernahm die Nord LB auch das LFI. Basierend auf einem Geschäftsbesorgungsvertrag übernimmt die Nord LB für das Land die Antragsbearbeitung und entscheidet in Abstimmung mit dem Land gemäß den gültigen Bedingungen über die Förderanträge. Die enge Kooperation zwischen Wirtschaftsministerium und LFI wird auch durch personelle Verflechtungen deutlich. ${ }^{180}$

Das Antragsverfahren der Gemeinschaftsaufgabe ist unverändert auf die neuen Länder übertragen worden. Neben einem siebenseitigen Antragsformular sind im Bereich der gewerblichen Wirtschaft umfangreiche begründende Unterlagen vorzulegen. Die Beratung der Antragsteller erfolgt in der Regel über die Bank, die die Finanzierung der Investition übernehmen soll. Die Wirtschaftsförderge-

Das Wirtschaftsministerium hat mehrere Mitarbeiter fur Leitungsfunktionen im LFI freigestellt. 
sellschaften spielen nach den Erfahrungen des LFI hierbei nur eine untergeordnete Rolle. Die zentrale Rolle der Banken liegt auch darin begründet, daß sie ihre Finanzierung im wesentlichen von der Zusage der Investitionszuschüsse abhängig machen.

Die Bearbeitungszeiten (Tabelle 5) für Förderanträge in den Jahren 1990-94 spiegeln sehr deutlich die erwartbaren administrativen Schwierigkeiten, Beratungsdefizite sowie die Überforderung der Antragsteller wider. Betrachtet man die in den jeweiligen Jahren eingegangenen Anträge (Infrastruktur und gewerbliche Wirtschaft), so ergibt sich folgendes Bild. Die durchschnittliche Bearbeitungszeit für einen Antrag, der im Jahr 1991 gestellt wurde, lag somit bei 280 Tagen. Trotz erheblich gestiegener Fallzahlen (von 153 auf 947) erhöhte sich die durchschnittliche Bearbeitungszeit für 1991 gestellte Anträge lediglich auf 308 Tage. Trotz sinkender Fallzahlen (821) steigt die Bearbeitungszeit 1992 auf ihren höchsten Stand von 440 Tagen. Ein Grund für diesen Anstieg kann darin liegen, daß noch nicht beschiedene Anträge aus den Vorjahren Kapazität gebunden haben. Erst 1993 tritt bei einem erheblichen Rückgang der Fallzahlen eine deutliche Reduzierung der Bearbeitungszeit ein. Faßt man alle drei Jahre zusammen, so ergibt sich eine durchschnittliche Bearbeitungszeit von 350 Tagen. Mit diesem Wert bildet Mecklenburg-Vorpommern das Schlußlicht in den neuen Bundesländern.

Tabelle 5

\begin{tabular}{|r|r|r|r|}
\hline \multicolumn{5}{|c|}{$\begin{array}{l}\text { Bearbeitungsdauer von Förderanträgen für Mittel der GA } \\
\text { in Mecklenburg-Vorpommern 1990-1994 }\end{array}$} \\
\hline & $\begin{array}{l}\text { Anzahl der } \\
\text { Anträge }\end{array}$ & $\begin{array}{l}\text { Summe der } \\
\text { Bearbeitungstage }\end{array}$ & $\begin{array}{l}\text { durchschnittliche Bear- } \\
\text { beitungszeit in Tagen }\end{array}$ \\
\hline 1990 & 153 & 42900 & 280 \\
\hline 1991 & 947 & 291237 & 308 \\
\hline 1992 & 821 & 361060 & 440 \\
\hline 1993 & 498 & 151519 & 304 \\
\hline gesamt: & 2419 & 846716 & 350 \\
\hline & & & \\
\hline Quelle: Förderdaten des Landes, eigene Berechnungen & \\
\hline
\end{tabular}

Als mögliche Ursache wird von den zuständigen Stellen die große Anzahl kleiner Investitionsprojekte gesehen. Ferner verzögern unvollständig ausgefüllte Anträge und fehlende Unterlagen die Antragsbearbeitung zusätzlich. Leider ist es nicht möglich, die Ursachen für die unverhältnismäßig lange Bearbeitungszeit 
im Rahmen dieser Arbeit genauer zu untersuchen. Die Bündelung der Bearbeitung und Entscheidung von Förderanträgen in einem leistungsfähigen, von einer Bank getragenen Institut sollte eigentlich eine zügigere Bearbeitung zulassen.

\subsubsection{Mittelausstattung der Regionalförderung in Mecklenburg- Vorpommern}

Die Mittel der Regionalförderung im Rahmen der Gemeinschaftsaufgabe und des EFRE werden im Haushalt des Wirtschaftsministeriums in Titel 0603 verbucht. Wie bereits oben erwähnt und aus den folgenden Darstellung ersichtlich, unterliegt die Behandlung der EFRE Mittel Schwankungen, die die Klarheit und Übersichtlichkeit der Angaben erheblich einschränken. Betrachtet man zunächst die Ausgabenseite, so sind hier die Mittel der Gemeinschaftsaufgabe, des Gemeinschaftswerk Aufschwung Ost sowie die Mittel des EFRE für Infrastrukturförderung und gewerbliche Wirtschaft relevant. Für den Vergleich wurden die Haushaltsansätze der jeweiligen Haushaltspläne herangezogen (Tabelle 6). Die Gesamtsumme der Mittel beläuft sich 1991 auf 625.300.000,- DM, steigert sich bis 1994 auf 1.122.353.000,- DM, um dann 1995 auf 875.440.000,- abzusinken. Das Gewicht der unterschiedlichen Instrumente wird allerdings nur eingeschränkt deutlich. Bis 1993 werden die EFRE Mittel in die Gemeinschaftsaufgabe Mittel einbezogen, erst ab 1994 werden sie im Haushalt gesondert ausgewiesen. Nur bei den Mitteln des AO herrscht von Beginn an Klarheit. Um einen Eindruck von der Bedeutung der Instrumente für die Regionalförderung zu bekommen, muß zusätzlich die Einnahmenseite mit betrachtet werden (Tabelle 7). Das Land erhält zweckgebundene Zuweisungen vom Bund für die Gemeinschaftsaufgabe und den $\mathrm{AO}$ sowie von der EG für die EFRE Interventionen.

Grundsätzlich gilt für die Bundesmittel, daß das Land diese kofinanzieren muß, d.h., es muß in gleicher Höhe eigene Gelder bereitstellen. Auch die Gelder der EG müssen mit nationalen Mitteln kofinanziert werden. In der deutschen Praxis werden EFRE Interventionen aus den vorhandenen GA Mitteln kofinanziert. Interventionen, an denen der EFRE beteiligt ist, werden zu 50\% vom EFRE und zu je $25 \%$ von GA Mitteln des Bundes und des Landes abgedeckt. Der Mittelansatz der GA im Landeshaushalt ergibt sich daher aus folgenden Positionen:

- Zuweisungen des Bundes

- Kofinanzierung des Landes (in Höhe der Bundeszuweisungen)

- EFRE Mittel ${ }^{181}$

181 Ab dem Jahr 1994 werden, wie bereits oben erwăhnt, die EFRE Mittel gesondert ausgewiesen. 
Tabelle 6

\begin{tabular}{|c|c|c|c|c|c|c|}
\hline \multicolumn{7}{|c|}{$\begin{array}{c}\text { Mittelansatz der Gemeinschaftsaufgabe, Aufschwung Ost und EFRE } \\
\text { in Mecklenburg-Vorpommern } \\
\text { (Angaben in } 1000 \mathrm{DM})\end{array}$} \\
\hline & 1991 & 1992 & 1993 & 1994 & 1995 & 1996 \\
\hline \multicolumn{7}{|l|}{ GA } \\
\hline Infrastruktur & \begin{tabular}{|l|}
237.650 \\
\end{tabular} & 321.300 & 528.050 & 443.644 & 255.376 & 302.160 \\
\hline $\begin{array}{l}\text { Gewerbliche Wirt- } \\
\text { schaft }\end{array}$ & 237.650 & 321.300 & 528.050 & 443.644 & 383.064 & 453.240 \\
\hline Summe GA & 475.300 & 642.600 & 1.056 .100 & 887.288 & 638.440 & 755.400 \\
\hline \multicolumn{7}{|l|}{ Aufschwung Ost } \\
\hline Infrastruktur & 75.000 & 133.000 & 0 & 0 & 0 & 0 \\
\hline $\begin{array}{l}\text { Gewerbliche Wirt- } \\
\text { schaft }\end{array}$ & 75.000 & 75.000 & 0 & 0 & 0 & 0 \\
\hline $\begin{array}{l}\begin{array}{l}\text { Summe Auf- } \\
\text { schwung. Ost }\end{array} \\
\end{array}$ & \begin{tabular}{|l|}
150.000 \\
\end{tabular} & 208.000 & 0 & 0 & 0 & 0 \\
\hline \multicolumn{7}{|l|}{ EFRE } \\
\hline Infrastruktur & 0 & 0 & 0 & 117.533 & 94.800 & 99.447 \\
\hline $\begin{array}{l}\text { Gewerbliche. Wirt- } \\
\text { schaft }\end{array}$ & 0 & 0 & 0 & 117.533 & 142.200 & 149.171 \\
\hline Summe EFRE & 0 & 0 & 0 & 235.066 & 237.000 & 248.618 \\
\hline $\begin{array}{l}\text { Summe GA,AO, } \\
\text { EFRE }\end{array}$ & \begin{tabular}{|l|}
625.300 \\
\end{tabular} & 850.600 & 1.056 .100 & 1.122 .354 & 875.440 & 1.004 .018 \\
\hline Anteil EFRE & $19 \%$ & $14 \%$ & $11 \%$ & $21 \%$ & $27 \%$ & $25 \%$ \\
\hline
\end{tabular}

Ausgehend von diesen Zahlen läßt sich das Gewicht der verschiedenen Instrumente beurteilen. Der Anteil des EFRE an den regionalpolitischen Ausgaben, die durch Bund, Land und EG mitgetragen werden, sinkt in der ersten Phase von 9193 von $19 \%$ auf $11 \%$. Ab 1994 steigt der Anteil auf 21\% an, beträgt im Jahr $199527 \%$ und $199625 \%$.

Noch deutlicher wird die zunehmende Bedeutung des EFRE, wenn man die $\mathrm{Zu}-$ weisungen betrachtet. Der Anteil des EFRE entwickelt sich von 32\% im Jahr 1991 über 20\% im Jahr 1993 und 47\% im Jahr 1995 auf 44\% in 1996. Die Zu- 
weisungen des EFRE nehmen tendenziell an Gewicht zu, und damit steigt natürlich auch der Einfluß der Kommission auf die Verwendung der Mittel.

Tabelle 7

\begin{tabular}{|c|c|c|c|c|c|c|}
\hline \multicolumn{7}{|c|}{$\begin{array}{c}\text { Zuweisungen von Bund und EG für Mecklenburg-Vorpommern: } \\
\text { Mittelansatz des Haushaltes } \\
\text { (Angaben in } 1000 \mathrm{DM} \text { ) }\end{array}$} \\
\hline & 1991 & 1992 & 1993 & 1994 & 1995 & 1996 \\
\hline \multicolumn{7}{|l|}{ Bundesmittel } \\
\hline GA: Infrastruktur & 89.250 & 113.075 & 235.025 & 221.822 & 127.688 & 151.080 \\
\hline $\begin{array}{l}\text { GA: Gewerbliche } \\
\text { Wirtschaft }\end{array}$ & 89.250 & 113.075 & 235.025 & 221.822 & 191.532 & 226.620 \\
\hline Aufschwung Ost & 75.000 & 104.000 & 0 & 0 & 0 & 0 \\
\hline Summe & 253.500 & 330.150 & 470.050 & 443.644 & 319.220 & 377.700 \\
\hline \multicolumn{7}{|l|}{\begin{tabular}{|l|} 
EG-Mittel \\
\end{tabular}} \\
\hline $\begin{array}{l}\text { Gemeinschafts- } \\
\text { initiativen }\end{array}$ & 0 & 0 & 0 & 0 & 41.034 & 43.020 \\
\hline EFRE & 118.300 & 118.300 & 116.000 & 235.065 & 237.000 & 248.618 \\
\hline Summe & 118.300 & 118.300 & 116.000 & 235.065 & 278.034 & 291.638 \\
\hline \begin{tabular}{|l|} 
Gesamtzuweisunge \\
\end{tabular} & 371.800 & 448.450 & 586.050 & 678.709 & 597.254 & 669.338 \\
\hline Anteil EFRE & $32 \%$ & $26 \%$ & $20 \%$ & $35 \%$ & $47 \%$ & $44 \%$ \\
\hline
\end{tabular}

Durch Veränderungen bei der Mittelbereitstellung im Rahmen der GA gewinnen die EFRE Mittel für die Länder zusätzlich an Bedeutung. Bisher wurden in jedem Jahr mehr Gelder zur Verfügung gestellt als durch bereits bewilligte Anträge aus den Vorjahren gebunden waren. Die Summe der Verpflichtungsermächtigungen, die im laufenden Jahr fällig wurden, war also immer kleiner als die verfügbaren Gelder. In Zukunft sollen in jedem Jahr nur Gelder in Höhe der eingegangenen Verpflichtungsermächtigungen bereitgestellt werden. Das bedeutet, $\mathrm{da}$ aus der GA keine Zuschüsse für Investitionen gewährt werden können, die im laufenden Jahr beantragt und realisiert werden und somit zu einer Auszahlung führen. Bei größeren Investitonsvorhaben mit einem gewissen Planungsvorlauf und einer mehrjährigen Realisierungsphase bereitet diese Regelung keine Probleme. Problematisch wird jedoch die Förderung kleiner und mittlerer Unternehmen, deren Investitionen oftmals recht zügig realisiert werden. In diesen Fällen ist Mecklenburg-Vorpommern dann in Gänze auf die Mittel des EFRE angewiesen, da diese weiterhin abrufbar sind. 
Zum Schluß sei noch auf einen weiteren Aspekt der Mittelausstattung hingewiesen. Aus der Abbildung 11 wird deutlich, daß die Zuweisungen des Bundes im Rahmen der GA erheblichen Schwankungen unterliegen. Der Bund legt seinen Beitrag für die GA jährlich neu fest, so daß die Länder bezüglich der Höhe des Bundeszuschusses keine Planungssicherheit haben. Auch die Finanzierungspläne in den Regionalförderprogrammen, in denen eigentlich die "... für die folgenden Jahre des Planungszeitraums jeweils vorgesehenen Mittel aufgeführt ..." ${ }^{182}$ werden, sind keine sichere Planungsgrundlage. Die EFRE Mittel hingegen sind in ihrer Höhe für den gesamten Programmzeitraum von sechs Jahren in ECU festgelegt. Schwankungen können somit lediglich durch Wechselkursverschiebungen hervorgerufen werden. Diese Mittel sind für die Regionalpolitik der Länder eine feste und kalkulierbare Größe. Die Verteilung der Mittel über die einzelnen Jahre obliegt im wesentlichen den Ländern. Das Land MecklenburgVorpommern hat in den Jahren 1991-93 knapp 120 Millionen DM pro Jahr vom EFRE erhalten und wird für den Zeitraum 1994-99 ca. 240 Millionen DM pro Jahr vom EFRE bekommen. Diese Planungsicherheit verbunden mit der veränderten Mittelbereitstellung im Rahmen der GA dürfte die europäische Regionalpolitik mittelfristig für die Länder sehr interessant machen.

\subsection{Einsatz der Fördermittel}

In den Regionalförderprogrammen des Untersuchungszeitraumes dominiert ganz eindeutig das Ausgleichsziel. Die Förderkulisse, die Maßstab für die Förderung ist, sieht in den schwächsten Regionen die höchsten Fördersätze vor. Aber auch die offiziellen wirtschaftspolitischen Zielvorgaben der Staatskanzlei präferieren das Ausgleichsziel.

Damit ein Ausgleich zwischen stärkeren und schwächeren Regionen des Landes erreicht wird, muß die Intensität der Förderung nach bestimmten Kriterien differenziert werden. Die Regionen des Landes mit einer hohen Förderbedürftigkeit müssen somit eine höhere Förderintensität aufweisen. Als Indikator für die Förderbedürftigkeit wird die durchschnittliche Arbeitslosigkeit im Beobachtungszeitraum herangezogen. Die Förderintensität wird in der Förderung pro Kopf, den Investitionen pro Kopf und dem Fördersatz gemessen.

Nach einem allgemeinen Überblick über die Verwendung der Fördergelder wird im weiteren Verlauf der Analyse die regionale Verteilung der Mittel sowie die Ausrichtung an den Zielen und Schwerpunkten untersucht.

182 $\S 5$ Nr. 3 Gesetz uber die Gemeinschaftsaufgabe Verbesserung der regionalen Wirtschaftsstruktur. 


\subsubsection{Förderung im Rahmen der GA: Ein Überblick}

Die Förderung der GA läßt sich in zwei Bereiche aufteilen: Förderung der gewerblichen Wirtschaft und Förderung der wirtschaftsnahen Infrastruktur. Im Rahmen der GA wurden vom 01.10.1990 bis zum 30.06.1994 insgesamt ca. 3,4 Mrd. DM an Zuschüssen bewilligt. Hiervon entfielen ca. 1,6 Mrd. DM auf den Bereich der wirtschaftsnahen Infrastruktur und 1,8 Mrd. DM auf die Förderung der gewerblichen Wirtschaft.

\section{A. Förderung der gewerblichen Wirtschaft}

Die Verteilung der Förderung im Bereich der gewerblichen Wirtschaft auf die einzelnen Investitionsarten wird in Tabelle 8 verdeutlicht. Der größte Anteil der Mittel (38\%) wurde für die Förderung der Errichtung eines Gewerbebetriebes bewilligt. Zweitwichtigste Investitionsart war der Erwerb eines Gewerbebetriebes. Hierauf entfielen 27\% der Mittel. Am unbedeutendsten war die Förderung von Unternehmensverlagerungen. Auf sie entfallen lediglich 4\% der Mittel.

Im Durchschnitt wurden die Investitionen mit $18 \%$ gefördert. Die höchsten Fördersätze wurden für Errichtung und Erwerb bewilligt, am geringsten wurde die Rationalisierung mit 12\% gefördert. Der Arbeitsplatzeffekt der Förderung wird durch die mit Hilfe der Förderung gesicherten bzw. zusätzlich geschaffenen Arbeitsplätze dargestellt. Die Zahlen beruhen auf den Angaben der Antragsteller. Da der Arbeitsplatzeffekt ein Kriterium für die Höhe der Förderung ist, wird die Einhaltung der Zusagen durch das Landesförderinstitut kontrolliert. Dies ist bei der Schaffung zusätzlicher Arbeitsplätze durchaus möglich, die Angaben über gesicherte Arbeitsplätze sind jedoch nur sehr bedingt kontrollierbar.

Der Arbeitsplatzeffekt hat allerdings nur eine eingeschränkte Aussagefähigkeit. Dies bezieht sich sowohl auf die Anzahl der angegebenen Arbeitsplätze als auch auf Ursächlichkeit der Förderung für ihren Erhalt. Ob z.B. durch eine Erweiterungsinvestition alle bisher bestehenden Arbeitsplätze gesichert werden, ist durchaus fraglich. Erfolgt die Investition nicht, werden sicherlich nicht alle bestehenden Arbeitsplätze vernichtet. Ferner ist unklar, ob die Investition ohne Förderung nicht trotzdem - eventuell in eingeschränktem Umfang - vorgenommen würde. ${ }^{183}$ Die Förderung hätte dann nur den Teil der Arbeitsplätze gesichert, der im Fall ohne Förderung weggefallen wäre.

183 Es gibt unterschiedliche empirische Befunde zu dieser Frage. Eine Modellrechnung von Recker für die Zeit 1973-77 ergibt, daß ca. 83\% der geforderten Arbeitsplătze auch ohne die Förderung entstanden wăren (Vgl. Harald Jürgensen: "Raumwirtschaft II: Politik", a.a.O., S. 438). Die Investitionsförderung in den neuen Lăndern bewirkt bei $42,8 \%$ der Unternehmen, daß die Investition durchgefuhrt werden kann (Vgl. Ifo Studien zur Finanzpolitik "Die Effizienz der finanzpolitischen Fordermaßnahmen in den neuen Bundeslăndern", a.a.O., S. 139). 
Tabelle 8

\begin{tabular}{|l|r|r|r|r|r|r|r|}
\hline \multicolumn{7}{|l|}{ Förderung der gewerblichen Wirtschaft vom 01.10.1990 - 30.06.1994 in Mecklenburg-Vorpommern } \\
\hline & Errichtung & Erwerb & Erweiterung & Umstellung & Rationalis. & Verlagerung & gesamt \\
\hline & & & & & & & \\
\hline & & & & & & \\
\hline & 3.336 .024 & 2.279 .837 & 1.292 .157 & 41.948 & 2.467 .829 & 435.976 & 9.853 .771 \\
\hline Föderfähige Invest. & 697.668 & 496.025 & 234.908 & 7.899 & 312.552 & 76.613 & 1.825 .665 \\
\hline Anzahl der Projekte & 1.113 & 276 & 806 & 19 & 181 & 301 & 2.696 \\
\hline Anteil der Mittel & $38 \%$ & $27 \%$ & $13 \%$ & $0 \%$ & $17 \%$ & $4 \%$ & $100 \%$ \\
\hline Anteil der Invest. & $34 \%$ & $23 \%$ & $13 \%$ & $0 \%$ & $25 \%$ & $4 \%$ & $100 \%$ \\
\hline Fördersatz & $20,91 \%$ & $21,76 \%$ & $18,18 \%$ & $18,83 \%$ & $12,67 \%$ & $17,57 \%$ & $18,53 \%$ \\
\hline & & & & & & & \\
\hline Arbeitsplätze & & & & & & & \\
\hline gesichert & 972 & 8.881 & 14.705 & 206 & 21.123 & 3.561 & 49.448 \\
\hline zusätzlich & 19.154 & 12.148 & 8.098 & 490 & 2.449 & 3.342 & 45.681 \\
\hline gesamt & 20.126 & 21.029 & 22.803 & 696 & 23.572 & 6.903 & 95.129 \\
\hline Anteil der Arbpl. & $21 \%$ & $22 \%$ & $24 \%$ & $0 \%$ & $25 \%$ & $7 \%$ & $100 \%$ \\
\hline Förderung pro Arbpl. & 34.665 & 23.588 & 10.302 & 11.349 & 13.259 & 11.098 & 19.192 \\
\hline Quelle: Förderstatistik des Landes sowie eigene Berechnungen & & & \\
\hline
\end{tabular}


Unklar ist die Berücksichtigung von Arbeitsplätzen, die im Zuge einer Investition wegfallen. Insbesondere bei der Rationalisierung von Betrieben, die in den durch alte Industriestrukturen geprägten Regionen eine weit überdurchschnittliche Bedeutung hat, werden bestehende Arbeitsplätze vernichtet. Dieser negative Arbeitsplatzeffekt wird teilweise in der Statistik der GA ausgewiesen. Es ist allerdings nicht nachvollziehbar, ob dies durchgängig in allen Fällen geschehen ist.

Unter Berücksichtigung dieser Einschränkungen läßt sich feststellen, daß der größte Anteil des Arbeitsplatzeffektes durch Rationalisierungs- und Erweiterungsinvestitionen erzielt wird. Errichtung und Erwerb haben einen nur unwesentlich geringeren Effekt.

Ein Blick auf die Förderung pro Arbeitsplatz macht die Investitionskosten für die Schaffung bzw. Sicherung eines Arbeitsplatzes deutlich. Am höchsten sind die Investitionen erwartungsgemäß bei der Errichtung eines Betriebes. Hier ist auch der Anteil der zusätzlich geschaffenen Arbeitsplätze besonders hoch $(90,6 \%)$. Das durchschnittliche förderfähige Investitionsvolumen von ca. $170.000 \mathrm{DM}$ pro Arbeitsplatz liegt nur wenig unter den durchschnittlichen Investitionen von ca. 200.000 DM pro neu geschaffenem Arbeitsplatz in Westdeutschland. Die Kapitalintensität der neugeschaffenen Arbeitsplätze in Mecklenburg-Vorpommern entspricht somit westdeutschen Standards.

Zum Abschluß möchte ich noch auf eine Unsicherheit bezüglich der Förderung von Rationalisierungsinvestitionen hinweisen. Ermittelt man die durchschnittliche förderfähige Investitionssumme bei der Errichtung bzw. Rationalisierung eines Gewerbebetriebes, ergeben sich erhebliche Unterschiede. Im Durchschnitt werden für Errichtungsinvestitionen ca. 3 Mill. DM pro Projekt investiert. Für Rationalisierungsinvestitionen werden ca. 14 Mill. DM pro Projekt aufgewendet. Rationalisierungsinvestitionen werden somit vornehmlich in großen Betrieben durchgeführt. Die meisten großen Betriebe mit mehr als 1000 Beschäftigten gab es 1991 in Mecklenburg-Vorpommern im Bereich des Schiffbaus. ${ }^{184}$ Insbesondere in diesem Bereich hat es vielfältige sektorale Förderungen im Rahmen der Privatisierung durch die THA gegeben. Die Förderung im Rahmen der GA macht für die Werften nur einen Bruchteil der Gesamtförderung aus. ${ }^{185}$ Bezieht man die in diesem Rahmen gewährte Förderung auf die gesicherten Arbeitsplätze, so ergibt sich eine Förderung von über 500.000,- DM pro Arbeitsplatz. Wollte man die tatsächliche Höhe der Förderung von Rationalisierungsinvestitionen pro Ar-

184

185

Vgl. Statistisches Landesamt Mecklenburg-Vorpommern: "Statistisches Jahrbuch 1993", Schwerin 1993, S. 148.

Insgesamt wurden fur die Weiterfuhrung und Privatisierung der Werften 6,2 Mrd. DM aufgewendet. Für die Privatisierung der MTW-, Warnow- und Peene-Werft sind hiervon nach EG Kriterien 2,8 Mrd. DM beihilferechtlich relevant. Vgl. Karl Lichtblau: "Privatisierungs- und Sanierungsarbeit der Treuhandanstalt", 1993, S. 36-42. 
beitsplatz ermitteln, müßte man den Teil der o.a. Hilfen hinzurechnen, der der Rationalisierung der Werften diente. Da hierfür allerdings das notwendige Datenmaterial fehlt, läßt sich lediglich feststellen, daß die tatsächliche Förderung pro Arbeitsplatz bei Rationalisierungsinvestitionen erheblich über dem nach der GA Statistik ermittelten Wert liegt.

\section{B. Förderung der wirtschaftsnahen Infrastruktur}

Im Bereich der wirtschaftsnahen Infrastruktur gibt es 17 förderfähige Investitionsarten, von denen zehn zum Bereich der touristischen Infrastruktur gehören. ${ }^{186}$ Von den verbleibenden sieben Investitionsarten sind vier wegen ihrer quantitativen bzw. qualitativen Bedeutung explizit dargestellt (Tabelle 9) ${ }^{187}$

Tabelle 9

\section{Förderung der wirtschaftsnahen Infrastruktur vom 01.10.1990 - 30.06.1994 in Mecklenburg-Vorpommern}

(Angaben in $1000 \mathrm{DM}$ )

\begin{tabular}{|l|r|r|r|r|r|}
\hline & $\begin{array}{c}\text { Förderfähige } \\
\text { Investitionen }\end{array}$ & $\begin{array}{r}\text { Bewilligte } \\
\text { Mittel }\end{array}$ & $\begin{array}{c}\text { Prozent der } \\
\text { Gesamt- } \\
\text { mittel }\end{array}$ & $\begin{array}{c}\text { Prozent der } \\
\text { Gesamtinv. }\end{array}$ & Fördersatz \\
\hline Gewerbegebiete & 1.050 .203 & 739.747 & $47 \%$ & $49 \%$ & $70,44 \%$ \\
\hline Verkehrsverb. & 427.461 & 363.292 & $23 \%$ & $20 \%$ & $84,99 \%$ \\
\hline Bildung & 73.266 & 52.357 & $3 \%$ & $3 \%$ & $71,46 \%$ \\
\hline F\&E Zentren & 93.958 & 84.353 & $5 \%$ & $4 \%$ & $89,78 \%$ \\
\hline Tourist. Infrastr. & 358.334 & 267.214 & $17 \%$ & $17 \%$ & $74,57 \%$ \\
\hline sonstiges & 121.803 & 79.511 & $5 \%$ & $6 \%$ & $65,28 \%$ \\
\hline & & & & & \\
\hline Summe & 2.125 .024 & 1.586 .473 & & & \\
\hline Quelle: Förderstatistik des Landes, eigene Berechnungen & & \\
\hline
\end{tabular}

Der weitaus größte Anteil der Mittel (46\%) floß in die Erschließung bzw. Restaurierung von Gewerbegebieten. Die Förderung von Verkehrsverbindungen konnte $20 \%$ der Mittel binden, knapp 17\% standen für die touristische Infra-

186 Zur touristischen Infrastruktur gehören: Seebrücken, Strandpromenaden, Parks, Tierparks, Wander- und Fahrradwege, Erholungszentren, Schwimmbåder, Toiletten, TouristInfo-Center und sonstige Maßnahmen.

187 Unter die Rubrik „sonstige Investitionen“ fällt die Förderung von Energie- und Wasserversorgung, Abwasser- und Abfallbeseitigungsanlagen sowie die Errichtung von Gewerbezentren. 
struktur zur Verfügung. Für Technologiezentren und für Bildungseinrichtungen wurden nur ein sehr geringer Anteil der Gelder eingesetzt. Die Fördersätze schwanken von $65,28 \%$ für sonstige Investitionen bis zu $89,78 \%$ für Technologiezentren. Im Durchschnitt betrug der Fördersatz 74,66\%.

Die Förderung von Gewerbeflächen konzentrierte sich auf die Erschließung neuer Flächen "auf der grünen Wiese". Ungeklärte Eigentumsverhältnisse und fehlende Planungsgrundlagen zwangen die Städte und Gemeinden zu diesem Weg. Das für die Genehmigung der Förderanträge zuständige Wirtschaftsministerium stand zunächst vor einem Informationsproblem. Weder der Bestand an vorhandenen Gewerbeflächen noch der Bedarf neuer Flächen sowie das insgesamt notwendige Angebot waren bekannt. ${ }^{188}$ Der Bedarf an Gewerbefläche pro Beschäftigtem schwankt von 50 bis 500 qm und ist abhängig von der Branche, der Produktionstechnik und planungsrechtlichen Vorgaben. Für Mecklenburg-Vorpommern wurde zunächst ein Richtwert von 300 qm pro Erwerbstätigem bzw. $30 \mathrm{qm}$ pro Einwohner den Berechnungen zu Grunde gelegt. Betrachtet man die Ausgangslage, so ergab sich in weiten Teilen des Landes eine Unterversorgung mit Gewerbeflächen. Lediglich in zehn Kreisen stand genügend Fläche pro Einwohner zur Verfügung. Allerdings fehlte es dem Land an abschließenden Informationen über die Verfügbarkeit der Flächen. Neben ungeklärten Eigentumsverhältnissen und Altlasten kann die Lage bzw. neue städteplanerische Vorgaben die Verfügbarkeit der Flächen einschränken. Für die weiteren Betrachtungen werden diese Flächen daher nur zu 50\% berücksichtigt. Mit Stand Ende 1992 ergab sich für die Altflächen zuzüglich der geförderten Bruttofläche folgendes Bild (vergl. Tabelle 10).

In 22 von 31 Kreisen lag die Ausstattung mit Gewerbeflächen unterhalb des Richtwertes von 30 qm pro Einwohner. Bezieht man die nicht geförderten Flächen mit ein, so erreichen lediglich zwölf Kreise nicht den geforderten Richtwert. Diese Werte sagen nichts über die tatsächliche Nachfrage nach Gewerbefläche aus und können daher auch nur sehr eingeschränkt als Nachweis einer Über- bzw. Unterversorgung mit Gewerbefläche angesehen werden. Eine effiziente Steuerung der Gewerbeflächenförderung wäre daher nur dann möglich, wenn das Land Kenntnisse über die tatsächliche bzw. erwartete Nachfrage in den Regionen hätte. Diese Informationen liegen dem Land jedoch nicht vor und können auch nur unter hohem administrativem Aufwand beschafft und verarbeitet werden. In den Regionen gibt es zumindest sichere Informationen über die aktuelle Nachfrage und die Preise, die Investoren zu zahlen gewillt sind. Auch die Fähigkeit der Regionen, die zukünftige Nachfrage nach Gewerbeflächen abzuschätzen, dürfte zumindestens genauso gut sein wie die des Landes. 
Tabelle 10

\section{Gewerbeflächenbestand in Mecklenburg-Vorpommern 1992}

(Angaben in qm pro Einwohner)

\begin{tabular}{|l|c|l|l|c|}
\hline & $\begin{array}{c}\text { 1/2 der Altfläche } \\
\text { plus Bruttoförder- } \\
\text { fläche }\end{array}$ & & $\begin{array}{c}\text { 1/2 der Altfläche } \\
\text { plus neue Gewer- } \\
\text { begebiete }\end{array}$ \\
\hline Altentreptow & 3 & & Wolgast & 15 \\
\hline Wolgast & 11 & & Altentreptow & 15 \\
\hline Gadebusch & 12 & & Grevesmühlen & 16 \\
\hline Röbel/Müritz & 13 & & Gadebusch & 16 \\
\hline Neustrelitz & 17 & & Röbel/Müritz & 20 \\
\hline Bützow & 18 & & Strasburg & 21 \\
\hline Grevesmühlen & 18 & & Neustrelitz & 23 \\
\hline Bad Doberan & 19 & & Bützow & 24 \\
\hline Strasburg & 19 & & Lübz & 24 \\
\hline Ückermünde & 20 & & Neubrandenburg & 26 \\
\hline Rügen & 21 & & Waren & 26 \\
\hline Lübz & 22 & & Ückermünde & 29 \\
\hline Stralsund & 22 & & Pasewalk & $\mathbf{3 0}$ \\
\hline Neubrandenburg & 23 & & Anklam & $\mathbf{3 0}$ \\
\hline Güstrow & 26 & & Rostock & $\mathbf{3 2}$ \\
\hline Waren & 26 & & Stralsund & $\mathbf{3 3}$ \\
\hline Pasewalk & 26 & & Schwerin & $\mathbf{3 3}$ \\
\hline Schwerin & 27 & & Rügen & $\mathbf{3 3}$ \\
\hline Hagenow & 27 & Sternberg & $\mathbf{3 4}$ \\
\hline Parchim & 28 & & Demmin & $\mathbf{3 4}$ \\
\hline Ludwigslust & 28 & & Güstrow & $\mathbf{3 6}$ \\
\hline Demmin & 28 & & Bad Doberan & $\mathbf{3 6}$ \\
\hline Wismar & $\mathbf{3 1}$ & & Wismar & $\mathbf{4 0}$ \\
\hline Rostock & $\mathbf{3 1}$ & & Parchim & $\mathbf{4 1}$ \\
\hline Greifswald & $\mathbf{3 3}$ & & Ribnitz-Dammg. & $\mathbf{4 2}$ \\
\hline Ribnitz-Dammg. & $\mathbf{3 3}$ & & Greifswald & $\mathbf{4 4}$ \\
\hline Sternberg & $\mathbf{3 4}$ & & Grimmen & $\mathbf{5 0}$ \\
\hline Teterow & $\mathbf{3 6}$ & & Malchin & $\mathbf{6 1}$ \\
\hline Anklam & $\mathbf{3 7}$ & & Hagenow & $\mathbf{6 2}$ \\
\hline Grimmen & $\mathbf{3 7}$ & & Ludwigslust & $\mathbf{6 2}$ \\
\hline Malchin & $\mathbf{6 0}$ & & Teterow & $\mathbf{7 9}$ \\
\hline Quelle: Landeswirtschaftsministerium & & \\
\hline & & & \\
\hline
\end{tabular}

In dieser durch unzureichende Informationen gekennzeichneten Situation liegt die Vermutung nahe, daß der hier verfolgte zentrale Planungs- und Steuerungs- 
prozeß zu Fehlallokationen führt. Diese Vermutung wird noch durch weitere institutionelle Rahmenbedingungen unterstützt.

Das Kriterium der fiskalischen Äquivalenz wird auch in diesem Bereich nicht erfüllt. Die Förderung der Gewerbeflächen beträgt im Durchschnitt $70 \%$ des Investitionsvolumens. Die Kommune hat somit nur noch einen sehr geringen Eigenanteil der Investition zu tragen. Die Kosten der Investition fallen überwiegend auf der Landesebene an. Der Nutzen eines Gewerbegebietes fallt vorwiegend auf kommunaler bzw. regionaler Ebene an. Die Ansiedlung von Gewerbebetrieben sollte zu steigenden Gewerbesteuer- und höheren Einkommenssteuereinnahmen führen. Daß diese Erwartungen teilweise nicht kurzfristig, sondern erst mittel- bis langfristig erfüllbar sind, blieb bei den Entscheidungen allerdings oftmals unberücksichtigt. Auch in diesem Bereich ist zu vermuten, daß durch das Auseinanderfallen von Kosten und Nutzen eines Gewerbegebietes, eine Fehlallokation stattfindet. Weitere institutionelle Rahmenbedingungen unterstützen die Fehlallokation von Fördergeldern. Die Honorare der Planer werden nach Größe der Gewerbefläche berechnet. Dies verleitet in der Tendenz zu einer überdimensionierten Erschließung von Gewerbeflächen.

Die Erschließung und Vermarktung von Gewerbeflächen durch private Investoren ${ }^{189}$ war nur sehr eingeschränkt möglich. Die Gemeinde mußte, damit eine Förderung möglich wurde, Eigentümer der Fläche sein. ${ }^{190}$ Dies führte dazu, daß die Kommunen, die keine geeigneten eigenen Flächen hatten, neue Flächen hinzukauften. Durch diese Regelung wurde eine private, marktgerechte ErschlieBung von Flächen, die den Kommunen das Planungs- und Investitionsrisiko genommen hätte, verhindert. Erst ab dem zweiundzwanzigsten Rahmenplan waren Erschließungen auch dann förderungsfähig, "... wenn die Grundstücke nicht im Eigentum der Gemeinde ..." ${ }^{191}$ standen. Zu diesem Zeitpunkt war der größte Teil der Förderanträge für die Erschließung von Gewerbeflächen allerdings bereits gestellt.

Auffällig ist, daß ein nur sehr geringer Anteil der Fördergelder für den Bereich Bildung eingesetzt wurde. Diese Zahl macht die starke Sachkapitalorientierung der Förderung deutlich. Die Förderung des Humankapitals durch den Ausbau bzw. die Schaffung von Bildungseinrichtungen bindet lediglich 3,3\% der Mittel. Bildungseinrichtungen sind öffentliche Güter mit besonders hohen externen Effekten. Die Träger dieser Einrichtungen müssen bereit und in der Lage sein, die Folgekosten einer solchen Einrichtung zu tragen. Hierzu sind in der Regel nur

189 Dies ist z.B. bei der Erschließung von Bauland im Rahmen von Vorhaben und ErschlieBungsplänen ein găngiges Verfahren. Der Investor uberplant, erschließt und vermarktet eine Fläche auf eigene Kosten.

Dies galt fur den zwanzigsten und einundzwanzigsten Rahmenplan (91-94 und 92-95).

Zweiundzwanzigster Rahmenplan, a.a.O., S. 26. 
Kammern, Verbände, Gewerkschaften und andere öffentliche Institutionen bereit. Da diese Institutionen in Mecklenburg-Vorpommern finanziell wie organisatorisch noch nicht die gleiche Leistungsfähigkeit wie in Westdeutschland haben, wäre in diesem Bereich zumindestens ein zeitlich befristetes Engagement des Landes notwendig gewesen.

\subsubsection{Schwerpunkte der Regionalförderung}

Basis für diese Untersuchung sind die 36 Kreise und kreisfreien Städte des Landes Mecklenburg-Vorpommern, die bis zur Kreisgebietsreform am 01.07.1994 Bestand hatten. Der Beobachtungszeitraum umfaßt somit alle Förderanträge, die vom 03.10.90 bis zum 30.06.1994 genehmigt wurden. Dieses Vorgehen war notwendig, da ein Umrechnen der Förderdaten, der Förderkulisse sowie der Indikatoren für die Förderbedürftigkeit auf die neue Kreisstruktur nicht möglich war.

Als Kriterium für die Förderbedürftigkeit wird die durchschnittliche Arbeitslosenquote auf Kreisebene von 1992 bis 1994 gewählt. Für die Ermittlung eines Unterbeschäftigungsindikators auf Kreisebene fehlen wichtige statistische Daten. ${ }^{192}$ Ferner läßt sich dieser Indikator lediglich für die Jahre '93 und '94 ermitteln. Das gleiche Problem ergibt sich für den Erreichbarkeitsindikator.

Die Arbeitslosigkeit ist ein guter Indikator für die Förderbedürftigkeit einer Region. Überdurchschnittliche Arbeitslosigkeit über mehrere Jahre hinweg zeigt, $\mathrm{da} ß$ in Wachstumsphasen unterdurchschnittliche Arbeitsplatzzuwächse und in Rezessionsphasen überdurchschnittliche Arbeitsplatzverluste realisiert werden. Der Grad der Fähigkeit, Arbeitsplätze zu schaffen bzw. zu halten, zeigt die Leistungsfähigkeit einer Wirtschaft.

Somit ist die Förderbedürftigkeit in den Regionen Mecklenburg-Vorpommerns am höchsten, die im Durchschnitt der letzten Jahre eine überdurchschnittliche Arbeitslosigkeit hatten. Die Regionen mit einer unterdurchschnittlichen Arbeitslosigkeit weisen eine leistungsstärkere Wirtschaftsstruktur auf. Auf diese Regionen müßte sich eine Förderung konzentrieren, die sich am Wachstumsziel orientiert. Leider sind die Arbeitslosenzahlen nur für drei Jahre statistisch vergleichbar, da die Erhebung erst ab 1992 nach den westdeutschen Methoden erfolgen konnte.

Die Förderintensität wird am Fördersatz, an der Förderung pro Kopf sowie an den Investitionen pro Kopf gemessen. Der Indikator Förderung pro Kopf ist besonders aussagefähig, da er die absolut in eine Region geflossene Förderung berücksichtigt. Der Fördersatz ${ }^{193}$ sagt etwas über die Höhe des Anreizes aus, mit

So sind z.B. nicht alle Daten der arbeitsmarktpolitschen Maßnahmen auf Kreisebene vorhanden. Eine Umrechnung ist nur möglich, wenn man mit Schätzungen arbeitet, die die Aussagefăhigkeit des Indikators allerdings beeintrăchtigen. 
dem Investitionen im Rahmen der Programmimplementation in bestimmte Regionen gelenkt werden sollen.

Das vorhandene Datenmaterial läßt keine Zeitreihenuntersuchung zu und erlaubt es auch nicht, eine Evaluation des Fördermitteleinsatzes, im Sinne einer Untersuchung der Wirkungen, durchzuführen. Im Rahmen einer Querschnittsanalyse ist es möglich, zu überprüfen, ob die Zielvorgaben der Reginalförderprogramme im Rahmen der Implementation eingehalten worden sind. Tabelle 11 zeigt die regionale Verteilung der Fördermittel gemäß den o.a. Kriterien. Zur Verdeutlichung ist die Tabelle entsprechend der ab 1993 geltenden Förderkulisse gegliedert. Ferner sei noch darauf hingewiesen, daß die kreisfreien Städte Schwerin, Rostock, Neubrandenburg, Wismar, Greifswald und Stralsund für die statistische Analyse mit ihrem jeweiligen Umlandkreis eine Region bilden. Dieses Verfahren ist notwendig, weil die Arbeitslosenzahlen nur in dieser Form vorliegen. Neben der statistischen Notwendigkeit spricht jedoch auch die starke ökonomische und politische Verflechtung der Städte mit ihren Umlandkreisen für dieses Verfahren. Der Fördersatz für gewerbliche Investitionen schwankt zwischen $34,47 \%$ in Parchim und 9,17\% in Wismar. Im Landesdurchschnitt wird ein Fördersatz von 18,5\% erreicht. Der extrem hohe Fördersatz in Parchim ist aus dem zugänglichen Datenmaterial nicht erklärbar. Parchim gehört zu den Regionen, in denen die Förderhöchstsätze der GA in Ausnahmefällen überschritten werden dürfen. Obwohl in diesem Fall für Errichtungsinvestitionen Subventionen in Höhe von $35 \%$ der förderfähigen Investitionen zulässig sind, erklärt dies nicht die Tatsache, daß über einen Zeitraum von fast vier Jahren und über alle Investitionsarten hinweg ein Fördersatz von fast 35\% erreicht wird. Der niedrige Fördersatz für Wismar läßt sich durch die Dominanz der Rationalisierungsinvestitionen erklären, in die fast $68 \%$ der Fördermittel flossen. Hierbei handelt es sich vornehmlich um die Rationalisierung der Werften, die, wie oben bereits dargestellt, im wesentlichen durch Beihilfen außerhalb der GA gefördert wird. Da auch für diese Förderung der Höchstsatz von $35 \%$ gilt, steuert die GA im Fall Wismar bei Rationalisierungsinvestitionen lediglich 7,43\% bei. Ähnlich ist die Situation in Stralsund gelagert, das auch einen weit unterdurchschnittlichen Fördersatz aufweist. Hier entfallen $61 \%$ der Mittel auf Rationalisierungsinvestitionen, die mit einem Fördersatz von $11 \%$ subventioniert werden.

Betrachtet man die Fördersätze in den drei Förderregionen, so kann man keine Orientierung am Ausgleichsziel erkennen. Die Förderregion III wird nur geringfügig stärker gefördert als die Förderregion I. Berücksichtigt man die Tatsache, daß der Fördersatz in der Region II durch die Zahlen aus Wismar nach unten verzerrt ist, ist anzunehmen, daß auch diese Region etwa entsprechend dem Landesdurchschnitt gefördert wurde. Das Ergebnis der Regressionsanalyse bestätigt diesen Eindruck. Es gibt keinen signifikanten Zusammenhang zwischen der Arbeitslosenquote und dem Fördersatz. Es ist dem Land nicht gelungen, dort die 
stärksten Anreize zu gewähren, wo die Förderbedürftigkeit am größten ist. Allerdings orientiert sich die Förderung auch nicht am Wachstumsziel, da es keine signifikante Beziehung zwischen Arbeitslosigkeit und Fördersatz gibt. Die tatsächliche Gestaltung der Fördersätze weicht somit von den Vorgaben der Regionalförderprogramme deutlich ab.

Tabelle 11

\section{Föderintensität in Mecklenburg-Vorpommern vom 01.10 .1990 - 30.06.1994}

(Förderregion 1 und 2)

\begin{tabular}{|c|c|c|c|c|c|c|}
\hline & $\begin{array}{l}\text { Förder- } \\
\text { satz } \\
\text { (Gew.) }\end{array}$ & \begin{tabular}{|l|} 
Förd. \\
pro Kopf \\
$($ Gew $)$
\end{tabular} & \begin{tabular}{|l|} 
Invest. \\
pro Kopf \\
(Gew.)
\end{tabular} & \begin{tabular}{|l|} 
Förd. \\
pro Kopf \\
(Infra.)
\end{tabular} & $\begin{array}{l}\text { Förd. } \\
\text { pro Kopf } \\
\text { (ges.) }\end{array}$ & $\begin{array}{l}\text { Arbeits- } \\
\text { losen- } \\
\text { quote * }\end{array}$ \\
\hline $\begin{array}{l}\text { Schwerin } \\
\text { (ST/L) }\end{array}$ & 19 & 1.014 & 5.247 & 303 & 1.317 & $12,1 \%$ \\
\hline $\begin{array}{l}\text { Rostock } \\
\text { (ST/L) }\end{array}$ & 18 & 1.263 & 7.016 & 956 & 2.219 & $14,7 \%$ \\
\hline Rostock(ST) & 18 & 1.221 & 6.943 & 890 & 2.111 & \\
\hline Schwerin (ST) & 19 & 1.062 & 5.505 & 261 & 1.322 & \\
\hline Gadebusch & 21 & 583 & 2.808 & 140 & 724 & $14,0 \%$ \\
\hline Hagenow & 19 & 1.614 & 8.708 & 474 & 2.088 & $12,5 \%$ \\
\hline Ludwigslust & 20 & 1.042 & 5.299 & 419 & 1.462 & $16,2 \%$ \\
\hline Schwerin (L) & 20 & 845 & 4.334 & 454 & 1.299 & \\
\hline Rostock (L) & 20 & 1.530 & 7.482 & 1.375 & 2.906 & \\
\hline FördReg1 & 19 & 1.189 & 6.407 & 639 & 1.828 & \\
\hline $\mathrm{NBB}(\mathrm{ST} / \mathrm{L})$ & 18 & 941 & 5.136 & 741 & 1.682 & $15,6 \%$ \\
\hline Wismar (ST/L) & 11 & 997 & 9.280 & 639 & 1.635 & $18,0 \%$ \\
\hline NBB (ST) & 22 & 874 & 4.002 & 520 & 1.394 & \\
\hline Wismar (ST) & 9 & 1.184 & 12.919 & 621 & 1.805 & \\
\hline Grevesm. & 20 & 754 & 3.731 & 757 & 1.511 & $17,1 \%$ \\
\hline NBB (L) & 13 & 1.163 & 8.892 & 1.473 & 2.636 & \\
\hline Wismar (L) & 21 & 682 & 3.190 & 669 & 1.351 & \\
\hline FördReg2 & 15 & 930 & 6.389 & 707 & 1.637 & \\
\hline MVP gesamt & 19 & 981 & 5.303 & 854 & 1.834 & $17,8 \%$ \\
\hline
\end{tabular}


Tabelle 11 (Fortsetzung)

\begin{tabular}{|c|c|c|c|c|c|c|}
\hline \multicolumn{7}{|c|}{$\begin{array}{c}\text { Föderintensität in Mecklenburg-Vorpommern } \\
\text { vom 01.10.1990 - 30.06.1994 } \\
\text { (Förderregion 3) } \\
\end{array}$} \\
\hline & \begin{tabular}{|l|} 
Förder- \\
satz \\
(Gew.)
\end{tabular} & \begin{tabular}{|l|} 
Förd. \\
pro Kopf \\
(Gew)
\end{tabular} & \begin{tabular}{|l|} 
Invest. \\
pro Kopf \\
(Gew.)
\end{tabular} & \begin{tabular}{|l|} 
Förd. \\
pro Kopf \\
(Infra.) \\
\end{tabular} & \begin{tabular}{|l|} 
Förd. \\
pro Kopf \\
(ges.)
\end{tabular} & $\begin{array}{l}\text { Arbeits- } \\
\text { losen- } \\
\text { quote * }\end{array}$ \\
\hline $\begin{array}{l}\text { Greifswald } \\
\text { (ST/L) }\end{array}$ & 20 & 374 & 1.892 & 643 & 1.017 & $16,4 \%$ \\
\hline Strals. (ST/L) & 14 & 1.125 & 8.063 & 1.451 & 2.576 & $18,2 \%$ \\
\hline Greifsw. (ST) & 19 & 429 & 2.212 & 791 & 1.220 & \\
\hline Strals. (ST) & 13 & 1.349 & 10.025 & 1.341 & 2.690 & \\
\hline Altentreptow & 21 & 70 & 328 & 511 & 581 & $22,6 \%$ \\
\hline Anklam & 20 & 1.606 & 8.071 & 1.294 & 2.900 & $25,1 \%$ \\
\hline Bad Dober. & 20 & 893 & 4.481 & 956 & 1.849 & $19,6 \%$ \\
\hline Bützow & 21 & 455 & 2.191 & 288 & 744 & $18,3 \%$ \\
\hline Demin & 16 & 402 & 3.262 & 757 & 1.159 & $24,9 \%$ \\
\hline Greifswald (L) & 22 & 228 & 1.035 & 247 & 475 & \\
\hline Grimmen & 22 & 456 & 2.072 & 376 & 832 & $21,2 \%$ \\
\hline Güstrow & 20 & 1.327 & 6.729 & 548 & 1.875 & $19,2 \%$ \\
\hline Lübz & 21 & 1.230 & 5.980 & 382 & 1.612 & $19,5 \%$ \\
\hline Malchin & 20 & 1.273 & 6.321 & 1.115 & 2.388 & $22,3 \%$ \\
\hline Neustrelitz & 22 & 464 & 2.088 & 791 & 1.256 & $18,0 \%$ \\
\hline Parchim & 34 & 1.488 & 4.316 & 723 & 2.211 & $19,0 \%$ \\
\hline Pasewalk & 21 & 388 & 1.827 & 546 & 934 & $22,8 \%$ \\
\hline Ribnitz Dam. & 22 & 1.242 & 5.629 & 1.995 & 3.236 & $21,8 \%$ \\
\hline Röbel/Mür. & 21 & 820 & 3.829 & 1.081 & 1.901 & $21,5 \%$ \\
\hline Rügen & 22 & 957 & 4.267 & 1.617 & 2.575 & $19,7 \%$ \\
\hline Sternberg & 20 & 503 & 2.515 & 790 & 1.294 & $20,9 \%$ \\
\hline Stralsund (L) & 21 & 447 & 2.143 & 1.784 & 2.231 & \\
\hline Straßburg & 23 & 557 & 2.461 & 952 & 1.509 & $21,2 \%$ \\
\hline Teterow & 21 & 333 & 1.594 & 968 & 1.302 & $21,8 \%$ \\
\hline Ueckerm. & 23 & 711 & 3.087 & 1.109 & 1.820 & $25,0 \%$ \\
\hline Waren & 20 & 1.013 & 5.037 & 750 & 1.764 & $21,3 \%$ \\
\hline Wolgast & 17 & 1.200 & 7.135 & 1.598 & 2.798 & $20,7 \%$ \\
\hline FördReg3 & 20 & 876 & 4.436 & 1.008 & 1.884 & \\
\hline MVP gesamt & 19 & 981 & 5.303 & 854 & 1.834 & $17,8 \%$ \\
\hline
\end{tabular}


Betrachtet man die Förderung pro Kopf im Bereich der gewerblichen Wirtschaft, so ergibt sich genau das Gegenteil dessen, was gemäß dem Ausgleichsziel zu erwarten gewesen wäre. Auf Ebene der Förderregionen fällt die Förderung pro Kopf von 1189,- DM in der Region I über 930,- DM in der Region II auf 876,- DM in der Region III ab. Diese Zahlen sprechen nicht für die Orientierung am Ausgleichsziel, sie legen vielmehr den Schluß nahe, daß die Förderung pro Kopf am Wachstumsziel orientiert ist, da die Region mit der geringsten Förderbedürftigkeit die höchste Förderung pro Kopf erhält. Das Ergebnis der Regressionsanalyse bestätigt diese Vermutung jedoch nicht. Auch in diesem Fall gibt es keinen signifikanten Zusammenhang zwischen Arbeitslosigkeit und der Förderung pro Kopf. Die Ergebnisse der Analyse können auch bei diesem Indikator keine Ausrichtung am Ausgleichsziel belegen, dem Land ist es nicht gelungen, in die Regionen mit der höchsten Bedürftigkeit mehr Fördermittel zu leiten als in die weniger bedürftigen Regionen. Die statistische Analyse läßt jedoch auch keine Ausrichtung an einem anderen wirtschaftspolitischen Ziel erkennen.

Als letzten Indikator, der sich auf die Förderung der gewerblichen Wirtschaft bezieht, werden die geförderten Investitionen pro Kopf betrachtet. Die geförderte Pro-Kopf-Investition betrug in der Region I 6407,- DM, in der Region II 6389,- DM und in der Region III 4436,- DM. Auch hier läßt sich keine Orientierung am Ausgleichsziel ablesen. Die Regressionsanalyse ergibt auch für diesen Indikator keine signifikante Beziehung zur Arbeitslosigkeit. Die Förderbedürftigkeit hat somit keinen Einfluß auf die Verteilung der geförderten Investitionen.

Im Bereich der Infrastrukturförderung gibt es auf Ebene der Förderregionen geringfügige Unterschiede bei den Fördersätzen. In der Region I werden öffentliche Infrastrukturinvestitionen mit durchschnittlich $71 \%$ gefördert, in der Region II mit 74,5\% und in der Region III mit 75,6\%. Betrachtet man den wesentlich aussagefähigeren Indikator der Förderung pro Kopf, ist ein deutlicher Schwerpunkt in der Region III zu erkennen. In die Region I fließen mit 639,- DM pro Kopf die wenigsten Mittel. Die Region II erhält 707,- DM pro Kopf und die Region III 1008,- DM. Die Regressionsanalyse bestätigt diese Tendenz jedoch nicht. Es gibt keinen signifikanten Zusammenhang zwischen der Arbeitslosigkeit und der Infrastrukturförderung pro Kopf. Selbst in dem Bereich, in dem die staatliche Steuerung der Mittelverwendung am leichtesten möglich wäre, scheitert die Umsetzung der regionalpolitischen Zielvorgaben. Das Land scheint nicht in der Lage zu sein, die beschlossenen Grundsätze in wirtschaftspolitische Taten umzusetzen.

Faßt man zum Abschluß die Förderung der gewerblichen Wirtschaft und die Infrastrukturförderung zusammen, ergibt sich für die Gesamtförderung pro 
Kopf folgendes Bild. Die Region I erhält 1827,- DM pro Kopf, die Region II 1637,- DM und die Region III 1884,- DM. Auch diese Zahlen lassen keine eindeutige Orientierung am Ausgleichsziel erkennen, die Ergebnisse der Regressionsanalyse bestätigen diesen Eindruck. Auch der letzte Indikator bestätigt die Orientierungslosigkeit der Mittelverwendung.

Die Ergebnisse der statistischen Analyse sind eindeutig. Es ist keine bewußte Orientierung an einer bestimmten Förderkonzeption erkennbar, die Mittel scheinen vielmehr nach anderen als ökonomischen Maßstäben verteilt worden zu sein.

\subsubsection{Förderung der zentralen Orte}

Die Vorgaben der Landesplanung und die sachlichen Schwerpunkte der Regionalförderprogramme weisen den zentralen Orten eine besondere Funktion zu und verlangen, daß bestimmte Aktivitäten in diesen Orten konzentriert werden. Auch aus Sicht der Regionaltheorie spielen die zentralen Orte eine wichtige Rolle. Sie können Wachstumspole darstellen und nach der neuen Wachstumstheorie mögliche Standorte für kommunikative Netzwerke sein. Aufgrund dieser besonderen Bedeutung gilt die folgende Analyse der Struktur und Intensität der Förderung in den zentralen Orten.

Die Landesplanung unterscheidet in fünf Oberzentren $(\mathrm{OZ})$, elf Mittelzentren (MZ) und acht Mittelzentren mit Teilfunktion (MZTF). In den Oberzentren werden $15,74 \%$ der Mittel für Technologie und Gründerzentren aufgewandt. Dies sind $81 \%$ der Fördermittel, die hierfür in Mecklenburg-Vorpommern eingesetzt wurden. Die Förderung von Gewerbegebieten spielt mit 25\% der Mittel hingegen nur eine untergeordnete Rolle. Die Aufwendungen für Bildungsinvestitionen liegen geringfügig über dem Landesdurchschnitt. Von den landesweit hierfür verausgabten Mitteln entfallen 35\% auf die Oberzentren. In den Mittelzentren liegt der Anteil der Technologieförderung mit 4,4\% unter dem Landesdurchschnitt, der Anteil der Förderung von Gewerbegebieten (48,5\%) und Bildungseinrichtungen (6,7\%) über dem Landesdurchschnitt.

Bei den Mittelzentren mit Teilfunktion spielen Technologie- und Gründerzentren keine Rolle mehr. Hier dominiert eindeutig die Förderung von Gewerbegebieten mit $54 \%$ der Mittel. Die Bildungsförderung liegt nur geringfügig über dem Landesdurchschnitt.

Faßt man alle zentralen Orte zusammen, so binden sie $98,46 \%$ der Fördermittel für Technologie- und Gründerzentren und 73,5\% der Mittel für Bildungseinrichtungen. Im Bereich der Gewerbegebietsförderung werden allerdings nur 38,9\% der Mittel durch die zentralen Orte gebunden. Die Förderintensität, gemessen in den Fördersätzen, liegt im Infrastrukturbereich leicht über dem Landesdurchschnitt und im gewerblichen Bereich leicht unter dem Landesdurchschnitt. Im Ergebnis läßt sich an Hand dieser Analyse feststellen, daß sich die zentralen Orte bezüglich der Förderintensität nicht wesentlich vom Landesdurchschnitt 
entfernen. Aufgrund der vorhergehenden Analysen war auch kein anderes Ergebnis zu erwarten.

Tabelle 12

\begin{tabular}{|c|c|c|c|}
\hline \multicolumn{4}{|c|}{$\begin{array}{c}\text { Förderung von Oberzentren, } \\
\text { Mittelzentren und Mittelzentren mit Teilfunktionen in } \\
\text { Mecklenburg-Vorpommern vom 01.10.1990 - 30.06.1994 } \\
\text { (Angaben in Prozent oder 1000 DM) }\end{array}$} \\
\hline & $\begin{array}{l}\text { Technologie- } \\
\text { förderung }\end{array}$ & $\begin{array}{l}\text { Gewerbege- } \\
\text { biete }\end{array}$ & Bildung \\
\hline Förderung $\mathrm{OZ}$ & \begin{tabular}{|r|}
68.642 \\
\end{tabular} & 113.296 & 18.359 \\
\hline Anteil a.d. Ges.Förd. & $81 \%$ & $15 \%$ & $35 \%$ \\
\hline Förderung MZ & 12.645 & 107.418 & 14.872 \\
\hline Anteil a.d. Ges. Förd. & $15 \%$ & $15 \%$ & $28 \%$ \\
\hline Förderung MZTF & 1.766 & 67.062 & 5.252 \\
\hline Anteil a.d. Ges.Förd. & $2 \%$ & $9 \%$ & $10 \%$ \\
\hline Summe zentr. Orte & 83.053 & 287.776 & 38.484 \\
\hline Anteil der zentr. Orte & $98 \%$ & $39 \%$ & $74 \%$ \\
\hline Gesamtförderung & 84.353 & 739.747 & 52.357 \\
\hline \multicolumn{4}{|c|}{$\begin{array}{l}\text { Fördersatz und Verteilung der Mittel bei OZ, MZ und MZTF } \\
\text { in Mecklenburg-Vorpommern 01.10.1990-30.06.1994 }\end{array}$} \\
\hline & \begin{tabular}{|r|}
$\mathbf{O Z}$ \\
\end{tabular} & $\mathbf{M Z}$ & MZTF \\
\hline \multicolumn{4}{|l|}{ Technologie } \\
\hline Fördersatz & $90,00 \%$ & $90,00 \%$ & $82,80 \%$ \\
\hline Anteil a.d. Förderung & $15,74 \%$ & $44,00 \%$ & $14,10 \%$ \\
\hline \multicolumn{4}{|l|}{ Gewerbegebiete } \\
\hline Fördersatz & $71,00 \%$ & $74,00 \%$ & $70,40 \%$ \\
\hline Anteil a.d. Förderung & $25,00 \%$ & $48,50 \%$ & $53,86 \%$ \\
\hline \multicolumn{4}{|l|}{ Bildung } \\
\hline Fördersatz & $61,55 \%$ & $84,87 \%$ & $74,54 \%$ \\
\hline Anteil a.d. Förderung & $4,20 \%$ & $6,70 \%$ & $4,21 \%$ \\
\hline \multicolumn{4}{|l|}{ Infrastruktur gesamt } \\
\hline Fördersatz & $78,80 \%$ & $76,74 \%$ & $75,47 \%$ \\
\hline \multicolumn{4}{|l|}{ Gewerbl. Wirtschaft } \\
\hline Fördersatz & $17,60 \%$ & $17,10 \%$ & $17,74 \%$ \\
\hline
\end{tabular}


Eine Orientierung am Wachstumsziel und eine überdurchschnittliche Förderung von potentiellen Wachstumszentren wäre mit den oben dargestellten Ergebnissen nicht vereinbar gewesen.

Hinsichtlich der Struktur der Förderung ist insbesondere bei den OZ und MZ ein deutlicher Unterschied zum Landesdurchschnitt erkennbar. Die Infrastruktur für Technologie- und Gründerzentren sowie für Bildungseinrichtungen konnte in den zentralen Orten konzentriert werden. Dies entspricht voll und ganz den Vorgaben der Regionalplanung und kann als Basis für die Entstehung kommunikativer Netzwerke in den zentralen Orten angesehen werden. Bei der Förderung von Gewerbeflächen ist die Konzentration der Mittel auf die zentralen Orte nicht so deutlich gelungen. Allerdings liegen, bezogen auf die geforderte Fläche, $52 \%$ der Gewerbegebiete in zentralen Orten. ${ }^{194}$ Nach Einschätzung des Landes lag der Schwerpunkt für die Förderung neuer Gewerbegebiete ab 1993 eindeutig auf den $\mathrm{OZ}$ und $\mathrm{MZ}$, so daß zu erwarten ist, daß die Konzentration dieser Mittel in den zentralen Orten in den folgenden Jahren zunehmen wird.

\subsection{Umsetzung der regionalpolitischen Ziele und Programme}

Ziel der Politik des Landes war, die Förderung am Ausgleichsziel zu orientieren, d.h., in den Regionen mit hoher Arbeitslosigkeit eine hohe Förderintensität zu erzielen. Dieses Ziel ist nicht erreicht worden. Die Vorgabe der Regionalförderprogramme, den Schwerpunkt der Förderung in die benachteiligten Regionen Mecklenburg-Vorpommerns zu legen, ist nicht eingehalten worden. Die statistische Analyse gibt auch keine Hinweise auf eine bewußte Orientierung an einem anderen wirtschaftspolitischen Ziel. Zumindest läßt sich bezogen auf den Indikator Arbeitslosigkeit keine bewußte Konzentration der Mittel in dynamischen und stärker prosperierenden Regionen erkennen. Die Verteilung der Mittel scheint anderen als ökonomischen Kriterien gefolgt zu sein.

Das der Regionalpolitik zu Grunde liegende wirtschaftspolitische Konzept hat sich als nicht umsetzbar erwiesen. Das Land geht von einem Marktversagen aus, das durch eine redistributive Förderpolitik ausgeglichen werden soll. Alle regionalpolitischen Programme fordern eine bewußte und zielgerichtete Planung und Steuerung der wirtschaftlichen Entwicklung, die sich an einem bestimmten regionalpolitischen Leitbild orientieren soll. In der Umsetzung der Förderung ist jedoch keine bewußte Orientierung an einem konkreten wirtschaftspolitischen Konzept mehr zu erkennen. Die geforderte und geplante bewußte Steuerung hat nicht stattgefunden, die Förderung muß sich demnach an anderen Kriterien orientiert haben.

Aber auch eine Orientierung an den regionalökonomischen Theorien ist kaum erkennbar. Das Land stand verkürzt gesagt vor zwei Alternativen: Förderung des Wachstums im Lande oder Ausgleich für benachteiligte Regionen. Wachstums-

Vgl. interner Vermerk des Wirtschaftsministeriums vom 03.09.1992. 
förderung hätte eine Stärkung Mecklenburg-Vorpommerns im Vergleich zum übrigen Bundesgebiet gebracht, mit der Gefahr, die Disparitäten innerhalb Mecklenburg-Vorpommerns zu vergrößern. Die meisten regionalökonomischen Theorien, auf denen die bundesdeutsche Regionalpolitik basiert, fordern eine Konzentration der Mittel, um in dynamischen ökonomischen Zentren selbsttragende Wachstumsprozesse zu induzieren. Das Land hat sich offiziell für eine auf Ausgleich gerichtete Politik entschieden. De facto läßt sich weder dies noch das Gegenteil in der Realität der Förderung erkennen. Die konkrete Förderpraxis scheint sich nicht an Regionaltheorien orientiert zu haben.

Da sich für den Außenstehenden kein schlüssiges Konzept hinter der Verteilung der Fördergelder erkennen läßt, ist abschließend zu klären, welche Gründe zu dieser Situation geführt haben. Es ist nicht nur ein einzelner Faktor, der die starken Abweichungen von der geplanten Förderpolitik erklärt, allerdings dominieren die politik-ökonomischen Gründe.

\section{a. Unberücksichtigte Zielkonflikte}

Die Zielkonflikte zwischen Wachstums- und Ausgleichsziel wurden nicht berücksichtigt. Der Forderung der Raumordnung nach Konzentration von Infrastruktur- und Unternehmensinvestitionen in zentralen Orten (vornehmlich in Mittel- und Oberzentren) stand der Wunsch der Landesregierung nach einer am Ausgleichsziel orientierten Förderung gegenüber. Bei dem Versuch, beide Ziele zu erreichen, hat sich kein Ziel durchsetzen können, so daß der Eindruck der Konzeptionslosigkeit entstehen muß.

\section{b. Unzureichende theoretische Fundierung der Förderung}

Die regionaltheoretischen Modelle sind nicht in der Lage, die regionale wirtschaftliche Entwicklung zu erklären. Die vorhandenen Modelle können nur Teilaspekte erklären und bleiben in entscheidenden Fragen unbestimmt. Weder ist die Auswahl der exportorientierten Industrie theoretisch gelöst, noch sind die Branchen ermittelbar, die die so wichtigen positiven externen Effekte erzeugen. Die Umsetzung der Theorien in wirtschaftspolitisches Handeln ist damit nur sehr schwer möglich. Allerdings stehen alle wirtschaftlichen Organisationen vor diesem Problem. Keinem Unternehmen, daß eine Produkt- und Absatzplanung macht, nimmt die moderne Betriebswirtschaftslehre das unternehmerische Risiko ab. In einem ordnungspolitischen Konzept der Regionalpolitik steht das Land mit seiner wirtschaftspolitischen Konzeption im Wettbewerb mit anderen Regionen. Im Rahmen dieses Konzeptes wäre keine Strategie zu haben, weil man keine hundertprozentige Sicherheit über die Ergebnisse hat, der falscheste Weg. 


\section{c. Mangelnde Operationalisierung}

Die Zielvorgaben der regionalen Wirtschaftspolitik sowie der Regionalförderprogramme waren nicht hinlänglich operationalisiert. In den Jahren 1991 und 1992 gab es weder Indikatoren zur Abgrenzung der Fördergebiete noch klar definierte Differenzierungen der Fördersätze. Für die Jahre 1993 und 1994 gab es zwar eine Förderkulisse, die jedoch keine signifikanten Auswirkungen auf die regionale Verteilung der Mittel hatte. Die Gründe hierfür lagen und liegen in der Interpretationsfähigkeit der Vorgaben sowie den Ausnahmeregelungen der Förderprogramme. Die zuständigen Stellen haben dadurch einen gewissen Spielraum bei der Entscheidung über Förderanträge. Eine Überschreitung der regional zulässigen Fördersätze kann aus Sicht des Landes z.B. dann notwendig sein, wenn Unternehmen, die für die Region bzw. für das Land eine besondere Bedeutung haben, die Förderhöhe zur Bedingung für ihre Investition machen. Diese sicherlich notwendige Flexibilität kann dann allerdings dazu führen, daß die ursprünglich gesetzten räumlichen Schwerpunkte verfehlt werden.

\section{d. Verteilung der Förderung ist von Anträgen der Unternehmen und Kommunen abhängig}

Eine weitere denkbare Erklärung könnte in der regional unterschiedlichen Nachfrage nach Förderung gesehen werden. Die Schwerpunkte der Förderung lägen dann in den Regionen, aus denen die meisten Anträge kommen. Das Land hat, insbesondere im Bereich der gewerblichen Wirtschaft, nur sehr begrenzte Einflußmöglichkeiten auf Anzahl und Volumen der Förderanträge. Die Regionalförderung wird als Angebot an Unternehmen und Kommunen verstanden, und die Verteilung wird durch die Nachfrage bestimmt.

Bezüglich der Förderung pro Kopf bzw. der Investitionen pro Kopf ist dieser Erklärungsansatz durchaus schlüssig. Allerdings vermag er nicht, die regionale Differenzierung der Fördersätze zu erklären. Könnte die Nachfrage der gewerblichen Wirtschaft nach Förderung deren regionale Verteilung bestimmen, so ist anzunehmen, daß die meisten Anträge aus den Regionen kämen, die aus Sicht der Unternehmen eine höhere Standortqualität haben. Einziges Selektionskriterium für die Bewilligung von Fördergeldern wären dann die Vorgaben der Landesplanung, die eine Konzentration der wirtschaftlichen Aktivitäten in den zentralen Orten vorsehen. Für den Bereich der gewerblichen Wirtschaft würde dies zu einer stärkeren Orientierung am Wachstumsziel führen.

Im Bereich der Infrastrukturförderung läßt sich das Ergebnis eines solchen Verfahrens nicht so eindeutig bestimmen. $\mathrm{Da}$ in den neuen Ländern der Bedarf an wirtschaftsnaher Infrastruktur in allen Landesteilen sehr groß ist, sind grundsätzlich aus allen Regionen Förderanträge zu erwarten. Die Anforderungen an die Ausstattung mit wirtschaftsnaher Infrastruktur sind jedoch in zentralen Regionen mit einem hohen wirtschaftlichen Aktivitätsniveau größer als in periphe- 
ren Regionen mit geringer wirtschaftlicher Aktivität. Ferner fordert die Landesplanung, das Infrastrukturangebot in den zentralen Orten zu konzentrieren. Somit wäre auch für die Infrastrukturförderung in der Tendenz eine stärkere Konzentration der Mittel in den zentralen Orten zu erwarten.

Da das Land dem Ausgleichsziel Vorrang vor dem Wachstumsziel gibt, wäre es nur konsequent, im Rahmen der Implementation der Förderung diesen inhärenten Konzentrationstendenzen entgegenzuwirken. Die tatsächliche regionale Verteilung der Fördermittel ließe sich damit erklären, daß das Land die Konzentration der Mittel in den wirtschaftlich stärkeren Zentren zwar verhindern konnte, jedoch nicht in der Lage war, eine Verteilung der Mittel gemäß dem Ausgleichsziel durchzusetzen.

\section{e. Förderwettbewerb mit anderen Ländern}

Der Wirtschaftsstandort Mecklenburg-Vorpommern hat im Wettbewerb um Investitionen eine Vielzahl von Konkurrenten, die auch in den Genuß von Fördermitteln kommen. Neben den neuen Ländern sind dies vornehmlich Standorte, die in Förderregionen der europäischen Gemeinschaft liegen. Bei ansonsten gleichwertigen Bedingungen kann ein Unternehmen seine Standortentscheidung von der Höhe der Förderung abhängig machen. Wenn das Land solch ein Unternehmen nicht an einen Konkurrenten verlieren will, muß es den Forderungen nachgeben. Dies kann im Ergebnis zu einer Aufweichung der Förderkulisse und somit zu einer Verschiebung der Schwerpunkte führen. An diesem Phänomen zeigt sich sehr deutlich, wie bedeutsam die Existenz von allgemeinverbindlichen Regeln für eine ordnungspolitische Regionalpolitik ist. Die Regionen befinden sich im Wettbewerb um mobile Produktionsfaktoren und haben das Recht, zur Verbesserung ihrer wirtschaftlichen Situation konkrete Fördermaßnahmen durchzuführen. Dies geschieht jedoch nicht im Rahmen bindender allgemeinverbindlicher Regeln. Die Länder haben einen Spielraum, die Regeln zu differenzieren und somit von Fall zu Fall über die Höhe der Fördersätze zu entscheiden. Eine regelgebundene Regionalpolitik, die direkte Unternehmensförderungen zuließe, würde die Förderung in allen Regionen an gleiche Regeln binden. So wäre dann ein Wettbewerb der wirtschaftspolitischen Konzepte und Strategien und der immobilen Standortfaktoren um die mobilen möglich. In der hier vorliegenden Situation führt das Fehlen einer Regelbindung zu massivem rentseeking-Verhalten und damit zu einer Fehlallokation öffentlicher Mittel.

\section{f. Politik-ökonomische Gründe}

Im Kern läßt sich das Problem der Regionalpolitik auf politik-ökonomische Gründe zurückführen. Zunächst werden die wirtschaftspolitischen Konzepte und regionalwirtschaftlichen Theorien zur politischen Legitimation von Förderprogrammen benutzt. Insbesondere die Gemeinschaft und der Bund vertreten eine 
Regionalpolitik, die, basierend auf einem konstruktivistischen wirtschaftspolitischen Verständnis, eine theoretisch fundierte Planung und Steuerung regionaler Entwicklungen verfolgt. Die politischen Planer unterstellen bei ihren Planungen Informationen über Wirkungszusammenhänge, die sie nicht haben. Obwohl diese theoretischen Konzepte weder auf europäischer noch auf bundesdeutscher Ebene umsetzbar sind, werden sie zur Legitimation einer politisch motivierten Umverteilung benutzt. Die Geschichte der GA und des EFRE belegen dies eindeutig. Auf der Ebene des föderalen Systems, auf der nach dem Konzept einer ordnungspolitischen Regionalpolitik, die regionalen Organisationen bewußt Ziel-Mittel orientierte Maßnahmenprogramme umsetzen könnten, ist der gleiche Mechanismus zu beobachten. Das Land begründet seine Regionalpolitik mit Marktversagen und legitimiert damit die Konzentration der Fördergelder in den schwachen Regionen. Diese Strategie läßt sich gegenüber den regionalen politischen Akteuren jedoch nicht durchhalten. Landtagsabgeordnete gewinnen an Bedeutung und Gewicht in ihrer Region, wenn sie Fördergelder beschaffen können. Bei knappen Mehrheiten in Ausschüssen und im Landtag hat der einzelne Abgeordnete ein erhebliches Verhandlungsgewicht gegenüber der bewilligenden Landesregierung. Dieses Gewicht ist um so größer, je bedeutsamer die Region aus Sicht der Regierung für den Erhalt der Regierungsmehrheit in kommenden Wahlen ist. Diese politischen Einflüsse spielen sich auch auf der kommunalen Ebene ab. Jeder Bürgermeister wird für seine Stadt um Zuschüsse und Subventionen kämpfen und er wird gegenüber der bewilligenden Landesregierung gute Gründe haben, warum es in ihrem Interesse ist, seine Stadt nicht zu vernachlässigen. Neben diesen offiziellen Einflußfaktoren spielen die parteipolitischen Machtsicherungsmechanismen eine Rolle. Der Abgeordnete kann nur dann wiedergewählt werden, wenn die Partei ihn nominiert. Der Minister wird nur dann erneut im Parlament vertreten sein, wenn seine regionale Parteiorganisation noch hinter ihm steht. Es gibt genügend politik-ökonomische Beweggründe, von der angestrebten Verteilung der Gelder abzuweichen, um im Sinne einer politischen Nutzenmaximierung einen Erhalt der politischen Macht zu gewährleisten. Auch hier zeigt sich erneut die Chance, die ein regelgebundener Wettbewerb bietet. Wenn die Regeln für alle gleich sind, dann gibt es keinen Raum für derartige „Bargaining Prozesse“. Dann können sich die regionalen politischen und wirtschaftlichen Kräfte auf die Gestaltung der Standortbedingungen konzentrieren, die sich dann auch an den regionalen Präferenzen orientieren könnten.

\subsection{Zusammenfassung}

Die Umsetzung der Förderprogramme in detaillierte landesbezogene Planungen erfolgt im Rahmen von Regionalförderprogrammen. Die Analyse dieser Programme zeigt, daß sich Mecklenburg-Vorpommern für eine Ausrichtung seiner Förderung am Ausgleichsziel entscheidet. Basierend auf einem konstruktivistischen wirtschaftspolitischen Konzept ist, ausgehend von der Annahme des allo- 
kativen Marktversagens, eine Konzentration der Mittel in den förderbedürftigen Regionen vorgesehen.

Im Untersuchungszeitraum vom 03.10 .1990 bis zum 30.06 .1994 wurden ca. 3,4 Mrd. DM Fördergelder bewilligt. Eine Analyse der Mittelherkunft zeigt sehr deutlich die verflochtenen Strukturen. Im Falle einer Mitfinanzierung durch die Gemeinschaft trägt der EFRE 50\%, der Bund 25\% und das Land die verbleibenden 25\%. Die Mittel aus dem EFRE werden für das Land insbesondere ab 1994 immer bedeutsamer, da sie in den Jahren 94 bis 96 einen Anteil von 35\% bis $44 \%$ der Zuweisungen für regionalpolitische Programme ausmachen.

Die Mittel werden in etwa zu gleichen Teilen auf die Förderung der gewerblichen Wirtschaft und die Förderung der wirtschaftsnahen Infrastruktur verteilt. Es gelingt dem Land nicht, die tatsächliche Förderung an den Vorgaben der Regionalförderprogramme zu orientieren. Die statistische Analyse läßt keine signifikante Beziehung zwischen der Förderintensität und der Förderbedürftigkeit erkennen. Die Umsetzung des wirtschaftspolitischen Konzeptes scheitert, eine bewußte Steuerung der Förderung ist nicht erkennbar. Auch die regionalwirtschaftlichen Theorien, die zur theoretischen Fundierung der Programme herangezogen wurden, lassen sich in der Verteilung der Fördergelder nicht erkennen. So ist weder eine Ausrichtung am Konzept der Wachstumspole noch an den neuen Wachstumsmodellen nachweisbar. Die Verteilung der Förderung scheint somit anderen als ökonomischen Gründen zu folgen.

Die abschließende Analyse der Gründe für die massive Verletzung von Zielen und Vorgaben der Programme zeigt verschiedene Ursachen auf. So spielen theoretische Defizite, wie die unberücksichtigten Zielkonflikte und die mangelnde Operationalisierung der Ziele eine Rolle. Ferner könnte im Implementierungsprozeß die Verteilung der Anträge eine Rolle gespielt haben. Auch der Förderwettbewerb des Landes mit anderen Regionen ist von Bedeutung. Da das System rent-seeking-Verhalten der Unternehmer fördert, versuchen diese, höhere Förderkonditionen mit dem Hinweis auf die Angebote anderer Regionen zu erzielen. Ausschlaggebend dürften jedoch die politik-ökonomischen Gründe sein. Die Regionaltheorien werden benutzt, um eine politisch begründete Umverteilung zu legitimieren. Das Land kann basierend auf seinem konstruktivistischen wirtschaftspolitischen Konzept und der Annahme des allokativen Marktversagens die Konzentration der Förderung in den schwachen Regionen begründen. Aus politik-ökonomischen Gründen vermag es diese Position jedoch nicht durchzuhalten, da der Druck aus den geringer geförderten Regionen sehr groß ist. Im Sinne eines nutzenmaximierenden Kalküls ist es dann durchaus rational, zur Sicherung der Regierungsmacht, die Verteilung der Gelder zu verschieben.

Die Analyse zeigt deutlich die Stärke einer ordnungspolitischen Regionalpolitik. Nur die Bindung an allgemeinverbindliche Regeln kann die rent-seeking und bargaining-Prozesse der Regionalpolitik unterbinden. Die Regionen könnten dann mit ihren wirtschaftspolitischen Konzeptionen und regionalpolitischen 
Programmen sowie ihren immobilen Faktoren in einen Wettbewerb um die mobilen Produktionsfaktoren treten.

\section{Wirtschaftspolitik und Wirtschaftsförderung des Landes Mecklen- burg-Vorpommern}

Die Analyse hat sich bisher auf die regionalpolitische Institutionen konzentriert, die hinsichtlich der Finanzierung sowie der inhaltlichen Ausgestaltung durch die EG, den Bund und das Land gemeinsam im Rahmen eines verflochtenen Systems definiert und implementiert werden. Ausgehend von dem Konzept einer ordnungspolitischen Regionalpolitik soll nunmehr geprüft werden, ob parallel zu diesem verflochtenen System eine eigenständige regelgebundene Regionalpolitik in Mecklenburg-Vorpommern möglich ist. Daher ist zunächst die Frage zu klären, ob es institutionelle Strukturen gibt, die Regeln für einen Wettbewerb der Regionen setzen und durchsetzen können. Es gilt somit zu untersuchen, ob und wenn ja, welche Rolle die GA bzw. die Beihilfenkontrolle der Gemeinschaft spielen. Da ein Wettbewerb zwischen regionalen Einheiten Handlungsfreiräume dieser Ebene voraussetzt, liegt der Schwerpunkt zunächst auf folgenden Fragen:

a. Hat das Land materielle regionalpolitische Handlungsfreiräume?

b. Wenn ja, sind die Handlungsfreiräume finanziell abgesichert?

Die materiellen Handlungsfreiräume werden im ersten Abschnitt untersucht. Anschließend wird am Beispiel Mecklenburg-Vorpommerns analysiert, ob und wenn ja, wie diese Freiräume finanziell abgesichert sind. In den folgenden $\mathrm{Ab}$ schnitten wird dann untersucht, wie das Land diese Freiräume mit eigenen Konzepten und Programmen ausfüllt. Hierbei stehen vier Fragen im Vordergrund:

a. Wie nutzt das Land die Freiräume?

b. Wo setzt es eigene Akzente?

c. Warum werden diese Akzente gesetzt?

d. Wie sind sie regionalpolitisch $\mathrm{zu}$ beurteilen?

\subsection{Handlungsfreiräume für eine eigenständige Wirtschaftspolitik des Landes}

\subsubsection{Das Land als Träger der regionalen Wirtschaftspolitik}

Die Länder sind Träger der regionalen Wirtschaftspolitik. Der Bund hat zwar im Rahmen der grundgesetzlichen Möglichkeiten die Gesetzgebungskompetenz für das Recht der Wirtschaft im wesentlichen an sich gezogen, jedoch bleibt hiervon zunächst die Zuständigkeit der Länder für die regionale Wirtschaftspolitik im allgemeinen und die regionale Wirtschaftsförderung im besonderen unangetastet. 
Gemäß Art. 91 a GG wirkt der Bund "... bei der Erfüllung von Aufgaben der Länder ..." mit. Die Verbesserung der regionalen Wirtschaftsstruktur ist somit eine originäre Länderaufgabe. Mitwirkung durch den Bund bedeutet allerdings auch Mitsprache und somit auch eine Einschränkung der Handlungsfreiräume der Länder. Dies gilt zunächst, wie oben gezeigt, für die GA selbst. Die Regelungen der GA entfalten jedoch auch Bindungswirkungen über ihren eigentlichen Geltungsbereich hinaus. Die GA will "... einheitliche Rahmenbedingungen für die Aktivitäten von Bund, Ländern und Gemeinden auf dem Gebiet der regionalen Wirtschaftsförderung ..."195 setzen. Programme der Länder, die neben der GA bestehen, "... dürfen die Ziele der Gemeinschaftsaufgabe nicht durchkreuzen"196. Für alle raumwirksamen Politikbereiche gilt, daß sie in enger Abstimmung zwischen Bund und Ländern erfolgen sollten, um ihre Effizienz zu erhöhen. Landeseigene Programme können somit die GA verstärken oder neben der GA andere Schwerpunkte setzen. Im zweiten Fall ist jedoch darauf zu achten, daß die Landesprogramme die GA nicht konterkarieren.

Die GA schafft für die Regionalpolitik einen bundeseinheitlichen Koordinierungsrahmen, der für Bund, Länder und Kommunen Gültigkeit hat. Länder und Kommunen sind gehalten, ihre eigene Wirtschaftspolitik und Wirtschaftsförderung an diesen Regeln auszurichten. Es gibt allerdings keinen Sanktionsmechanismus, der bei einem abweichenden Verhalten der Länder greifen könnte. Die Koordinationsfunktion des Systems, die u.a. einen Förderwettbewerb zwischen den Ländern unterbunden hat, ist nur so lange gewährleistet, wie die Interessen aller Beteiligten im Rahmen des Systems Berücksichtigung finden. Fraglich ist, ob die GA ihre Koordinierungsfunktion weiterhin erfüllen kann, wenn der Umfang der Fördergebiete durch die Gemeinschaft zunehmend eingeschränkt wird. Für Länder, die keine GA Fördergebiete haben (wie z.B. Baden-Württemberg) oder nur sehr geringe GA-Mittel erhalten, bekommen die Regeln der Beihilfenkontrolle der EG eine immer stärkere Bedeutung. Die Koordinierungsfunktion der GA wird somit mittelfristig durch die Strukturfonds und die zunehmend restriktivere Beihilfenkontrolle der EG ausgehöhlt.

Das Konzept der GA hat wenig mit einer ordnungspolitischen Regionalpolitik zu tun, da die GA nicht in der Lage ist, allgemeinverbindliche Regeln für die Wirtschaftsförderung gegenüber den Ländern durchzusetzen. Die GA versucht vielmehr, die Landesprogramme inhaltlich zu koordinieren und die Förderung in den Ländern nach bestimmten Prinzipien auszurichten. Dieser Ansatz läßt durchaus Raum für eigene regionalpolitische Aktivitäten der Länder.

Dreiundzwanzigster Rahmenplan, a.a.O., S. 8.

Dreiundzwanzigster Rahmenplan, a.a.O., S. 8. 
Um ein abschließendes Bild von den Handlungsspielräumen der Länder zu erhalten, müssen an dieser Stelle die Restriktionen der Beihilfenkontrolle der EG analysiert werden. Gemäß Art 92 ff EWG-Vertrag hat die Kommission das Recht, jede Beihilfe auf ihre Vereinbarkeit mit dem gemeinsamen Markt zu überprüfen. Ziel der Kontrolle ist es, Wettbewerbsverzerrungen durch staatliche Beihilfen sowie einen Förderwettbewerb zu verhindern. Der Beihilfenbegriff ist sehr weit gefaßt. Es können alle "Maßnahmen, welche die Belastungen vermindern, die ein bestimmtes Unternehmen oder eine bestimmte Gruppe von Unternehmen normalerweise nach innerstaatlichem Recht zu tragen hat" ${ }^{197}$ unter dem Begriff Beihilfe subsumiert werden.

Die Länder sind verpflichtet, ihre eigenen Programme über den Bund der Kommission zur Prüfung vorzulegen. ${ }^{198}$ Solange die Prüfung nicht abgeschlossen ist, dürfen die vorgelegten Programme nicht angewendet werden. Erst nach $\mathrm{Ab}$ schluß des Notifizierungsverfahrens, soweit die Kommission keine Bedenken gegen die vorgelegten Programme hat, darf das Land diese umsetzen.

Der Umfang und die Kriterien der Beihilfenkontrolle sind zunächst sehr allgemein im EWG-Vertrag geregelt. In bestimmten Fällen sind Beihilfen grundsätzlich mit dem gemeinsamen Markt vereinbar. ${ }^{199}$ Dies gilt für Beihilfen sozialer Art an einzelne Verbraucher und Hilfen zur Beseitigung von Schäden, die durch Naturkatastrophen oder andere außergewöhnliche Ereignisse verursacht worden sind. Ferner fielen bzw. fallen auch Beihilfen für Gebiete, die von der Teilung Deutschlands betroffen sind, unter diese Rubrik. ${ }^{200}$ Andere Beihilfen können mit dem gemeinsamen Markt vereinbar sein. ${ }^{201}$ Dies gilt unter folgenden Bedingungen.

a. Beihilfen für Gebiete, in denen die Lebenshaltung niedrig ist oder erhebliche Unterbeschäftigung herrscht

b. Beihilfen zur Behebung von Störungen im Wirtschaftsleben bzw. für Vorhaben, die von europäischem Interesse sind

197 Entscheidung des EUGH zitiert nach von der Groeben et alia: "Kommentar zum EWGVertrag", 1983. Eine ausfuhrliche Darstellung zum Beihilfenbegriff findet sich bei Grabitz, Hilf: "Kommentar zur Europăischen Union", C.H. Beck, München 1992.

Der Bund wird durch den EWGV zur Notifizierung der Beihilfen verpflichtet. Die Lănder müssen im Rahmen der Bundestreue den Bund von eigenen Beihilfen rechtzeitige in Kenntnis setzen.

Vgl. Art 92 (2) EWG-Vertrag.

Die Zonenrandforderung sowie der Berlin-Forderung wurden hierunter subsumiert.

Vgl. Art 92 (3) EWG-Vertrag. 
c. Beihilfen zur Förderung eines Wirtschaftsgebietes oder Wirtschaftszweiges

d. Beihilfen zur Förderung der Kultur ${ }^{202}$

e. sonstige Arten von Beihilfen, die der Rat auf Vorschlag der Kommission beschließt

Gemäß Art. 92 (3) kann somit eine breite Palette von Beihilfen mit dem gemeinsamen Markt vereinbar sein. Die Prüfung der Vereinbarkeit obliegt grundsätzlich der Kommission. Der Rat kann auf Vorschlag der Kommission "... alle zweckdienlichen Durchführungsverordnungen zu den Artikeln 92 und 93 erlassen."203 Diese Vorschrift hat jedoch bisher keine Bedeutung erlangt. Die jeweils gültigen Prüfkriterien werden somit durch die Kommission bestimmt. Die für die Beihilfenkontrolle relevanten Entscheidungen, Verordnungen und Richtlinien haben sich seit $1972^{204} \mathrm{zu}$ einem sehr komplizierten Regelwerk entwickelt, das ständigen Erweiterungen und Veränderungen unterworfen ist. ${ }^{205}$ Die Prüfung bzw. Koordinierung der Beihilfen erstreckt sich hauptsächlich auf fünf Aspekte: ${ }^{206}$

a. Intensität der Förderung. Hierzu hat die Kommission regional differenzierte Intensitätshöchstgrenzen erlassen

b. Transparenz der Beihilfen

c. Regionale Spezifität der Beihilfen

d. Berücksichtigung sektoraler Auswirkungen

e. Einführung eines Überwachungssystems

Die primäre Zielsetzung der Beihilfenkontrolle ist die Sicherung eines fairen Wettbewerbs im gemeinsamen Markt durch die Unterbindung eines ruinösen Förderwettbewerbs. Staatliche Beihilfen verzerren den Wettbewerb und sind daher grundsätzlich mit dem gemeinsamen Markt unvereinbar. Allerdings ist ein fairer Wettbewerb nach Ansicht der EG nur dann möglich, wenn die Unternehmen bzw. Regionen annähernd gleiche Ausgangsbedingungen haben. Starke

202 Dieser Fördertatbestand wurde durch die Maastrichter Verträge ergänzt.

203 Art 94 EWG-Vertrag.

2041972 erließ die Kommission die ersten Koordinierungsgrundsătze, die einem Förderwettbewerb verhindern sollten. Diese Grundsätze wurden in Zusammenarbeit mit den Mitgliedstaaten bis 1978 uberarbeitet und 1979 im Amtsblatt veröfentlicht (Abl. $1979 \mathrm{Nr}$. C31 vom 03.02.1997, S. 9).

205 Die derzeit fur die GA und die Länder relevanten Vorgaben sind im 23. Rahmenplan zusammengestellt. Vergl. hierzu "Dreiundzwanzigster Rahmenplan", a.a.O., S. 18/19.

Vgl. hierzu die Koordnierungsgrundsătze der Kommission von 1979, a.a.O., S 9 ff. 
Disparitäten, z.B. im Bereich der Infrastrukturausstattung oder der Produktivität wirken ebenso wettbewerbsverzerrend wie staatliche Beihilfen.

Aus diesem Grund bekommen die Wettbewerbsregeln eine regionalpolitische Komponente. Staatliche Beihilfen, die Disparitäten abbauen, sind nicht nur mit dem gemeinsamen Markt vereinbar, sie sind vielmehr eine Voraussetzung für fairen Wettbewerb, da sie zu einer Angleichung der Rahmenbedingungen führen. Um diesen Ansatz umsetzen zu können, muß die Kommission eine Vorstellung von den regionalen Strukturproblemen haben, die die Wettbewerbsfähigkeit einer Region beeinträchtigen. Das heißt, daß sie über folgende Informationen verfügen $\mathrm{muß:}$

a. Die Kommission muß die Faktoren kennen, die die Wettbewerbsfähigkeit einer Region im innergemeinschaftlichen Handel beeinträchtigen und somit zu einer Verzerrung des Wettbewerbs führen

b. Sie muß ferner in der Lage sein, über diese Faktoren die Benachteiligung zu quantifizieren. Beihilfen sind nur insoweit mit dem gemeinsamen Markt vereinbar, wie sie Wettbewerbsverzerrungen ausgleichen. Daher kann der zulässige Umfang und die Höhe einer Beihilfe nur bestimmt werden, wenn das Ausmaß der Benachteiligung über die Faktoren quantifizierbar ist

Um diese Aufgabe erfüllen zu können, müßte die Kommission Kenntnis über die regionalwirtschaftlichen Funktionszusammenhänge haben. Nur dann kann sie die Faktoren identifizieren, die die Wettbewerbsfähigkeit beeinträchtigen und nur dann kann sie beurteilen, ob die vorgeschlagenen Programme die Defizite beheben können. Die Beihilfenkontrolle als passive Regional- und Strukturpolitik steckt daher in dem gleichen Dilemma wie die aktive Regional- und Strukturpolitik.

a. Eine so verstandene Beihilfenkontrolle basiert auf einem konstruktivistischen wirtschaftspolitischen Konzept. Um eine rationale Kontrolle zu ermöglichen, muß sie sich an eigenen regional- und strukturpolitischen Zielen orientieren. Die Wettbewerbskontrolle ist dann nicht mehr die Durchsetzung allgemeinverbindlicher Regeln, sondern sie steht im Dienst des regional- und strukturpolitischen Leitbildes der Kommission. Dies führt zur Mißachtung der regionalen Präferenzen und somit zu erheblichen Wohlfahrtsverlusten.

b. Die regionalwirtschaftlichen Theorien sind nicht in der Lage, die zur Umsetzung dieser Strategie notwendigen Antworten zu geben. Weder sind die Faktoren, die den Wettbewerb verzerren, eindeutig zu identifizieren noch sind diese ausreichend quantifizierbar. Die zentralisierte Umsetzung dieses Kontrollkonzeptes induziert ferner einen nicht zu bewältigenden Informationsbeschaffungs- und Informationsverarbeitungsbedarf. 
c. Wie bei der Programmplanung der GA und des EFRE tendiert dieses System zu einer Dominanz der politischen Rationalität. Die Beihilfenkontrolle ist nicht Durchsetzung allgemeinverbindlicher Regelungen, sondern ist de facto die Einzelfallprüfung von Programmen und Maßnahmen. Da die Regeln nicht abschließend definiert sind, ist viel Raum für politische bargaining-Prozesse. Der politische Druck auf die Kommission bei derartigen Entscheidungen ist erheblich und führt nicht selten zur Genehmigung der gewünschten Beihilfen ${ }^{207}$

Diese Struktur läßt keinen Raum für eine ordnungspolitische Regionalpolitik, ein regelgebundener Wettbewerb der regionalen Einheit ist nicht möglich. Der Handlungsspielraum der Länder für eigene regional- und strukturpolitische Programme, die sich an den regionalen Präferenzen orientieren, wird durch die Beihilfenkontrolle der Kommission erheblich eingeschränkt.

Es gibt umfangreiche inhaltliche Restriktionen, die ständiger Veränderung bzw. Erweiterung unterliegen. In diesem Zusammenhang sei nochmals auf die bereits erwähnte Ausweitung der Beihilfenkontrolle auf die Förderung von Infrastrukturinvestitionen hingewiesen. Der sehr weite und unbestimmte Beihilfenbegriff ermöglicht es der Kommission, die Kontrolle zunehmend auszuweiten.

Die Länder müssen die inhaltlichen Vorgaben der Kommission bei der Aufstellung ihrer Programme berücksichtigen, damit die Notifizierung erfolgreich abgeschlossen werden kann. Dies führt zwangsläufig zu einer stärkeren Orientierung an den europäischen und einer Vernachlässigung der regionalen Präferenzen.

Ferner wird der Handlungsspielraum der Länder durch den erheblichen Verwaltungs- und Zeitaufwand eingeschränkt, den eine Notifizierung mit sich bringt. $\mathrm{Da}$ das Land seine Beihilfen nicht direkt bei der Kommission notifizieren kann, muß zunächst der Bund eingeschaltet werden. Bereits auf dieser Ebene erfolgt eine Beratung bzw. Beeinflussung des Landes, um die Erfolgsaussichten einer Notifizierung zu erhöhen. Ob eine Länderbeihilfe genehmigt wird, hängt sicherlich auch von der politischen Unterstützung des Bundes ab. Auf dieser Ebene fließen dann neben den Vorgaben der Kommission die Präferenzen des Bundes in den Notifizierungsprozeß ein, was zu einer weiteren Verwischung der originären Länderpräferenzen führen kann.

Dieser Notifizierungsprozeß kann sich sehr lange hinziehen. Nach der Rechtsprechung des EUGH muß sich die Kommission spätestens nach acht Wochen grundsätzlich zu einer ihr zur Genehmigung vorgelegten Beihilfe äußern. Wenn 
keine Äußerung erfolgt, gilt die Beihilfe als bestehend. ${ }^{208}$ Hat die Kommission allerdings Einwendungen, wird das in Art. 93 (2) EWGV festgelegte Verfahren eingeleitet. Dies führt dann im Ergebnis zu einer verbindlichen Entscheidung, die dem Land innerhalb einer bestimmten Frist die Aufhebung bzw. Umgestaltung der Beihilfe auferlegt. Am Beispiel des Investitionssicherungsprogramms des Landes Mecklenburg-Vorpommern lassen sich die Verzögerungen gut verdeutlichen. Dieses Programm wurde im Laufe des Jahres 1993 aufgelegt ${ }^{209}$. Mit ihm sollten noch nicht abgeschlossene Investitionsprojekte von KMU, die jedoch an Finanzierungsproblemen zu scheitern drohten, mit Zuschüssen gefördert werden. ${ }^{210}$ Die ursprüngliche Notifizierung wurde im Juli 1994 aufgrund einer Vielzahl von Fragen der Kommission zurückgezogen und gleichzeitig in textlich geänderter Form neu eingereicht. Die Richtlinie des Investitionssicherungsprogramms trat dann zum 01.09.1994 in Kraft und lief Ende 1994 aus. Das Land wollte mit diesem Programm auf eine akute und zeitlich befristete Notlage reagieren. Wie dies Beispiel sehr deutlich zeigt, führte die Verzögerung des Notifizierungsverfahrens diesen Versuch ad absurdum. Das Land kann zwar Maßnahmen und Programme ankündigen, muß deren Umsetzung jedoch aussetzen, solange die Notifizierung nicht erfolgreich abgeschlossen ist. Damit ist eine sinnvolle Reaktion auf konkrete Notlagen nicht möglich und die Effektivität der eingesetzten Mittel wird erheblich gemindert.

Auch die Beihilfenkontrolle orientiert sich nicht an einem ordnungspolitischen Konzept der Regionalpolitik, sie setzt keine allgemeinverbindlichen Regeln, sondern steht vielmehr im Dienst der regional- und strukturpolitischen Ziele der Kommission. Die Länder haben in dieser Struktur grundsätzlich die Möglichkeit, eigene regionalpolitische Programme aufzulegen, müssen diese jedoch durch die Kommission genehmigen lassen. Dieser Genehmigungsvorbehalt limitiert die Freiräume der Länder sehr stark und reduziert die Optionen auf die Programme, die nicht im Widerspruch zu den grundsätzlichen Zielen der Kommission stehen.

\subsubsection{Finanzielle Handlungsspielräume des Landes Mecklenburg- Vorpommern}

Neben den institutionellen Bedingungen, die die Politikfähigkeit der Länder bestimmen, ist die finanzielle Absicherung von Handlungsfreiräumen von be-

Es ist bezeichnend, daß der EUGH derartige Verfahrensfragen zu regeln hat. Der Rat war jedoch nicht in der Lage, einen gleichlautenden Vorschlag der Kommission zu beschließen. Vgl. hierzu ausfuhrlich, "Kommentar zum EWG-Vertrag", a.a.O., S. 1637/1638.

Diesen Zeitraum nennt jedenfalls der Jahreswirtschaftsbericht 1995 von MecklenburgVorpommern. Vgl. Ministerium furr Wirtschaft: "Jahreswirtschaftsbericht 1995 Mecklenburg-Vorpommern", 31.07.1995, S. 80.

Vgl. Ministerium fur Wirtschaft: "Jahreswirtschaftsbericht 1995 Mecklenburg-

Vorpommern", a.a.O., S. 80. 
sonderem Interesse. Potentielle Handlungsfreiräume sind nur insoweit ausfüllbar, wie die hierfür notwendigen finanziellen Mittel zur Verfügung stehen. Tabelle 13 gibt Aufschluß über die finanziellen Handlungsspielräume des Landes. In der ersten Zeile ist die Summe der regional- und strukturpolitischen Ausgaben des Landes Mecklenburg-Vorpommern gemäß den Haushaltsansätzen der jeweiligen Jahre dargestellt.

Tabelle 13

\begin{tabular}{|c|c|c|c|c|c|c|}
\hline \multicolumn{7}{|c|}{$\begin{array}{l}\text { Bedeutung der Wirtschaftsförderung des Landes Mecklenburg-Vorpommern } \\
\text { (Angaben in Prozent bzw. } 1000 \text { DM) }\end{array}$} \\
\hline & 1991 & 1992 & 1993 & 1994 & 1995 & 1996 \\
\hline \multicolumn{7}{|l|}{ Ausgaben } \\
\hline Landesprogr. & 77.488 & 75.632 & 133.773 & 145.120 & 200.829 & 172.998 \\
\hline $\begin{array}{l}\text { GA, AO, } \\
\text { EFRE }\end{array}$ & 625.300 & 850.600 & 1.056 .100 & 1.122 .353 & 875.440 & 1.004 .018 \\
\hline $\begin{array}{l}\text { Gemeinschafts } \\
\text {-initiativen } \\
\text { (GI) }\end{array}$ & 0 & 0 & 0 & 0 & 51.932 & 54.441 \\
\hline Gesamtförd. & 702.788 & 926.232 & 1.189 .873 & 1.267 .473 & 1.128 .201 & 1.231 .457 \\
\hline \multicolumn{7}{|l|}{ Einnahmen } \\
\hline Zuweis. GA & 178.500 & 262.150 & 470.050 & 443.644 & 319.220 & 377.700 \\
\hline Zuweis. AO & 75.000 & 104.000 & 0 & 0 & 0 & 0 \\
\hline Zuweis. EFRE & 118.300 & 118.300 & 116.000 & 235.065 & 237.000 & 248.618 \\
\hline Zuweis. GI & 0 & 0 & 0 & 0 & 41.034 & 43.020 \\
\hline Summe & 371.800 & 484.450 & 586.050 & 678.709 & 597.254 & 669.338 \\
\hline $\begin{array}{l}\text { Landesanteil } \\
\text { zur Kofinanz. }\end{array}$ & 253.500 & 366.150 & 470.050 & 443.644 & 330.118 & 389.121 \\
\hline $\begin{array}{l}\text { Anteil der } \\
\text { Landes- } \\
\text { programme }\end{array}$ & $11 \%$ & $8 \%$ & $11 \%$ & $11 \%$ & $18 \%$ & $14 \%$ \\
\hline $\begin{array}{l}\text { Anteil GA, } \\
\text { AO, EFRE, GI }\end{array}$ & $89 \%$ & $92 \%$ & $89 \%$ & $89 \%$ & $82 \%$ & $86 \%$ \\
\hline $\begin{array}{l}\text { Anteil GA, } \\
\text { AO, EFRE, GI } \\
\text { am Gesamt- } \\
\text { haushalt }\end{array}$ & $34 \%$ & $50 \%$ & $56 \%$ & $59 \%$ & $53 \%$ & $48 \%$ \\
\hline
\end{tabular}


Die in der Verfassung theoretisch verankerte Aufgabe der Länder als Träger der Wirtschaftsförderung wird de facto vom Bund bzw. der EG wahrgenommen. Die landeseigenen Programme umfassen im Jahr 1991 ca. 77 Mill. DM, steigen bis zum Jahr 1995 auf 200 Mill. DM an und liegen 1996 bei 173 Mill. DM. In der dritten Zeile sind die Ausgaben im Rahmen von Gemeinschaftsinitiativen dargestellt. Gemeinschaftsinitiativen sind erst seit 1994 mit der offiziellen Eingliederung der neuen Länder in die Regelungen der Strukturfonds und der Zuordnung zum Ziel-1-Gebiet möglich. Ein bestimmter Prozentsatz der EFRE Mittel ist für derartige Initiativen reserviert, die entweder auf Vorschlag des Landes oder der Kommission eingerichtet werden. Die Programme werden unter Ausschluß des Bundes gemeinsam vom Land und der EG getragen. Im Jahr 1995 wurden hierfür ca. 52 Mill. DM ausgegeben, für das Jahr 1996 sind 54 Mill. DM vorgesehen. Die meisten Mittel stehen allerdings im Rahmen der GA, des AO sowie des EFRE zur Verfügung. Im Jahr 1991 waren dies ca. 625. Mill. DM, 1994 stand mit ca. 1,1 Mrd. DM die höchste Summe zur Verfügung. Wie bereits oben erwähnt, muß das Land die Mittel, die vom Bund bzw. von der EG zugeführt werden, kofinanzieren. Dieser Landesanteil an der Förderung beträgt 1991 253 Mill. DM und steigt bis 1993 auf 470 Mill. DM an. Parallel zu den Zuweisungen des Bundes nimmt der Landesanteil 1994 und 1995 ab. Für das Jahr 1996 sind 389 Mill. DM zur Kofinanzierung in den Haushalt eingestellt. Ein erheblicher Teil der Landesmittel ist somit für die Kofinanzierung von GA, AO und EFRE gebunden.

Der Anteil der Ausgaben im Rahmen GA, AO, EFRE an den Gesamtausgaben für Wirtschaftsförderung schwankt in den Jahren 1991-96 zwischen 80\% und $90 \%$. Der Anteil der Landesprogramme liegt in den ersten vier Jahren zwischen $8 \%$ und $11 \%$ und steigt erst 1995 auf $18 \%$ an, fällt allerdings 1996 auf $14 \%$ zurück. Auch bezogen auf den Gesamthaushalt des Wirtschaftsministers haben die Ausgaben für GA, AO und EFRE ein erdrückendes Gewicht. Der Anteil wächst kontinuierlich von 34,3\% im Jahr 1991 auf 59\% 1995, fällt 1996 jedoch auf 48\% ab.

Diese Zahlen machen die geringen finanziellen Handlungsspielräume des Landes sehr deutlich. Die Wirtschaftsförderung wird von der GA und dem EFRE dominiert. Mit der zu erwartenden Reduzierung der Zuweisungen des Bundes im Rahmen der GA wird der EFRE bis zum Jahr 1999 eine stärkere Bedeutung bekommen. Dieser hohe Grad der finanziellen Abhängigkeit des Landes von Zweckzuweisungen in einem Kernbereich der Landespolitik wird auch bei einer Analyse des Gesamthaushaltes deutlich. Betrachtet man die Einnahmen des Wirtschaftsministeriums aus zweckgebundenen Zuweisungen, so ergibt sich folgendes Bild (Tabelle 14): Der Anteil der zweckgebundenen Zuweisungen liegt in allen Jahren über 30\%. Im Jahr 1996 hat er einen Spitzenwert von 50\%, 1994 liegt er bei 45\%. Diese Zahlen machen die starke Abhängigkeit der neuen 
Länder von Bundeszuweisungen deutlich. Aus eigenen Einnahmequellen sind die Aufgaben des Landes im Bereich des Wirtschaftsministeriums nicht zu finanzieren. Auf der anderen Seite spiegeln sie aber auch die starke Einflußnahme des Bundes wider, der im Bereich des Wirtschaftsministeriums nur zweckgebundene Zuschüsse zur Verfügung stellt. Aus Sicht der Theorie des fiskalischen Föderalismus will es nicht einleuchten, warum der Bund Zweckzuweisungen für kommunale Straßen bzw. für den Ausbau des ÖPNV zur Verfügung stellt.

Tabelle 14

Bedeutung von Zweckzuweisungen im Etat des Wirtschaftsministeriums (Angaben in $1000 \mathrm{DM}$ )

\begin{tabular}{|c|c|c|c|c|c|c|}
\hline & 1991 & 1992 & 1993 & 1994 & 1995 & 1996 \\
\hline \multicolumn{7}{|l|}{ Kapitel 0602} \\
\hline $\begin{array}{l}\text { Bund: kom- } \\
\text { munale Stra- } \\
\text { Ben }\end{array}$ & 65.500 & 65.534 & 65.500 & 81.677 & 60.954 & 61.258 \\
\hline Bund: ÖPNV & 26.910 & 45.420 & 65.000 & 81.677 & 60.954 & 61.257 \\
\hline $\begin{array}{l}\text { Bund: Fern- } \\
\text { wärme }\end{array}$ & 0 & 17.300 & 17.300 & 17.300 & 17.300 & 0 \\
\hline $\begin{array}{l}\text { Regionaler } \\
\text { SPNV/ÖPNV } \\
\end{array}$ & 0 & 0 & 0 & 0 & 0 & 293.000 \\
\hline $\begin{array}{l}\text { AO: kommu- } \\
\text { nale Straßen }\end{array}$ & 66.000 & 158.900 & 0 & 0 & 0 & 0 \\
\hline AO: ÖPNV & 20.350 & 18.500 & 0 & 0 & 0 & 0 \\
\hline $\begin{array}{l}\text { EU: Gemein.- } \\
\text { Initiative }\end{array}$ & 0 & 0 & 0 & 0 & 42.694 & 43.020 \\
\hline \multicolumn{7}{|l|}{ Kapitel 0603} \\
\hline $\begin{array}{l}\text { EFRE: Techn. } \\
\text { Hilfe }\end{array}$ & 2.870 & 2.884 & 3.000 & 2.374 & 2.398 & 2.550 \\
\hline EFRE: GA & 118.300 & 118.300 & 116.000 & 235.065 & 237.000 & 248.618 \\
\hline $\begin{array}{l}\text { Bund: GA } \\
\text { Infra }\end{array}$ & 89.250 & 131.075 & 235.025 & 221.822 & 127.688 & 151.080 \\
\hline $\begin{array}{l}\text { Bund: Ga } \\
\text { Gew. }\end{array}$ & 89.250 & 131.075 & 235.025 & 221.822 & 191.532 & 226.620 \\
\hline $\mathrm{AO}$ & 75.000 & 104.000 & 0 & 0 & 0 & 0 \\
\hline Summe: & 553.430 & 792.988 & 736.850 & 861.736 & 740.520 & 1.087 .403 \\
\hline Gesamthaush. & 1.823 .278 & 1.693 .148 & 1.884 .404 & 1.897 .473 & 1.754 .554 & 2.184 .923 \\
\hline $\begin{array}{l}\text { Anteil der } \\
\text { Zuweisungen }\end{array}$ & $30 \%$ & $47 \%$ & $39 \%$ & $45 \%$ & $42 \%$ & $50 \%$ \\
\hline
\end{tabular}


Die finanziellen Handlungsspielräume des Landes zur Durchführung eigener wirtschaftspolitischer Maßnahmen sind auf ein Minimum reduziert. Das Land hat insbesondere im Bereich der Wirtschaftsförderung weder institutionell noch finanziell die notwendigen Handlungsfreiräume, um eigene Konzepte und Programme effektiv durchzuführen.Die landeseigenen Programme umfassen im Jahr 1991 ca. 77 Mill. DM, steigen bis zum Jahr 1995 auf 200 Mill. DM an und liegen 1996 bei 173 Mill. DM.

Die Dominanz der bundespolitischen Maßnahmen wird noch deutlicher, wenn man die steuerlichen Fördermaßnahmen in die Betrachtung einbezieht (Tabelle $15)^{211}$. Allein die in den neuen Ländern ausgezahlte Investitionszulage ist annähernd genauso hoch wie die Investitionszuschüsse der GA. Diese Aussage gilt allerdings nur für die Bruttoförderung. Berücksichtigt man die Tatsache, daß die Investitionszuschüsse der GA zu versteuern sind, so wird deutlich, daß die Nettoförderung der GA erheblich unter der nicht zu versteuernden Investitionszulage liegt. ${ }^{212}$ Von Bedeutung sind ferner die Steuermindereinnahmen durch die Sonderabschreibungen, die sich für Betriebe 1994 auf 2,6 Mrd. DM summieren und somit auch über der Nettoförderung der GA 1994 liegen.

Bei den Steuermindereinnahmen ist zu berücksichtigen, daß diese nicht den Bund alleine treffen, sondern zum Teil von den Ländern mitzutragen sind und den finanziellen Spielraum der Länder zusätzlich einengen. Die auf Bundesebene beschlossenen Fördermaßnahmen "Investitionszulage" und "Sonderabschreibung" haben ein höheres Volumen als die Brutto- bzw. Nettoförderung privater Investitionen der GA.

Im Lichte dieser Zahlen wird die geringe Dimension der landeseigenen Fördermaßnahmen deutlich. Die Regionalförderung in den neuen Ländern wird eindeutig vom Bund dominiert. Inhalt und Umfang der Fördermaßnahmen werden maßgeblich vom Bund bestimmt, eine Berücksichtigung regionaler Präferenzen und Besonderheiten findet nicht statt. Auch das Prinzip der fiskalischen Äquivalenz wird bei den steuerlichen Fördermaßnahmen nicht berücksichtigt. Dies ist allerdings im Rahmen der bestehenden Finanzverfassung mit ihrer Zuordnung von Steuerquellen zu den verschiedenen Ebenen des föderalen Systems auch nicht möglich.

Quelle: Ifo-Studie „Die Effizienz der finanzpolitischen Fördermaßnahmen in den neuen Bundesländern", a.a.O., S. 113.

Der Subventionswert einer Zulage von $12 \%$ ist höher als der Subventionswert eines Investitonszuschußes von $15 \%$. Der Zuschuß hat in Abhăngigkeit von der Art der Investition und des gewăhlten Diskontierungssatzes einen Subventionswert von maximal 9,78\%, die Zulage hat hingegen selbst im ungünstigsten Fall noch einen Subventionswert von $10,86 \%$. Ausfuhrliche Berechnungen hierzu finden sich in: ifo Studie "Die Effizienz der finanzpolitischen Fördermaßnahmen in den neuen Bundeslăndern", a.a.O., S. 25-36. 
Tabelle 15

\begin{tabular}{|c|c|c|c|c|}
\hline \multicolumn{5}{|c|}{$\begin{array}{c}\text { Fiskalische Kosten der Förderung privater Investitionen in den } \\
\text { Neuen Bundesländern }{ }^{1)} \text { (Mrd. DM) }\end{array}$} \\
\hline & 1991 & 1992 & 1993 & 1994 \\
\hline $\begin{array}{l}\text { Investitionszulage } \\
\text { dar.: Auszahlungen in den } \\
\text { NBL }\end{array}$ & $1,04^{2)}$ & $\begin{array}{l}4,30 \\
3,41\end{array}$ & $\begin{array}{l}4,60 \\
4,20\end{array}$ & $\begin{array}{l}5,50 \\
4,82\end{array}$ \\
\hline $\begin{array}{l}\text { Sonderabschreibung } \\
\text { a. Betriebe } \\
\text { b. privater Wohnungsbau }\end{array}$ & $\begin{array}{l}1,45 \\
0,14\end{array}$ & $\begin{array}{l}1,51 \\
0,39\end{array}$ & $\begin{array}{l}2,40 \\
0,49\end{array}$ & $\begin{array}{l}2,60 \\
0,65\end{array}$ \\
\hline $\begin{array}{l}\text { Gewerbesteuer } \\
\text { a. Gewerbeertragsteuer } \\
\text { b. Gewerbekapitalsteuer }\end{array}$ & $\begin{array}{l}0,10 \\
0,15\end{array}$ & $\begin{array}{l}0,10 \\
0,21\end{array}$ & $\overline{-}$ & $\overline{0}, \overline{13}$ \\
\hline Vermögenssteuer" & 0,20 & 0,30 & 0,35 & 0,44 \\
\hline $\begin{array}{l}\text { Summe der } \\
\text { Steuermindereinnahmen }\end{array}$ & 3,08 & 6,81 & 7,94 & 9,32 \\
\hline $\begin{array}{l}\text { InvestitionszuschuB (GA) } \\
\left.\text { a. brutto }{ }^{6}\right) \\
\text { b. netto }\end{array}$ & $\begin{array}{l}3,14 \\
1,32\end{array}$ & $\begin{array}{l}4,27 \\
1,79\end{array}$ & $\begin{array}{l}5,61 \\
2,36\end{array}$ & $\begin{array}{c}4,06^{8)} \\
1,71\end{array}$ \\
\hline $\begin{array}{l}\text { Fiskalische Kosten der privaten } \\
\text { Investitionsförderung insge- } \\
\text { samt }\end{array}$ & 4,40 & 8,60 & 10,30 & 11,03 \\
\hline \multicolumn{5}{|c|}{$\begin{array}{l}\text { 1)Rechnungsjahre, offizielle Schätzungen. 2) Für } 1991 \text { ohne Auszahlungen in den alten } \\
\text { Bundesländern. 3) Einschließlich Förderung über höhere Sonderausgaben (maximal } \\
40.000 \text { DM). 4) Staffelung der Meßzahl nach dem Gewerbeertrag (ab 1993 bundesein- } \\
\text { heitlich). 5) Steuerausfälle durch Nichterhebung. 6) } 1991 \text { (einschließlich 4. Quartal } \\
\text { 1990) und 1992 Mittelabflüsse; } 1993 \text { und } 1994 \text { Finanzpläne der Länder. 7) Auszahlungen } \\
\text { abzüglich Steuerrückflüsse (Annahme: Grenzgewinnsteuersatz 58\%).8) Plandaten vor } \\
\text { Aufstellung des Bundeshaushaltes 1994. }\end{array}$} \\
\hline \multicolumn{5}{|c|}{$\begin{array}{l}\text { Quelle: Berechnungen des ifo, BMF, Dreizehnter Subventionsbericht, 22. GA- } \\
\text { Rahmenplan, BMWi-Außenstelle Berlin, AK-Steuerschätzung. }\end{array}$} \\
\hline
\end{tabular}

Eine ordnungspolitische Regionalpolitik ist im Rahmen dieser Verteilung der Finanzierungskompetenzen nur sehr eingeschränkt möglich. Ein Wettbewerb der Regionen wird verhindert, es dominieren die einheitlichen, zentralstaatlichen Lösungen. Programminnovationen der Länder werden durch die institutionellen Restriktionen erschwert und spielen auf Grund des sehr geringen finanziellen Umfanges nur eine untergeordnete Rolle. 


\subsection{Wirtschaftspolitik und Wirtschaftsförderung des Landes Mecklen- burg-Vorpommern}

Obwohl, oder gerade weil, die Handlungs- und Finanzierungskompetenzen des Landes erheblich eingeschränkt sind, haben die landeseigenen Konzepte und Programme eine hohe politische Bedeutung. Das Land versucht im Rahmen seiner Aktivitäten, bewußt eigene Schwerpunkte zu setzen und orientiert sich sehr stark an regional bedeutsamen wirtschaftspolitischen Problemfeldern. Die folgende Darstellung und Analyse der regionalpolitischen Programme des Landes orientiert sich an vier Fragen:

a. Wie nutzt das Land die geringen Freiräume?

b. Wo setzt es eigene Akzente?

c. Warum werden diese Akzente gesetzt?

d. Wie sind sie regionalpolitisch zu beurteilen?

Zunächst wird ein Überblick über die Handlungsfelder der Landeswirtschaftspolitik gegeben. Anschließend wird die Wirtschaftsförderung getrennt nach Mitteln und Instrumenten analysiert.

\subsubsection{Handlungsfelder der Wirtschaftspolitik und der Wirt- schaftsförderung des Landes Mecklenburg-Vorpommern}

Wirtschaftspolitik und Wirtschaftsförderung des Landes MecklenburgVorpommern lassen sich unter vier Handlungsfeldern zusammenfassen. Diese vier Handlungsfelder sind die Mittelstandspolitik, die Technologiepolitik, die Politik für den ländlichen Raum sowie die Tourismuspolitik.

Im Zentrum der Wirtschaftspolitik steht der Mittelstand, der für die wirtschaftliche Entwicklung des Landes im Rahmen des Transformationsprozesses von entscheidender Bedeutung ist. ${ }^{213}$ Die Förderung des Mittelstandes wurde bereits in der Koalitionsvereinbarung festgeschrieben ${ }^{214}$ und Ende 1993 in einem Gesetz verankert $^{215}$. Das Gesetz legt Fördermaßnahmen zur Steigerung der Leistungsfähigkeit sowie zur Verbesserung der Kapitalausstattung von KMU fest. Ferner

213 Diese Einschätzung des Wirtschaftsministers verdeutlicht folgendes Zitat sehr anschaulich: "Bei der Anpassung unseres Landes an eine auf Wettbewerb und Privateigentum ausgerichteten freiheitlichen Wirtschaftsordnung hat der Mittelstand eine besondere Bedeutung.", Wirtschaftsminister des Landes Mecklenburg-Vorpommern: "Der Mittelstand in Mecklenburg-Vorpommern", Schwerin 1994, S. 6.

Vgl. "Koalitionsvereinbarung zwischen dem Landesverband der CDU und dem Landesverband der FDP Mecklenburg-Vorpommern", Schwerin, Oktober 1990.

215 Gesetz zur wirtschaftlichen Flankierung des Mittelstandes in Mecklenburg-Vorpommern - Mittelstandsforderungsgesetz, vom 14.12.1993. 
verpflichtet es die Landesregierung, mindestens einmal in der Legislaturperiode einen Mittelstandsbericht vorzulegen.

Die drei anderen Handlungsfelder haben alle eine mehr oder weniger starke Ausrichtung auf den Mittelstand und weisen auch untereinander Überschneidungen auf. Der Tourismus ist für das Land ein wichtiger Wirtschaftszweig. Er soll Beschäftigung und Einkommen sichern, attraktive Erlebnis- und Erholungsmöglichkeiten bieten, eine nachhaltige Sicherung der Natur gewährleisten und die Bevölkerung nicht unangemessen belasten. ${ }^{216} \mathrm{Da}$ die Tourismuswirtschaft überwiegend mittelständisch geprägt ist, unterstützt die Tourismusförderung die Ziele der Mittelstandspolitik. Für ländliche Gebiete in MecklenburgVorpommern ist der Tourismus oftmals die bedeutendste Quelle für Einkommen und Beschäftigung. Daher spielt die Tourismusförderung eine große Rolle im Rahmen der Politik für den ländlichen Raum. ${ }^{217}$ Der ländliche Raum in MVP ist von der Anpassungskrise besonders hart betroffen. Im Zuge der Umstrukturierung der Landwirtschaft wurden viele Arbeitskräfte freigesetzt, für die in anderen Bereichen keine neuen Arbeitsplätze geschaffen werden konnten. Die ohnehin schon geringe Bevölkerungsdichte wird daher durch Abwanderungen weiter reduziert. Diese Entleerungstendenzen verstärken die wirtschaftlichen Probleme, da eine Ansiedlung von Dienstleistungsunternehmen oder Gewerbe unter solchen Bedingungen erheblich erschwert wird. Die Landesregierung von Mecklenburg-Vorpommern will daher im ländlichen Raum unter anderem die Landwirtschaft stabilisieren, den Mittelstand, den Fremdenverkehr und Technologiestandorte fördern. ${ }^{218}$ Politik für den ländlichen Raum hat somit etwas mit Tourismuspolitik, Mittelstandspolitik und Technologiepolitik zu tun.

Das Land möchte sich als moderner, technologie- und innovationsfreundlicher Wirtschaftsstandort profilieren. Forschung und Entwicklung ist nicht nur für die Erhöhung der Produktivität von Bedeutung, sondern stellt eine "... unabdingbare Voraussetzung zum Schutz von Umwelt und Klima, sowie zur Erweiterung sozialer Chancen, d.h. perspektivisch sicherer Arbeitsplätze ..."219 dar. Die Technologieförderung des Landes ist sehr stark auf mittelständische Betriebe ausgerichtet, damit diese neue Ideen zu marktfähigen Produkten entwickeln können.

Dieser kurze Überblick über die Handlungsfelder der Wirtschaftspolitik in Mecklenburg-Vorpommern verdeutlicht, wie stark die einzelnen Bereiche mit-

216

Vgl. der Wirtschaftsminister des Landes Mecklenburg-Vorpommern: "Tourismuskonzeption Mecklenburg-Vorpommern", Schwerin, 1993, S. 23/24.

217

Vgl. Landesregierung Mecklenburg-Vorpommern: "Konzeption zur Entwicklung ländlicher Raume in Mecklenburg-Vorpommern", Schwerin, 1992, S. 26.

Vgl. Landesregierung Mecklenburg-Vorpommern: "Konzeption zur Entwicklung lăndlicher Răume in Mecklenburg-Vorpommern", a.a.O., S. 11.

Unveröffentlichtes Grundsatzpapier der Technologiepolitik des Wirtschaftsministers des Landes Mecklenburg-Vorpommern aus dem September 1994. 
einander verbunden sind und sich gegenseitig ergänzen. Die Auswahl spiegelt die Struktur bzw. die Strukturprobleme des Landes wider und zeigt zugleich wirtschaftspolitische Schwerpunkte der Landesregierung auf. Die nun folgenden Abschnitte werden die im Rahmen dieser Handlungsfelder eingesetzten Mittel und Instrumente darstellen und analysieren.

\subsubsection{Wirtschaftsförderung des Landes}

Das Land Mecklenburg-Vorpommern hat insbesondere seit 1993 eigene Maßnahmen und Programme initiiert, die den oben beschriebenen vier Schwerpunkten zuzuordnen sind. Die Aktivitäten des Landes reichen von der "Beratung zum Anfassen" durch Senior-Experten über Bürgschaften und die Förderung der betrieblichen und außerbetrieblichen Ausbildung bis hin zum Landesinvestitionsprogramm zur Förderung von Unternehmen, die nicht die Kriterien der GA erfüllen.

Zunächst gebe ich einen Überblick über die Mittel, die für die unterschiedlichen Förderschwerpunkte zur Verfügung standen, anschließend werde ich die Programme des Landes darstellen und die wichtigsten näher analysieren.

\subsubsection{Mittel der Landesförderung}

Das Land Mecklenburg-Vorpommern versucht, mit seinen Landesförderprogrammen neben der GA eigene Schwerpunkte zu setzen sowie auf akute Probleme der Wirtschaft des Landes einzugehen. Diese Zielsetzung zeigt sich sehr deutlich in den Haushaltsansätzen für die landeseigene Wirtschaftsförderung (Tabelle 16). Die Tabelle stellt die Haushaltsansätze der für die Wirtschaftsförderung maßgeblichen Maßnahmengruppen der jeweiligen Jahre sowie deren Anteil an den Gesamtausgaben für Landesprogramme dar. Die Summe der veranschlagten Mittel stagniert zunächst auf niedrigem Niveau und steigt von 1993 bis 1995 auf 200 Mill. DM an. Der Rückgang der Mittel 1996 um 14\% verdeutlicht die angespannte Haushaltslage der öffentlichen Hand im allgemeinen und der neuen Länder im besonderen. Die Förderung des Landes konzentriert sich auf drei Schwerpunkte. Die berufliche Bildung in der Wirtschaft, die Förderung von Mittelstand, Handwerk, Handel und Dienstleistungen (im folgenden Mittelstandsförderung genannt) sowie die Förderung von Forschung, Entwicklung und Innovation.

Die Förderung der beruflichen Bildung hat zunächst einen hohen Stellenwert, stagniert dann jedoch 1992 und 1993 auf niedrigem Niveau. Im Jahr 1994 werden die Mittel im Vergleich zum Vorjahr mehr als verdoppelt und im Jahr 1995 mehr als verdreifacht. In diesen Zahlen spiegelt sich die Entwicklung auf dem Lehrstellenmarkt wider und sie zeigen sehr deutlich, wie flexibel die Landespolitik auf derartige Problemlagen reagieren kann. 
Tabelle 16

\begin{tabular}{|c|c|c|c|c|c|c|c|}
\hline \multicolumn{8}{|c|}{$\begin{array}{l}\text { Landesprogramme in Mecklenburg-Vorpommern } \\
\text { (Angaben in } 1000 \mathrm{DM} \text { ) }\end{array}$} \\
\hline & & 1991 & 1992 & 1993 & 1994 & 1995 & 1996 \\
\hline 1. & $\begin{array}{l}\text { Berufliche Bildung in } \\
\text { der Wirtschaft }\end{array}$ & $\begin{array}{r}23.200 \\
30 \%\end{array}$ & $\begin{array}{r}11.059 \\
15 \%\end{array}$ & $\begin{array}{r}11.755 \\
9 \%\end{array}$ & $\begin{array}{r}25.778 \\
18 \%\end{array}$ & $\begin{array}{r}82.697 \\
41 \%\end{array}$ & $\begin{array}{r}72.728 \\
42 \%\end{array}$ \\
\hline 2. & $\begin{array}{l}\text { Förderung des Frem- } \\
\text { denverkehrs }\end{array}$ & $\begin{array}{r}20.000 \\
26 \%\end{array}$ & $\begin{array}{r}17.700 \\
23 \%\end{array}$ & $\begin{array}{r}18.035 \\
13 \%\end{array}$ & $\begin{array}{r}8.709 \\
6 \% \\
\end{array}$ & $\begin{array}{r}8.104 \\
4 \% \\
\end{array}$ & $\begin{array}{r}6.610 \\
4 \% \\
\end{array}$ \\
\hline 3. & $\begin{array}{l}\text { Förderung von Mit- } \\
\text { telstand, Handel, } \\
\text { Handwerk und } \\
\text { Dienstleistungen }\end{array}$ & $\begin{array}{r}13.950 \\
18 \%\end{array}$ & $\begin{array}{r}11.600 \\
15 \%\end{array}$ & $\begin{array}{r}64.000 \\
48 \%\end{array}$ & $\begin{array}{r}69.250 \\
48 \%\end{array}$ & $\begin{array}{r}64.126 \\
32 \%\end{array}$ & $\begin{array}{r}49.348 \\
29 \%\end{array}$ \\
\hline 4. & $\begin{array}{l}\text { Werbemaßnahmen der } \\
\text { gewerblichen Wirt- } \\
\text { schaft }\end{array}$ & \begin{tabular}{r|r}
1.188 \\
$2 \%$
\end{tabular} & $\begin{array}{r}6.860 \\
9 \%\end{array}$ & $\begin{array}{r}11.169 \\
8 \%\end{array}$ & $\begin{array}{r}11.398 \\
8 \%\end{array}$ & $\begin{array}{r}4.553 \\
2 \%\end{array}$ & $\begin{array}{r}4.553 \\
3 \%\end{array}$ \\
\hline 5. & $\begin{array}{l}\text { Förderung von For- } \\
\text { schung, Entwicklung } \\
\text { und Innovation }\end{array}$ & $\begin{array}{r}9.000 \\
12 \%\end{array}$ & $\begin{array}{r}18.400 \\
24 \%\end{array}$ & $\begin{array}{r}18.340 \\
14 \%\end{array}$ & $\begin{array}{r}19.411 \\
13 \%\end{array}$ & $\begin{array}{r}30.180 \\
15 \%\end{array}$ & $\begin{array}{r}29.410 \\
17 \%\end{array}$ \\
\hline 6. & $\begin{array}{l}\text { Kooperation zwischen } \\
\text { Wissenschaft und } \\
\text { Wirtschaft }\end{array}$ & \begin{tabular}{r|r}
0 \\
$0 \%$
\end{tabular} & $\begin{array}{r}0 \\
0 \%\end{array}$ & $\begin{array}{r}0 \\
0 \%\end{array}$ & $\begin{array}{r}0 \\
0 \%\end{array}$ & $\begin{array}{r}0 \\
0 \%\end{array}$ & $\begin{array}{r}1.000 \\
1 \%\end{array}$ \\
\hline 7. & $\begin{array}{l}\text { Förderung von Betrie- } \\
\text { ben im Bereich mo- } \\
\text { dernerTechnologien }\end{array}$ & $\begin{array}{r}10.150 \\
13 \%\end{array}$ & $\begin{array}{r}10.013 \\
13 \%\end{array}$ & $\begin{array}{r}10.475 \\
8 \%\end{array}$ & $\begin{array}{r}10.575 \\
7 \%\end{array}$ & $\begin{array}{r}11.170 \\
6 \%\end{array}$ & $\begin{array}{r}9.350 \\
5 \%\end{array}$ \\
\hline & $\begin{array}{l}\text { Summe Landespro- } \\
\text { gramme }\end{array}$ & 77.488 & 75.632 & 133.774 & 145.120 & 200.830 & 172.998 \\
\hline
\end{tabular}

Um die drohende Lehrstellenknappheit zu bekämpfen, stellte das Land 199552 Mill. DM für Zuschüsse an private Unternehmen für die Schaffung von Ausbildungsplätzen in den Haushalt ein. Im Jahr 1996 wurden für diesen Zweck 44 Mill. DM vorgesehen. Die Dringlichkeit dieses Problems wird auch an dem Anteil von über $40 \%$ deutlich, den die Mittel für die berufliche Bildung 1995 und 1996 im Rahmen der Landesprogramme ausmachen.

Die Mittelstandsförderung zeigt eine wesentlich stetigere Entwicklung. In den Jahren 1991 und 1992 ist sie auf niedrigem Niveau annähernd konstant, steigt von 1993 bis 1994 im Zuge der allgemeinen Ausweitung der Landesprogramme auf über 69 Mill. DM an und fällt von 1995 bis 1996 auf unter 50 Mill. DM ab. Dieser Teil der Landesförderung dominiert mit $48 \%$ der Mittel in den Jahren 
1993 und 1994. Im Zuge der Mittelreduzierung und der Verschiebung von Schwerpunkten hin zur beruflichen Bildung und zur Förderung von Innovationen sank der Anteil der Mittelstandsförderung bis 1996 auf 29\% der Mittel.

Die Bedeutung der Förderung des Fremdenverkehrs verringert sich von 1991 bis 1996 sowohl absolut als auch relativ. Die verfügbaren Mittel gehen von 20 Mill. auf ca. 7 Mill. DM zurück, der Anteil an der Landesförderung von 26\% auf 4\%. Die Förderung des Fremdenverkehrs hat für Mecklenburg-Vorpommern eine herausragende Bedeutung, da der Tourismus insbesondere für die strukturschwachen Gebiete mittelfristig ein bedeutender Wirtschaftsfaktor sein kann und muß. Aufgrund der veränderten Förderbedingungen der GA, sind jedoch sehr viele Projekte in diesem Rahmen förderfähig, so daß das Land hier keine eigenen Schwerpunkte mehr setzen muß.

Der dritte Schwerpunkt, die Förderung von Forschung, Entwicklung und Innovation hat seinen Anteil in der Tendenz - abgesehen vom Jahr 1992 - von $12 \%$ auf $17 \%$ erhöht. Die Mittelausstattung ist relativ konstant. In den Jahren 1992-94 stehen ca. 19 Mill. DM zur Verfügung, 1995 und 1996 ca. 30 Mill. DM. Für die Förderung von Betrieben im Bereich moderner Technologien steht zwar über den gesamten Zeitraum ein relativ konstanter Betrag von ca. 10 Mill. DM zur Verfügung, jedoch nimmt die relative Bedeutung dieser Maßnahmengruppe von $13 \%$ der Mittel auf 5\% ab. Faßt man die beiden Maßnahmengruppen zusammen, so ist eine bewußte Innovations- bzw. Technologieorientierung zu erkennen. Die Förderung von Forschung, Entwicklung und Innovation zuzüglich der Förderung von Betrieben im Bereich moderner Technologien erreicht in allen Jahren einen Anteil von mindestens 20\%.

Die Zahlen verdeutlichen sehr gut die oben bereits angedeutete Ausrichtung der Landesförderung. Zum einen reagiert das Land auf akute wirtschaftspolitische Problemlagen, wie die Förderung der beruflichen Bildung deutlich zeigt. Zum anderen setzt es bewußt neben der GA eigene Schwerpunkte. Dies macht die nach wie vor bedeutsame Mittelstandsförderung sowie die Reduzierung der Tourismusförderung deutlich.

\subsubsection{Die Instrumente der Landesförderung}

Das Land Mecklenburg-Vorpommern bedient sich vielfältiger Instrumente zur Umsetzung seiner Wirtschaftspolitik. Die verschiedenen Programme und Maßnahmen, die 1994 zum Einsatz kamen, sind in Abbildung 5 dargestellt. Im Rahmen der Mittelstandsförderung werden im wesentlichen Unternehmen berücksichtigt, die nicht aus der GA gefördert werden können. Dies gilt für das Landesinvestitonsprogramm Teil I und II sowie für das Konsolidierungs- und das Investitionssicherungsprogramm. 
Abbildung 5

\begin{tabular}{|c|l|}
\hline \multicolumn{2}{|c|}{ Landeseigene Programme der Wirtschaftsförderung } \\
in Mecklenburg-Vorpommern 1994
\end{tabular}


Neben diesen die GA erweiternden Programmen setzt das Land eigene Schwerpunkte. Der Abbau von Managementdefiziten steht im Mittelpunkt der Beratung zum Anfassen. Im Rahmen dieses Programmes werden vornehmlich mittelständische Unternehmen durch Senior-Experten beraten. Die Imageförderung für einheimische Produkte setzt bei den vielfach beklagten Problemen ostdeutscher Anbieter an, mit ihren nicht selten qualitativ gleichwertigen Produkten auf dem westdeutschen Markt Fuß zu fassen. Die Programme der Fremdenverkehrsförderung ergänzen teilweise die GA (Förderung von Investitionsvorhaben und Infrastruktureinrichtungen). Der Schwerpunkt der Programme liegt allerdings eindeutig auf Bereichen, die von der GA nicht abgedeckt werden. Hierbei handelt es sich um die Verbesserung des Ausbildungsstandes der Mitarbeiter, die Entwicklung von Konzeptionen und die Werbung für das Urlaubsland MecklenburgVorpommern. Die Förderung der Berufsausbildung ist eine der originären Aufgaben des Landes. Ziel der Programme ist es, den Bedarf an betrieblichen und überbetrieblichen Ausbildungsplätzen in Mecklenburg-Vorpommern zu decken. Im Bereich der Technologie- und Innovationsförderung setzt das Land bewußt eigene Akzente. Im Zentrum der Förderung steht das Bestreben, mittelständischen Unternehmen in Mecklenburg-Vorpommern bei der Entwicklung neuer Technologien und der Einführung neuer, marktfähiger Produkte zu helfen.

Zusätzlich zu diesen vielfältigen Förderprogrammen existieren noch weitere Instrumente des Landes. Neben dem Anker Projekt, mit dem strukturbestimmende Treuhandbetriebe unterstützt werden, sind dies u.a. die Mittelständische Beteiligungsgesellschaft Mecklenburg-Vorpommern mbH sowie die Gesellschaft für Wirtschaftsförderung Mecklenburg-Vorpommern mbH. Das Land MecklenburgVorpommern bedient sich sehr unterschiedlicher Instrumente der Wirtschaftsförderung.

Nur ein Teil dieser Instrumente lehnt sich an die bestehenden Maßnahmen der GA an und deckt Förderlücken, die aus Sicht des Landes bestehen, ab. Der andere Teil der Maßnahmen setzt landesspezifische Schwerpunkte der Förderung und greift Anpassungsprobleme der Wirtschaft auf, die der Bund in seinen Programmen nur sehr eingeschränkt berücksichtigt.

\section{A. Das Landesinvestitionsprogramm (LIP)}

Das LIP ist ein landeseigenes Programm zur Ergänzung der GA. Es werden kleine und mittlere Unternehmen (KMU) gefördert, "... die wegen des fehlenden überregionalen Absatzes von der Förderung nach der Gemeinschaftsaufgabe 'Verbesserung der regionalen Wirtschaftsstruktur' ausgenommen sind". ${ }^{220}$ Ziel der Förderung ist die Schaffung von Dauerarbeitsplätzen und bzw. oder Ausbildungsplätzen.

Jahreswirtschaftsbericht Mecklenburg-Vorpommern 1994, S. 79. 
Gefördert werden nicht nur Unternehmen des produzierenden Gewerbes, sondern auch Unternehmen aus den Bereichen Handel, Handwerk und Dienstleistungen sowie die freien Berufe. Die Förderkonditionen gleichen denen der GA. Es wird ein Investitionszuschuß von $20 \%$ für die Errichtung, $15 \%$ für die Erweiterung und $12 \%$ für die Umstellung bzw. grundlegende Rationalisierung einer Betriebsstätte gewährt. Für das Programm, das 1993 erstmals aufgelegt wurde, waren im Haushalt 1993 zunächst 12,5 Mill. DM vorgesehen, die dann jedoch auf 64,5 Mill. DM aufgestockt wurden. Für das Jahr 1995 standen erneut 64,5 Mill. DM zur Verfügung. Für das LIP gibt es weder regionale noch sektorale Förderschwerpunkte.

Mit dem Förderkonzept des LIP geht das Land Mecklenburg-Vorpommern über den Ansatz der GA hinaus. Die Orientierung an der Exportbasistheorie und dem Konzept der Wachstumspole wird fallengelassen, und die Förderung wird bewußt auf die Betriebe ausgeweitet, die nicht zu den Basisaktivitäten gehören. Damit folgt das Land der Kritik an der Regionalförderung der GA. In den neuen Ländern und insbesondere in Mecklenburg-Vorpommern fehlen mittelständische Strukturen im Handwerk, im produzierenden Gewerbe sowie bei den freien Berufen. Diese mittelständischen Strukturen können neue Arbeitsplätze schaffen und Ausgangspunkt für überregional bzw. international tätige Unternehmen sein. Ferner ist für Industriebetriebe ein gut entwickeltes Handwerk ein nicht unbedeutender Standortfaktor. ${ }^{221}$

Es besteht eine wechselseitige Abhängigkeit zwischen mittelständischen Strukturen und der Ansiedlung von Industrie. Die ausschließliche Orientierung der GA an Basisaktivitäten muß daher aus Sicht des Landes um eine gezielte Förderung von mittelständischen Strukturen ergänzt werden. Dies ist für Mecklenburg-Vorpommern um so bedeutsamer, als die industrielle Basis bereits vor 1989 nicht sehr ausgeprägt war, und im Zuge des Anpassungsprozesses viele Industriearbeitsplätze verlorengegangen sind. Die Ansiedlung neuer Industriebetriebe ist kurz und mittelfristig trotz der hohen Förderung nicht in dem notwendigen Umfang möglich. Mittelständischen Strukturen kommt daher bei der Bereitstellung von Arbeits- und Ausbildungsplätzen eine wichtige Bedeutung zu. Dies unterstreicht auch die Entwicklung der Beschäftigten im Handwerk. Von 1989 bis 1993 nahm die Zahl der Beschäftigten von 25.704 auf $96.612 \mathrm{zu} .^{222}$

Vgl. hierzu trend GmbH: Zusammenfassung der Studie "Zum Stand und zur Entwicklung kleiner und mittelstăndischer Unternehmen des Handwerks", Studie im Auftrag der Handwerkskammer Schwerin, 1994, S. 10.

Wirtschaftsminister des Landes Mecklenburg-Vorpommern: "Der Mittelstand in Mecklenburg-Vorpommern", a.a.O., S. 46. 


\section{B Das Konsolidierungs und Investitionssicherungsprogramm}

Mit diesen beiden Programmen reagiert das Land auf Finanzierungsprobleme von Klein- und mittelständischen Unternehmen (KMU). In der Gründungsphase war die Eigenkapitalausstattung dieser Unternehmen nach Untersuchungen des Ifo Instituts und der Kreditanstalt für Wiederaufbau als gut zu bezeichnen. ${ }^{223} \mathrm{Im}$ Zuge der weiteren Unternehmensentwicklung nahm die Bedeutung von Finanzierungsproblemen für KMU jedoch zu. Ein Vergleich von KMU und Großunternehmen im Rahmen einer Umfrage des Instituts für Wirtschaftsforschung Halle macht diese Entwicklung sehr deutlich. Im April 1993 sahen 28\% der Großunternehmen, jedoch nur 16\% der KMU geringes Eigenkapital als ein Investitionshemmnis an. Im Mai 1994 hatte sich die Situation umgekehrt. Lediglich $18 \%$ der Großunternehmen, jedoch $36 \%$ der KMU klagten über zu geringes Eigenkapital. Auch bezüglich der Liquidität hat sich die Situation der KMU verschlechtert. Im Mai 1994 sahen 28\% der KMU mangelnde Liquidität als Investitionshemmnis an, bei Großunternehmen waren dies lediglich $14 \% .{ }^{224}$ Zahlen des Ifo Instituts aus dem verarbeitenden Gewerbe bestätigen diese Aussagen. Über Finanzierungsschwierigkeiten klagten 1991 nur $11 \%$ der Betriebe mit weniger als 50 Mitarbeitern, bei Betrieben mit mehr als 500 Mitarbeitern waren es $41 \%$. Auch hier kehrt sich die Situation um. Im Jahr 1994 haben 26\% der Betriebe unter 50 Mitarbeitern, jedoch nur 16\% der Betriebe mit mehr als 500 Mitarbeitern Finanzierungsschwierigkeiten. ${ }^{225}$

Aus Sicht des Landes sind diese Probleme "... in der mangelnden Kapitaldecke der Unternehmen, unzureichendem Know-how in der Führung des Unternehmens, in der Fehleinschätzung der Marktentwicklung sowie in der Fälligkeit ..."226 von zunächst tilgungsfreien Existenzgründerdarlehen begründet. Ein Teil der Probleme werde jedoch auch durch die restriktive Haltung der Banken verursacht. Diese würden eine Ausweitung von Kreditlinien verweigern und die Liquiditätsprobleme der Unternehmen durch teure Zwischenfinanzierungen tendenziell vergrößern. ${ }^{227}$

Ziel der Programme ist, Liquiditätsprobleme zu lösen und Investitionen strukturbestimmender Betriebe zu sichern. Im Rahmen des Konsolidierungsprogramms werden bedeutsame KMU der gewerblichen Wirtschaft sowie Angehö-

Vgl. hierzu Ifo Institut/IWH: "Finanzierungsprobleme kleiner und mittlerer Unternehmen der gewerblichen Wirtschaft in den neuen Bundesländern", München, Halle, 1994, S. 38.

Vgl. hierzu Ifo Institut/IWH: "Finanzienungsprobleme kleiner und mittlerer Unternehmen der gewerblichen Wirtschaft in den neuen Bundesländern", a.a.O., S. 30.

Vgl. hierzu Ifo Institut/IWH: "Finanzierungsprobleme kleiner und mittlerer Unternehmen der gewerblichen Wirtschaft in den neuen Bundesländern", a.a.O., S. 26.

Jahreswirtschaftsbericht Mecklenburg-Vorpommern 1994, S. 80.

Vgl. Jahreswirtschaftsbericht Mecklenburg-Vorpommern 1994, S. 80. 
rige der wirtschaftsnahen freien Berufe gefördert. Die Förderung soll in Regionen konzentriert werden, die durch schwerwiegende arbeitsmarktpolitische Probleme gekennzeichnet sind. Den Unternehmen wird durch eine Umfinanzierung von kurzfristigen, den Bestand gefährdenden Verbindlichkeiten in langfristige Verbindlichkeiten geholfen.

Mit dem Investitionssicherungsprogramm sollen regional bedeutsame KMU gefördert werden. Die Unternehmen müssen eine strukturelle Bedeutung für die Region haben und ihr Erhalt muß im gesamtwirtschaftlichen Interesse des Landes liegen. Förderfähig sind sowohl Investitionen, die bereits durch die GA oder das LIP gefördert wurden als auch neue Investitionen. Die Förderung erfolgt durch einen Investitionszuschuß.

Das Land versucht, mit diesem Programm Probleme des Transformationsprozesses abzufedern, die weder im Rahmen der GA noch des EFRE Berücksichtigung finden. Es möchte ein Wegbrechen von gerade gegründeten bzw. privatisierten Unternehmen verhindern, da dadurch der Anpassungsprozeß gestört, und der Bestand von Arbeitsplätzen gefährdet wird.

Die Zahlen über die Finanzierungsprobleme spiegeln zunächst einen Ausleseprozeß unter den neugegründeten bzw. übernommenen oder privatisierten Unternehmen wider, der in dieser Form durchaus hätte erwartet werden können. Insbesondere neue Unternehmen brauchen in der Gründungsphase eine höhere Eigenkapitalausstattung, um Anlaufverluste und Folgeinvestitionen tätigen zu können. Bleibt der Erfolg am Markt aus bzw. stellt sich dieser mit einer unerwartet langen Verzögerung ein, kommt es zu den oben beschriebenen Finanzierungsproblemen. Die Kreditinstitute haben bei der Allokation von Kapital eine wichtige Funktion. Es liegt nicht nur im Interesse der Anleger, sondern auch im Interesse der Volkswirtschaft, wenn die Allokation des Kapitals gemäß seiner Grenzleistungsfähigkeit erfolgt. Nur so ist sichergestellt, daß für aussichtsreiche und rentierliche Investitionen das nötige Kapital bereitgestellt werden kann. Der Zins, zu dem die Banken bereit sind, einem Unternehmen Geld zur Verfügung zu stellen, hängt von verschiedenen Faktoren ab. Er spiegelt unter anderem die Einschätzung über die Marktchancen eines Unternehmens und über die Vertrauenswürdigkeit eines Schuldners wieder. Die Banken werden ihrer gesamtwirtschaftlichen Allokationsfunktion somit durchaus gerecht, wenn sie von Unternehmen, die sich am Markt nicht durchsetzen können, höhere Zinsen verlangen oder diesen Unternehmen keine weiteren Kredite gewähren. Es ist fraglich, ob das Land im Zuge des Transformationsprozesses diesen Ausleseprozeß durch zusätzliche Liquiditätshilfen oder Investitionszuschüsse verhindern kann bzw. sollte. Dies ist insbesondere bei den Unternehmen fraglich, die sich trotz bereits erfolgter Förderung nicht durchsetzen können. Wie bei allen interventionistischen Förderprogrammen steht das Land bei dieser Programmkonzeption vor einem Informationsproblem, da es trotz gegenteiliger Marktsignale ein Unternehmen fördert und 
somit am Markt hält. Diese Entscheidung kann das Land eigentlich nur dann fällen, wenn es besser als der Markt in der Lage wäre, die Erfolgsaussichten eines Unternehmens zu beurteilen. Da die Förderentscheidung jedoch eine politische Entscheidung ist, die den Regeln der politischen Rationalität folgt, ist nicht anzunehmen, daß das Ergebnis der gemäß ökonomischer Rationalität gefällten Entscheidung des Marktes überlegen ist.

\section{Beratung zum Anfassen}

Ein wesentlicher Engpaßfaktor für Unternehmen in den neuen Ländern ist das nötige Management-Know-how zur Führung eines gewinnorientierten Betriebes in einer Marktwirtschaft. Der Bedarf an Information und Beratung war und ist nach wie vor qualitativ wie quantitativ so umfangreich, daß er nur eingeschränkt gedeckt werden kann. Die für die Beratung von Unternehmen wichtigen Steuerberater, Rechtsanwälte und Wirtschaftsprüfer waren in den neuen Ländern zunächst so gut wie gar nicht vorhanden.

Um den Prozeß des Wissenstransfers von West nach Ost zu beschleunigen und den Unternehmen günstige und professionelle Hilfe zu gewähren, fördert das Land den Einsatz von Senior-Experten zur Beratung von Unternehmen. ${ }^{228}$ Gefördert werden gewerbliche Unternehmen, Handwerksbetriebe, Betriebe des Großund Einzelhandels sowie die Freien Berufe durch einen Zuschuß von $80 \% \mathrm{zu}$ den in Rechnung gestellten Kosten.

Auch in Westdeutschland gab und gibt es öffentlich subventionierte Beratung. Deren volkswirtschaftliche Effizienz wird allerdings in Zweifel gezogen: "Die Kosten der Beratungsförderung dürften insgesamt den volkswirtschaftlichen Nutzen übersteigen. Öffentliche Beratungshilfen sind somit aus ökonomischer Sicht als ineffizient zu bewerten." ${ }^{229}$ Für dieses staatlich bezuschußte quasi ehrenamtliche Beratungsangebot gilt das Urteil von Ralf Nowak zur volkswirtschaftlichen Effizienz von subventionierter Beratung nicht. Zum einen arbeiten die Senioren-Experten nach den gleichen Prinzipien ohne staatliche Förderung seit einigen Jahren in Westdeutschland. Durch diese Vereine wird der umfangreiche Bestand an Wissen und Erfahrung, der bei ausgeschiedenen Managern vorhanden ist, jungen Existenzgründern und ratsuchenden Unternehmen zur Verfügung gestellt. Diese Art der Privatinitiative führt zu keinen Verzerrungen am Beratungsmarkt.

In den Senioren-Expertenorganisationen sind ehemalige Manager organisiert, die sich zum Ziel gesetzt haben, gegen eine Aufwandsentschädigung jungen Unternehmen ihr Know-how zur Verfugung zu stellen und ihnen mit Rat und Tat bei Problemen zu helfen.

Ralf Nowak: "Gesamtwirtschaftliche Aspekte von Existenzgrundungshilfen des Bundes und der Lănder", Köln, 1991, S.179. 
Im Zuge des Transformationsprozesses können die Senioren-Experten zu einem zügigen Know-how Transfer beitragen und somit die Transaktionskosten für ostdeutsche Unternehmen reduzieren. Je geringer die Kenntnisse über das neue Rechts- und Wirtschaftssystem sind, desto höher sind die Transaktionskosten für das einzelne Unternehmen. Aber auch gesamtwirtschaftlich sind diese Kenntnisse von Bedeutung, da nur so die transaktionskostensparende Wirkung von wirtschaftlichen Institutionen zum Tragen kommen kann.

\section{Das Anker-Projekt}

Mit dem Anker-Projekt reagiert die Landesregierung auf die Privatisierungspolitik der Treuhand Anstalt (THA). Um dieses Projekt richtig einordnen zu können, muß man zunächst kurz Ziele und Struktur der THA sowie ihre Politik betrachten.

Die Ziele der THA wurden im "Gesetz zur Privatisierung und Reorganisation des volkseigenen Vermögens" durch die Volkskammer der DDR am 17.06.1990 festgelegt. Interessant ist, daß die vorbereitende Arbeit für das Treuhandgesetz maßgeblich von der Regierung de Maiziéres bestimmt und gesteuert wurde. Als Diskussionsgrundlage für die Konzeption der THA diente nicht das Konzept aus dem Bundeskanzleramt und dem Wirtschaftsministerium ${ }^{230}$, sondern das sogenannte Bielefelder Konzept, daß von unabhängigen Persönlichkeiten erarbeitet wurde. ${ }^{231}$ Die Ziele der THA waren mehrdimensional und lassen sich wie folgt zusammenfassen:

a. Privatisierung und Verwertung des volkseigenen Vermögens nach den Grundsätzen der sozialen Marktwirtschaft

b. Förderung der Strukturanpassung an die Erfordernisse des Marktes (Entwicklung sanierungsfähiger Betriebe und wettbewerbsfähiger Unternehmen)

c. Herausbildung von marktfähigen Unternehmensstrukturen sowie einer effizienten Wirtschaftsstruktur durch Entflechtung

230 Dieses Konzept sah eine Aktiengesellschaft als Organisationsform der THA vor. Vgl. hierzu Wolfram Fischer, Harm Schröter: "Die Entstehung der Treuhandanstalt", in Wolfram Fischer/Herbert Hax/Hans Karl Schneider: "Treuhandanstalt. Das Unmögliche wagen", Berlin, 1993, S. 34.

231 Das Bielefelder Konzept wurde von den Professoren Hommelhoff und Krebs, dem Rechtsanwalt und Bankier Dr. Anneke und dem Bundesgeschäftsfuhrer des Wirtschaftsrates der CDU, Rudiger von Voss erarbeitet. Dieses Konzept sah einen offentlichrechtlichen Status der THA vor. Vgl. hierzu: Wolfram Fischer, Harm Schröter: "Die Entstehung der Treuhandanstalt", a.a.O., S. 34. 
Die THA hatte von Beginn an faktisch einen strukturpolitischen Auftrag, der weit über den reinen Privatisierungsauftrag hinausgingt. ${ }^{232}$ Als bundesunmittelbare Anstalt des öffentlichen Rechts hatte die THA weitgehende Entscheidungsund Handlungsbefugnisse. Sie war eng in das Geflecht der vorhandenen bzw. sich in den neuen Ländern entwickelnden politischen und wirtschaftlichen Institutionen eingebunden. Im Laufe ihrer Entwicklung wurden die kooperativen Verbindungen zu Ländern, Kommunen, Gewerkschaften sowie den Parlamenten immer komplexer. ${ }^{233}$ Die Einbindung der Länder wurde mit den "Grundsätzen zur Zusammenarbeit von Bund, neuen Ländern und Treuhandanstalt für den Aufschwung Ost" vom 15. März 1991 geregelt. Diese gaben ihnen über ihre Einflußmöglichkeit im Verwaltungsrat der THA hinaus erweiterte Informationsund Konsultationsrechte. ${ }^{234}$ Weitere Koordinierungsgremien waren die Treuhand-Wirtschaftskabinette, die Beiräte der Niederlassungen, die Montagsgespräche zwischen den Wirtschaftsministern der neuen Länder und Vertretern der THA sowie Branchen und Sanierungsgespräche.

Die strukturpolitische Bedeutung der THA läßt sich ferner an der Entwicklung ihrer Privatisierungspolitik ablesen. Es lassen sich drei Phasen unterscheiden: ${ }^{235}$

Phase 1: Privatisierung ist die beste Sanierung (bis Mitte 1991)

Phase 2: Ansanierung zur Privatisierungsvorbereitung (ab Mitte 1991)

Phase 3: Aktive Sanierung (1991/1992)

Im Rahmen der dritten Phase, im Zuge der Diskussion um den Erhalt von industriellen Kernen ${ }^{236}$, wurden zwischen einzelnen Ländern und der THA besondere

232 Vgl. hierzu auch Karl Lichtblau: "Privatisierungs- und Sanierungsarbeit der Treuhandanstalt", a.a.O., S. 31-36.

Diese Entwicklung wird sehr eindrucksvoll von Roland Czada analysiert: "Die Teuhandanstalt im politischen System der Bundesrepublik", in Aus Politik und Zeitgeschichte, B43-44/94, 28.10.1994, S. 31-42.

Vgl. Roland Czada: "Die Teuhandanstalt im politischen System der Bundesrepublik", a.a.O., S. 33 .

Vgl. hierzu Karl Lichtblau: "Privatisierungs- und Sanierungsarbeit der Treuhandanstalt", a.a.O., S. $36 \mathrm{ff}$. 
Koordinations- und Abstimmungsmechanismen vereinbart, um die Privatisierung bzw. Sanierung von strukturbestimmenden Treuhandbetrieben in enger Abstimmung vornehmen zu können.

Ziel des Anker-Projektes war, die "aktive und schnelle Sanierung der regional bedeutsamen Treuhandunternehmen...", um die "...Privatisierungschancen von sanierungsfähigen, aber seinerzeit nicht privatisierbaren Unternehmen zu erhöhen, indem ihnen Zeit und Mittel eingeräumt werden, zukunftsgerichtete Investitionen zu tätigen, marktfähige Produkte zu entwickeln oder neue Absatzgebiete $\mathrm{zu}$ erschließen." ${ }^{237}$ Hierzu wurde zwischen der Landesregierung und der THA eine Vereinbarung getroffen, die das Abstimmungsprozedere sowie die Beiträge der beiden Seiten bestimmt. Der Inhalt dieser schriftlich fixierten Vereinbarung sieht gemäß mir vorliegender Informationen aus der THA sowie dem Wirtschaftsministerium folgendes vor: Das Land benennt zunächst die strukturbestimmenden Betriebe, denen im Rahmen des Anker-Projektes geholfen werden soll. ${ }^{238}$ Die THA prüft, welche Betriebe aus ihrer Sicht sanierungsfähig sind und welche für die Liquidierung vorgeschlagen werden. Ist ein Betrieb nach Auffassung der THA sanierungsfähig, steht einer gemeinsamen Betreuung dieses Betriebes durch die THA und das Land nichts im Wege. Liegen allerdings unterschiedliche Auffassungen vor, so hat das Land die Möglichkeit, seine abweichende Position zu begründen. Die THA war grundsätzlich nicht bereit, die Mitfinanzierung von nach ihrer Ansicht nicht sanierungsfähigen Betrieben zu übernehmen. Das Land hatte jedoch die Möglichkeit, die Kosten einer Sanierung zu tragen, und somit eine Liquidierung abzuwenden. Die endgültige Entscheidung lag stets bei der THA.

Die wichtigste Funktion des Anker Projektes lag in der Betreuung der Betriebe. Dies bedeutete: $:^{239}$

Das Konzept der industriellen Kerne war nicht unumstritten, wurde jedoch sowohl von der THA, der Bundesregierung wie den Landesregierungen massiv unterstutzt. Von der Vielzahl der Beitrăge zu diesem Thema seien hier nur wenige exemplarisch angefuhrt: Hartmut Fest: "Vom Knacken industrieller Kerne", WD, 1993/IX, S. 469ff / Zeitgespräch im Wirtschaftsdienst zum Thema: "Erhaltung industrieller Kerne in Ostdeutschland?", mit Beitrăgen von Birgit Breul, Tyll Necker, Lothar Späth, Juergen Donges, WD 1993/II, S. 59ff / BMWi Außenstelle Berlin: "Konzept der Bundesregierung zur Sicherung und Erneuerung industrieller Kerne durch die Treuhandanstalt in den neuen Bundesländern", Pressemitteilung, Berlin, 1993 / BMWi, Bundeswirtschaftsminister Jürgen W. Mollemann zum Thema "Aktive Regional- und Strukturpolitik in den neuen Bundesländern", Pressemitteilung, Bonn 14.12.1992 / Dirk Nolte, Hartmut Tofaute: "Kahlschlag verhindern industrielle Kerne erhalten", WSI Materialien Nr. 36, Düsseldorf 1993. Jahreswirtschaftsbericht Mecklenburg Vorpommern 1994, S. 74.

Die Liste dieser Unternehmen war leider nicht zugänglich, da das Wirtschaftsministerium eine Veröffentlichung ablehnte.

Vgl. hierzu Jahreswirtschaftsbericht Mecklenburg Vorpommern 1994, S. 76. 
a. Identifizierung von Problem- und Rücknahmefällen und Feststellung der Sanierungsfähigkeit

b. Analyse und Überarbeitung des Unternehmenskonzeptes

c. Überarbeitung des Finanzierungskonzeptes

d. Nachverhandlungen mit der THA bezüglich der Vertrags- und Zahlungskonditionen

e. Fördermittelberatung

f. Initiierung von Qualifizierungs- und Beschäftigungsmaßnahmen

Der Erfolg des Anker-Projektes läßt sich nur sehr schwer messen. Nach Aussage des Wirtschaftsministeriums konnten von den 31 betroffenen Betrieben 17 privatisiert oder reprivatisiert werden und drei in Management KG's übernommen werden. Ferner wurden umfangreiche Sanierungs- und Abrißmaßnahmen durchgeführt, die die Privatisierung erheblich erleichterten. Das Wirtschaftsministerium bewertet das Anker-Projekt zusammenfassend sehr positiv: "Mit dem Anker-Projekt konnten wichtige industrielle Standorte in MecklenburgVorpommern erhalten werden. Diese strukturbestimmenden Betriebe sind Kristallisationskerne für eine wirtschaftliche Entwicklung unseres Landes. Diese Privatisierung ist auch ein Ergebnis der erfolgreichen Zusammenarbeit mit den Unternehmensverbänden und den Gewerkschaften im Anker-Beirat." ${ }^{240}$

Wie oben gezeigt, war die THA eine mit umfassenden Kompetenzen ausgestattete Einrichtung des Bundes. Sie übernahm die bestehenden Strukturen der DDR Wirtschaftsverwaltung und war zumindest in der Anfangsphase nur sehr eingeschränkt durch den Bundestag bzw. die Bundesregierung oder andere demokratisch legitimierte Gremien kontrollierbar. Die Länder erlangten ab 1991 zunehmend Einfluß auf die THA, der ihnen die Einbringung bzw. Durchsetzung ihrer regionalen Interessen ermöglichte. $\mathrm{Ob}$ die verstärkte Berücksichtigung regionaler Präferenzen bei den Entscheidungen der THA allerdings zu einer höheren allokativen Effizienz führte, ist nicht eindeutig zu klären. Für diese Annahme könnte die Übernahme der Sanierungskosten durch die Länder bei negativem Votum der THA sprechen. Für eine abschließende Bewertung der Wirkungen der institutionellen Verflechtungsstrukturen der THA ist eine umfassende Analyse ihrer Tätigkeit erforderlich. Hierzu ist allerdings eine uneingeschränkte Akteneinsicht notwendig, die selbst für die das Land Mecklenburg-Vorpommern betreffenden Akten nicht zu bekommen ist.

Daher können auch die im Rahmen des Anker-Projektes durchgeführten Maßnahmen nur eingeschränkt bewertet werden. Die Bündelung und Koordinierung der regionalpolitischen und arbeitsmarktpolitischen Programme verhindert Zielkonflikte zwischen diesen ansonsten wenig koordinierten Politikbereichen. Die

Jahreswirtschaftsbericht Mecklenburg Vorpommern 1994, S. 76. 
Beratung von notleidenden Unternehmen ist ähnlich zu bewerten wie die "Beratung zum Anfassen". Aufgrund der geringen betriebs- und volkswirtschaftlichen Kenntnisse bewirkt die staatlich geförderte Beratung von Unternehmen keine Verzerrung des Wettbewerbs, sondern schafft vielmehr die Bedingung für einen freien Wettbewerb.

Problematisch ist hingegen die Auswahl der strukturbestimmenden Betriebe. Zum einen ist der Begriff nicht klar definiert, zum anderen sind die Kriterien zur Auswahl der Betriebe nicht bekannt. Die Werftindustrie in MecklenburgVorpommern könnte als strukturbestimmend angesehen werden. Diese Abgrenzung ist allerdings statisch, da sie vom status quo ausgeht und nicht die Frage der Zukunftsperspektive eines Unternehmens berücksichtigt. Die Auswahl von strukturbestimmenden Betrieben birgt immer die Gefahr der Strukturkonservierung und die Behinderung von Wettbewerbsprozessen.

Andererseits ist es fraglich, ob der Wegfall vorhandener Industriestrukturen in einem industriell wenig entwickelten Land bei einem nur sehr eingeschränkten nationalen wie internationalen Ansiedlungspotential durch neue Industriestrukturen kompensiert werden kann. Die Erfahrung zeigt, daß Industrialisierungsprozesse nur über längere Zeiträume realisierbar sind. Das Land mußte daher eine politische Güterabwägung vornehmen zwischen dem Risiko, nicht wettbewerbsfähige Strukturen zu konservieren und dem Risiko des ersatzlosen Verlustes von Industrie. Unter den gegebenen Rahmenbedingungen ist das politische Risiko eines endgültigen Verlustes höher einzuschätzen als das Risiko der Strukturkonservierung. Dies liegt insbesondere an dem eingeschränkten Ansiedlungspotential, das u.a. durch die lohnpolitischen Weichenstellungen erheblich reduziert wurde. Für die wirtschaftspolitisch handelnden Landespolitiker war es daher aus ihrer Sicht rational, wenn sie sich für die Stützung industrieller Kerne einsetzten. Die Entwicklung dieser Kerne helegt jedoch, daß mit diesen Maßnahmen nur Zeit erkauft wurde, da die notwendigen Strukturanpassungen lediglich verschoben wurden. Dies wird ganz besonders im Werftenbereich deutlich.

\subsection{Zusammenfassung}

Voraussetzung für eine ordnungspolitische Regionalpolitik sind zentral gesetzte allgemeinverbindliche Regeln und Handlungsfreiräume der regionalen Einheiten. Die Analyse der institutionellen Strukturen zeigt, daß die Regeln der GA über die Grenzen des verflochtenen Systems hinaus nur eingeschränkt Bindungswirkung entfalten. Der Bund setzt keine allgemeinverbindlichen Regeln, da er keine Handhabe hat, die Bestimmungen der GA, die eigentlich auch Landesprogramme binden, durchzusetzen. Auch die Gemeinschaft nimmt die Funktion der Regelsetzung nicht wahr obwohl die Beihilfenkontrolle grundsätzlich diese Funktion übernehmen könnte. Beihilfen sind nach Ansicht der Kommission notwendig, um die Chancengleichheit zwischen regionalen Einheiten herzu- 
stellen. Die Kontrolle der Beihilfen basiert daher auf einem regionalpolitischen Konzept und dient schlußendlich nicht mehr der Durchsetzung allgemeinverbindlicher Regeln, sondern tendiert vielmehr zu Einzelfallentscheidungen, die sich an den regionalpolitischen Zielen und Leitbildern der Kommission orientieren. Sie basiert letztendlich auch auf einem konstruktivistischen Politikansatz und hat daher die gleichen Probleme wie die interventionistischen Programme der Gemeinschaft.

Auch die finanziellen Handlungsfreiräume des Landes MecklenburgVorpommern sind sehr eingeschränkt. Die Landesprogramme umfassen im Durchschnitt der Jahre 1990 bis 1996 12\% der gesamten Wirtschaftsförderung. Der Anteil der Zweckzuweisungen am Etat des Wirtschaftsministeriums beträgt im Durchschnitt $42 \%$ in der selben Periode. Die Dominanz der zentralen Handlungs- und Finanzierungskompetenz wird noch deutlicher, wenn man die Landesprogramme in Beziehung zu den vom Bund gesteuerten Förderprogrammen setzt. Alleine die Investitionszulage beläuft sich 1994 auf 4,82 Mrd. DM in den neuen Ländern. Der Netto-Investitionszuschuß der GA beläuft sich im selben Jahr lediglich auf 1,71 Mrd. DM. In Mecklenburg-Vorpommern entspricht die Höhe der landeseigenen Förderung im Durchschnitt 12\% der Förderung im Rahmen der GA. Im Lichte dieser Zahlen wird die geringe Dimension der landeseigenen Fördermaßnahmen und die Dominanz der zentral geplanten und gesteuerten Programme deutlich.

Das Land nutzt diese geringen Handlungsspielräume für eigene regional- und wirtschaftspolitische Schwerpunkte. Es konzentriert sich auf die Bereiche Mittelstandsförderung, Tourismusförderung, Technologiepolitik und Politik für den ländlichen Raum. Der Mittelansatz für diese Programme steigt von 77 Mill. DM 1991 auf 172 Mill. DM 1996.

Die Programme des Landes setzen eigene Akzente und lösen sich bewußt von dem theoretischen Fundament und den Vorgaben der GA. Einen Schwerpunkt der Landesförderung bildet z.B. die Unterstützung von Handwerk, freien Berufen und kleinen und mittelständischen Unternehmen. Diese fallen oftmals durch die Raster der GA, sind jedoch in einem industriearmen Land sehr bedeutsam für die Bereitstellung von Arbeits- und Ausbildungsplätzen. Ferner versucht das Land, den Zusammenbruch von jungen Unternehmen abzuwenden. Hierbei treten die typischen Probleme einer aktiven Industriepolitik auf, die versucht, die Ergebnisse des marktwirtschaftlichen Ausleseprozesses politisch zu korrigieren. In einem ähnlichem Dilemma befindet sich das Land gegenüber den Problembetrieben der THA. Das Land will industrielle Kerne erhalten, um damit das Wegbrechen von Industriearbeitsplätzen zu verhindern. Die handelnden Wirtschaftspolitiker stehen vor der Wahl, Industriearbeitsplätze möglicherweise endgültig zu vernichten oder durch Erhaltungssubventionen Strukturen zu konservieren. 
Die politische Rationalität spricht eindeutig für Erhaltungssubventionen. Die Empirie zeigt, daß damit allerdings nur Zeit erkauft wird, da die Strukturanpassungen unumgänglich sind. 
Wolfgang Grimme - 978-3-631-75004-9

Downloaded from PubFactory at 01/11/2019 09:23:21AM

via free access 


\section{Reform, Entwicklung und Reformfähigkeit der regionalpolitischen In- stitutionen}

Gegenstand der Analyse waren bisher die regionalpolitischen Institutionen und die in diesen Strukturen entwickelten und implementierten Programme in Mecklenburg-Vorpommern der Jahre 1990 bis 1994 . Wie im ersten Abschnitt herausgearbeitet, orientierte sich die Untersuchung an einem ordnungspolitischen Konzept der Regionalpolitik. Dies bedeutet, ausgehend von dem Hayekschen Wettbewerbskonzept, einen regelgebundenen Wettbewerb regionaler Einheiten um mobile Produktionsfaktoren. Für die Realisierung dieses Konzeptes ist eine bestimmte Kompetenzzuordnung in einem föderalen System eine notwendige Bedingung. Die Regelsetzung und Regeldurchsetzung muß auf zentraler Ebene angesiedelt werden, die konkreten Mittel-Zweck-Entscheidungen, also die Planung und Implementation von politischen Programmen, unterhalb dieser Ebene. Das Ergebnis der Analyse läßt sich wie folgt zusammenfassen:

a. Die praktizierten wirtschafts- und regionalpolitischen Konzepte und Programme lassen keine Orientierung an einer ordnungspolitischen Regionalpolitik erkennen. Die Regionalpolitik basiert auf konstruktivistischen Politikansätzen und versucht, regionale wirtschaftliche Entwicklungen mehr oder weniger zentral und leitbildorientiert zu steuern. Eine Regelsetzung wäre auf europäischer Ebene im Rahmen der Beihilfenkontrolle möglich, jedoch ist die Gemeinschaft selber regionalpolitischer Akteur, der eigene Ziele und $\mathrm{Ma}$ nnahmenprogramme verfolgt. Damit ist die Funktion der Setzung allgemeinverbindlicher Regeln nicht wahrnehmbar, da die Gemeinschaft dazu neigt, die Regeln einzelfallorientiert abzuändern.

b. Das Kriterium der fiskalischen Äquivalenz wird mehr oder weniger mißachtet. Das Land als Träger der Regionalpolitik ist im hohen Maße von zweckgebundenen Zuweisungen des Bundes und der EG bei der Finanzierung der regional- und strukturpolitischen Maßnahmen abhängig. Der Nutzen von geförderten Infrastrukturmaßnahmen und Investitionen der gewerblichen Wirtschaft fällt primär im Land bzw. in einzelnen Regionen an. Die Kosten werden jedoch im Rahmen der politischen Verflechtungsstrukturen auf alle Ebenen verteilt. Diese Situation bietet hohe Anreize für eine ineffiziente Allokation der Fördermittel und führt somit zu einer Verringerung der Effektivität.

c. Die Berücksichtigung regionaler Präferenzen ist in dem gegebenen institutionellen Rahmen nur sehr eingeschränkt möglich. Zielfindung, Programmentwicklung und -implementation erfolgen hauptsächlich von oben nach unten. Die Berücksichtigung regionaler Präferenzen ist eine Residualgröße, da sie nur insoweit zum Tragen kommt, wie die zentralen Instanzen Ent- 
scheidungsfreiräume eingeräumt haben. Auch gemessen an diesem Kriterium führen die institutionellen Rahmenbedingungen zu einer ineffizieten Allokation der Förderung und somit zu einer geringeren Effektivität.

Dieser ernüchternde Befund gibt Anlaß, die weitere Entwicklung nach $1994 \mathrm{zu}$ untersuchen und sich die Frage zu stellen, ob Reformen der regionalpolitischen Institutionen in Richtung einer ordnungspolitischen Regionalpolitik möglich sind. Da sowohl die GA, als auch der EFRE nach 1994, also außerhalb des Untersuchungszeitraumes, reformiert worden sind, sollen in diesem Abschnitt vier Fragen behandelt werden:

a. Wie müßte die europäische Regionalpolitik bzw. die GA verändert werden, damit eine ordnungspolitische Regionalpolitik möglich wird?

b. Wie sehen die tatsächlichen Veränderungen aus und wie sind sie zu bewerten?

c. Warum sind, was erwartbar ist, keine entscheidenden Schritte in Richtung einer ordnungspolitischen Regionalpolitik erfolgt?

d. Ist diese Reformblockade innerhalb der bestehenden politikökonomischen Strukturen aufhebbar oder müssen die politikökonomischen Rationalitäten verändert werden?

\section{Notwendige Reformen zur Einführung einer ordnungspolitischen Re- gionalpolitik}

Weder die Regionalpolitik der Gemeinschaft noch die GA orientieren sich an einem ordnungspolitischen Konzept der Regionalpolitik. Es gilt im folgenden herauszuarbeiten, welche Reformen notwendig wären, um eine ordnungspolitische Regionalpolitik zu ermöglichen. Zunächst stelle ich die allgemeinen Prinzipien einer solchen Reform dar, um dann im zweiten Schritt die notwendigen konkreten Veränderungen der europäischen Regionalpolitik und der GA darzustellen.

\subsection{Allgemeine Prinzipien einer ordnungspolitischen Regionalpolitik}

Erstes und wichtigstes Prinzip einer ordnungspolitischen Regionalpolitik ist der regelgebundene Wettbewerb. Dies erfordert die zentrale Setzung- und Durchsetzugn von „rules of just conduct“, also von allgemeinverbindlichen Regeln, die den Wettbewerb der Regionen binden. Diese Funktion kann in dem existierenden föderalen System Europa nur von der Gemeinschaft wahrgenommen werden. Zweites Prinzip ist die Regionalisierung der Entscheidungs- und Finanzierungskompetenzen, die sich an dem Prinzip der fiskalischen Äquivalenz und der Berücksichtigung regionaler Präferenzen orientieren sollte. 
Dieses Prinzip wird auch in der Literatur von verschiedenen Autoren vertreten. ${ }^{240}$ So fordert van Suntum eine Dezentralisierung des öffentlichen Sektors: "Im öffentlichen Sektor besteht das Grundproblem regionaler Effizienz darin, zum einen in allen Teilregionen eine möglichst gute Übereinstimmung zwischen den Präferenzen der dortigen Bevölkerung und dem regionalen öffentlichen Güterangebot zu gewährleisten, zum anderen dafür zu sorgen, daß die Wirtschaftssubjekte sich tendenziell dort ansiedeln, wo ihre Versorgung mit öffentlichen Gütern zu den langfristig geringsten Kosten erfolgen kann. Beide Anliegen sprechen für eine Dezentralisierung der fiskalischen Gewalt." ${ }^{141}$ Auch bei Konrad Lammers ${ }^{242}$ sowie Martin Junkernheinrich findet sich dieser Ansatz. Martin Junkernheinrich stellt fest: "Bei wettbewerbsorientierten Rahmenbedingungen vermag eine solche Regionalisierung der Regionalpolitik das Eigeninteresse der Teilräume und die gesamtwirtschaftliche Effizienz miteinander in Einklang zu bringen." ${ }^{1243} \mathrm{Ganz}$ deutlich arbeitet Herbert Giersch in seiner „Thünen Vorlesung“ die Bedeutung des Wettbewerbs heraus. In diesem Wettbewerb, so Giersch, werden "... die Attraktivität der bisherigen Ressourcenausstattung und die lokalen öffentlichen Güter, einschließlich der sozialen Atmosphäre mit ihren ethischen Normen und mit den Zwängen und Leistungen die von der öffentlichen Hand ausgehen..."244 eingesetzt. Der Wettbewerb der Standorte hatte für Europa seit Beginn der Neuzeit eine herausragende Bedeutung. Ohne diesen Wettbewerb "... hätte der alte Kontinent wahrscheinlich nicht soviel an Eigentumsrechten entstehen lassen, wie notwendig war, um seine Vielfalt in evolutorische Arbeitsteilung umzusetzen. Dies war, wie heute zunehmend erkannt wird, das „Wunder Europa." 245

Neben den hier zitierten Autoren wird dieser Ansatz u.a. auch noch von Paul Klemmer ("Regionalpolitik auf dem Prufstand", Köln 1986) sowie von Bernd Spiekermann ("Europäische Regionalpolitik", Koln 1988) vertreten.

Ulrich van Suntum: "Regionalpolitik in der Marktwirtschaft", a.a.O., S. 137.

Vgl. hierzu Konrad Lammers: "Wege der Wirtschaftsforderung für die neuen Bundesländer", Die Weltwirtschaft, 1990, Heft 2, S. 106ff.

Martin Junkernheinrich: "Răumliche Wachstumspolitik in den neuen Bundeslăndern Möglichkeiten einer konzeptionellen Ausgestaltung", in G. Braun, D. Gerlach: "Probleme der Regional- und Strukturpolitik in Mecklenburg-Vorpommern, Rostock, 1994.

Herbert Giersch: "Thunen-Vorlesung", Zeitschrift fur Wirtschafts- und Sozialwissenschaften, 110 (1990), S. 3.

Herbert Giersch: "Thunen Vorlesung", a.a.O., S. 3. Giersch bezieht sich hier ausdrücklich auf E.L. Jones, "The European Miracle", Cambridge, 1981. 
Damit die positiven Effekte einer optimalen Dezentralisierung regional- und strukturpolitischer Kompetenzen realisiert werden können, müssen allerdings noch andere institutionelle Strukturen verändert werden. Bedingung für eine optimale Dezentralisierung ist eine Reform der Finanzverfassung mit dem Ziel, den Ländern bzw. den Kommunen unabhängig von zentralstaatlichen Einflüssen Dispositionsfreiheit über die für ihre Aufgaben ausreichenden Einnahmen zu geben. Für die Ausgestaltung einer solchen Reform gibt es jedoch sehr unterschiedliche Ansätze. ${ }^{246}$ Damit der öffentliche Sektor bezüglich der räumlichen Faktorallokation neutral ist, fordert van Suntum, in Anlehnung an Buchanan die Orientierung am Prinzip der fiskalischen Gleichheit bzw. die Besteuerung nach dem Äquivalenzprinzip. ${ }^{247}$ Fiskalische Gleichheit ist erreicht, wenn "... the balance between the contributions made and the value of public services returned to the individual..."248 in allen Teilregionen für ein gegebenes Wirtschaftssubjekt gleich ist. Aus diesem Ansatz folgt, daß redistributive Steuerquellen nur dann bezüglich der räumlichen Allokation neutral sind, wenn sie "... den Teilregionen nach der Zahl der dort ansässigen Wirtschaftssubjekte zugeteilt werden." ${ }^{249}$ Sollten für die Länderebene eigene Finanzierungsquellen vorgesehen werden, so müßten sich diese am Äquivalenzprinzip orientieren und somit dürfte "... die Finanzierung über Gebühren und Beiträge statt durch Steuern in diesem Bereich vorzuziehen sein."250 Für die Kommunen lehnt van Suntum z.B. die Einführung eines eigenen Hebesatzrechtes auf die Einkommenssteuer ab. ${ }^{251}$ Er präferiert vielmehr die Verteilung des Steueraufkommens gemäß der Einwohnerzahl, da so eine den regionalpolitischen Erfordernissen genügende Lösung gefunden wäre. ${ }^{252}$

Zur Reform der Gemeindefinanzen seien hier exemplarisch aus der jüngsten Zeit ein paar Publikationen genannt, die die Diskussion widerspiegeln: Martin Kinkel: "Abschaffung der Gewerbekapitalsteuer und ihre Kompensation", WD 1995/V, S. 250ff / Martin Junkernheinrich: "Reform des Gemeindefinanzsystems - Zur Position des Sachverständigenrates zur Begutachtung der gesamtwirtschaftlichen Entwicklung", in Archiv fur Kommunalwissenschaften, II/1992, S. 220 ff. / P. Klemmer, M. Junkernheinrich: "Neuordnung des Gemeindefinanzsystems", Berlin 1994, Band 3 der Reihe "Forum Öffentliche Finanzen".

Vgl. hierzu Ulrich van Suntum: "Regionalpolitik in der Marktwirtschaft", a.a.O., S. 102 ff.

James M. Buchanan: "Federalism and Fiscal Equity", American Economic Review, Vol. 40, S. 588.

Ulrich van Suntum: "Regionalpolitik in der Marktwirtschaft", a.a.O., S. 110.

Ulrich van Suntum: "Regionalpolitik in der Marktwirtschaft", a.a.O., S. 110.

Ulrich van Suntum: "Regionalpolitik in der Marktwirtschaft", a.a.O., S. 190.

Ulrich van Suntum: "Regionalpolitik in der Marktwirtschaft", a.a.O., S. 191. 
Mit dieser Position befindet er sich im Widerspruch zu Alfred Boss und Konrad Lammers. Konrad Lammers fordert zur Stärkung der kommunalen Steuerhoheit die Einführung einer Wertschöpfungssteuer. ${ }^{253}$ Auch für die Länder empfiehlt Lammers eine eigene Steuerhoheit. Den Ländern könnte, so Lammers, die Möglichkeit eingeräumt werden, "... einen Zuschlag zur Einkommen- und Körperschaftssteuer zu erheben." 254 Alfred Boss plädiert hingegen dafür, den Kommunen ein Hebesatzrecht auf die Einkommen- bzw. Körperschaftssteuer einzuräumen. In dieser Lösung sieht er u.a. die Chance, daß sich die Besteuerung an den kommunalen Präferenzen orientieren kann und die Transparenz von ausgabenwirksamen kommunalen Maßnahmen deutlicher wird. ${ }^{255}$

Desweiteren müssen die Mischfinanzierungstatbestände abgebaut werden. ${ }^{256}$ Wie die Analyse gezeigt hat, führen Mischfinanzierungen zu Fehlallokationen, da die regionalen Präferenzen verwischt werden und das Prinzip der fiskalischen Äquivalenz in aller Regel verletzt wird. Theoretisch können Mischfinanzierungen zur Übereinstiummung von Kosten und Nutzen eines öffentlichen Gutes beitragen. So können die positiven externen Effekte eines auf kommunaler Ebene bereitgestellten öffentlichen Gutes durch Zuweisungen aus zentralen Haushalten ausgeglichen werden, so daß die Kommune nur den ihrem Nutzen entsprechenden Teil der Kosten trägt. Tatsächlich kommt es jedoch zu einer Ausdehnung von Mischfinanzierungstatbeständen über das ökonomisch effiziente Maß hinaus, da dies im Interesse der politischen Akteure liegt. Die dezentrale Einheit versucht, im Rahmen der Budgetmaximierung möglichst viele Zuweisungen von der zentralen Einheit zu erlangen. Dieses rent-seeking-Verhalten kommt dem Interesse der zentralen Einheit durchaus entgegen. Sie möchte nämlich über die Zuweisungen Einfluß auf die Entscheidungen der politischen Akteure in den dezentralen Einheiten gewinnen und somit ihre politische Legitimation sichern. Diese Interessenkonstellation führt dann zu einer de facto Zentralisierung der Zuständigkeiten.

253 Vgl. hierzu Konrad Lammers: "Wege der Wirtschaftsforderung für die neuen Bundesländer", a.a.O., S. 107/108. Eine ăhnliche Position vertritt auch der Sachverstăndigenrat in seinem Jahresgutachten 1986 sowie der wissenschaftliche Beirat beim Bundesfinanzministerium.

Konrad Lammers: "Wege der Wirtschaftsforderung für die neuen Bundesländer", a.a.O., S. 108.

Vgl. hierzu Alfred Boss: "Für eine kommunale Einkommensteuer", FAZ vom 16.12.1994.

Vgl. hierzu Konrad Lammers: "Wege der Wirtschaftsforderung für die neuen Bundesländer", a.a.O., S. 108. 
Ferner müssen die Geldleistungsgesetze überarbeitet werden. ${ }^{257}$ Es widerspricht dem Prinzip der fiskalischen Äquivalenz, wenn der Bund über den Umfang von Leistungen beschließt und die Zahlungsverpflichtung den Ländern bzw. den Kommunen aufbürdet. ${ }^{258}$

\subsubsection{Ordnungspolitische Regionalpolitik in der Gemeinschaft und in Deutschland}

Eine ordnungspolitische Regionalpolitik der Gemeinschaft müßte basierend auf der in Art 92 bis 94 EWGV verankerten Beihilfenkontrolle allgemeinverbindliche Regeln für einen Wettbewerb der regionalen Einheiten definieren. Damit diese Regeln die Entstehung einer Wettbewerbsordnung unterstützen können, müssen sie einen verläßlichen und zumindestens mittelfristig feststehenden Koordinierungsrahmen bieten. Die Regeln dürfen daher nicht Ergebnis einer fortlaufenden Interpretation der relevanten Artikel des EWG-Vertrages sein, da dies im Ergebnis zu einer Anpassung der Regeln an aktuelle Einzelfallentscheidungen verleitet. $\mathrm{Zu}$ welchen Ergebnissen ein derart offener Prozeß führt, hat die laufende Veränderung der Beihilfenkontrolle in den letzten Jahren gezeigt. Aber auch aus der Wissenschaft werden immer wieder neue Vorschläge zur Abgrenzung der Beihilfen beigetragen, unter anderem werden folgende Erweiterungen des Beihilfenbegriffs vorgeschlagen: ${ }^{259}$

- $\quad$ Erfassung von Beihilfen öffentlicher Unternehmen

- $\quad$ Einbeziehung von Bebauungsplanänderungen, durch die Unternehmen höhere Verkaufserlöse erzielen. Hierbei muß der Begünstigungszweck allerdings Ziel der Änderung sein

- Veränderungen nationaler Steuervergünstigungen. Wobei als Beihilfe die "... Abweichungen von der jeweiligen nationalen Steuernorm ..." gewertet wer-

Vgl. hierzu Konrad Lammers: "Wege der Wirtschaftsförderung für die neuen Bundesländer", a.a.O., S. 108.

Auch die im Rahmen des Schwangerenhilfegsetzes den Kommunen auferlegte Pflicht zur Schaffung von Kindergartenplätzen ist unter diesem Aspekt als sehr problematisch zu bewerten. Für den Bereich der Sozialhilfe gibt es allerdings auch Vorschlage, die die Entscheidung uber Umfang und Hơhe der Hilfe den Kommunen uberlassen wollen. Vgl. hierzu: Alfred Boss, Peter Trapp: "Sozialhilfe ist Sache der Gemeinden", FAZ vom 30.05.1995.

Vorschlăge zu einer Ausweitung des Beihilfenbegriffs finden sich bei Gisela Fărber: "Binnenmarktgerechte Subventionspolitik in der Europăischen Union", Campus Verlag, 1995, S. 420. 
den sollen, die "... die Unternehmen besser stellen als es der ökonomische Sachverhalt erfordern würde" 260

Der hier eingeschlagene Weg hat nur noch wenig mit generellen, allgemeinverbindlichen Regeln zu tun. Er folgt vielmehr dem bürokratischen Verlangen, eine möglichst umfassende Erfassung aller Beihilfen sicherzustellen. Von diesen hier vorgeschlagenen Erweiterungen ist es nicht mehr weit zu einer beihilferechtlichen Überprüfung von grundlegenden ordnungspolitischen Weichenstellungen, die im Wettbewerb der Systeme einen Vorteil darstellen. ${ }^{261}$ Allgemeinverbindliche Beihilferegeln, die die Entstehung einer Wettbewerbsordnung für den Wettbewerb der regionalen Einheiten unterstützen sollen, müssen vielmehr so formuliert sein, daß im Rahmen der Regeln unterschiedliche ordnungs- und wirtschaftspolitische Entscheidungen möglich sind.

Um die Funktion der Regelsetzung und Regeldurchsetzung übernehmen zu können, muß die Gemeinschaft auf alle konkreten Maßnahmenprogramme verzichten. Die Zuständigkeit für derartige Programme sollte bei den regionalen Einheiten oder den Mitgliedstaaten angesiedelt werden. Konkret bedeutet dies die Auflösung aller Strukturfonds sowie die des Kohäsionsfonds. Ausgleichspolitischer Handlungsspielraum blieb der Kommission bzw. dem europäischen Rat über die konkrete Ausgestaltung einer europäischen Finanzverfassung bzw. eines europäischen Finanzausgleichs, der über ungebundene Transfers eine Angleichung der finanziellen Leistungsfähigkeit in der EG herbeiführt. Eine europäische Finanzverfassung müßte so strukturiert sein, daß sie die ordnungspolitische Grundsatzentscheidung für einen Wettbewerb der Regionen unterstützt. Dies bedeutet zunächst, daß grundsätzlich die Handlungs- und Finanzierungskompetenz in einer Hand liegen muß. Die Ebene, die Programme beschließt, muß diese auch aus ihrem Budget finanzieren. Diese Regel reduziert die Anreize zu einem Förderwettbewerb, da jedes Förderprogramm zu 100\% aus dem eigenen Budget finanziert werden muß. Desweiteren kann eine europäische Finanzverfassung über bestimmte Budgetristriktionen die Entstehung einer Wettbewerbsordnung unterstützen. Dies könnten zum Beispiel die derzeit im Rahmen des Paktes für Stabilität und Wachstum vor-

Gisela Färber: "Binnenmarktgerechte Subventionspolitik in der Europäischen Union", a.a.O., S. 421 .

Auch Martin Junkernheinrich und V. Nienhaus kommen zu diesem Ergebnis. Junkernheinrich beschreibt die Konsequenz eines solchen Politikansatzes wie folgt: "The consequence would be no longer national institutions and measures competing among each other, but rather all kinds of political areas brought in harmony with each other. In the strictest sense of the idea, the EC even would have to disallow tax exemption, deregulation, debureaucratisation and other improvements in the regional economic condition." M. Junkernheinrich: "Europaenisation of Regional Policy? - Supranational impact on German Regional Policy", IWH Diskussionspapier Nr. 15, Mai 1994, S. 16. 
geschlagenen Verschuldungs- und Defizitbegrenzungen sein. Damit ist den Mitgliedsstaaten und Regionen die Möglichkeit genommen, über ein erhöhtes Haushaltsdefizit Beihilfen auszuweiten, ohne andere Politikbereiche einzuschränken bzw. zusätzliche Einnahmen durch höhere Steuern oder Abgaben zu erzielen. Somit sind die Kosten bestehender und jeder weiteren Beihilfe sowie deren Nutzen durch den jeweiligen Staat bzw. die jeweilige Region zu tragen. Die regionalen politischen Entscheidungsträger haben ein Interesse daran, ein ausgewogenes Budget vorzulegen, in dem die wichtigsten Politikfelder ausreichend berücksichtigt werden. Eine Ausweitung von Beihilfen zu Lasten anderer Bereiche könnte zu einem Verlust von Wählerstimmen führen. Dies führt dazu, daß der politische Wettbewerb um Wählerstimmen "... is thus in much greater agreement with overall economic efficiency than the present centralist structure would ever allow, where the exploitation of anonymous funds, i.e., the economically most inefficient behaiviour, is politically rewarded." ${ }^{262}$

Ein Ziel der deutschen Regionalpolitik im Rahmen der GA ist es, einen Förderwettbewerb der Länder zu verhindern. Im Rahmen des Binnenmarktes und einer ordnungspolitischen Regionalpolitik ist die Zuordnung dieser Funktion auf nationaler Ebene nicht adäquat. Diese Aufgabe obliegt, wie oben ausgeführt, der Gemeinschaft. Die GA der bisherigen Ausprägung kann eine ordnungspolitische Regionalpolitik nicht umsetzen, da der intenventionistische leitbildorientierte Politikansatz einem regelgebundenen Wettbewerb der regionalen Einheiten entgegensteht. Die GA muß daher abgeschafft und die Kompetenzen, die bisher im Rahmen dieser verflochtenen Struktur wahrgenommen wurden, müssen regionalisiert werden. Insbesondere für die Bundesrepublik Deutschland gilt, daß die ausgleichspolitische Zielsetzung des Grundgesetzes über eine entsprechende Gestaltung der Finanzverfassung realisiert werden kann. Um eine ordnungspolitische Regionalpolitik zu realisieren, bedarf es jedoch Reformen in der derzeit gültigen Zuordnung der Entscheidungs- und Finanzierungskompetenzen. Ziel einer solchen Reform muß es sein, die Finanzierungskompetenz dort anzusiedeln, wo die Handlungskomptenz liegt. Mischfinanzierungen und Zweckzuweisungen sollte es nur noch insoweit geben, wie sie mit der Internalisierung externer Effekte begründet werden können.

Unter der Bedingung, daß die Finanzverfassung entsprechend dieser Grundsätze reformiert wird, und die dezentralen Einheiten autonom über ihre Ausgaben und zumindestens teilweise über eigene Einnahmequellen verfügen, können die regionalpolitischen Kompetenzen effizienter und effektiver auf dezentraler Ebene wahrgenommen werden. Die Tatsache, daß eine Ausweitung von Förderprogrammen bzw. Fördergebieten nur zu Lasten anderer Ausgaben möglich ist, verman Regional Policy", a.a.O., S. 18. 
hindert die Einführung von Förderprogrammmen über das ökonomisch effiziente Maß hinaus. In dieser Situation hat die dezentrale Einheit Art und Umfang der Förderung alleine $\mathrm{zu}$ verantworten und trägt somit auch die Konsequenzen einer verfehlten Förderpolitik. Auch hier gilt, daß das Ziel der Stimmenmaximierung den ökonomischen Effizienzkriterien mehr zur Geltung verhilft als die bestehenden Verflechtungsstrukturen.

Gegen die Beibehaltung der derzeitigen regionalpolitischen Konzeption sprechen jedoch auch die regionaltheoretischen Defizite. Der quantitativ interventionistische Politikansatz der GA kann nur dann rational umgesetzt werden, wenn die verschiedenen regionalen Produktionsfunktionen bekannt sind. Nur dann läßt sich ein zieladäquater Einsatz der Mittel und Instrumente steuern. Diese Funktionen gibt es weder für die alten noch für die neuen Länder. Für die neuen Länder kommt erschwerend hinzu, daß der Transformationsprozeß und seine regionalpolitischen Auswirkungen theoretisch nur sehr wenig ausgeleuchtet sind. Ferner fehlt vielfach die notwendige Qualität und Quantität der Daten, um verläßliche Untersuchungen durchzuführen. Das ohnehin vorhandene Informationsproblem für eine zentrale Planungseinheit war und ist in dieser Situation erheblich verschärft. Daher spricht die besondere Situation in den neuen Ländern nicht für eine zentrale Steuerung und Kontrolle der Regionalpolitik, sondern für die bewußte Dezentralisierung der Zuständigkeiten und die Steuerung mit ordnungspolitischen Instrumenten. $\mathrm{Fa} 3 \mathrm{t}$ man die theoretischen Überlegungen zusammen, so wird deutlich, daß eine Dezentralisierung der regionalpolitischen Aufgaben und die Abschaffung der GA die Effizienz und Effektivität der Förderung erheblich erhöhen würde.

Welche Kompetenzen dann auf welcher Ebene des föderalen Systems anzusiedeln sind, bedarf eingehender Analysen. Die Zuordnung sollte sich an dem Kriterium der fiskalischen Äquivalenz orientieren, regionale Präferenzen berücksichtigen sowie Wettbewerbsprozesse ermöglichen. Im Ergebnis können bestimmte regionalpolitische Kompetenzen auch auf der kommunalen Ebene angesiedelt werden. Ein Großteil der Förderung der wirtschaftsnahen Infrastruktur kann z.B. effizienter durch die Kommunen bzw. durch regionalen Planungsverbände geplant und vergeben werden.

\subsection{Entwicklung der regionalpolitischen Institutionen}

Der folgende Abschnitt dient der Gegenüberstellung der oben theoretisch entwikkelten Reformvorschläge und der tatsächlichen Entwicklung der regionalpolitischen Institutionen. Hierbei kommt es mir nicht auf eine umfassende und detaillierte Analyse neuer Programme und Verordnungen an. Ich möchte vielmehr die Entwicklungstendenz aufzeigen, die sich aus den institutionellen Veränderungen ergibt. 


\subsubsection{Die Reform der EG Strukturfonds}

Die seit 1989 gültige Fondsverordnung sollte fünf Jahre nach ihrem Inkrafttreten überprüft werden ${ }^{263}$ Neben der Revisionsklausel in der Verordnung machten die Beschlüsse der Gipfeltreffen von Maastricht, Lissabon und Edinburgh eine Anpassung der Fondsverordnung erforderlich. Kern der Gipfelbeschlüsse war eine erhebliche Aufstockung der Mittel für die Strukturfonds sowie die Einrichtung des Kohäsionsfonds. Für die Jahre 1994 bis 1999 betragen die gesamten Mittel ca. 155 Mrd Ecu, ${ }^{264}$ für den Zeitraum 1989 bis 1993 standen lediglich 63 Mrd Ecu zur Verfügung. ${ }^{265}$ Die vier Grundpfeiler der Verordnung von 1989, die Orientierung an Zielen, der koordinierte Einsatz der unterschiedlichen Fonds, die Partnerschaft sowie die Programmfinanzierung bleiben erhalten und werden teilweise ausgebaut bzw. verändert.

Die Ziele der Strukturfonds werden tendenziell ausgedehnt. Neu aufgenommen werden u.a. Gebiete, die vom Strukturwandel im Fischereisektor negativ betroffen sind und städtische Regionen, die schwerwiegende Probleme mit Industriebrachen und Altlasten haben. ${ }^{266}$ Die Verknüpfung von regionalpolitischen Zielen mit sozial- und gesellschaftspolitischen Zielen wurde in der neuen Verordnung verstärkt. Insbesondere das Europäische Parlament stellt diese Entwicklung als besonders positiv heraus und fordert weitere Schritte in diese Richtung. Die Sozialpartnerschaft ${ }^{267}$ bei der Entwicklung und Durchführung der Programme soll gestärkt werden und die Chancengleichheit von Mann und Frau und umweltpolitische Ziele sollen berücksichtigt werden. ${ }^{268}$

Die Fördergebiete werden ausgeweitet. Neben den neuen Bundesländern werden weitere sechs Regionen in die Förderung gemäß Ziel 1 aufgenommen, "... von denen die meisten ihren Status rein politischen Erwägungen verdanken, da sie

Vgl. Verordnung (EWG) Nr. 2052/88 des Rates vom 24.06.1988, Art. 19 in ABl. Nr. L $185 / 9$ vom 15.07 .1988 .

Verordnung (EG) Nr. 2081/93, ABl. (EG) L 193 vom 31.07.1993, Anhang II.

EG-Kommission: "Leitfaden zur Reform der Strukturfonds in der Gemeinschaft", Luxemburg 1989, S. 18.

Vgl. Roland W. Waniek: "EG-Regionalpolitik fur die Jahre 1994 bis 1999", WD 1994/I, S. $44 / 45$.

Die Sozialpartnerschaft sieht die Beteiligung der Sozialpartner bei der Aufstellung und Durchfuhrung der Regionalentwicklungsprogramme vor.

Vgl. Hartmut Hausmann: "Vom Mauerblümchen zum wichtigsten Finanzinstrument" in: Das Parlament vom 30.07.93. Zitiert werden in diesem Artikel die Berichterstatter der groBen Fraktionen des Parlaments fur die Strukturfonds. 
oberhalb des Schwellenwertes von 75\% des EG-Durchschnitts beim BIP je Kopf liegen...". ${ }^{269}$ Der Anteil der Bevölkerung, der in Ziel-1-Regionen wohnt, steigt von $21,7 \%$ auf 26,6\%. Rechnet man noch den maximal erlaubten Bevölkerungsanteil hinzu, den die Ziel-2-Regionen erfassen dürfen (15\%), dann werden fast $42 \%$ der EG Bevölkerung in Fördergebieten leben.

Hervorzuheben ist noch die Festsetzung des Anteils der Fördergelder, die für Gemeinschaftsinitiativen reserviert sind. In der neuen Verordnung wurde dieser Anteil mit 5\% der Mittel festgelegt. Interessant ist, daß sowohl die Kommission als auch das Europäische Parlament eine Aufstockung dieses Anteils auf 15\% gefordert hatten, sich jedoch nicht gegen den Rat durchsetzen konnten. ${ }^{270}$

Die Reform der Strukturfonds zeigt zunächst interessante Parallelen zu der Entwicklung der GA auf. Auch auf europäischer Ebene sind Anpassungen bzw. Veränderungen von Zielen und Fördergebietsabgrenzungen nur pareto optimal möglich. Dies zeigt die Ausweitung der Fördergebiete und der Ziele sehr deutlich. Es gibt keine Ansätze für eine ordnungspolitische Regionalpolitik, die einen regelgebundenen Wettbewerb der regionalen Einheiten zulassen würde. Ansätze zur Dezentralisierung regionalpolitischer Zuständigkeiten finden sich nicht, vielmehr verstärkt die Reform tendenziell die Verflechtung und führt zu einer weiteren Zentralisierung. Diese Tendenz zeigt sich besonders deutlich bei der Diskussion über die Kontrolle der Verwendung von Strukturfondsmitteln. Das Problem wird nicht grundsätzlich gelöst. Anstatt Anreize für rent-seeking-Verhalten zu verringern und durch verstärkte Eigenverantwortlichkeit ${ }^{271}$ einen wirtschaftlicher Umgang mit den zur Verfügung stehenden Mitteln zu erreichen, fordert die Kommission eine stärkere Kontrolle. ${ }^{272}$

$\mathrm{Da}$ diese Tendenz auch in Zukunft zu erwarten ist, zeigt die Diskussion um die Quote für die Gemeinschaftsinitiativen. Kommission und Europäisches Parlament als Wahrer der europäischen Interessen verlangen mehr Kompetenz für die Ge-

Roland W. Waniek: "EG-Regionalpolitik furr die Jahre 1994 bis 1999", a.a.O., S. 44.

Vgl. Hartmut Hausmann: "Vom Mauerblümchen zum wichtigsten Finanzinstrument", a.a.O.

Die Stărkung der Eigenverantwortlichkeit und die Übertragung von vermehrten Entscheidungsbefugnissen steht bei jeder Verwaltungsreform im Zentrum des Prozesses. Statt durch immer mehr Kontrolle versucht man durch eine bewußte Dezentralisierung der Befugnisse die Wirtschaftlichkeit der Verwaltung zu erhohen. Diese Erkenntnis scheint sich jedoch in grőeren politischen Einheiten nur schwer durchzusetzen.

O.V.: "Fördermittel sollen besser kontrolliert werden - Kommissarin Wulf-Mathies reagiert auf Kritik an EU-Strukturfonds", FAZ vom 03.03.1995 / o.V.: "EU-Geld in neuen Ländern schlecht verwaltet", FAZ vom 04.11.1995. 
meinschaft. Sie wollen einen wesentlich größeren Teil der Gelder an europäischen Kriterien orientiert einsetzen und somit mehr Einfluß auf die nationalen Regierungen und Verwaltungen gewinnen. Sollten, was anzunehmen ist, die Zuständigkeiten des Europäischen Parlamentes mittelfristig erweitert werden, wird von ihm weiterer Druck in Richtung Zentralisierung ausgehen.

\subsubsection{Entwicklung der Regionalpolitik im Rahmen der GA}

Die Entwicklung der GA in den Jahren 1993 bis 1995 war durch folgende Faktoren gekennzeichnet.

1. Konflikt zwischen Bund/Ländern und der Kommission über die Fördergebietsabgrenzung ${ }^{273}$

2. Konflikt zwischen Bund, Ländern und der Kommission um die Ziele, Schwerpunkte und Förderinstrumente

3. Konflikt zwischen Bund und neuen Ländern, da nicht mehr alle Länder die gesamten EFRE Gelder im Rahmen der GA einsetzen wollen

4. Ausweitung der Fördertatbestände und mehr Entscheidungsspielraum für die Länder im Rahmen einer Reform der GA

Die Fähigkeit der GA, ihre Koordinierungsfunktion für die Regionalpolitik wahrzunehmen, wurde in den Jahren 1993 bis 1995 durch eine Vielzahl von Konflikten erheblich geschwächt. Zwischen dem Bund und der Kommission kam es zu Auseinandersetzungen um die Förderphilosophie der Bundesregierung. Die von der Bundesregierung für den Zeitraum 1994-99 vorgelegten Förderprogramme sahen die hundertprozentige Kopplung der EFRE Mittel an die GA vor. Wie in den Jahren 1991-93 sollten die EG-Mittel gemäß den restriktiven Vorgaben der GA auf die Förderung gewerblicher Investitionen und wirtschaftsnaher Infrastruktur konzentriert werden. Die Kommission, die eine wesentlich breitere Palette an Fördermöglichkeiten für sinnvoller hält, möchte die engen Bestimmungen der GA aufweichen. Sie ist sehr daran interessiert, Teile der EFRE-Mittel neben der GA direkt für Programme der Länder einzusetzen. ${ }^{274}$

Die neuen Länder vertraten unterschiedliche Positionen. MecklenburgVorpommern, Sachsen-Anhalt und Thüringen unterstützten Ende 1993 die Position des Bundes. Berlin, Sachsen und Brandenburg kündigten an, daß sie Teile der

Wie bereits mehrfach erwăhnt, kommt es aufgrund nicht identischer Indikatoren zu Unterschieden zwischen der Gemeinschaft und dem Bund in der Bestimmung der Fördergebiete. Dieses Problem war auch im Zeitraum 93-95 virulent.

Vgl. o.V.: "EU-Kommission erhebt Einwănde gegen Förderphilosophie der Bundesregierung", Handelsblatt vom 21.03.1994 / o.V.: "Rebellion im Osten", Die Zeit vom 13.05.1994. 
EFRE Gelder außerhalb der GA einsetzen wollen. Schwerpunkte für diese Programme sollten Umweltschutzmaßnahmen, grenzüberschreitende Zusammenarbeit und die Förderung von KMU sein. Für die neuen Länder ist die Herausnahme von EFRE Geldern aus der Bindung an die GA deshalb so interessant, weil damit Fördergelder für Maßnahmen bereitgestellt werden können, die im Rahmen der GA nicht förderfähig sind.

Der Bund war bemüht, die Kopplung der EFRE-Mittel an die GA aufrecht zu erhalten und die Konzentration der Mittel auf die gewerbliche Wirtschaft und wirtschaftsnahe Infrastruktur fortzusetzen. Seine Position gegenüber der Kommission war dadurch geschwächt, daß nicht nur drei Länder im Sinne der Kommission argumentierten, sondern auch der Bundesumweltminister eine Ausweitung der Fördertatbestände befürwortete, um Fördergelder für dringend benötigte Umweltschutzinvestitionen zu bekommen. ${ }^{275}$

Die Förderprogramme für die neuen Länder waren Ende 1993 der Kommission zugeleitet worden. Die oben beschriebene Konstellation führte dazu, daß im Juni 1994 eine Einigung zwischen Bund und Kommission erzielt wurde und die Förderprogramme im August von der Kommission gebilligt wurden ${ }^{276}$. Die Einigung ging allerdings zu Lasten des Bundes, da dieser einem Einsatz von EFRE Geldern außerhalb der GA zustimmen mußte. ${ }^{277}$

Dieser Prozeß zeigt beispielhaft, wie gering die Koordinationsfähigkeit der GA bezüglich der Förderprogramme, Förderschwerpunkte und der Gebietsabgrenzung noch ist. Die unterschiedlichen Interessen der beteiligten Akteure ließen sich nicht mehr im Sinne der GA zusammenfassen.

Als logische Reaktion auf diese Auseinandersetzung muß man die dann folgende grundlegende Reform der GA im Zuge der Aufstellung des 24. Rahmenplans sehen. Die Reform vereinfacht das Regelwerk und weitet die Fördertatbestände sowie die Fördersätze im Sinne der Länder erheblich aus. ${ }^{278}$ Zur Vereinfachung trägt bei, daß keine Differenzierung mehr der Förderhöchstsätze für die einzelnen Investitionsarten (Errichtung, Erweiterung, Erwerb, Rationalisierung) vorgesehen ist. Die GA löst sich von der reinen Investitionsförderung. Zukünftig sollen auch Planungs- und Beratungsleistungen für Kommunen bzw. für Unternehmen gefördert werden können. Viele der Veränderungen zielen auf eine stärkere Förderung

Vgl. o.V.: "Geldfluß von Brussel nach Ostdeutschland stockt", Frankfurter Rundschau vom 31.03.1994.

Vgl. o.V.: "Brüssel billigt Förderprogramme", Handelsblatt vom 09.08.1994.

Vgl. o.V.: "Streit um Fördermittel beigelegt", Handelsblatt vom 22.06.1995.

Die Veränderungen werden ausfuhrlich, im Rahmen einer synoptischen Darstellung, im Jahreswirtschaftsbericht 1995 des Landes Mecklenburg-Vorpommern dargestellt. 
des Mittelstandes ab. So wird die Förderung von Gewerbezentren für KMU zulässig - bisher waren nur Technologiezentren förderfähig - und es werden für innovative, exportorientierte oder strukturbestimmende Unternehmen Sonderförderungen eingeführt, die zusammen mit anderen Beihilfen bis zu $50 \%$ betragen können.

Die Ausweitung der Fördermöglichkeiten vergrößert den Handlungsspielraum der neuen Länder. So können jetzt im Rahmen der GA Förderprogramme realisiert werden, die bisher nur als Landesprogramme möglich waren. Ferner bietet die breitere Palette der Fördermöglichkeiten die Chance, die Förderprogramme stärker an den regionalen Präferenzen zu orientieren.

Die nachwievor praktizierte Mischfinanzierung der GA verhindert weiterhin die Einführung von ökonomischen Anreizen zum wirtschaftlichen Umgang mit den Fördergeldern. Auch die Berücksichtigung regionaler Präferenzen wird durch dieses Finanzierungssystem behindert. Es gibt keine Veränderungen, die auf eine ordnungspolitische Regionalpolitik und eine Dezentralisierung der regionalen Strukturpolitik ausgerichtet sind. Das System der Politikverflechtung, der Mischfinanzierung und der quantitativ interventionistische Politikansatz bleibt bestehen. Auch das Land Mecklenburg-Vorpommern geht von der zentralen Planung und Durchführung der Förderprogramme nicht ab. Die Effizienz und Effektivität der Regionalförderung wird mit den vorgenommenen Korrekturen nur geringfügig verbessert.

Auch hier zeigt sich, daß keine der beteiligten politischen Institutionen bereit ist, ihr vermeintlich vorhandenes Einfluß- und Steuerungspotential aufzugeben bzw. die Verantwortung für bestimmte Aufgaben der Regionalpolitik zu übernehmen. Diese Entwicklung ist insbesondere im Lichte der immer stärker werdenden Diskussion um die Verschwendung von Fördergeldern in den neuen Ländern und der zunehmenden Defizite der öffentlichen Hände bedenklich. Der Versuch, den Transformationsprozeß und die auch daraus resultierende Knappheit der öffentlichen Kassen im Rahmen von verflochtenen Politikstrukturen ohne klare $\mathrm{Zu}$ weisung von Verantwortlichkeiten mit interventionistischen Programmkonzeptionen zu lösen, wird wahrscheinlich nicht mit Erfolg gekrönt sein. Die theoretische Analyse und die derzeitige Entwicklung sprechen vielmehr für den Einsatz ordnungspolitischer Steuerungsinstrumente, die Anreize für einen wirtschaftlichen Umgang mit den vorhandenen Ressourcen bieten.

\subsection{Ursachen der Reformblockade}

Die Veränderungen - das Wort Reform wäre in diesem Zusammenhang nicht angebracht - im Bereich der GA und des EFRE lassen keine eindeutige Richtung erkennen. Das System scheint nicht in der Lage zu sein, einen Ausweg aus der "Politikverflechtungsfalle" zu finden. Eine Abkehr von dem konstruktivistischen 
wirtschaftspolitischen Ansatz ist nicht erkennbar, es gibt lediglich graduelle Veränderungen bei der Zuweisung von Kompetenzen. Die Grundstruktur der gemeinsamen Finanzierung und Planung der Politik bleibt erhalten. Keiner der Beteiligten scheint gewillt und in der Lage, eigene Kompetenzen abzugeben bzw. die alleinige Verantwortung für Teilbereiche der Regionalpolitik zu übernehmen.

Um den Ursachen für dieses Phänomen auf den Grund zu gehen, muß man sich zunächst das komplexe Beziehungsgeflecht dieses Systems in Erinnerung rufen. $\mathrm{Da}$ gibt es zunächst auf jeder Ebene eine eigenständige Bürokratie, die mit der Planung, Implementation und Evaluation der Regionalpolitik betraut ist. Jede Bürokratie ist der Legislative der jeweiligen Ebene verantwortlich. Die Parlamente der Länder und des Bundes bewilligen nicht nur die Budgets der Bürokratien, sondern sind auch für die Zuweisung von Kompetenzen zuständig. Auf der Gemeinschaftsebene sind die Beziehungen noch etwas komplizierter, da hier neben dem gewählten Parlament, das nur ein eingeschränktes Budgetrecht hat, der Rat das Budgetrecht und die Kompetenzzuweisung dominiert. Schlußendlich ist die Beziehung zwischen den Wählern und der Legislative bzw. der von einem Teil der Legislative getragenen Exekutive von Interesse. Regierung und Regierungsmehrheit im Parlament werden ihre Politik stets so ausrichten, daß ein Machterhalt, d.h. eine Wiederwahl möglich ist. Neben den Beziehungen innerhalb der einzelnen Ebenen des föderativen Systems gibt es Abhängigkeiten zwischen den verschiedenen Ebenen. Im Bereich der Bürokratien gibt es z.B. Weisungsbefugnisse zentraler Einheiten gegenüber dezentralen. Auch im Bereich der Legislative gibt es vertikale Beziehungen. Veränderungen der Kompetenzzuordnung können z.B. in der Regel nur mit Zustimmung aller betroffenen Ebenen erfolgen.

Einen Erklärungsansatz für die Reformblockade liefert die ökonomische Theorie der Bürokratie. Sie interpretiert die Beziehung zwischen Legislative und Bürokratie als ein Tauschverhältnis. Die Legislative fragt öffentliche Güter nach, die Bürokratie stellt dieser Nachfrage ihr Angebot öffentlicher Güter gegenüber.

Zentrale These der ökonomischen Theorie der Bürokratie ist die Budgetmaximierungshypothese. ${ }^{279}$ In der Zielfunktion eines Beamten sind nicht altruistische Motive, sondern sein Nutzen ausschlaggebend. Der Nutzen eines Beamten wird u.a. durch Gehalt, Reputation, Macht und Output seiner Behörde bestimmt. Diese Faktoren sind eng mit der verfügbaren Budgethöhe verbunden. Dies gilt für die Bürokratien jeder Ebene des föderalen Systems. Bezogen auf die Zuweisung von Kompetenzen bedeutet dies: Die Bürokratien der Länder, des Bundes und der

Begründer der Budgetmaximierungshypothese ist William A. Niskanen. Einen guten Überblick uber seine okonomische Theorie der Bürokratie bietet William A. Niskanen: "Bureaucracy and Representative Government", Chicago, 1997, ders., "Bureaucrats and Politicians", in Journal of Law and Economics Nr. 18, 1975, ders., "Bureaucracy: Servant or Master?", London, 1973. 
Gemeinschaft haben ein ausgeprägtes Interesse daran, ihre jeweiligen Kompetenzen zu erhalten bzw. auszubauen. Die optimale Dezentralisierung der Kompetenzen würde einen Verlust von Zuständigkeiten und eine Reduzierung der Budgets auf Bundes und Gemeinschaftsebene zur Folge haben. Macht und Einfluß der Kommission und des Wirtschaftsministeriums würden reduziert, Personal würde überflüssig und die Position bei Budgetverhandlungen wäre erheblich geschwächt. $\mathrm{Da}$ jede Ebene bestrebt ist, ihre Kompetenzen zu erhalten oder zu mehren, führt dies im Ergebnis zu einer immer weiteren Vertiefung der Verflechtung. Nur so bleibt jede Ebene an dem Entscheidungsprozeß beteiligt und ist in der Lage, ihre eigene Existenz gegenüber der Legislative zu legitimieren. Die Eigeninteressen der Verwaltungen sind daher eine wesentliche Ursache der Reformblockade. Im Bereich der Regionalpolitik wird dies z.B. darin deutlich, daß regionale Entwicklungsprogramme, die eigentlich in die Zuständigkeit der Landesministerien fallen müßten, vom Bundeswirtschaftsministerium und der Kommission geprüft bzw. genehmigt werden müssen.

Als nächstes stellt sich die Frage, ob und inwieweit die Parlamente oder genauer gesagt, der die Regierung stützende Teil der Parlamente und die Regierung ein Interesse an einer Veränderung der bestehenden Verflechtungsstrukturen haben. Auch hier ist davon auszugehen, daß der eigene Nutzen der gewichtigste Faktor in der Zielfunktion politischer Mandatsträger ist. Ziel ihrer politischen Arbeit ist die Stimmenmaximierung, d.h. der Erhalt des Mandates bzw. der Erhalt einer regierungsfähigen Mehrheit. Die Analyse muß sich daher auf die Frage konzentrieren, welche Interessen die politischen Mandatsträger an einer ordnungspolitischen Regionalpolitik und einer damit verbundenen Dezentralisierung der Maßnahmenentscheidungen und einer Zentralisierung der Regelsetzungskompetenz haben könnten. Macht, Einfluß und Reputation eines Mandatsträgers hängen ganz eng mit seinen Kompetenzen und dem zu verteilenden Budget zusammen. Nur der Politiker, der Entscheidungen, insbesondere Verteilungsentscheidungen, treffen bzw. beeinflussen kann, ist in der Lage, Politik gemäß den Präferenzen seiner Wählerschaft zu machen. Auf jeder Ebene des föderalen Systems sind die politischen Mandatsträger daran interessiert, ihre Entscheidungskompetenzen und ihre Budgetspielräume $\mathrm{zu}$ wahren bzw. zu mehren. Welche Ebene sich in diesem Wettbewerb durchsetzt, hängt u.a. von den verfassungsrechtlichen Rahmenbedingungen ab. So hat z.B. der Bund sehr schnell alle Bereiche der konkurrierenden Gesetzgebung mit eigenen Gesetzten ausgefüllt.

Im Bereich der Regionalpolitik zeigt sich eine Tendenz, die Entscheidungskompetenzen und Verteilungspotentiale zunehmend auf zentraler Ebene anzusiedeln. Hier spiegeln sich die Anziehungskräfte der zentralen Institutionen wieder. Es kommt jedoch nicht zu einer vollständigen Verlagerung der Kompetenzen. Typisch für ein föderales System scheint zu sein, daß im Ergebnis jede Ebene eine Rolle in dem Entscheidungs- und Verteilungsprozeß spielt. Diese verflochtene 
Struktur resultiert somit aus dem eigennutzorientierten Bestreben politischer Mandatsträger, Entscheidungskompetenzen und Verteilungsspielräume zu erhalten bzw. zu mehren.

Die zunehmende Verflechtung und die Tendenz zur Zentralisierung müßten eigentlich zu einer offenen Auseinandersetzung über die Kompetenzzuordnung führen. Dieses läßt sich in verschiedenen Politikbereichen auch beobachten. Die Kommunen haben versucht, gegen die Länder ihre Zuständigkeit für die Müllsammlung und Müllbeseitigung mit Klagen bis zum Bundesverfassungsgericht zu verteidigen. Die Länder haben sich im Ratifizierungsprozeß des MaastrichtVertrages erhebliche Mitwirkungsrechte in europapolitischen Fragen gesichert. Allerdings gibt es im Bereich der Regionalpolitik bisher nur ein Land, Bayern, das die Verlagerung der wesentlichen Entscheidungskompetenzen und Verteilungsspielräume auf die Landesebene fordert. ${ }^{280}$ Es muß also Bereiche geben, in denen es nicht im Interesse des politischen Mandatsträgers ist, alleine für eine Entscheidung verantwortlich $\mathrm{zu}$ sein. Um diese Bereiche herauszufiltern, muß man die Wirkung von Entscheidungskompetenzen und Verteilungspotentialen mit in Betracht ziehen.

Eine nicht geteilte Entscheidungskompetenz bedeutet, daß die Verantwortung für die Entscheidung und deren Folgen klar der zuständigen Ebene zuzuordnen ist. Politische Entscheidungen sind stets Entscheidungen unter Unsicherheit und der politische Mandatsträger weiß nie genau, ob seine Entscheidung den Präferenzen der Wähler entspricht. Er hat somit die Chance, mit im Sinne der Wählerpräferenzen richtigen Entscheidungen, seine Wiederwahl zu sichern, trägt jedoch auch das Risiko, durch falsche Entscheidungen sein Mandat zu verlieren. Nun gibt es Entscheidungen, bei denen eindeutig ist, ob sie positive oder negative Auswirkungen auf die Wähler haben und es gibt solche, die eindeutig einen ambivalenten Charakter haben, d.h. sie nutzen dem einen Teil der Wähler und schaden dem anderen. Der politische Mandatsträger ist daher bestrebt, immer dann die alleinige Entscheidungskompetenz zu haben, wenn die positiven Auswirkungen überwiegen. Bei ambivaleten oder mit sehr großer Unsicherheit behafteten Entscheidungen hat er ein großes Interesse, die Verantwortung auf mehrere Schultern zu verteilen. Gemeinsame Entscheidung bedeutet gemeinsame Verantwortung und im Zweifel kann die Schuld für eine Fehlentscheidung der jeweils anderen Ebene zugewiesen werden.

Nun sollte man annehmen, daß Verteilungspotentiale grundsätzlich etwas Positives für einen politischen Mandatsträger sind. Solange diese Potentiale groß genug

280 In einem Positionspapier vom 17.09.1996 fordert die Bayrische Staatsregierung eine Reform der Struktur- und Regionalpolitik. Sie verlangt eine Stärkung der regionalen Eigenverantwortung und fordert die EU auf, sich auf Rahmenvorgaben zu beschränken. Vgl. „Positionspapier der Bayrischen Staatsregierung fur eine Reform der Struktur- und Regionalpolitik der EU“, Munchen, 17.09.1996. 
sind, um die Ansprüche der Wähler zu befriedigen, ist dies auch zutreffend. Sobald die Verteilungspotentiale jedoch stagnieren bzw. schrumpfen oder gemessen an den an sie gestellten Ansprüche zu klein sind, wird aus der Lust eine Last. Die aus einer solchen Situation entstehenden Verteilungskämpfe fordern Entscheidungen, die im Zweifel mehr Menschen schaden als nutzen. Daher ist es in einer solchen Situation vorteilhaft, wenn die Verteilungsbudgets von verschiedenen Ebenen verantwortet werden. Die notwendigen Einschnitte können dann gemeinsam entschieden und gemeinsam verantwortet werden. Im Zweifel wird versucht, die negativen Entscheidungen bzw. die Schuld auf die jeweils andere Ebene zu verlagern.

Die oben skizzierten Beziehungen führen dazu, daß es im Rahmen der GA weder vor noch zurück geht und kaum eine Landesregierung massiv mehr Kompetenzen einfordert. Entscheidungen über Förderprogramme und Förderschwerpunkte sind im Zeichen knapper Verteilungsbudgets stets Entscheidungen, die einen nicht unerheblichen Teil der Wähler benachteiligen. Der Hinweis auf Bonner oder Brüsseler Vorgaben entlastet daher den politischen Mandatsträger auf Landesebene. Es hat auch noch keinen Landespolitiker gegeben, der massiv für die Abschaffung der zweckgebundenen Zuschüsse eintritt. Für den Bereich des Wirtschaftsministers würde dies bedeuten, daß statt 30\% 100\% der Mittel ohne Bindungen der Gemeinschaft oder des Bundes ausgegeben werden könnten. Die Verteilungskonflikte müßten somit im Land ausgetragen werden und sind nicht mehr a priorie durch die Bindung der Gelder gelöst. Der landespolitische Mandatsträger hat kein großes Interesse, diese verteilungspolitischen Entscheidungen alleine $\mathrm{zu}$ treffen und zu verantworten.

Für die politischen Mandatsträger der Bundes- und Europaebene bedeutet dies, daß sie stets ein Interesse daran haben, Entscheidungskompetenzen und Verfügung über Verteilungsbudgets zu haben. Jedoch haben sie kein Interesse daran, alleine zu entscheiden und zu verantworten. Das eigenutzorientierte Verhalten des auf Stimmenmaximierung ausgerichteten politischen Mandatsträgers tendiert da$\mathrm{zu}$, die vorhandenen ineffizienten Verflechtungsstrukturen $\mathrm{zu}$ verfestigen. Die derzeitige Reformblockade verhindert eine ordnungspolitische Regionalpolitik und die notwendige Dezentralisierung der Kompetenzen.

Bleibt die Frage zu klären, ob innerhalb der bestehenden politik-ökonomischen Rationalitäten auch in Zukunft die Umsetzung einer ordnungspolitischen Regionalpolitik unmöglich ist. Bisher haben die oben dargestellten Wirkungsmechanismen eine durchgreifende Reform der institutionellen Strukturen verhindert. Diese Blockade wird allerdings nur solange Bestand haben, wie sie im Interesse der handelnden Akteure ist, d.h. solange sie der Machtsicherung dient. In einer demokratischen Gesellschaft kann mittel- und langfristig nur die Politik Macht erhalten, die dem Bürger als für ihn nützlich bzw. vorteilhaft vermittelt werden kann. Im Zuge der konstruktivistischen Planungs- und Steuerungseuphorie der siebziger 
und achtziger Jahre sind den Bürgern unzählige Versprechungen gemacht worden, die im Vertrauen auf die Fähigkeit der Politik auch geglaubt wurden. Heute wird zunehmend deutlich, daß die Politik ihre Zusagen nicht einhalten kann. Das tatsächliche Steuerungspotential ist erheblich geringer als das für die Umsetzung der ambitionierten Programme notwendige. Diese Erkenntnis wird noch durch die Globalisierung ökonomischer und politischer Strukturen verstärkt. Mit nationalem politischen Handeln können globale Prozesse nicht gesteuert werden. Mehr denn je laufen in einem solchen Umfeld die konstruktivistischen Politikansätze ins Lehre. Dies alles verstärkt die Zweifel der Wähler an der Handlungs- und Steuerungsfähigkeit der Politik und es wird für die politischen Akteure daher zunehmend schwieriger, konstruktivistische Politikansätze dem Bürger positiv zu vermitteln, da die Wirkungslosigkeit oftmals zu offensichtlich ist. Das fehlende Vertrauen der Wähler in die Handlungsfähigkeit der Politik drückt sich unter anderem in der geringen Bereitschaft aus, politische Verantwortung zu übernehmen und sei es nur durch einen Urnengang.

Aus diesen Entwicklungen resultieren zwei Tendenzen. Zum einen begehren die regionalen Einheiten gegen die zentrale Bevormundung auf. Der Politiker, dem es gelingt, sich als Sachwalter der Region zu positionieren, hat gute Chancen regionale Abstimmungen zu gewinnen. Um seine Position zu stärken, wird es in seinem Interesse liegen, die Kompetenzen der Region zu erweitern. Zum anderen versuchen die zentralen Instanzen ihre Glaubwürdigkeit wiederherzustellen. Dies gelingt nur dann, wenn die behaupteten Steuerungspotentiale den tatsächlichen entsprechen. Dies könnte erreicht werden, indem auf konstruktivistische Politikansätze verzichtet wird und Macht und Einfluß der zentralen Instanz in ordnungspolitischen Programmen manifestiert wird.

Die Tendenz zur Regionalisierung ist in vielen Ländern Europas zu beobachten. Die regionalen politischen Akteure haben ein großes Interesse an diesen Prozessen, da sie ihnen einen erheblichen Zugewinn an Kompetenzen und Macht bringen. Unter der Annahme des nutzenmaximierenden regionalen Politikers ist es daher durchaus plausibel, wenn auch die Regionalisierung der Regionalpolitik gefordert wird. In der Tendenz geschieht dies in Bayern, aber auch in Italien hat man erkannt, daß nur ein eigenverantwortlicher Süden, der frei ist von zentraler Bevormundung, die Chance hat, sich politisch und wirtschaftlich zu entwickeln. ${ }^{281}$ Schwieriger sind die Prozesse auf der zentralen Ebene. Hier ist zunächst ein Verlust von Steuerungspotential im konstruktivistischen Sinne zu gegenwärtigen. Daher sind auf dieser Ebene die Widerstände auch besonders groß. Doch auch hier

Die FAZ berichtet in ihrer Ausgabe vom 03.01.97 unter der Überschrift „Keine Entwicklungspolitik ist die beste für den Mezzogiorno" von den Erkenntnissen italienischer Fachleute und Politiker uber die bisher praktizierte „planwirtschaftliche Entwicklungspolitik“. Die Reformvorschlăge bedeuten, so die FAZ, ,... den Abschied von jeder Programmpolitik, die vorgibt, Entwicklung fordern zu wollen und zu können.“ 
wird zunehmend deutlich, daß ein Beharren auf hergebrachten Konzepten zu noch größeren Verlusten von Einfluß und Macht führt. Die Eigennutzorientierung könnte ein Umdenken zu neuen, ordnungspolitischen Steuerungsinstrumenten befördern. Auf der nationalen Ebene ist dieser Mechanismus mit einer gewissen Wahrscheinlichkeit möglich. Auf der supranationalen Ebene ist dieser Schritt eher unwahrscheinlich, da man hier unverändert glaubt, gestützt auf die Größe der Struktur, konstruktivistische Politikansätze vertreten zu können. Erschwerend kommt hinzu, daß die Gemeinschaft ihre Kompetenzen von den Mitgliedsstaaten zugewiesen bekommt. Um Macht und Einfluß zu festigen und Institutionen zu legitimieren, überwiegt bei den europäischen Institutionen der Wunsch nach mehr Kompetenzen. Ob die Herren der europäischen Verträge, die Mitgliedstaaten, die Größe haben, die Gemeinschaft mit der für eine ordnungspolitische Regionalpolitik notwendigen Regelsetzungskompetenz auszustatten, bleibt fraglich. Ohne eine verfassungsmäßige Verankerung der Regelsetzungskompetenz auf europäischer Ebene und der bewußten Beschränkung der Gemeinschaft auf diese Funktion, ist die Einführung einer ordnungspolitischen Regionalpolitik nicht erwartbar. Ausgehend von den Mitgliedstaaten müssen daher die institutionellen Strukturen, d.h. die europäischen Verträge so geändert werden, daß die politik-ökonomischen Rationalitäten die Einführung einer ordnungspolitischen Regionalpolitik unterstützen.

\subsection{Zusammenfassung}

Um eine ordnungspolitische Regionalpolitik zu realisieren, müssen institutionelle Reformen durchgeführt werden, die sich an zwei grundsätzlichen Prinzipien orientieren. Zum einen muß es eine zentrale Institution geben, die die Funktion der Regelsetzung und Regeldurchsetzung wahrnimmt, damit eine Wettbewerbsordnung für einen Wettbewerb der regionalen Einheiten entstehen kann. Die konkreten Mittel-Zweck Entscheidungen, also die Entwicklung und Implementation von Programmen, ist unter diesen Bedingungen so weit wie möglich zu dezentralisieren. Diese grundlegende Zuordnung der Kompetenzen muß durch eine entsprechende Ausgestaltung der Finanzverfassung abgestützt werden. Die Entscheidungskompetenzen der regionalen Einheiten müssen durch adäquate Finanzierungskompetenzen abgesichert sein. $\mathrm{Ob}$ dies durch eine Zuweisung von ungebundenen Transfers nach dem Prinzip der fiskalischen Gleichheit oder durch die Stärkung eigener Steuererhebungsrechte der regionalen Einheiten geschieht, ist in der Literatur umstritten. Entscheidend ist, daß die regionalen Einheiten unabhängig von zentralen Einflüssen über ihre Budgetverwendung entscheiden können müssen.

Für die Reform der Regionalpolitik bedeutet dies, daß die Beihilfenkontrolle der Gemeinschaft im Sinne einer allgemeinen Regelsetzung ausgebaut werden muß. Die Kommission hätte gemäß den Verträgen hierzu potentiell die Möglichkeit. Sie muß jedoch gleichzeitig auf jede ziel- und zweckgerichtete Regionalförderung 
verzichten, d.h. die Strukturfonds und der Kohäsionsfonds müßten aufgelöst werden. Die GA hätte in einem solchen System keine Legitimation mehr. Die Funktion der Regelsetzung übernimmt die Gemeinschaft und Platz für eine leitbildorientierte Regionalpolitik auf Bundesebene ist in einer ordnungspolitischen Regionalpolitik nicht. Für die Gemeinschaft wie für die Bundesrepublik gilt, daß dieses System durch eine Reform der Finanzverfassung abgestützt werden muß, da nur so die Anreizstrukturen entstehen, die im Sinne der regionalen Präferenzen eine effiziente Allokation der Mittel gewährleisten.

Die tatsächlichen Veränderungen im Bereich der GA und der Gemeinschaft tendieren jedoch in eine andere Richtung. Im Ergebnis werden die bestehenden Verflechtungsstrukturen verfestigt und keine Elemente einer ordnungspolitischen Regionalpolitik eingeführt. Die europäische Regionalpolitik dominiert zunehmend die GA und zeigt die für verflochtene Systeme typischen Entwicklungstendenzen.

Eine Ursache für die Reformblockade und die Verfestigung der „Politikverflechtung" ist die Tendenz zur Budgetmaximierung, die durch die ökonomische Theorie der Bürokratie begründet wird. Auf jeder Ebene des verflochtenen Systems EG wird Macht und Einfluß der Bürokratien maßgeblich durch die Budgethöhe bestimmt. Daher liegt es im bürokratischen Interesse, einen Teil der Entscheidungs- und Finanzierungskompetenz zu haben, um die eigene Existenz legitimieren zu können. Eine weitere Ursache findet sich in der Eigenutzorientierung der politischen Mandatsträger. Die Regierungen wie die sie tragenden Parlamentsmehrheiten brauchen zum Machterhalt Verfügungsgewalt über Verteilungsbudgets, die sie im Sinne der Wählerpräferenzen einsetzen können. Daher ist keine Ebene gewillt, endgültig auf Verteilungskompetenzen zu verzichten, was dann letztendlich zu einer Festschreibung der Politikverflechtung führt.

Diese politik-ökonomische Rationalität kann nur dann aufgebrochen werden, wenn die Nachteile dieses Systems dem Wähler offenbar werden und somit den Machterhalt der Mandatsträger gefährden. Das insbesondere im Zuge der Globalisierung immer deutlicher werdende Versagen konstruktivistischer Politikansätze könnte eine Reform in Richtung ordnungspolitischer Regionalpolitik ermöglichen. Immer mehr Regionen setzen sich gegen die zentrale Bevormundung zur Wehr und fordern mehr regionalpolitische Kompetenzen. Diese Entwicklung wird von regionalen Mandatsträgern unterstützt, da sie Macht und Einfluß stärken kann. Jeder Politiker, der sich als Sachwalter regionaler Interessen positionieren kann, hat gute Chancen auf Unterstützung durch die Wähler. Die Mandatsträger der zentralen Ebene haben wenig Interesse an diesen Prozessen, da sie zugunsten der Regelsetzungskompetenz auf Verteilungsentscheidungen verzichten müssen. Auf der nationalen Ebene ist dieser Prozeß noch vorstellbar, da die Unzulänglichkeit konstruktivistischer Politikansätze zu offensichtlich ist. Die Gemeinschaft, als 
junge institutionelle Ebene, tendiert jedoch nach wie vor zur Ausweitung ihrer Kompetenzen. Ferner scheint man hier zu glauben, daß aufgrund der Größe konstruktivistische Politikansätze Aussicht auf eine erfolgreiche Steuerung wirtschaftlicher Prozesse haben.

Eine ordnungspolitische Regionalpolitik ist daher nur dann möglich, wenn die Kompetenzen der Gemeinschaft in den europäischen Verträgen auf die Regelsetzung und Regeldurchsetzung beschränkt werden. Ob die Mitgliedstaaten als Herren der Verträge hierzu in der Lage sind, bleibt mehr als fraglich. 


\section{Zusammenfassung der Arbeit}

Institutionen bestimmen die Anpassungsfähigkeit und die Innovationsfähigkeit politischer und wirtschaftlicher Systeme. Diese Fähigkeiten determinieren in einer Zeit der wahrscheinlich epochalen Wandlungen das Wachstums- und Leistungspotential einer Volkswirtschaft. Zu Beginn des fünften Kondratief gilt es, die politischen und wirtschaftlichen Strukturen an die bereits bekannten bzw. absehbaren Herausforderungen der Globalisierung und des Informationszeitalters anzupassen. Die Arbeit hat die Innovations- und Anpassungsfähigkeit in Deutschland und Europa am Beispiel der Regionalpolitik untersucht. Regionalpolitik ist „... die Summe aller Bestrebungen, Maßnahmen und Handlungen, welche die Stimulierung und Lenkung von Entwicklungen in der räumlichen Verteilung ökonomischer Aktivitäten verfolgen. ${ }^{\prime 282}$ Legte man der Arbeit diese Definition zugrunde, gebe es kaum einen Politikbereich, der nicht betrachtet werden müßte. Wegen der hohen politischen und finanziellen Bedeutung wurde im Rahmen dieser Untersuchung nur der Teil der Regionalpolitik betrachtet, der im Rahmen des EFRE, der GA und vergleichbarer Landesprogramme durchgeführt wird.

Die Analyse der Regionalpolitik sowie der sie beeinflussenden institutionellen Strukturen fußt auf folgenden theoretischen Überlegungen:

1. Das wirtschaftspolitische Konzept, das einem regionalpolitisches Programm zugrundeliegt, ist ein wichtiges Merkmal zur Differenzierung und Bewertung von Förderprogrammen. Kriterium für die Differenzierung wirtschaftspolitischer Konzepte sind die explizit oder implizit formulierten Annahmen zur Plan- und Steuerbarkeit wirtschaftlicher Prozesse. Man kann zwischen dem Konzept Marktversagen und Planbarkeit, Politikversagen und einem ordnungspolitischen Konzept unterscheiden. Das erste Konzept unterstellt, daß die unzureichenden Allokationsergebnisse der Marktkräfte durch die bewußte Planung und Steuerung der wirtschaftspolitischen Akteure korrigiert werden müssen. Für die Vertreter des zweiten Konzeptes sind es vielmehr die politisch gesetzten Institutionen, die die Marktkräfte behindern und somit die eigentliche Ursache für Fehlallokationen darstellen. Das ordnungspolitische Konzept geht grundsätzlich von der Funktionsfähigkeit der Märkte aus. Es ist dem Staat nicht möglich, wirtschaftliche Prozesse bewußt zu planen. Seine primäre Aufgabe besteht in der Setzung und Durchsetzung von allgemeinverbindlichen Regeln.

2. Desweiteren müssen die regionalwirtschaftlichen Theorien betrachtet werden, die Basis der Förderprogramme sind. Neben der Theorie der zentralen

Harald Jürgensen: „Raumwirtschaft II: Politik“, in Handwörterbuch der Wirtschaftswissenschaft, 1980, S. 429. 
Orte sind dies die Exportbasistheorie, die Theorie der Wachstumspole und Elemente der neuen Wachstumsmodelle. Die regionalwirtschaftlichen Theorien, die regionalpolitisches Handeln allokations- bzw. distributionspolitisch begründen, basieren auf einem konstruktivistischen wirtschaftspolitischem Konzept. Da dem politischen Planer nie alle relevanten Informationen, die zur Anwendung der Theorien notwendig sind, zur Verfügung stehen, werden regionalpolitische Entscheidungen durch die politische Rationalität dominiert.

3. Die institutionelle Analyse, die sich auf die Frage der Kompetenzzuweisung im föderalen System konzentriert, basiert auf dem Konzept des fiscal federalism und dem dynamischen Wettbewerbskonzept von Hayeks. Mit dem Konzept der fiskalischen Äquivalenz unter Berücksichtigung der regionalen Präferenzen läßt sich die bestehende Struktur des föderalen Systems analysieren. Für die notwendige dynamische Betrachtung greift die Arbeit auf das dynamische Wettbewerbskonzept Hayeks zurück. Indem dieses auf die Beziehungen zwischen regionalen Einheiten angewandt wird, ergibt sich ein ordnungspolitisches Konzept der Regionalpolitik, das im weiteren Verlauf der Arbeit Maßstab für die Analyse regionalpolitischer Programme ist.

Folgende Thesen galt es am Beispiel der Regionalpolitik in MecklenburgVorpommern zu prüfen:

a. Die deutsche und europäische Regionalpolitik ist durch konstruktivistische wirtschaftspolitische Konzeptionen geprägt. Hierin liegt ihr primäres Defizit und die Ursache für die geringe Effektivität.

b. Die aus den bestehenden institutionellen Rahmenbedingungen resultierende Kompetenzzuweisung führt - im Sinne der regionalen Präferenzen und der fiskalischen Äquivalenz - zu einer ineffizieten Allokation regionalpolitischer Mittel.

c. Eine Reform muß sich an dem Leitbild einer ordnungspolitischen Regionalpolitik orientieren. Hierfür sind grundlegende Änderungen der institutionellen Strukturen notwendig.

d. Die vorhandenen politik-ökonomischen Rationalitäten stehen einer durchgreifenden Reform der institutionellen Strukturen im Wege. Es sind lediglich partielle Veränderungen realisierbar.

Die empirische Analyse orientiert sich an dem idealtypischen Ablauf eines politischen Planungsprozesses. Basierend auf einer Lageanalyse, die Stärken und Schwächen herausarbeitet, werden Ziele definiert, Instrumente entwickelt, implementiert und evaluiert. Die Ergebnisse dieses Prozesses sind dann Ausgangspunkt für einen erneuten Durchlauf des Planungsprozesses. 
Mecklenburg-Vorpommern steht vor enormen wirtschaftspolitischen Herausforderungen, da es hinsichtlich der Wirtschaftsstruktur, der Bevölkerungsdichte und verteilung sowie auf Grund seiner Lage erheblich Schwächen hat. Regionalpolitik in Mecklenburg-Vorpommern wird im Rahmen eines Systems geplant und implementiert, das durch Mischfinanzierung und Politikverflechtung gekennzeichnet ist. In allen Phasen des Planungsprozesse sind die EG, der Bund, das Land sowie teilweise regionale Einheiten beteiligt. Auf der Ebene der Zielsetzung zeigen sich bereits erhebliche Defizite des Systems:

- Da die Zielsetzung in einer verflochtenen Struktur durchgeführt wird, binden die Ziele der zentralen Ebene die dezentrale. Damit werden die Handlungsfreiräume der regionalen Einheiten bereits in dieser Phase der Planungsprozesses eingeschränkt.

- Die Ziele lassen eine selektive Orientierung an bestimmten Regionaltheorien erkennen. Es gelingt allerdings nicht, ein widerspruchsfreies und hinreichend operationalisiertes Zielsystem zu definieren.

- Die Analyse der Zielsetzung zeigt, daß die Regionalpolitik auf konstruktivistischen Politikkonzeptionen beruht und eine Ausrichtung an einem ordnungspolitischen Konzept der Regionalpolitik nicht erkennbar ist.

Bei der Umsetzung der Ziele in regionalpolitische Programme werden die Defizite noch deutlicher. Mischfinanzierung und Politikverflechtung, Mißachtung der fiskalischen Äquivalenz und der regionalen Präferenzen führen zu rent-seekingVerhalten und einer ineffizienten Allokation knapper Mittel. Das System reagiert auf diese Defizite mit einer ausufernden Kontrollbürokratie und einer Vertiefung der Verflechtung. Die Programme orientieren sich an unterschiedlichen Regionaltheorien. Hierbei wird die sehr begrenzte praktische Umsetzbarkeit dieser Modelle deutlich. Alle Programme basieren auf konstruktivistischen Politikkonzeptionen. Freiräume für eine ordnungspolitische Regionalpolitik sind nicht erkennbar.

Zielvorgabe für die Implementation der regionalpolitischen Programme ist das Ausgleichsziel. Förderbedürftige Regionen sollen stärker gefördert werden als potentiell stärkere Regionen. Es gelingt dem Land Mecklenburg-Vorpommern nicht, die Mittel entsprechend dieser Zielvorgabe einzusetzen. Die statistische Analyse läßt keinen Zusammenhang zwischen Förderbedürftigkeit und Förderintensität erkennen. Der Anspruch der konstruktivistischen Programme läßt sich in der Realität nicht durchhalten, die Implementation orientiert sich an anderen als den in der Planung vorgegebenen Zielen.

Die Ursachen für diesen Befund sind vielfältig. Neben unberücksichtigten Zielkonflikten spielen u.a. die unzureichende theoretische Fundierung, die mangelnde Operationalisierung und ein ungesteuerter Förderwettbewerb zwischen den Län- 
dern eine Rolle. Entscheidend für die Defizite und die Stabilität des verflochtenen Systems sind jedoch politik-ökonomische Gründe. Unter der Annahme des nutzenmaximierenden Verhaltens ist das Ergebnis aus Sicht der Entscheidungsträger in Wirtschaft und Politik rational. Zunächst dienen die Regionaltheorien zur ökonomischen Legitimierung regionalpolitischer Aktivitäten. Die Umsetzung folgt jedoch nicht ökonomischen, sondern politik-ökonomischen Erwägungen, da die Verteilung der Gelder im Sinne der Nutzenmaximierung zur Sicherung von Macht eingesetzt wird.

Im Konzept einer ordnungspolitischen Regionalpolitik kommt den Ländern eine besondere Bedeutung zu, da auf dieser Ebene konkrete regelgebundene MittelZweck-Entscheidungen gefällt werden. Im bestehenden System werden die Handlungsspielräume der Länder durch die Vorgaben der GA und der Gemeinschaft eingeschränkt. Grundsätzlich sind die Vorgaben der GA für die Länder bindend, da der Bund jedoch keine Handhabe hat, diese durchzusetzen, kann die GA nicht die Funktion der Setzung und Durchsetzung von Regeln übernehmen. Die Beihilfenkontrolle der EG könnte grundsätzlich eine Basis für allgemeinverbindliche Regeln sein. Beihilfen sind mit dem gemeinsamen Markt nicht vereinbar und somit grundsätzlich verboten, es sein denn, sie sind für den Ausgleich bestimmter Problemlagen notwendig. Allerdings setzt ein gemeinsamer Markt auch Chancengleicheit voraus, zu deren Herstellung Beihilfen nicht nur erlaubt, sondern aus Sicht der Gemeinschaft geboten sind. Da die Gemeinschaft eigene regionalpolitische Ziele verfolgt, die u.a. mit der Beihilfenkontrolle erreicht werden sollen, schränkt diese die Freiräume der Länder zunehmend ein, kann jedoch nicht als Quelle allgemeinverbindlicher Regeln dienen.

Die Analyse der unter diesen Rahmenbedingungen bestehenden Landesprogramme zeigt sehr deutlich, daß das Land Mecklenburg-Vorpommern neben der GA und dem EFRE eigene regionalpolitische Schwerpunkte setzt, die sich gezielt an den landesspezifischen Problemen orientieren. In dem bestehenden System kommt diesen Programmen wegen der starken Beschränkungen und dem geringen finanziellen Volumen allerdings nur eine untergeordnete Bedeutung $\mathrm{zu}$.

Nach dem Beobachtungszeitraum wurden ab 1994 Reformen der GA und des EFRE durchgeführt. Resultat dieser Reformen sind Veränderungen im bestehenden System. Die grundlegenden Mängel - konstruktivistischer Politikansatz, keine ordnungspolitische Regionalpolitik - bleiben erhalten. Eine an dem Leitbild der ordnungspolitischen Regionalpolitik orientierte Reform müßte folgende Merkmale aufweisen:

1. Verzicht auf konstruktivistische Politikansätze auf Ebene der EG. Einstellung der Regionalpolitik über die Regionalfonds, Konzentration auf die Setzung und Durchsetzung eines allgemeinverbindlichen Ordnungsrahmens für 
die Regionalpolitik gemäß Art 92 ff EWGV. Redistributive Politik kann wesentlich effizienter über eine entsprechende Ausgestaltung einer europäischen Finanzverfassung erfolgen.

2. Auf Bundesebene Abschaffung der GA, Dezentralisierung der Planung und Durchführung regionalpolitischer Programme.

3. Diese Schritte müssen ergänzt werden, um eine adäquate Ausgestaltung einer europäischen bzw. bundesdeutschen Finanzverfassung.

Die Aussicht auf die Umsetzung einer ordnungspolitischen Regionalpolitik ist sehr gering. Die Tendenz zur Budgetmaximierung führt auf allen beteiligten Ebenen zur Verfestigung der bestehenden Strukturen. Die Eigennutzorientierung der politischen Entscheidungsträger macht einen Verzicht auf finanzielle Verteilungsspielräume sehr schwer, da nur diese eine Mittelallokation im Sinne der Wählerpräferenzen zur Machterhaltung ermöglichen.

Es sind allerdings auch im Rahmen der bestehenden politik-ökonomischen Rationalitäten Situationen denkbar, die die Einführung einer ordnungspolitischen Regionalpolitik unterstützen könnten. Dem Bürger wird das Versagen zentraler konstruktivistischer Politikansätze immer deutlicher. Die Regionen wehren sich zunehmend gegen zentrale Bevormundung und fordern mehr Kompetenzen und Entscheidungsfreiheit. Für einen regionalen Mandatsträger könnte es im Sinne des Machterhaltes sehr lohnend sein, diese Tendenzen aufzugreifen und zu verstärken. Jeder Politiker, der sich als Sachwalter der regionalen Interessen positionieren kann, hat gute Chancen auf Unterstützung durch die Wähler. Auf der überregionalen Ebene ist die Situation weniger eindeutig. Im Lichte der immer deutlicher werdenden Steuerungsdefizite könnte es auf dieser Ebene im Sinne der politischen Entscheidungsträger sein, einen Politikwechsel hin zu ordnungspolitischen Konzeptionen vorzunehmen. Sie verlieren zwar Verteilungspotentiale und vermeintliche Steuerungspotentiale, gewinnen jedoch die Regelsetzungs- und Regeldurchsetzungskompetenz. Für die Bundesebene ist diese Entwicklung noch vorstellbar, jedoch dominieren in der Gemeinschaft nachwievor die konstruktivistischen Politikkonzeptionen. Ferner müßten die Herren der Verträge, die Mitgliedstaaten, bereit und in der Lage sein, der Gemeinschaft diese Funktion zuzuweisen. Dies scheint bei den sehr unterschiedlichen politischen, historischen und wirtschaftlichen Rahmenbedingungen in den Mitgliedstaaten derzeit eher unwahrscheinlich. Unklar ist, wie die europäischen Nationen auf den immer stärkeren internationalen politischen und wirtschaftlichen Druck reagieren. Die Globalisierung der Märkte zwingt Europa, bestimmte politische und ökonomische Aufgaben zentral zu lösen. Dies gilt u.a. für die gemeinsame Währung, eine gemeinsame Außen- und Sicherheitspolitik und eine gemeinsame Verteidigungspolitik. Auf der anderen Seite suchen die Menschen in Europa nach regionalen Einheiten, die sie politisch und wirtschaftlich gestalten können, sie verlangen nach Entscheidungs- und Finanzierungskompetenzen und reagieren zunehmend ablehnend auf jede weitere Kompe- 
tenzübertragung nach Brüssel. Wenn ein Europa der ,25“ funktionieren soll, muß die Frage gestellt werden, wieviel und in welchen Bereichen dieses Europa Einheitlichkeit braucht. Bei allen wirtschaftspolitischen Fragen muß diese Antwort auf ordnungspolitischen Grundsätzen beruhen und im Zweifel dem Prinzip Wettbewerb den Vorzug lassen. Der äußere Druck zwingt Europa zum Handeln. Um seine Position halten zu können, muß es in wichtigen Dingen einheitlich agieren. Auf der anderen Seite muß es ein Höchstmaß an Freiheit und Wettbewerb ermöglichen, um genug Dynamik und Innovationen zu erzeugen als Basis für ein erfolgreiches Bestehen im globalen Wettbewerb. Wenn der ökonomische und politische Druck eine kritische Größe erreicht, ist es nicht ausgeschlossen, daß die notwendigen institutionellen Reformen umgesetzt werden. 


\section{Literaturverzeichnis}

\section{Monographien}

Braun, G. / Gerlach, D.

Buchanan, J.M. / Brennan, G

Domschke, W. / Drexl, A.

Dt. Spark.-u. Giroverband

Eckey, Hans-Friedrich

Eucken, Walter

Färber, Gisela

Fischer / Hax / Schneider

Grabitz / Hilf

Groeben, von der, et alia

Hayek, F.A., von

Hayek, F.A., von

Hayek, F.A., von

Hayek, F.A., von

Hayek, F.A., von
„Probleme der Regional- und Strukturpolitik in Mecklenburg-Vorpommern", Rostock 1994.

„The Power to Tax. Analytical Foundations of a Fiscal Constitution", Cambridge University Press, 1980.

„Logistik: Standorte“, München 1990.

„Ostdeutsche Wirtschaft im Wandel: Bestandsaufnahme und Perspektiven eines Aufholungsprozesses“, Stuttgart 1992.

„Grundlagen der regionalen Strukturpolitik“, Bund Verlag 1978.

„Grundsätze der Wirtschaftspolitik“, Tübingen 1952.

„Binnenmarktgerechte Subventionspolitik in der Europäischen Union“, Campus Verlag, 1995.

„Treuhandanstalt. Das Unmögliche wagen“, Berlin 1993

„Kommentar zur Europäischen Union“, C.H. Beck, München 1992.

„Kommentar zum EWG-Vertrag“, 1983.

„Individualismus und wirtschaftliche Ordnung“, Salzburg 1976.

„New Studies in Philosophy, Politics, Economics and History of Ideas“, London 1978.

„Recht, Gesetzgebung und Freiheit, Band 1: Regeln und Ordnung“, verlag moderne industrie, 1980.

„Recht, Gesetzgebung und Freiheit, Band 3: Die Verfassung einer Gesellschaft freier Menschen“, verlag moderne industrie, 1980.

„The constitution of Liberty“, London 1960. 
Hembach, Klaus

Ifo Institut / IWH

ifo Institut

IWH

Jones, E.L.

Klemmer, Paul et alia

Klemmer, Paul

Klodt, H / Paqué, K.-H.

Krieger-Boden, $\mathrm{Ch}$.

Krugmann, $\mathrm{P}$.

Lammers, Konrad

Neupert, Helmut

Niskanen, William A.
„Der Stellenwert von Wirkungsanalysen für die Regionalpolitik - Eine Systematisierung der Problematik am Beispiel der regionalen Wirtschaftspolitik“, Frankfurt a.M. 1980.

„Finanzierungsprobleme kleiner und mittlerer Unternehmen der gewerblichen Wirtschaft in den neuen Bundesländern“, München/Halle 1994.

„Die Effizienz der finanzpolitischen Fördermaßnahmen in den neuen Bundesländern", München 1994

„Wirtschaft im Systemschock“, Berlin 1994.

„The European Miracle“, Cambridge, 1981.

„Übertragung regionalpolitischer Konzepte auf Ostdeutschland", Untersuchung des RWI, Essen, 1991.

„Regionalpolitik auf dem Prüfstand“, Köln 1986.

„Am Tiefpunkt der Transformationskrise: Industrie- und lohnpolitische Weichenstellungen in den jungen Bundesländern", Kieler Diskussionsbeiträge, 1993

„Neue Argumente für Regionalpolitik? Zur Fundierung der Regionalpolitik in älteren und neueren regionalökonomischen Theorien", in: Die Weltwirtschaft, Heft 2/1995, S. 193 ff.

"History and industry location: The case of the US manufactoring belt" in: The American Economic Review, Vol. 81, 1991, S. 80 ff.

„Wege der Wirtschaftsförderung für die neuen Bundesländer", in: Die Weltwirtschaft, 1990, Heft 2, S. $106 \mathrm{ff}$.

„Regionale Strukturpolitik als Aufgabe der Länder: Grundlagen, Verknüpfungen, Grenzen", Baden-Baden 1986.

„Bureaucracy and Representative Government", Chicago 1997. 
Niskanen, William A.

Niskanen, Wliiam A.

North, D.C.

Nowak, Ralf

Oswald, Bernhard

Padoa-Schioppa, T.

Popper, Karl R.

Richardson, H.W.

Röpke, Wilhelm

Scharpf / Reissert / Schnabel

Seibel, Wolfgang

Siebert, Horst

Spiekermann, B.

Streintz, R.

Suntum, Ulrich, van

Tocqueville, A., de
„Bureaucracy: Servant or Master?“, London 1973.

„Bureaucrats and Politicians“, in: Journal of Law and Economics Nr. 18, 1975.

„Institutions, institutional Change and economic performance", 1990.

„Gesamtwirtschaftliche Aspekte von Existenzgründungshilfen des Bundes und der Länder“" Köln 1991.

„Erfolgskontrolle in der Regionalpolitik“, Vandenhoeck Ruprecht, 1980.

„Effizienz, Stabilität und Verteilungsgerechtigkeit“, Gabler 1987.

„Das Elend des Historizismus“, Tübingen 1971.

„Regional Growth Theory“, London 1973.

„Die Lehre von der Wirtschaft“, 8. durchgesehene Auflage, 1958.

„Politikverflechtung: Theorie und Empirie des kooperativen Föderalismus in der Bundesrepublik“, Kronberg 1976.

„Verwaltungsreform und Verwaltungspolitik im Prozeß der deutschen Einigung", BadenBaden 1993.

„Zur Theorie regionalen Wirtschaftswachstums“, Dissertation, Münster 1995.

„Europäische Regionalpolitik“, Köln 1988.

„Europarecht“, Heidelberg 1992.

„Regionalpolitik in der Marktwirtschaft“, Nomos 1981.

„Über die Demokratie in Amerika“ (1835), aus dem französischen von Hans Zbinden, Zürich 1987, Bd1. 
Wagener, H.J.

Wegner, Eckhard
„Anpassung durch Wandel - Evolution und Transformation von Wirtschaftssystemen", Schriften des Vereins für Socialpolitik, Berlin 1992.

„Finanzausgleich im vereinten Deutschland“, Metropolis, 1992.

\section{Artikel / Studien / Berichte}

Bayrische Staatsregierung

Berg, Hartmut

BMWi Außenstelle Berlin

Boss, A. / Trapp, P.

Boss, Alfred

Böventer, Edwin, von

Breul, Necker, Späth et alia

Buchanan, J.

Czada, Roland

Drexl, A.
„Positionspapier der Bayrischen Staatsregierung für eine Reform der Struktur- und Regionalpolitik der EU“, München, 17.09.1996.

„Wettbewerbspolitik“ in: Vahlens Kompendium der Wirtschaftstheorie und Wirtschaftspolitik, München 1990, S. 235-291.

„Konzept der Bundesregierung zur Sicherung und Erneuerung industrieller Kerne durch die Treuhandanstalt in den neuen Bundesländern", Berlin 1993.

„Sozialhilfe ist Sache der Gemeinden“, FAZ vom 30.05.1995.

„Für eine kommunale Einkommenssteuer“, FAZ vom 16.12.1994.

„Raumwirtschaft I: Theorie“ in: Handwörterbuch der Wirtschaftswissenschaft, Göttingen 1980, S. 407-429.

Zeitgespräch im Wirtschaftsdienst zum Thema: „Erhaltung industrieller Kerne in Ostdeutschland", WD 1993/II, S. 59 ff.

„Federalism and Fiscal Equity“, American Economic Review, Vol 40.

„Die Treuhandanstalt im politischen System der Bundesrepublik", in: Aus Politik und Zeitgeschichte, B43-44/94, vom 28.10.1994, S. 31-42.

„Standorttheorien“ in: Enzyklopädie der Betriebswirtschaftslehre, Stuttgart 1993, S. $3965 f$. 
Europäische Kommission

Europäische Kommission

Fest, Hartmut

Giersch, Herbert

Giersch, Herbert

Hausmann, Hartmut

Hayek, F.A., von

Henseler-Unger, Iris

Heuß, Ernst

Hofman, B. / Koop, M.

Hort, Peter

Irsch, N. / Müller-Kästner, B.

Junkernheinrich, Martin

Junkernheinrich, Martin
„Leitfaden zur Reform der Strukturfonds in der Gemeinschaft", Luxemburg 1989.

„Wettbewerbsfähigkeit und Kohäsion: Tendenzen in den Regionen - Fünfter periodischer Bericht über die sozioökonomische Lage und Entwicklung der Regionen der Gemeinschaft", Luxemburg 1994.

„Vom Knacken industrieller Kerne in Ostdeutschland", in WD, 1993/IX, S. 469 ff.

„Kern und Rand in der spontanen Ordnung“, FAZ vom 21.05.1994.

"Thünen Vorlesung“, Zeitschrift für Wirtschafts- und Sozialwissenschaften, 110(1990).

„Reform der Strukturfonds: Vom Mauerblümchen zum wichtigsten Finanzinstrument", in: Das Parlament vom 30.07.1993

„Die Anmaßung von Wissen“, in: ORDO, Band 26, Stuttgart 1975, S. 13ff.

„Regionale Wirtschaftsförderung in den neuen Bundesländern" in: Jahrbuch für Regionalwissenschaften, 12./13. Jahrgang.

„Wettbewerb“ in: Handwörterbuch der Wirtschaftswissenschaft, Göttingen 1980.

„Die 'Neue Wachstumstheorie' und ihre Bedeutung für die Wirtschaftspolitik“, Kieler Diskussionsbeiträge, 1993.

„Buna, Leuna und die anderen - Beihilfen auf dem Prüfstand“, FAZ vom 07.11.1995.

„Vorschläge zur Weiterentwicklung der regionalen Wirtschaftspolitik", in: Wirtschaftsdienst, 1984, Heft 12, S. 626 ff.

„Eupaenisation of Regional Policy - Supranational impact on German Regional Policy“, IWH Diskussionspapier Nr. 15, Mai 1994.

„Reform des Gemeindefinanzsystems - Zur Position des Sachverständigenrates zur Begutachtung der gesamtwirtschaftlichen Entwick- 
Jürgensen, Harald

Kinkel, Martin

Klemmer / Junkernheinrich

Lichtblau, Karl

Minist. f. Wirtsch. MVP

Minist. f. Wirtsch. MVP

Nolte, D. / Tofaute, $\mathrm{H}$.

North, D.C.

o.V.

o.V.

o.V.

o.V.

o.V. lung“, in: Archiv für Kommunalwissenschaften, II/1992, S. $220 \mathrm{ff}$.

„Raumwirtschaft II: Politik“ in: Handwörterbuch der Wirtschaftswissenschaft, Göttingen 1980 , S. 429-441.

„Abschaffung der Gewerbekapitalsteuer und ihre Kompensation“, WD 1995/V, S. 250 ff.

„Neuordnung des Gemeindefinanzsystems“, Berlin 1994, Band 3 der Reihe „Forum öffentliche Finanzen".

„Privatisierungs- und Sanierungsarbeit der Treuhandanstalt", 1993.

„Jahreswirtschaftsbericht 1995 MecklenburgVorpommern", Schwerin 1995.

„Jahreswirtschaftsbericht Vorpommern 1994“, Schwerin 1994

Mecklenburg„Kahlschlag verhindern - industrielle Kerne erhalten“, WSI Materialien Nr. 36, Düsseldorf 1993.

„Location Theory and Regional Economic Growth“ in: JPE, Bd 23, 1955, S. 243-258.

„Bedeutung weicher Standortfaktoren“, DifuBerichte I/1994.

„Brüssel billigt Förderprogramme“, Handelsblatt vom 09.08.1994.

„EG-Beihilfenkontrolle bezüglich Maßnahmen im Bereich der wirtschaftsnahen Infrastruktur" Papier des Arbeitskreises der EUWirtschaftsreferenten, Herbst 1994

„Ein neuer EU-Fonds für dünnbesiedelte Gebiete - Endspurt bei den Beitrittsverhandlungen“, Die Tageszeitung vom 23.01.1994

„EU-Geld in neuen Ländern schlecht verwaltet", FAZ vom 04.11.1995. 
o.V.

o.V.

o.V.

o.V.

o.V.

o.V.

Olson, $\mathrm{M}$.

Peffekoven, R.

Pennock

Prognos AG

Ridinger, $\mathrm{R}$.

Schäfers, M. / Starbatty J.
„EU-Kommission erhebt Einwände gegen Förderphilosophie der Bundesregierung", Handelsblatt vom 21.03.1994.

„Fördermittel sollen besser kontrolliert werden - Kommissarin Wulf-Mathies reagiert auf Kritik an EU-Struktrufonds", FAZ vom 03.03.1995.

„Geldfluß von Brüssel nach Ostdeutschland stockt", Frankfurter Rundschau vom 31.03.1994.

„Keine Entwicklungspolitik ist die beste für den Mezzogiorno“, FAZ vom 03.01.1997.

„Rebellion im Osten“, Die Zeit vom 13.05.1994

„Streit um Fördermittel beigelegt“", Handelsblatt vom 22.06.1995

„Das Prinzip der fiskalischen Gleichheit: Die Aufteilung der Verantwortung zwischen verschiedenen Regierungsebenen“, in: G. Kirsch, „Föderalismus“, 1977.

„Finanzausgleich I: Wirtschaftstheoretische Grundlagen" in: Handwörterbuch der Wirtschaftswissenschaft, Göttingen 1980.

„Federal and Unitary GovernmentDisharmony and Frustration" in: Behaiviournal Science, IV (April 1959), S. 147-157.

„Instrumente und Informationssysteme für die Wirtschaftspolitik des Landes MecklenburgVorpommern", 1992

„Aktuelle Diskussion zur Finanzausstattung und Reform der EG-Regionalförderung“, in: Wirtschaftsdienst 1992/XII.

"Das Instrumentarium der EG zur Förderung innergemeinschaftlicher Kohäsion", in: Aus Politik und Zeitgeschichte, B28/90, 06.07.1990, S. 3-15 
Scharpf, Fritz W.

Schütterle, P.

Staatskanzlei MVP

Statistisch. Landesamt MVP

Straubhaar, Thomas

Streit, Manfred E.

Thoss, Rainer

trend $\mathrm{GmbH}$

Tullok, G.

Vihanto, Marrtti

Waniek, Roland W.
„Europa nach Maastricht: Markt ohne Demokratie?", in: WD, 1995/II, S. 85 ff.

„EG-Beihilfenkontrolle und kommunale Grundstücksverkäufe“ in: EuZW-Heft 20/1993.

„Die Zukunft des Landes entschlossen gestalten“, Schwerin 1994.

„Statistisches Jahrbuch 1993“, Schwerin 1993.

„Zur optimalen Größe eines integrierten Wirtschaftsraumes: Das Konzept des funktionalen Föderalismus“, Diskussionsbeiträge zur Wirtschaftspolitik, Institut für Wirtschaftspolitik, Universität der Bundeswehr Hamburg, 1993

„Dimensionen des Wettbewerbs - Systemwandel aus ordnungsökonomischer Sicht", in: Zeitschrift für Wirtschaftspolitik, Jg. 44 (1995) Heft 2.

„Ermittlung der konkurrierenden Regionen Interregionale und intraregionale Konkurrenzbeziehungen“, in: „Konkurrenz zwischen kleinen Regionen“" Nomos, 1978, S. 41-62.

„Zum Stand und zur Entwicklung kleiner und mittelständischer Unternehmen des Handwerks, Studie im Auftrag der Handwerkskammer Schwerin, 1994.

„Föderalismus: Zum Problem der optimalen Kollektivgröße" in: G. Kirsch, Föderalismus, 1977.

"Competition Between Local Governments as a Discovery Procedure“, in: Journal of Institutional and Theoretical Economics (JITE), 148(1992).

„EG-Regionalpolitik für die Jahre 1994 bis 1999“, WD 1994/I. 
„Neuordnung der Regionalförderung im Rahmen der Gemeinschaftsaufgabe Verbesserung der regionalen Wirtschaftsstruktur", in: Informationen zur Raumentwicklung, Heft 9/10.1991.

\section{Gesetze / Verordnungen / Programme}

Europäische Kommission

Landesregierung MVP

Minist. f. Wirtsch. MVP

Minist. f. Wirtsch. MVP

Minist. f. Wirtsch. MVP

o.V.

o.V.

o.V.

o.V.
"Gemeinschaftliches Förderkonzept für die Gebiete von Ost-Berlin, MecklenburgVorpommern, Brandenburg, Sachsen-Anhalt, Thüringen und Sachsen 1991-1993“, Brüssel den 13.03.1991.

„Konzeption zur Entwicklung ländlicher Räume in Mecklenburg-Vorpommern", Schwerin 1992.

„Der Mittelstand in MecklenburgVorpommern“, Schwerin, 1990.

„Operationelles Programm des Europäischen Fonds für regionale Entwicklung für das Land Mecklenburg-Vorpommern im Zeitraum 19911993“" vom 08.03.1991.

„Tourismuskonzeption

MecklenburgVorpommern“, Schwerin 1993.

„Erstes Landesraumordnungsprogramm Mecklenburg-Vorpommern", der Wirtschaftsminister, Schwerin 1993.

Dreiundzwanzigster Rahmenplan der Gemeinschaftsaufgabe „Verbesserung der regionalen Wirtschaftsstruktur", vom 28.04.1994, Drucksache 12/7175.

Einundzwanzigster Rahmenplan der Gemeinschaftsaufgabe „Verbesserung der regionalen Wirtschaftsstruktur", vom 13.05.1992, Deutscher Bundestag, Drucksache 12/2599.

Gesetz über die Gemeinschaftsaufgabe „Verbesserung der regionalen Wirtschaftsstruktur" vom 06.10.1969, BGBI.I, S. 1861. 
o.V.

o.V.

o.V.

o.V.
Verordnung (EG) Nr. 2081/93, Abl (EG) L 193 vom 31.07.1993.

Verordnung (EWG) Nr. 2052/88 des Rates über Aufgaben und Effizienz der Strukturfonds und über die Koordinierung ihrer Interventionen untereinander sowie mit denen der Europäischen Entwicklungsbank und anderen vorhandenen Finanzierungsinstrumenten. Amtsbl. EG, Nr. L 158/9 vom 15.07.1988.

Verordnung (EWG) Nr. 3575/90 des Rates vom 04. 12.1990 über die Intervention der Strukturfonds im Gebiet der ehemaligen Deutschen Demokratischen Republik.

Zweiundzwanzigster Rahmenplan der Gemeinschaftsaufgabe „Verbesserung der regionalen Wirtschaftsstruktur", vom 19.05.1993, Drucksache 12/4850. 


\section{SCHRIFTEN ZUR WIRTSCHAFTSTHEORIE UND WIRTSCHAFTSPOLITIK}

\section{Herausgegeben von Rolf Hasse, Wolf Schäfer,}

Thomas Straubhaar, Klaus W. Zimmermann

Band 1 Lars Bünning: Die Konvergenzkriterien des Maastricht-Vertrages unter besonderer Berücksichtigung ihrer Konsistenz. 1997.

Band 2 Andreas Henning: Beveridge-Kurve, Lohnsetzung und Langzeitarbeitslosigkeit. Eine theoretische Untersuchung unter Berücksichtigung des Insider-Outsider-Ansatzes und der Entwertung des Humankapitals. 1997.

Band 3 Iris Henning: Die Reputation einer Zentralbank. Eine theoretische Untersuchung unter besonderer Berücksichtigung der Europäischen Zentralbank. 1997.

Band 4 Rüdiger Hermann: Ein gemeinsamer Markt für Elektrizität in Europa. Optionen einer Wettbewerbsordnung zwischen Anspruch und Wirklichkeit. 1997.

Band 5 Alexander Tiedtke: Japan und der Vorwurf des Trittbrettfahrenverhaltens in der US-amerikanisch-japanischen Allianz. 1997.

Band 6 Wolfgang Grimme: Ordnungspolitisches Konzept der Regionalpolitik. Darstellung der Defizite und des Reformbedarfs der Regionalpolitik am Beispiel Mecklenburg-Vorpommerns. 1997. 


\section{Die doppelte deutsche Diktaturerfahrung}

\section{Drittes Reich und DDR - ein historisch-politikwissenschaftlicher Vergleich}

Frankfurt/M., Berlin, Bern, New York, Paris, Wien, 2., neubearb. Aufl. 1996. $330 \mathrm{~S}$.

ISBN 3-631-30393-9 • br. DM 95.-*

Zwei Diktaturen auf deutschem Boden haben in der Geschichte des 20. Jahrhunderts entscheidende Folgen gehabt. So sehr das Dritte Reich und die DDR am entgegengesetzten Spektrum des politischen Extremismus angesiedelt waren, stellt sich doch die Frage, inwieweit die beiden Diktaturen strukturelle und habituelle Analogien aufgewiesen haben. Freiburger Studierende der Politikwissenschaft haben die jeweiligen Prozesse der Machtergreifung, die Mechanismen der Machtsicherung und die Umstände des Machtverfalls ebenso im Vergleich untersucht wie die Frage nach der Funktion der beiden entgegengesetzten Ideologien oder die Rolle der Wirtschaftspolitik in beiden Systemen. Ihre Analysen sind ein Beitrag zu der erst begonnenen wissenschaftlichen Auseinandersetzung über Gemeinsamkeiten und Unterschiede zwischen dem Dritten Reich und der DDR.

„Das Unterfangen kann in der Tat insgesamt als gelungen bezeichnet, die Fortsetzung empfohlen werden. Die Aufsätze sind gut strukturiert, logisch aufgebaut, informativ gehalten, auf dem neuesten Forschungsstand, und der common sense ist unübersehbar".

(Prof. Eckhard Jesse, Neue Zürcher Zeitung, in einer Rezension zur ersten Auflage)

\footnotetext{
Frankfurt/M - Berlin - Bern - New York · Paris - Wien

Auslieferung: Verlag Peter Lang AG

Jupiterstr. 15, CH-3000 Bern 15

Telefax (004131) 9402131

«inklusive Mehrwertsteuer

Preisänderungen vorbehalten
} 